\title{
Cosmic Evolution Physics (I) for Supergravity and Dark-Energy with Super-Inflation
}

\author{
Li Zongcheng \\ ( Research Lab of Interdisciplinary Science, Soochow University, Soochow, P. R. CHINA )
}

To solve a series of major problems, such as the singularity before the Big Bang, the inflation of the early universe and the accelerating expansion of the present universe, this series of studies start with super gravity, dark energy and dark matter, go beyond the surface of a lot of observation data, and systematically reveal the arche-internal mechanism of all things, to establish the arche-unity of physics, and give a natural and reasonable explanation. At present, there are at least three basic pathways that are leading our research field to repulsion against gravitation, quantum repulsion against quantum gravitation and high-dimensional superrepulsion against high-dimensional supergravity, that is, the research on the accelerating expansion mechanism of the current universe, the research on the dynamic mechanism of the early cosmic inflation, and the research on the rebound mechanism near the singularity before the Big Bang. In this series of studies, the arche problem of everything is always placed under the extreme environmental conditions of the initial universe, the starting point of scientific logic is closely combined with the starting point of the universe history, the pseudo vacuum energy considered by the inflationary universe model, the Higgs field predicted by the standard physical model and the dark energy speculated by the observational cosmology are reduced to the positive energy system with strong negative pressure, and they are reduced to the repulsion (all the resistance to gravity) that forms a conjugate relation with gravity. In our opinion, dark energy and dark matter are essentially at the level of quantum gravity and quantum repulsion. Therefore, we not only consider the conventional quantum effect and non commutative quantum effect, but also further consider the arche-conjugate effect and the cluster resonance-at-cofrenquence effect. For noncommutative space-time, we put forth the ultimate supersymmetry, the arche-conjugation and the cluster resonance-at-cofrenquence. A relatively complete natural mechanism forms such an important inference: the closer they get to Planck scale, the stronger the conventional quantum effect, non commutative quantum effect, arche-conjugate effect and co-frequency resonance effect are. In the high-dimensional space-time (the 11 dimensional hyperspace compactly reduced to $M_{4} \times N_{r}$ ), corresponding to the introduction of supergravity with the supersymmetry between the graviton and the gravitino, we put forth the superrepulsion with the supersymmetry between the repulsion and the repulsitino, and establish the arche-conjugate relationship between the high-dimensional supergravity and the high-dimensional superrepulsion. Therefrom, we propose a basic inference that can be promoted to the principle of hyperholography of high-dimensional universe (or multiuniverse): in a parallel universe formed by mutual coupling and holographic correspondence between the $P$ universe dominated by negative pressure (high-dimensional superrepulsion and quantum repulsion of accumulating positive energy, mainly composed of dark energy) and the $N$ universe dominated by positive pressure (high-dimensional supergravity and quantum gravity of accumulating negative energy, mainly composed of dark matter), there is a correspondence between the small-scale physics (ultraviolet cutoff) of the $P$ universe and the large-scale physics (infrared cutoff) of the $N$ universe, while there is a correspondence between the large-scale physics (ultraviolet cutoff) of the $P$ universe and the small-scale physics (infrared cutoff) of the $N$ universe. In the new research paradigms set in this series, the arche-conjugation between the high-dimensional supergravity and 
the high-dimensional super repulsion, as well as between the quantum gravity and the quantum repulsion, is higher than the supersymmetry, so it becomes the core concept of the new theory. The arhce-unity of physics is a super unity that transcends the grand unification of four basic interaction, which is formed between high-dimensional supergravity (related to dark matter) and high-dimensional superrepulsion (related to dark energy), between quantum gravity (related to dark matter) and quantum repulsion (related to dark energy). From the basic component point of view, before or after the big bang, the gravitons and repulsons are coupled together, the negative energy contraction dominated by the positive pressure and the positive energy expansion dominated by the negative pressure work alternately, forming the arche-conjugate pulsaton of neither the point particle nor the linear superstring. Under the big unified framework of quantum gravitational field and quantum repulsive field, this series of work comprehensively expands the general relativity and loop quantum ring gravitation theory, sets up the quantum repulsive field equation corresponding to the quantum gravitational field equation, and then establishes the quantum hedge-unified field equation describing the interaction of quantum gravitational field and quantum repulsive field; On this basis, the quantum repulsive universe equation corresponding to the quantum gravitational universe equation is established, thus a set of new equations describing the expansive universe with quantum hedge-unified field are established. On the one hand, scalar disturbance is considered as the exhibition of repulsive effect, on the other hand, tensor disturbance is considered as the exhibition of gravitational effect. In the initial stage of the big ripping, due to the mutual restraint of quantum repulsion and quantum gravity, the scalar and tensor perturbations are not obvious. However, at the end of the big ripping, the scalar and tensor perturbations are very significant, and there is a large power spectral exponential run. Between particle physics and cosmology, we improve and expand the path integral of quantum gravity, create the path integral of quantum hedge-unified field, and set up the basic equation of quantum cosmic-synergetic dynamics, and then establish the basic equation of quantum-cosmic paradigm dynamics. Thus we put up the operator distribution function and its dynamic equation of the quantum arche- pulsaton, and finally use the Wigner-Ville distribution as a non-linear time-frequency distribution to establish the Wigner joint-distribution of quantum-conjuagted pulsation function, so as to analyze the arche of all things with three evolutionary forms: quantum chaotic-pulsaton, quantum quasi-spherical pulsaton and quantum spherical-pulsaton. In this series of work, the mechanism to avoid the singularity of the universe is put forward fundamentally, and the dark energy and dark matter are explained through new mechanisms and effects at the level of quantum gravity. Between particle physics and cosmology, we improve and expand the path integral of quantum gravity, establish the path integral of quantum hedge-unified field, thus establish the basic equation of quantum cosmic-synergy dynamics, and then establish the basic equation of quantum cosmic-paradigm dynamics. Thus the covariant gravitational mass and the covariant inertial mass formed by the ultra-synergy between particles and the universe are presented, and the equivalence hypothesis on the covariant non-inertial system which can be elevated to the basic principle, is presented. Based on this hypothesis, a systematic calculation and analysis of binary stars composed of neutron stars and black holes are made. The master equation, variance and Fokker-Planck equation for the birth-death and migration of a large number of particles are established between binary stars composed of neutron stars and black holes.

Keyword: cosmic singularity; super repulsion; arche-conjugation; holographic unification; arche-pulse dynamics

PACS: $02.30 . \mathrm{Tb}$; 05.45.Mt; 11.25.Yb; 12.10.Dm; 98.80.Cq; 98.80.Qc

\section{Introduction}

In the past two or three decades, various kinds of cosmological observations have developed rapidly. Cosmological observation experiments ${ }^{[1]-[5]}$, such as cosmic background detector (COBE), Sloan Digital Sky Survey Satellite (SDSS), Wilkinson Microwave Anisotropy Probe (WMAP), type Ia 
supernova project, Planck satellite, etc, have brought us into exciting age of precision cosmology. WMAP data show that ${ }^{[6]-[8]}$, our universe is almost flat in space, i.e. $\Omega_{-}$(total) $=1.02 \pm 0.02$. The study of large-scale structure distribution of galaxy clusters shows the existence of dark matter, and its proportion is $\Omega_{-}(\mathrm{CDM})=0.27 \pm 0.04$. All these results strongly show that there is an extra material component with strong negative pressure, which accounts for about $2 / 3\left(\Omega_{-}\right.$(de) $\left.=0.67 \pm 0.06\right)$ of the total. It is evenly distributed in the whole space and accelerates the expansion of the universe. Such a material component is called dark energy, which contradicts the thermal big bang cosmological model based on common baryonic matter. According to the cosmological model of the big bang, the universe should slow down and expand. In order to explain the current accelerating expansion of the universe in the framework of the thermal big bang cosmological model, it needs to be modified. So far, many models have appeared, such as cosmological constant $\Lambda$, slow-rolling scalar field, quintessence, phantom and Quintom, etc. ${ }^{[9]-[14]}$.

In order to unify quantum theory and general relativity, researchers have opened up some new ways to establish the theory of quantum gravity for more than half a century, including string theory, membrane theory, loop quantum gravity theory, holographic principle and $\mathrm{M}$ theory (or mystery theory), inverse de Sitter space (AdS) / conformal field theory (CFT), etc ${ }^{[15]-[18]}$. However, when people think they know everything about nature, it always hides a greater surprise ${ }^{[19]-[21]}$. From quantum mechanics, quantum field theory and gravitation theory to quantum gravity theories such as superstring theory, $\mathrm{M}$ theory, AdS/CFT and holographic duality theory, etc, in modern physics there have been the differences, controversies and paradoxes between objective reality and subjective perception. As far as the present situation is concerned, quantum mechanics is not self-consistent. It has been in the entanglement of traditional interpretation, PTV ensemble interpretation and statistical interpretation. It still suffers from the Einstein-Rosen-Podolsky paradox ${ }^{[22]-[24]}$.

At present, a series of major knotty problems such as the singularity of the universe before the big bang, the inflation of the early universe, and the accelerating expansion of the current universe are still on display in front of us ${ }^{[25]-[30]}$, which involve super gravity, dark energy, dark matter and other issues, and ultimately involve the arche of all things and the noumenon of the world. It is not clear how the universe can avoid the singularity brought about by the great collapse before it is created, what is the reason for the emergence of noncommutative quantum relations on the Planck scale? Both cosmological and particle physics researchers have lacked an in-depth systematic research on the interaction between the expansive force caused by the Higgs field and all the internal gravitational forces, as well as the interaction between the expansive force caused by the Higgs field and all the internal strong forces. In order to explain theoretically the problem of eliminating or avoiding the singularity of the universe, there have been various researches, among which some works have proposed theories and methods such as large rebound mechanism, quantum effect, mathematical calculation and processing (for example, semiclassical loop quantum universe model and membrane world model $)^{[31]-[34]}$, so as to eliminate or avoid the singularity of the universe. However, these analysis, interpretation and calculation need to be further fundamentally solved. To a certain extent, we should avoid some magic, mystery and speculation. The great difficulty ${ }^{[35]-[37]}$ brought by the discovery of dark energy is that why is its energy density so small? Because if dark energy is a cosmological constant, the energy density of the given dark energy is about $6 \times 10^{-9} \mathrm{erg} \cdot \mathrm{cm}^{-3}\left(\right.$ or $\rho_{A} \approx 10^{-47} \mathrm{GeV}^{4}$ ). However, according to quantum field theory, the micro origin of the cosmological constant should be vacuum 
energy, and the energy density of the estimated dark energy is about $10^{115} \mathrm{erg} \cdot \mathrm{cm}^{-3}$ (or $\rho_{\text {vac }} \approx 10^{74}$ $\mathrm{GeV}^{4}$, even if considering Considering the supersymmetry effect, the contribution of Boson and fermion to the vacuum energy is cancelled, however, because the supersymmetry has not been detected in the energy scale of $\mathrm{TeV}$, the estimated vacuum energy is still far greater than the observation result), which means that the theoretical calculation result is about 120 orders of magnitude higher than the experimental observation result! But, if the energy density of dark energy remains at $10^{115} \mathrm{erg} \cdot \mathrm{cm}^{-3}$, the huge repulsion generated by dark energy will accelerate the expansion of the universe extremely rapidly, so that stars, galaxies and the current large-scale structure of the universe cannot be formed under the influence of gravity.

The nature of dark energy is still unclear to people ${ }^{[28]-[30]}$. First of all, people don't know exactly what dark energy is. Is it a cosmological constant or a physical field with dynamic mechanism? How does it affect the fate of the universe? Secondly, it is not clear whether there is a direct interaction between dark energy and dark matter. If this interaction exists, how does it affect the evolution of the universe? Third, it is not known for sure whether Einstein's general relativity is correct on the scale of the whole universe. In principle, the universe expansion history consistent with the existence of dark energy can be obtained by modifying the gravity at the cosmic scale. So, is the accelerated expansion of the universe caused by the existence of dark energy or by modifying the gravity?

In explaining the accelerated expansion of the universe, there have been various models of dark energy and dark matter ${ }^{[9]-[12][38]-[49]}$, including the CDM model, wCDM model, CPL model, slow-rolling scalar field model, quintessence model, phantom model, Quintom model, Chaplygin gas model, constant $w$ model, $\alpha$ dark energy model, cosmological constant model, and generalized Chaplygin gas model as a unified model of dark energy and dark matter, holographic dark energy model, holographic gas model, etc. These models can be roughly classified as symmetry model, human selection principle, adjustment mechanism, modified gravity theory, quantum gravity, holographic principle, reaction mechanism and phenomenological model, etc. In M Theory and IIA string theory, the anthropic principle can be realized, i.e. in the model of the so-called string landscape that first proposed by L. Suskind, string theory has many vacuums ${ }^{[48][49]}$. However, they also fail to explain at the basic physical level the origin of dark matter, dark energy and primitive perturbation with near scale invariant energy spectrum: in this sense, they are only some useful parametric forms. Researchers in related fields estimate that these simple assumptions may not be completely accurate after they have a better understanding of related basic physics. For example, inflation theory predicts that the space curvature of the universe is in the order of $10^{-5}$ to $10^{-4}$. In addition, it's hard to believe that the temperature of dark matter is absolute zero.

In the process of establishing the unified theory of interaction, between quantum mechanics and general relativity, the quantization of gravitational field is an important difficult problem, the continuous background space and time is the root of ultraviolet divergences in quantum field theory. Under extreme conditions, the spatiotemporal singularity is induced in general relativity, and the non -renormalization appears, to have shown that the theory itself is imperfect. Therefore, quantization and general relativity are mutually exclusive $\mathrm{e}^{[50]-[52]}$. The two theories should be unified in a larger theoretical framework. In the past few decades, there have been many attempts to overcome this basic difficult, such as super symmetric gravitational theory, string theory and loop quantum gravity theory, M theory, as well as AdS / CFT and the dual holographic principle, etc. have successively tried to 
become the candidate object of this more theoretical framework ${ }^{[53]-[55]}$. However, there are only two attempts approved in some degree, one is the loop quantum gravity theory with non perturbation, the other is string theory. Loop quantum gravity is reduced to quantum geometry ${ }^{[56]-[57]}$. R. Penrose's algebraic form of the discrete space model is similar to the algebraic relation of the spin, which is called the spin network. The expressions introduced by A., Ashtekar and others in the scheme of canonical quantization are based on the self-dual spin connection as the fundamental field quantity ${ }^{[58]}$. T., Jacobson, and L. Smolin found that the Wilson loop of the Ashtekar variable satisfies the Wheeler-DeWitt equation. On this basis, C. Rovelli and L. Smolin proposed the Wilson loop as the basic state of quantum gravity, thus forming an important scheme of modern quantum gravity theory ${ }^{[59]-[61]}$, where the use of metric fields is avoided, and the so-called "background gauge" is no longer introduced. This background independence of Loop quantum gravity is regarded to conform the physical nature of quantum gravity, because general relativity requires space-time itself to be determined by the law of dynamics, quantum gravity theory is the theory about space-time itself.

At present, there are at least three places where quantum mechanics and general relativity are still in a tug-of-war. First, general relativity predicts that it will fail at the singularity, while quantum mechanics will be incompatible with general relativity near the singularity. Secondly, since the position and velocity of a particle cannot be determined simultaneously under the Heisenberg uncertainty principle of quantum mechanics, it is not clear how to determine the gravitational field of a particle. Finally, the contradiction between the two theories is not only a logical contradiction, but also a dilemma between the "violation of Bell inequality caused by quantum mechanics" (implying the influence of superluminal speed) and "the speed of light as the limit of speed in relativity". The solution to the first two points may come from a better understanding of general relativity.

Semiclassical loop quantum cosmology shows us the evolution of the universe from contraction to rebound and then to expansion. In addition, some studies show that ${ }^{[62]-[64]}$, the intervention of loop quantum effect makes it possible for the early expansion of the universe to enter a super inflation stage. At this stage, the expansion of the universe is very slow, but the transformation of Hubble parameters is very fast, so it can also solve the problem of horizon and flatness, and the disturbance of its original scalar field can produce scale invariant spectrum.

In fact, string theory, membrane theory, loop quantum gravity theory, super gravity holography principle and $\mathrm{M}$ theory, which are the theoretical basis of cosmology, are basically established by simplification under the condition of breaking away from the extreme background of the universe, falling into the strange circle of mathematical games, lacking in in-depth discussion of the essence of the physical world. Up to now, people have not found a convincing realistic model of high energy physics theory, which should include the inflation field needed by the inflation theory. This reflects people's ignorance of a self consistent theoretical framework of high energy physics. Due to the lack of new ideas, the construction of the new theoretical model is difficult, so it can only use the new astronomical observation to do further promotion. Cosmology with a congenital deficiency can not provide strong support for particle physics to further advance to a wider field and deeper level. Therefore, for the universe in the formation of basic particles and the basic particles in the evolution of the universe, both the reduction under the principle of simplicity and the synthesis under the principle of complexity are not enough to establish a reasonable theoretical basis. The best way of research should and can only be to form a summary of relevant research on particle physics and cosmology 
under the principle of fusion.

Modern physics and cosmology are facing a series of major knotty problems of major ${ }^{[65]-[68]}$ : from the dimension and nature of space-time to the spontaneous rupture of super-symmetry, from quantum de-coherence mechanism to the unification of the four fundamental forces, from the origin of black hole entropy to the exploring of the superstring theory for explore the self-consistent formula of action quantities, from macroscopically-irreversibility in statistical mechanics to the limitation of the historical summation method in physics, a series of enormous challenges in forming provide the objectives and tasks for the third upcoming scientific revolution.

Researchers can compare the latest data from astrophysics and particle physics experiments with precise and quantitative predictions made by specific physical models. However, it is important to note that this is different from the higher goal: to develop a theoretical framework that can deduce a specific model of the observable universe without the aid of experimental data. Like any scientific theory, the inflation is not needed to solve all possible problems. Existing inflation models, like all scientific theories, are based on a set of assumptions, and in order to understand these assumptions, we may need some deeper theories.

In our opinion, from Newton's gravity to Einstein's gravitational field, from strong and electro -weak force to quantum gravity and high-dimensional super gravity, classical physics and modern physics are basically deduced in the generalized gravity category. However, a series of major knotty problems, such as the singularity before the Big Bang, the inflation of the early universe and the accelerating expansion of the present universe, have gone far beyond the scope of generalized gravity, while dark energy and dark matter are essentially at the level of quantum gravity and quantum repulsion.

At present, there are at least three basic pathways that are leading our research field to repulsion against gravitation, quantum repulsion against quantum gravitation and high-dimensional superrepulsion against high-dimensional supergravity, that is, the research on the accelerating expansion mechanism of the current universe, the research on the dynamic mechanism of the early cosmic inflation, and the research on the rebound mechanism near the singularity before the Big Bang. Therefore, it is necessary to go beyond the existing models and calculation methods, beyond the power spectrum, spectral index, tensor-scalar ratio and other data of the inflation, set a broader and stronger framework, and establish a more natural and profound theory, so as to explore the essential factors behind the cosmic inflation, find more information about the early universe, and reveal more physical laws on a smaller scale. In light of some major basic difficult problems in particle physics, astrophysics and cosmic physics (cosmic singularity caused by big collapse, inflation of early universe, accelerating expansion of current universe and related dark energy, dark matter, etc.), the physical research about the origin of all things is placed in the extreme environmental conditions of the initial universe at the starting point of scientific logic, which should be as deep as possible to systematically reveal the noumenon behind the physical phenomenon.

The main task of this series of research is to establish a perfect theoretical framework for calculating the interaction between high-dimensional supergravity and dark energy (with strong negative pressure), describing the history of cosmic inflation-expansion and structural growth, so that it can be applied to any dark energy theoretical model and any interaction model.

The physical ecosystem of interaction between quantum gravity and quantum repulsion proposed 
and studied in this paper is an accumulation system of all things centered on the source of quantum unification, which involves not only the scope of quantum system, but also the scope of macro system; it involves not only the material aspects or physical matters, but also the complex things composed of many elements. Its existence and evolution range is generally a little larger than the horizon. In the quantum physical ecosphere, the source of quantum unifying force is not unique, and may have multiple source of quantum unifying force.

For the cosmic ecosphere of the multiverse world, this series of work attempt to set up a larger theoretical framework at a deeper level, and combine the creation of the universe with the unification of the fundamental physic functions at the starting point of the universe, so as to study. On the one hand, the arche-unification of the fundamental physic functions is re-studied under the extreme conditions of the creation of the universe. On the other hand, the creation of the universe is re-studied on the basis of the arche-unification of the fundamental physical functions.

Different from the models of superstring, membrane and loop quantum, which are separated from the extreme environmental conditions of the initial universe and fall into the abstract high-level mathematical game since they were born, this series of researches always put the arche problem of all things under the extreme environmental conditions of the initial universe, closely combine the starting point of scientific logic with the starting point of the history of the universe, so that the pseudo vacuum energy of the inflationary universe model, Higgs field predicted by standard physical model and dark energy inferred by observation cosmology are all attributed to positive energy system with strong negative pressure, and they are also attributed to the repulsion (all factors of resistance to gravity) which forms a conjugate relationship with gravity. In our opinion, dark energy and dark matter are essentially at the level of quantum gravity and quantum repulsion. Therefore, we not only consider the conventional quantum effect and non commutative quantum effect, but also further consider the arche-conjugate effect and the cluster resonance-at-cofrenquence effect. For noncommutative space-time, we put forth the ultimate supersymmetry, the arche-conjugation and the cluster resonance-at-cofrenquence. All kinds of strange phenomena converge into one possibility, that is, the closer they get to Planck scale, the stronger the conventional quantum effect, non commutative quantum effect, arche-conjugate effect and cluster resonance-at-cofrenquence effect are. In the high-dimensional space-time (the 11 dimensional hyperspace compactly reduced to $M_{4} \times N_{r}$ ), corresponding to the introduction of supergravity with the supersymmetry between the graviton and the gravitino, we put forth the superrepulsion with the supersymmetry between the repulsion and the repulsitino, and establish the arche-conjugate relationship between the high-dimensional supergravity and the high-dimensional superrepulsion.

Therefrom, we propose a basic inference that can be promoted to the principle of hyperholography of high-dimensional universe (or multiuniverse): in a parallel universe formed by mutual coupling and holographic correspondence between the $P$ universe dominated by negative pressure (high-dimensional superrepulsion and quantum repulsion of accumulating positive energy, mainly composed of dark energy) and the $N$ universe dominated by positive pressure (high-dimensional supergravity and quantum gravity of accumulating negative energy, mainly composed of dark matter), there is a correspondence between the small-scale physics (ultraviolet cutoff) of the $P$ universe and the large-scale physics (infrared cutoff) of the $N$ universe, while there is a correspondence between the large-scale physics (ultraviolet cutoff) of the $P$ universe and the small-scale physics (infrared cutoff) of 
the $N$ universe.

In the new research paradigms set in this series, the arche-conjugation between the highdimensional supergravity and the high-dimensional super repulsion, as well as between the quantum gravity and the quantum repulsion, is higher than the supersymmetry, so it becomes the core concept of the new theory. The arhce-unity of physics is a super unity that transcends the grand unification of four basic interaction, which is formed between high-dimensional supergravity (related to dark matter) and high-dimensional superrepulsion (related to dark energy), between quantum gravity (related to dark matter) and quantum repulsion (related to dark energy).

From the perspective of the whole universe, before the big bang or in the compact 5-11 dimensional space-time, the high-dimensional supergravity field (collapse field) and the gravitational field (contracted field) are dominant; after the big bang or in the extended 4-dimensional space-time, the high-dimensional superrepulsive field (inflation field) and the repulsive field (expansive field) are dominant. From the basic component point of view, before or after the big bang, the gravitons and repulsons are coupled together, the negative energy contraction dominated by the positive pressure and the positive energy expansion dominated by the negative pressure work alternately, forming the arche-conjugate pulsaton, and then forming the quantum conjugate pulsaton.

Under the big unified framework of quantum gravitational field and quantum repulsive field, this series of work comprehensively expands the general relativity and loop quantum ring gravitation theory, establishes the quantum repulsive field equation corresponding to the quantum gravitational field equation, and then establishes the quantum hedge-unified field equation describing the interaction of quantum gravitational field and quantum repulsive field; On this basis, the quantum repulsive universe equation corresponding to the quantum gravitational universe equation is established, thus a set of new equations describing the expansive universe model under the condition of fully considering the hedge effect of quantum gravitational field and quantum repulsion field, which can be called the expansive universe equation of quantum hedge-unified field theory, is established. On the one hand, scalar disturbance is considered as the reflection of repulsive effect, on the other hand, tensor disturbance is considered as the reflection of gravitational effect. In the initial stage of the big ripping, due to the mutual restraint of quantum repulsion and quantum gravity, the scalar and tensor perturbations are not obvious. However, at the end of the big ripping, the scalar and tensor perturbations are very significant, and there is a large power spectral exponential run.

Between particle physics and cosmology, we improve and expand the path integral of quantum gravity, create the path integral of quantum hedge-unified field, and set up the basic equation of quantum cosmic-synergetic dynamics, and then establish the basic equation of quantum-cosmic paradigm dynamics. Thus we put up the operator distribution function and its dynamic equation of the quantum arche-pulsaton, and finally use the Wigner-Ville distribution as a non-linear time-frequency distribution to establish the Wigner joint-distribution of quantum-conjugated pulsation function, so as to analyze the arche of all things with three evolutionary forms: quantum chaotic-pulsaton, quantum quasi-spherical pulsaton and quantum spherical-pulsaton. Between particle physics and cosmology, we improve and expand the path integral of quantum gravity, establish the path integral of quantum hedge-unified field, thus establish the basic equation of quantum cosmic-synergy dynamics, and then establish the basic equation of quantum cosmic-paradigm dynamics. In this paper, the covariant gravitational mass and the covariant inertial mass formed by the ultra-synergy between particles and the 
universe are presented, and the equivalence hypothesis on the covariant non-inertial system which can be elevated to the basic principle, is presented. Based on this hypothesis, a systematic calculation and analysis of binary stars composed of neutron stars and black holes are made. The master equation, variance and Fokker-Planck equation for the birth-death and migration of a large number of particles are established between binary stars composed of neutron stars and black holes.

In this series of work, the mechanism to avoid the singularity of the universe is put forward fundamentally, and the dark energy and dark matter are explained through new mechanisms and effects at the level of quantum gravity. Compared with the unity of strong force, electro-weak force and gravity, the unity of quantum gravitation and quantum repulsion, or the unity of high-dimensional supergravity and high-dimensional superrepulsion, is a more basic unity with ultimate arche-meaning.

\section{Necessity of Repulsive Force in Elimination of Singularity}

At present, there are at least three basic pathways that are leading our research field to repulsion against gravitation, quantum repulsion against quantum gravitation and high-dimensional superrepulsion against high-dimensional supergravity, that is, the research on the accelerating expansion mechanism of the current universe, the research on the dynamic mechanism of the early cosmic inflation, and the research on the rebound mechanism near the singularity before the Big Bang.

It has been seen for a long time that although the inflation model has achieved great success, as a model under the framework of classical gravitational field theory, it is impossible to give a solution to the singularity problem of the big bang. The general tendency is to think that the solution of the singularity problem of the universe needs a theory of quantum gravity. Therefore, in order to solve the difficulties in the inflation model, there are some works to find semi quantum solutions, such as the rebound universe, cyclic universe and other models obtained by introducing semi classical loop quantum theory or string theory ${ }^{[62]-[64]}$. In these models, the big bang singularity is replaced by a smooth rebound, so the singularity problem can be solved to some extent.

To find a fundamental way to solve the singularity problem of the universe, we start with a brief analysis of the relevant quantum effects and the big rebound. For the big rebound at the end of the big collapse of the universe, the repulsion and high-dimensional superrepulsion that we systematically introduce should be the basic motivation.

Let's do a simple analysis first: We have noticed that in Einstein's gravitational field equation, the physical meaning of the universe constant $\Lambda$ is the strong negative pressure field which plays the role of repulsion. If we rewrite the formula $(1 b)$ in Friedmann equation

$$
\begin{gathered}
H^{2}=\frac{\dot{a}^{2}}{a^{2}}=\frac{8 \pi G}{3} \rho-\frac{k}{a^{2}}+\frac{\Lambda}{3} \\
\frac{\ddot{a}}{a}=-\frac{4 \pi G}{3}(\rho+3 p)+\frac{\Lambda}{3}
\end{gathered}
$$

as follows: 


$$
F=-\frac{G M}{R^{2}}+\frac{\Lambda}{3} R, \quad R=a
$$

It can be seen that the repulsion force produced by cosmological constant increases with the increase of distance. Whether the expansion of the universe slows down or accelerates depends on the contrast between the two terms on the right of equation (2), that is, the contest between the gravity of matter and the repulsion of cosmological constant. In the distant past, cold dark matter dominated the universe, slowing down the expansion of the universe; about 5 billion years ago, cosmological constants began to dominate gradually, and the expansion of the universe began to accelerate.

According to the semiclassical theory of loop quantum cosmology, it is considered that ${ }^{[62]-[64]}$, when the particle energy approaches the Planck energy scale, a large rebound will appear, which can avoid the singularity from the big bang. Since the loop quantum universe takes the regular form, we use Hamiltonian language to discuss it. For the classical straight FRW universe (Friedmann-Robertson -Walker), its Hamiltonian constraint can be simply expressed as

$$
H_{c l}=-\frac{3}{8 \pi G \gamma^{2}} \sqrt{p} c^{2}+H_{M}
$$

here $p=a^{2}, c=\gamma \dot{a}, \gamma \approx 0.2375, H_{M}$ is the Hamiltonian of the material part. Define energy density:

$$
\pi \equiv \frac{E}{V}=\frac{H_{M}}{a^{3}}
$$

If $H_{M} \approx 0$, the Friedmann equation for $k=0$ (which corresponds to a flat universe in FRW scale) can be obtained :

$$
\dot{H}=-4 \pi G(p+\rho)+\frac{k}{a^{2}}
$$

In this equation, when $a \rightarrow 0$, the energy density is divergent, which is the singularity time of the big bang. When the loop quantum gravity effect cannot be ignored, the original Hamiltonian constraint is modified as follows:

$$
H_{L Q C}=-\frac{3}{8 \pi G \gamma^{2} \bar{\mu}^{2}} \sqrt{p} \sin ^{2}(\bar{\mu} c)+H_{M}
$$

where $\bar{\mu}$ is the length of the circle that makes up the smallest facet. When $\bar{\mu} \rightarrow 0$, there is $\sin ^{2}(\bar{\mu} c) / \bar{\mu}^{2} \rightarrow 1, \quad H_{L Q C} \rightarrow H_{c l}$ will return to the classical limit. Through Hamiltonian equation:

$$
\dot{p}=\left\{p, H_{L Q C}\right\}=-\frac{8 \pi G \gamma}{3} \frac{\partial H_{L Q C}}{\partial c}=\frac{2 a}{\gamma \bar{\mu}} \sin (\bar{\mu} c) \cos (\bar{\mu} c)
$$

By substituting $p=a^{2}$, the evolution of scale factor with time can be obtained:

$$
\dot{a}=\frac{1}{\gamma \bar{\mu}} \sin (\bar{\mu} c) \cos (\bar{\mu} c)
$$

On the other hand, from the Hamiltonian constraint $H_{L Q C} \approx 0$, we can get: 


$$
\sin ^{2}(\bar{\mu} c)=\frac{8 \pi G \gamma^{2} \bar{\mu}^{2}}{3 a} H_{M}
$$

By combining the above two equations, the modified Friedmann equation is obtained:

$$
H^{2}=\frac{8 \pi G}{3} \rho\left(1-\frac{\rho}{\rho_{c}}\right)
$$

where the critical density is

$$
\rho_{c}=\frac{\sqrt{3}}{16 \pi^{2} \gamma^{3}} \rho_{p}, \quad \rho_{p}=G^{-2} \text { is Planck density. }
$$

The appearance of the critical density $\rho_{c}$ is a quantum effect, which makes the energy density $\rho$ of the universe no longer diverge in any case, so the curvature of space-time is no longer diverging. When the quantum effect is negligible, that is $\hbar \rightarrow \infty$, then $\rho_{c} \rightarrow \infty$, this goes back to the classical case. In particular, in the very early universe, $\rho=\rho_{c}$ was a rebound point, which replaced the singularity from big bang in the classical theory.

Therefore, semiclassical loop quantum cosmology shows us the evolution of the universe from contraction to rebound and then to expansion.

The membrane world model is a theory of gravitation ${ }^{[69]-[72]}$ based on the string theory of highdimensional space-time. In the image of the membrane world, all matter in the universe, including light, can only be confined to the four-dimensional hypersurface of this high-dimensional space-time, which is called a membrane. However, gravity can exist in other invisible extra dimensions, so the interaction between the membrane and the membrane can occur through the gravitational field, thus affecting the distribution of matter. In 2003, shtanov et al. obtained a modified Friedmann equation with rebound solution through a 5-dimensional membrane world model ${ }^{[73]}$. In this model, the total action of gravity and matter can be written as:

$$
S=M^{3}\left[\int_{\text {bulk }}\left({ }^{5} R-2 \Lambda\right)+2 \int_{\text {brane }} K\right\rfloor+\int_{\text {brane }}\left(m^{2} R-2 \sigma\right)+\int_{\text {brane }} L\left(h_{a b}, \varphi\right)
$$

where ${ }^{5} R$ is the curvature scalar corresponding to the 5-dimensional background metric $g_{a b}, h_{a b}$ is the induced metric on the membrane, and $M$ and $m$ represent the 5-D and 4-D Planck masses, respectively. According to the least action principle, the constraint equation in bulk

$$
{ }^{5} G_{a b}+\Lambda g_{a b}=0
$$

and the dynamic equation on membrane

$$
m^{2} G_{a b}+\sigma h_{a b}+M^{3}\left(K_{a b}-K h_{a b}\right)=T_{a b}
$$

can be obtained respectively from the above formula. Since only the evolution on membrane is concerned, and the $h_{a b}$ in equation (14) is the RW metric of space homogeneity and isotropy and the case of $m=0$, it can be determined that the evolution of the universe satisfies the following equation:

$$
H^{2}+\frac{k}{a^{2}}=\frac{8 \pi G_{N} \rho}{3}-\frac{\rho^{2}}{M^{6}}+\frac{\Lambda_{e f f}}{3}
$$


here $\Lambda_{\text {eff }}=\frac{\Lambda}{2}-\frac{\rho^{2}}{3 M^{6}}, G_{N}=-\frac{3 \sigma}{4 \pi M^{6}}$. Therefore, in the simplest case without curvature term and cosmological constant term, we have

$$
H^{2}=\frac{8 \pi G_{N} \rho}{3}-\frac{\rho^{2}}{M^{6}}
$$

This result is similar to that in the loop quantum universe, both of which contain the correction of $\rho^{2}$. Therefore, when the density of matter on the brane is very high, it will produce a nonsingular rebound.

Both the semiclassical loop quantum universe model and the membrane world model only solve the singularity problem to a certain extent, which is a preparation for us to systematically introduce the repulsive force and high-dimensional superrepulsive force, and fundamentally solve the singularity problem of the universe.

By the analysis of inflation cosmology, when the inflation occurs, the energy scale of the universe is very high, which can be compared with the grand unification or even Planck energy scale. Therefore, when it comes to the calculation of the repulsion field of inflation, some appropriate corrections from quantum gravity are needed. The theory of nonperturbative superstring (or $\mathrm{m}$ ) points out that when the interaction distance of any physical process is very small, the following uncertainties should be met:

$$
\Delta t_{p} \Delta x_{p} \geq l_{s}^{2}
$$

This relationship is called the principle of spatiotemporal uncertainty (or noncommutative relationship of space-time, SSUR). Here $l_{s}$ represents the characteristic length of the string, while $\Delta t_{p} \Delta x_{p}$ describes the uncertainty of measurement for physical time and space coordinate. It is pointed out in references [47] and [48] that the non commutative relation of time and space is universally tenable. This conclusion is true not only for strings, but also for D-membrane.

In reference [74], the k-inflation model in noncommutative space-time is studied based on the uncertainty principle of space-time. The results show that in this model, all the disturbance modes are generated in the horizon, and the non commutative effect of time and space contributes to the power spectrum index of disturbance in the form of linear term. Compared with the experimental observation, it is found that the model can better meet the latest data. In our opinion, the pseudo vacuum energy considered by the inflationary universe model, the Higgs field energy considered by the standard physics model and the dark energy inferred by the observation cosmology are all related to the repulsive force that brings strong negative pressure, as shown in Figure 1 (Li Zongcheng, 2019).

Note that $M_{p l}^{-2}=8 \pi G=1$ is used for all units below this section.

Let $X=-\frac{1}{2} g^{\mu \nu} \nabla_{\mu} \varphi \nabla_{\nu} \varphi$ represent the kinetic energy term of a single scalar repulsion field, then the most general action used to describe the repulsion field can be expressed as:

$$
S=\int d^{4} X \sqrt{-g}\left[\frac{R}{2}+p(\varphi, X)\right],
$$

here $p(\varphi, X)$ is an arbitrary function of the repulsion field $\varphi$ itself and its kinetic energy as a scalar 
field, and the definition of $\mathrm{X}$ and the above actions ensure that the motion equation of the repulsion field is of second order. The energy momentum tensor of the repulsion field can be obtained by the variation of the action

$$
T_{\mu \nu} \equiv-\frac{2}{\sqrt{-g}} \frac{\delta S_{\varphi}}{\delta g^{\mu \nu}}=p_{X} \nabla_{\mu} \varphi \nabla_{\nu} \varphi+p g_{\mu \nu}, \quad p_{X} \equiv \frac{\partial p(\varphi, X)}{\partial X},
$$

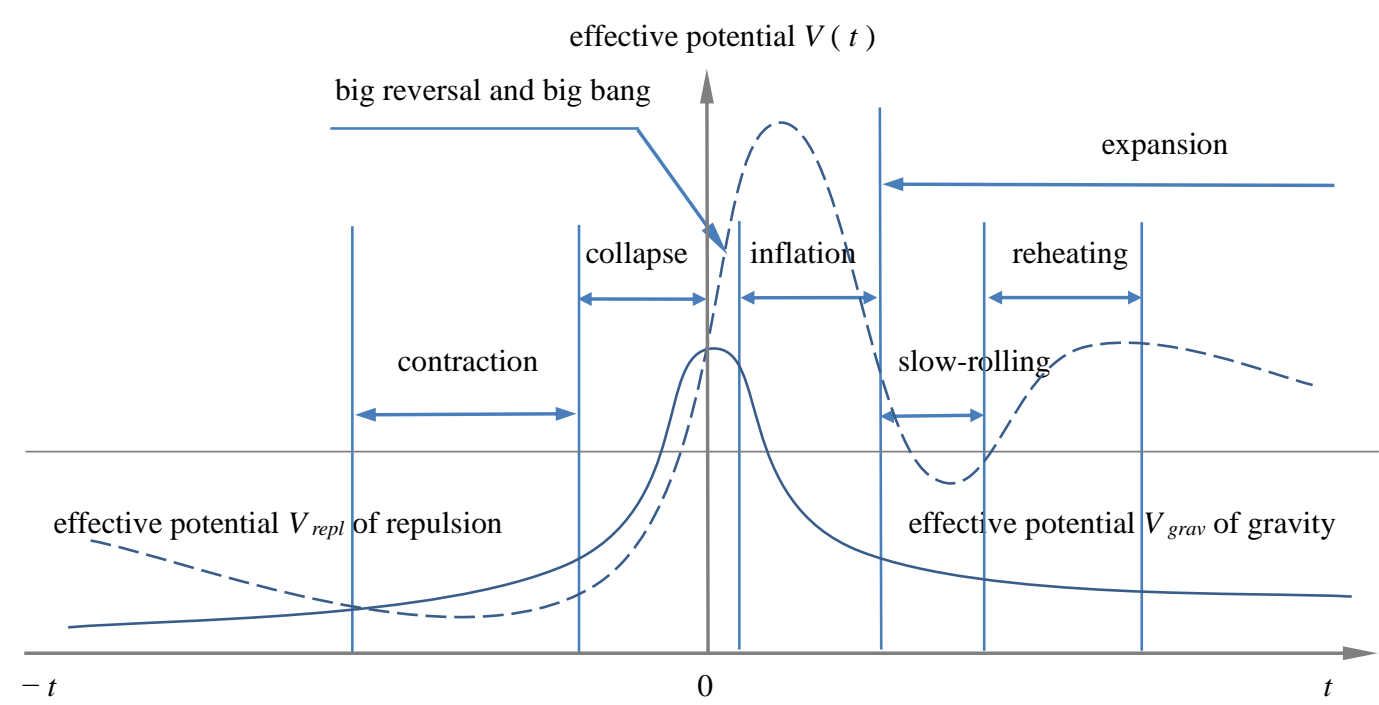

Figure 1 Gravity and Repulsion in the Universe

When the gravity is overwhelming for repulsion, the universe enters the stage of contraction and collapse.

When the repulsion is overwhelming for gravity, the universe enters the stage of inflation and expansion.

It is also equivalent to an ideal fluid, as long as its energy momentum tensor is expressed as $T_{\mu v}=(\rho+p) U_{\mu} U_{v}+p g_{\mu \nu}$. The $p$ appearing here is the pressure defined in the co-moving coordinate system, while the energy density defined in the same coordinate system is:

$$
\rho=2 X p_{X}-p
$$

In addition, the definition of four velocity $U_{\mu}$ is $U_{\mu}=-\nabla_{\mu} \varphi / \sqrt{2 X}$.

An uniform and homogeneous flat universe ( space curvature $K=0$ ) can be described by Friedmann-Robertson-Walker (FRW) metric. Its specific form is as follows:

$$
d s^{2}=-d t^{2}+a^{2}(t) d x^{2} .
$$

In this way, the kinetic energy of the repulsive field that determines the inflation of the universe is $X=\dot{\varphi}^{2} / 2$. The non commutative relation of time and space in formula ( 17 ) becomes:

$$
\Delta t \Delta x \geq l_{s}^{2} / a(t)
$$

The right of equation ( 22 ) is a function of time $t$, which is a variable in the period of $\Delta t$. Therefore, 
the value on the right side of the equation is uncertain. Thus, when $\Delta t$ is very large, the definition of formula ( 22 ) is invalid. If the conformal time $d \eta=d t / a$ is used, the same problem exists. In order to make equation ( 22 ) meaningful at any time, a new time coordinate $\tau: d \tau=a(t) d t$ must be introduced, and the scale changes accordingly:

$$
d s^{2}=-\frac{1}{a^{2}(\tau)} d \tau^{2}+a^{2}(\tau) d x^{2}
$$

Now, there is a good definition of non commutative relation between time and space: $\Delta \tau \Delta x \geq l_{s}^{2}$.

\section{Mechanism Framework for Offset of Cosmic Singularity}

It is necessary to point out clearly that it is an established fact that the universe avoids singularity before the big bang or in the big collapse, and it is not a mathematical and physical problem to be solved. The first thing we need to do now is to explore and reveal the internal mechanism of avoiding singularity in the collapse of the universe, and give a convincing natural and reasonable explanation.

To truly explain in a natural and reasonable way the offset of the cosmic singularity before the big bang, the accelerating expansion of the present universe, the irregularity of dark matter and dark energy, we consider noncommutative space-time, and will introduce the ultimate supersymmetry, the archeconjugation and the cluster resonance-at-cofrenquence. All kinds of strange phenomena converge into one possibility, that is, the closer they get to Planck scale, the stronger the conventional quantum effect, non commutative quantum effect, arche-conjugate effect and co-frequence cluster resonance effect are.

For the new physics set up in this series, the central concept and method are the arche-conjugation, while supersymmetry is the key concept and method formed around the arche-conjugation. Because, in our opinion, supersymmetry can't overcome the UV divergence in the quantization of supergravity, and the arche-conjugation introduced in this series of works can overcome the UV divergence of the quantization of supergravity.

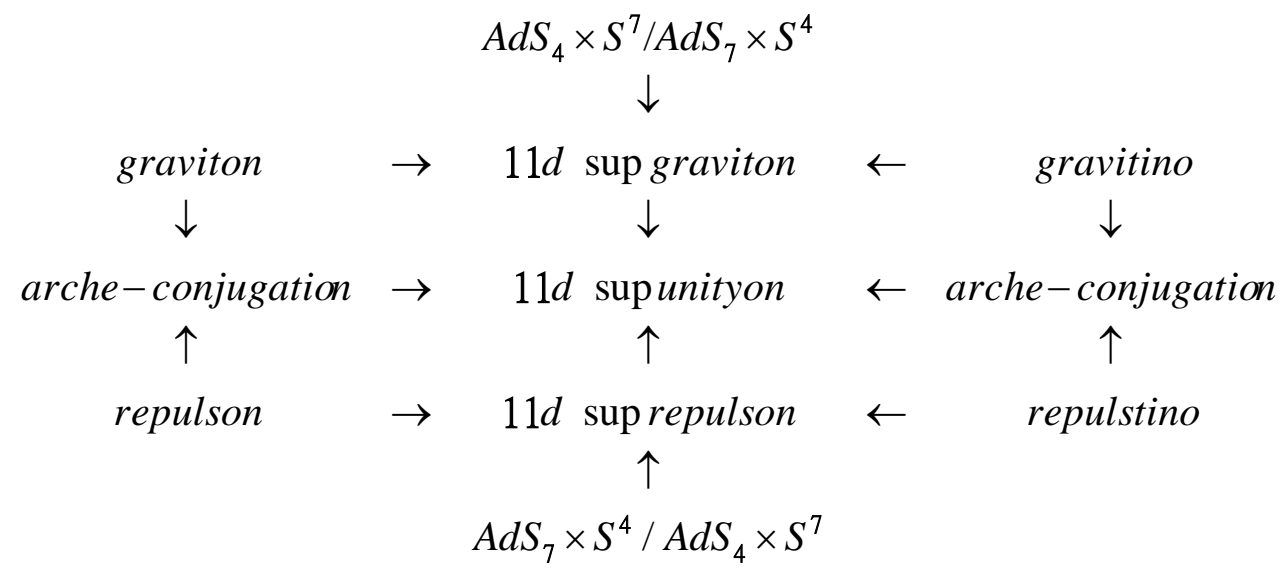

( 1 ) noncommutative time and space

For supergravity, dark energy and dark matter, we enter into supersymmetric space-time, quantum 
gravitational space-time (or Planck space-time), non-commutative quantum space-time, and will introduce the arche-pulsating space-time, which always exists and determines to affect the current and future world.

One of the characteristics of quantum mechanics is that in this theory coordinates and coordinates, momentum and momentum are commutative, while the relationship between coordinates and their conjugate momentum is non commutative. Some researches on string theory show that the coordinates are not commutative when they are close to Planck scale, and its relation satisfies

$$
\left[\tilde{x}^{\mu}, \tilde{x}^{v}\right]=i \alpha M^{\mu \nu}
$$

here $\alpha \in R, M^{\mu v}$ is an anti-symmetric matrix, $\tilde{x}$ is a noncommutative coordinate. In this way, common quantum mechanics becomes noncommutative quantum mechanics. In order to ensure that the system has rotational symmetry, it is necessary to have $\left[p^{\mu}, p^{\nu}\right]=0$, the noncommutative relationship between coordinates and momentum becomes

$$
[\tilde{x}, p]=i \sqrt{1-\alpha p^{2}}
$$

When $\alpha \rightarrow 0$, the theory goes back to quantum mechanics. In 2009, M. Battisti considered the non commutative geometry in Synder space ${ }^{[75]}$, and obtained the modified Friedmann equation through the quantum classical correspondence. The general process is relatively simple: under the classical limit, the commutation relation of operators should return to the form of Poisson bracket:

$$
-i[\tilde{q}, p] \rightarrow[q, p]=i \sqrt{1-\alpha p^{2}}
$$

Therefore, the relation a is satisfied for the commutative scale factor $a$ and its conjugate momentum $p_{a}$, where

$$
\begin{gathered}
\dot{a}=\frac{4 \pi G}{3} \frac{p_{a}}{a} \sqrt{1-\alpha p_{a}^{2}} \\
\dot{p}_{a}=\left(\frac{2 \pi G}{3} \frac{p_{a}^{2}}{a^{2}}+3 a^{2} \rho+a^{3} \frac{d \rho}{d a}\right) \sqrt{1-\alpha p_{a}^{2}}
\end{gathered}
$$

By the above two formulas and the Hamiltonian constraint $H_{N C} \approx 0$, we get the background evolution equation:

$$
H^{2}=\frac{8 \pi G}{3} \rho\left(1-\operatorname{sgn} a \frac{\rho}{\rho_{c}}\right)
$$

where the critical density is $\rho_{c}=\rho_{p}(2 \pi G / 3|\alpha|)$. When $\alpha \rightarrow 0, \rho_{c} \rightarrow \infty$ returns to the classical case. In the case of $\alpha>0$, Friedmann equation has a rebound solution.

\section{( 2 ) Ultimate Supersymmetry}

As a mathematical method, supersymmetry has very rich physical significance, which is related to superfield, hyperspace, super transformation and so on. In superfield, such as scalar superfield $\Phi(x, \theta)$, Bose field $\varphi$ (including all kinds of scalar field, vector field, tensor field, etc.) and Fermi field $\psi$ are included. There are all kinds of super gauge transformations between the two fields ${ }^{[76]-[78]}$. 
Now, in the super unifying force field $\Omega(X, \Theta)$ introduced here, we consider the gravitational field (especially the high-dimensional super gravitational field including Fermi field) $\Phi$ on the one hand, and the repulsive field (especially the high-dimensional super repulsive field including Bose field) $\Psi$ on the other hand. All kinds of supernormal transformations between gravitational field and repulsive field can be summarized as follows:

$$
\delta \Phi=A \vec{\varepsilon} \Psi, \quad \delta \Psi=-B \Phi \varepsilon,
$$

where, $\varepsilon$ is the rotation angle of the hyperparticle, as the major rotation of the anti commutation.

Suppose the multiplicity of sparticle is $\Omega=\left(\begin{array}{c}\Phi \\ i b \vec{\varepsilon} \Psi\end{array}\right)$, formula (29) becomes a

$$
\delta \Phi=-i A b^{-1}(i b \bar{\varepsilon} \Psi), \quad \delta(i b \bar{\varepsilon} \Psi)=-i b \bar{\varepsilon} B \Phi \varepsilon
$$

so

$$
\left(\begin{array}{c}
\Phi^{\prime} \\
i b \vec{\varepsilon} \Psi^{\prime}
\end{array}\right)=\left(\begin{array}{cc}
1 & -i A b^{-1} \\
-i b \dot{\varepsilon} B \varepsilon & 1
\end{array}\right)\left(\begin{array}{c}
\Phi \\
i b \vec{\varepsilon} \Psi
\end{array}\right)
$$

here $\varepsilon$ is commutative to the repulsive field $\Phi$ and anti-commutative to the gravitational field $\Psi$, then

$$
\alpha_{\mu v}=\left(\begin{array}{cc}
1 & -i A b^{-1} \\
-i b \bar{\varepsilon} B \varepsilon & 1
\end{array}\right)=\left(\begin{array}{ll}
1 & 0 \\
0 & 1
\end{array}\right)-i\left(\begin{array}{cc}
0 & A b^{-1} \\
b \bar{\varepsilon} B \varepsilon & 0
\end{array}\right) .
$$

The Lagrangian of super unified field is:

$$
L=\frac{1}{8}(\bar{D} D)^{2}\left(\Omega_{+} \Omega_{-}\right)-\frac{1}{2} \bar{D} D\left[V\left(\Omega_{+}\right)+V\left(\Omega_{-}\right)\right],
$$

where, $\Omega_{+}$and $\Omega_{-}, \bar{D}$ and $D$ are completely symmetrical.

For the quantum unified action from the interaction of quantum gravity and quantum repulsion, new variables are introduced: the square root $\sigma_{i}{ }^{a}$ of the three-dimensional metric and the potential $A_{a}{ }^{i}$ of the curvature self duality. The dynamic equation of the quantum unified action is as follows:

$$
\begin{gathered}
\dot{\tilde{\sigma}}^{a}=\sqrt{2}{ }^{ \pm} D_{b}\left(i \underline{T} \tilde{\sigma}_{\sigma}^{[b-a]}+T_{\sigma}^{[b-a]}\right), \\
{ }^{ \pm} A_{a}=\frac{1}{\sqrt{2}}\left(\left[i \underline{T} \tilde{\sigma}^{b},{ }^{ \pm} F_{a b}\right]-T^{b \pm} F_{a b}\right) .
\end{gathered}
$$

On the one hand, we consider the symmetry of supergravity ${ }^{[79]-[81]}$ : graviton vs gravitino; on the other hand, we introduce and consider the symmetry of superrepulsion: repulsion and repulstino.

Gravitons and gravitinos form supersymmetric relationships, which leads to the formation of high dimensional hypergravitons.

Repulsons and repulstinos form supersymmetric relationships, which leads to the formation of high dimensional hyperrepulsons. 


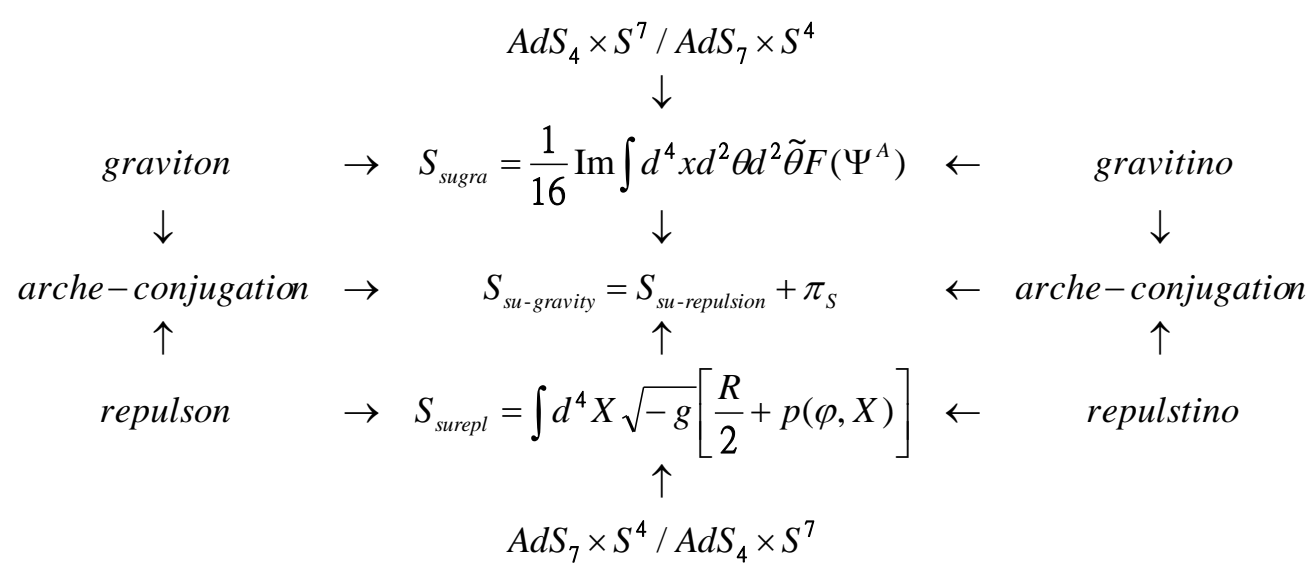

Furthermore, between the high-dimensional supergraviton and the high-dimensional superrepulson, we introduce the high-dimensional supersymmetric arche-conjugate pulsaton (super coupling).

On the one hand, the quantum aspect of dark matter is attributed to quantum gravitons, on the other hand, the quantum aspect of dark energy is attributed to quantum repulsons.

Furthermore, we introduce and analyze the quantum conjugate (super coupling) pulsaton between the quantum aspect of dark matter as a quantum graviton and the quantum aspect of dark energy as a quantum repulson.

The application of ultimate supersymmetry to unified action theory leads to the introducing of ultimate unified action. The ultimate supersymmetry includes gravitational supersymmetry and repulsive supersymmetry, in which the gravitational supersymmetry is a theory constructed by adding a graviton ( whose $\operatorname{spin} s=2$ ) as a boson to a gravitino ( whose $\operatorname{spin} s=3 / 2$ ) as a Fermi partner ${ }^{[81]-[83]}$, while the repulsive supersymmetry is a theory constructed by adding a repulson to a repulstino.

( 3 ) Arche-Conjugation

We will not only consider the dynamic dark energy and the interacting dark energy, but also introduce and consider the arche-conjugate dark energy.

We will not nonly consider the dynamic dark matter and the interacting dark matter, but also introduce and consider the arche-conjugate dark matter.

Let $V$ be the volume of space, and the action of scalar disturbance can be given by the formula:

$$
S=\frac{V}{2} \int_{k<k_{0}} d \eta d^{3} k \approx z^{2}(\eta)\left(\zeta_{-k}^{\prime} \zeta_{k}^{\prime}-c_{s}^{2} k^{2} \zeta_{-k} \zeta_{k}\right),
$$

where the apostrophe represents the differential of the new time coordinate $\eta$, which is defined as:

$$
\frac{d \eta}{d \tau} \equiv a_{e f f}^{-2}=\left(\frac{\beta_{k}^{-}}{k_{+}^{\beta}}\right)^{1 / 2}=\frac{1}{a^{2}}(\tau-\Delta \tau),
$$

$\beta_{k}^{ \pm}(\tau)$ functions are defined here:

$$
\beta_{k}^{ \pm}(\tau)=a^{ \pm 2}(\tau-\Delta \tau), \text { and } \Delta \tau=l_{s}^{2} k
$$

where $\zeta_{k}$ is the curvature disturbance and $z \equiv a \sqrt{2 \varepsilon_{1}} / c_{s}$ is Mukhanov variable. The $\beta_{k}^{ \pm}(\tau)$ function used here is equivalent to the formula used in other literature in the sense of integral, and once the exact 
solution of the perturbed equation is obtained, the form of the $\beta_{k}^{ \pm}(\tau)$ function will not affect the final physical results. However, if the present form is chosen, the effect of non commutation of space-time can be highlighted to the greatest extent under the approximate conditions, and the perturbed equation will become easier to deal with. The equation of motion satisfied by the variable $\zeta_{k}$ is:

$$
u_{k}^{\prime \prime}+\left(c_{s}^{2} k^{2}-\frac{z^{\prime \prime}}{z}\right) u_{k}=0
$$

This equation can be obtained from the variation of action (35), where the modulus function is defined as $u_{k}=z \zeta_{k}$. In the case of slow-rolling approximation, equation (38) is simplified to :

$$
u_{k}^{\prime \prime}+\left(c_{s}^{2} k^{2}-\frac{v^{2}-1 / 4}{\eta^{2}}\right) u_{k}=0
$$

After the first order terms of slow roll parameter and $\lambda$ are retained, the following results are obtained:

$$
v \approx \frac{3}{2}+\varepsilon_{1}+\frac{\varepsilon_{2}}{2}-s-\frac{2}{3} \lambda .
$$

The motion equation of tensor perturbation is almost the same as that of scalar perturbation, but the definition of Mukhanov variable becomes $z=a$. The specific disturbance equation is as follows:

$$
v_{k}^{\prime \prime}+\left(k^{2}-\frac{a^{\prime \prime}}{a}\right) v_{k}=0,
$$

where the modular function is defined as $v_{k} \equiv a h k / 2$. It should be noted that $h_{k}$ here represents two physical freedom degrees of tensor perturbation, namely $h_{+}$and $h_{x}$. Using the same approximation as scalar perturbation, we get:

$$
\frac{a^{\prime \prime}}{a} \approx \frac{1}{\eta^{2}}\left(1-\varepsilon_{1}\right)^{-2} \Sigma^{-2}(\tau, \Delta \tau)\left[2 \Sigma(\tau, \Delta \tau)-\varepsilon_{1}\right] \approx \frac{v^{2}-1 / 4}{\eta^{2}},
$$

leave the value of $v$ to the linear term:

$$
v \approx \frac{3}{2}+\varepsilon_{1}-\frac{2}{3} \lambda
$$

To define conjugate momentum and its quantization:

$$
\begin{gathered}
\pi_{k}=\mu_{k}^{\prime}-\frac{a^{\prime}}{a} \mu_{k}, \\
\mu_{k}(\eta)=\frac{1}{\sqrt{2 k}}\left(a_{k}(\eta)+a_{-k}^{\dagger}(\eta)\right), \\
\pi_{k}(\eta)=-i \sqrt{\frac{k}{2}}\left(a_{k}(\eta)-a_{k}^{\dagger}(\eta)\right) .
\end{gathered}
$$

Under the special conformal time $\eta_{0}$, by the Bogoliubov transformation, we have:

$$
a_{k}(\eta)=u_{k}(\eta) a_{k}\left(\eta_{0}\right)+v_{k}(\eta) a_{-k}^{\dagger}\left(\eta_{0}\right),
$$




$$
a_{k}(\eta)=u_{k}(\eta) a_{-k}^{\dagger}\left(\eta_{0}\right)+v_{k}(\eta) a_{k}\left(\eta_{0}\right)
$$

So in $\mu_{k}, \pi_{k}$, there should be

$$
\begin{gathered}
\mu_{k}(\eta)=f_{k}(\eta) a_{k}\left(\eta_{\mathbf{0}}\right)+f_{k}^{*}(\eta) a_{-k}^{\dagger}\left(\eta_{\mathbf{0}}\right), \\
\pi_{k}(\eta)=-i\left(g_{k}(\eta) a_{k}\left(\eta_{\mathbf{0}}\right)\right)+g_{k}^{*}(\eta) a_{-k}^{\dagger}\left(\eta_{0}\right) .
\end{gathered}
$$

In fact, the arche-conjugation introduced in this series of work is the requirement of super dynamic equilibrium, that is to say, the arche-conjugation is the fundamental requirement for the natural formation of the universe between high-dimensional supergravity and high-dimensional superrepulsion, between quantum gravity and quantum repulsion, as well as between classical gravity and classical repulsion.

For the high-dimensional super unified action between the high-dimensional supergravity and the high-dimensional superrepulsion, the quantum unified action between the quantum gravity and the quantum repulsion, and the classical unified action between the classical gravity and the classical repulsion, the disequilibrium is the actual form (normal state), the equilibrium is the ideal form (abnormal state), therefore, in the normal state, the degree of deviation of the equilibrium is non-zero; in the abnormal state, the degree of deviation of the equilibrium is zero. Imbalance always drives the super forces to form deviation, while equilibrium always requires the super forces to shorten the deviation. Here are three situations:

( a ) $V_{\text {sugra }}(\Phi)-V_{\text {surepl }}(\Phi)=0$,

the effective potential of supergravity and that of superrepulsion reach equilibrium;

( b ) $V_{\text {sugra }}(\Phi)-V_{\text {surepl }}(\Phi) \leq 0$,

the effective potential of supergravity is less than or equal to that of superrepulsion;

(c) $V_{\text {sugra }}(\Phi)-V_{\text {surepl }}(\Phi)>0$,

the effective potential of super gravity is larger than that of super repulsion.

In the super-inflationary universe model of ultra-synergy physics established later in this series, a mechanism (the quantum statistical physics process of nonlinear non-equilibrium) that combines the generation of baryon asymmetry with the super-inflation and reheating process of the universe will be studied.

( 4 ) Cluster Resonance-at-Cofrenquence

Now, a basic inference is given: at the level close to quantum gravity and quantum repulsion, all the gravitons and gravitinos, as well as all the repulsons and repulstinos, are in the cluster resonance -at-cofrenquence state.

In our opinion, a series of major knotty problems, such as the singularity, inflation and accelerated expansion of the universe, involve super gravity, dark energy and dark matter. Super gravity, dark energy and dark matter are three closely related problems. In essence, dark energy and dark matter are problems of quantum gravity and quantum repulsion.

The coupling of dark matter and dark energy is attributed to the interaction of quantum gravity and 
quantum repulsion.

The decoupling of dark matter and dark energy is attributed to the separation of quantum gravity and quantum repulsion.

\section{High-dim Supergravity and High-dim Superrepulsion}

Based on the previous research in this series, It can be seen that gravitation, strong action and weak action are all quantum gravitation in essence. The unity of four basic physical interactions is not the ultimate unity. For super gravity, dark energy and dark matter, it is not enough to have only four basic physical interactions.

In addition to the quantum gravitation, there is a need for further research, especially in the quantum repulsion. We should not only deeply and systematically study the unity between classical gravity and classical repulsion, but also the unity between quantum gravity and quantum repulsion, especially the unity between high-dimensional supergravity and high-dimensional superrepulsion.

According to the viewpoint of arche-unity physics established in this series of works, only the unity between high-dimensional supergravity and high-dimensional superrepulsion is the ultimate unifying force (UUF) of the universe.

There are still many problems in the research of supergravity ${ }^{[83]-[87]}$. The main topics include the supergravity of shell, off-shell and hyperspace, the coupling with matter, the extended supersymmetry, Kaluza Klein (KK) reduction (the only complete non-linear compact in the gravitational space $A d S_{4} \times$ $S^{7}$ ), and the application of phenomenology, such as the theory of embedded string, etc. Previous studies have shown that the 11-d super gravity has $N \sim 8$ (maximum) supersymmetry. From the residual minimal supersymmetry in the englert solution of the 11-dimensional supergravity, we can find a mechanism of the so-called "spontaneous compactness" of $d=11$ supergravity ${ }^{[77]-[79]}$. In this framework, 11-dimensional hyperspace is compactly transformed into $M_{4} \times N_{r}$, where $M_{4}$ is 4-dimensional $A d S$ (anti de Sitter) space-time and $N_{r}$ is a compact (Lie group) manifold. The form of residual supersymmetry and internal canonical symmetry after spontaneous compact is closely related to the topological properties of compact 7-manifold $N_{r}$. The fundamental fermions and gauge fields with mass obtained by Higgs mechanism actually constitute the harmonic expansion in Kaluza Klein reduction. It is noted that there are two breaking scales of the same or the same order of magnitude: complete SUSY breaking scale $M_{S U S Y}$ and internal symmetry breaking scale $M_{\text {gauge }}$. The former is generally believed to be the minimum scale (generally very high) that makes the nature not show the symmetry of Bose and Fermi, while the latter is a direct low-energy spectrum. It is difficult to imagine that they have the same order of magnitude! A clever way to solve this problem is to make the high-order terms (spin 3/ 2, 2 and other Hypergravitation propagators) "decouple" in K-K harmonics, but only to make the fermions and gauge fields in the usual SU (3) $\times \mathrm{S} \mathrm{U}(2) \times \mathrm{U}$ (1) model coupled with Higgs particles, so that mgauge can be indirectly depressed. This means that the supergravity is at least partially preserved, not completely broken.

In the existing studies ${ }^{[82]-[85]}$, it can be seen that $N=1$ supergravity in $11 \mathrm{~d}$ is the only one, because it is the largest dimension that we can enhance $N=8$ supergravity in $4 \mathrm{~d}$, which is the maximum super gravity with spin $\leq 2$ (for higher spin, there is no known way to interact with a limited number of fields). In a higher dimension, the eight gravitini of $4 \mathrm{~d}$ will only form a part of a graviton. In 10 
dimensions, there are two possible maximum gravitation, that is, when $N=2$, IIA has two different kinds of gravitini and IIB has two kinds of gravitini with the same chirality. They correspond to the low energy limits of the IIA and IIB string theory. IIA supergravity is obtained by the periodic reduction of $11 \mathrm{~d}$ supergravity with $N=1$ (11d super gravitinos split into two different chiral $10 \mathrm{~d}$ gravitini), but IIB is obtained without reduction of any dimension (although in the full string theory, it is related to $11 \mathrm{~d}$ supergravity without disturbance). Therefore, $11 \mathrm{~d}$ and IIB $10 \mathrm{~d}$ supergravity are important examples of supergravity, from which we can get the rest. The biggest supersymmetric background of these two theories is: for 11d super gravity, Minkowski, $A d S_{4} \times S^{7}, A d S_{7} \times S^{4}$ and PP (parallel plane) are obtained as Penrose limit of $A d S_{4} \times S^{7}$ and $A d S_{7} \times S^{4}$. For II B super gravity, there are Minkowski, $A d S_{5} \times S^{5}$ and PP waves as Penrose limit of $A d S_{5} \times S^{5}$. Therefore, the nontrivial cases related to compactness (except that the PP wave is only limited) are $A d S_{4} \times S^{7}, A d S_{7} \times S^{4}$ and $A d S_{5} \times S^{5}$.

Now, we introduce the hyperrepulsion which forms the arche-conjugate relation with hypergravity. As a correspondence of arche, 11 dimen superrepulsion also has $M \sim 8$ (maximum) supersymmetry. From the residual minimal supersymmetry in the englert solution of the 11 dimensional superrepulsion, we can also find a mechanism of the so-called "spontaneous extension" $d=11$ superrepulsion. The form of residual supersymmetry and internal gauge symmetry after spontaneous extension is closely related to the topological properties of the extended 4-manifold $M_{4}$. The fundamental fermions and gauge fields with mass obtained by Higgs mechanism actually constitute the harmonic expansion in Kaluza Klein reduction. To reduce the huge difference in the scale of decimal, the "decoupling" formed by the high-order terms (the propagator of supergravity with spin 3 / 2, 2 and other ) in K-K harmonics makes the fermions and gauge fields in the $\mathrm{SU}(3) \times \mathrm{S} \mathrm{U}(2) \times \mathrm{U}(1)$ model coupled with Higgs particles, so that mgauge can be indirectly depressed. This means that the effectiveness of super repulsion is limited in a limited dimension.

In a higher dimension, corresponding to 8 gravitini of $4 \mathrm{D}$, we can introduce 8 repulstini, which only form a part of a repulstino. In 10 dimensions, corresponding to the existence of two possible maximum supergravity, there should be two possible maximum superrepulsion, that is, when $N=2$, IIA has two different chiral repulstini, and IIB has two identical chiral repulstini. They correspond to the low energy limits of the IIA and IIB string theory. IIA supergravity and IIA superrepulsion form the arche-conjugate correlation, thus forming IIA super unified action. 11d superunifying force and IIB 10d superunifying force are important examples of super unifying force, from which we can get other super unifying forces.

As a correspondence of the arche, the self propagating $d=11$ superrepulsion can be reduced to a scalar. In the ultimate unification of cosmic superactions, the largest supersymmetric background of the theory is:

For 11d supergravity, 11d superrepulsion and 11d superunifying force, Minkowski, $A d S_{4} \times S^{7}$, $A d S_{7} \times S^{4}$ and PP (parallel plane) are obtained as Penrose limit of $A d S_{4} \times S^{7}$ and $A d S_{7} \times S^{4}$.

For II B supergravity, II B superrepulsion and II B superunifying force, we have Minkowski, $A d S_{5}$ $\times S^{5}$ and PP waves as Penrose limits of $A d S_{5} \times S^{5}$. Therefore, the nontrivial case related to compactness (except that the PP wave is only limited) is $A d S_{4} \times S^{7}, A d S_{7} \times S^{4}$ and $A d S_{5} \times S^{5}$.

In $4 \mathrm{D}$, for $N=2$ supersymmetry, we have $N=2$ vector multiplet, which is composed of $N=1$ vector $W_{\alpha},(1,1 / 2)$ (vector plus spinor), plus $N=1$ chiral multiplet, and ( $\left.1 / 2,0\right)$ (spinor plus scalar). We can also get the hypermultiplet of $N=2$, which is composed of two chiral multiples $Q$ and 
$\tilde{Q}$. For $n N=2$ vector multiple states $\Psi^{A}$, when $A=1, \cdots, n$, we can associate the action of $N=2$ hyperspace gravitational potential $F\left(\Psi^{A}\right)$

$$
S=\frac{1}{16 \pi} \operatorname{Im} \int d^{4} x d^{2} \theta d^{2} \tilde{\theta} F\left(\Psi^{A}\right)
$$

with the action of repulsion in the following formula:

$$
\frac{1}{16 \pi} \operatorname{Im} \int d^{4} x d^{2} \theta d^{2} \tilde{\theta} F\left(\Psi^{A}\right)=\int d^{4} X \sqrt{-g}\left[\frac{R}{2}+p(\varphi, X)\right]+\pi_{S}
$$

We can write the repulsive action in $N=1$ :

$$
\operatorname{Im} \int d^{4} x\left[\int d^{2} \theta F_{A B}(\Phi) W^{A \alpha} W_{\alpha}^{B}+\int d^{2} \theta d^{2} \tilde{\theta}\left(\Phi^{\dagger} e^{-2 g V}\right)^{A} F_{A}(\Phi)\right]
$$

where

$$
F_{A}(\Phi)=\frac{\partial F}{\partial \Phi^{A}}, \quad F_{A B}=\frac{\partial^{2} F}{\partial \Phi^{A} \partial \Phi^{B}}
$$

To do this, use the $N=1$ hyperspace language:

$$
\int d^{2} \theta d^{2} \tilde{\theta}\left(\left(Q^{\dagger} e^{-2 g V}\right)_{i} Q_{i}+\left(\tilde{Q} e^{2 g V}\right)_{i} \tilde{Q}_{i}\right)+\int d^{2 \theta}\left(\sqrt{2}(\tilde{Q} \Phi)_{i} Q_{i}+m_{i} \tilde{Q}_{i} Q_{i}\right)+\text { h.c. },
$$

We give a coupling for $m$ hypermultiplets $i=1, \cdots, m$ with standard dynamical terms, where the interaction term between hyperspace and vector must also comply with global invariance, but we have not clearly explained how to achieve this. In general, $Q$ and $\tilde{Q}$ can also have a general dynamics term from their own Kahler potential.

The Lagrangian dynamics term of vector multiplets is

$$
L=g_{A \bar{B}} \partial_{\mu} X^{A} \partial^{\mu} \bar{X}^{\bar{B}}+g_{A \bar{B}} \bar{\lambda}^{i A} \partial \lambda_{i}^{\bar{B}}+\operatorname{Im}\left(F_{A B} \Xi_{\mu \nu}^{-A} \Xi_{\mu \nu}^{-B}\right),
$$

where

$$
\begin{aligned}
& g_{A \bar{B}}=\partial_{A} \partial_{\bar{B}} K, \\
& K(X, \bar{X})=i\left(\bar{F}_{A}(\bar{X}) X^{A}-F_{A}(X) \bar{X}^{A}\right), \\
& F_{A}(X)=\partial_{A} F(X), \quad F_{A B}=\partial_{A} \partial_{B} F(X),
\end{aligned}
$$

and $A, B=1, \cdots, n$.

The properties of the particle field can be expressed by Bose coordinates $x_{u}$ and Fermi coordinates $\Psi^{i \alpha}$. They are Mozorana spinors satisfying the anti-commutation relation. Their supersymmetry and internal symmetry are transformed into

$$
\delta \Psi^{i \alpha}=R_{\mu \nu} \pi^{i \alpha}, \quad \delta_{I} \Psi^{i \alpha}=-i I^{i} E_{j} \Psi^{j \alpha} .
$$

By the quantum field theory, the Lagrangian of gauge field and matter field can be taken as 


$$
L_{0}=-\frac{1}{4} R_{\mu \nu}^{A B} R_{A B}^{\mu \nu}+\frac{1}{2} \Psi_{i \alpha x} \Psi^{i \alpha}
$$

where

$$
R_{\mu \nu}^{A B} R_{A B}^{\mu v}=R_{\mu \nu}^{a b}(M) R_{a b}^{\mu \nu}(M)+R_{\mu \nu}^{i}(E)+R_{\mu \nu}^{i \alpha}(H) R_{i \alpha}^{\mu \nu}(H),
$$

The torsion free condition $R_{\mu \nu}^{a}(\rho)=0$ is used here.

Taking the Lagrangian of supergravity in the form of ERS

$$
L_{s g}=-\frac{1}{4 k^{2}} e k-\frac{1}{2} \varepsilon^{\mu \nu \lambda \rho} \Lambda_{\mu}^{i \alpha} \gamma_{5} \gamma_{v} D_{\lambda}^{\prime} \Lambda_{\rho i \alpha},
$$

where the first term is Einstein gravity term, the second term is Rarita Schwinge term, and

$$
D_{\lambda}^{\prime}=\partial_{\lambda}-B_{\lambda}^{a b}\left(\sigma_{a b}\right)^{T}
$$

Supersymmetry: the first and second terms of formula (57)

$$
\begin{aligned}
\delta_{S} L_{(1)} & =\delta_{S}\left(\frac{1}{16 K} \varepsilon^{\mu \nu \lambda \rho} V_{\mu}^{\mathrm{a} a} V_{v}^{b} R_{\lambda \rho}^{c d}(M)\right)=\frac{i}{8 K} \varepsilon^{\mu \nu \lambda \rho} \varepsilon_{a b c d} \pi^{i \alpha} \Lambda_{\mu}^{i \beta}\left(\gamma_{c}^{a}\right)_{\alpha \beta} V_{v}^{b} R_{\lambda \rho}^{c d}(M), \\
\delta_{S} L_{(2)} & =\delta_{S}\left(-\frac{1}{4} \varepsilon^{\mu \nu \lambda \rho} \Lambda_{\mu}^{i \alpha} \gamma_{5} \gamma_{v} R_{\lambda \rho}^{i \alpha}(H)\right) \\
& =-\frac{1}{4} \varepsilon^{\mu \nu \lambda \rho}\left[\delta_{S} \Lambda_{\mu}^{i \alpha} \gamma_{5} \gamma_{v} R_{\lambda \rho}^{i \alpha}(H)+\Lambda_{\mu}^{i \alpha} \gamma_{5} \gamma_{b} R_{\lambda \rho}^{i \alpha}(H) \delta_{S} V_{v}^{b}+\Lambda_{\mu}^{i \alpha} \gamma_{5} \gamma_{v} \delta_{S} R_{\lambda \rho}^{i \alpha}(H)\right] .
\end{aligned}
$$

Previous studies have shown that spontaneous compactness of $d=11$ supergravity results in the following Einstein equation solutions (in the form of Castellani and Warner ${ }^{[88]-[90]}$ ):

$$
F_{\alpha_{1}-\alpha_{4}}= \pm \sqrt{3} e Y_{\alpha_{1}}^{\pi_{1}}(z) Y_{\alpha_{2}}^{\pi_{2}}(z) Y_{\alpha_{3}}^{\pi_{3}}(z) Y_{\alpha_{4}}^{\pi_{4}}(z) T_{\pi_{1}-\pi_{4}},
$$

Here, the 4-spacetime component and the mediocre (due to the existence of parallelism) Ricci curvature are not written. In the formula (60), index $\pi$ is taken as $1, \cdots, 8, T_{\pi 1-\pi 4}$ is the constant value anti symmetric $S O(8)$ tensor, $Y(z)$ as the $S O(8)$ positive definite matrix, which is parameterized by the 7-spherical coordinate $z$. $T$ characterizes the (inverse) self-dual property of solution $F$, and by the field equation, it should satisfy the following relation with tensor $\Gamma$ of $S O$ (7):

$$
T_{\pi_{1}-\pi_{4}}=\xi \Gamma_{\pi_{1}-\pi_{4}} \xi
$$

Here $\xi$ is the constant rotation of $S O$ (7).

It is suggested in reference [88] that $T_{\pi 1-\pi 4}$ is not linearly independent as a $S O$ (8) tensor. Therefore, a set of $S O(8)$ rotation invariant bases can be selected to construct all 8-parameter clusters. This enables us to use the solution manifold (60) instead of $R^{8}$, and use a quotient space (manifold) of the automorphism group $G_{2}$ of (60) to approach $S^{7}$, so that the discussion of the supersymmetry property of the solution can be transformed into the study of the (supersymmetric) group transformation property of $G_{2} / K$ under the ${ }^{-} \operatorname{Spin}(7)$ transformation (parallelism). On $S^{7}$, it is generally believed that all supersymmetries are broken by ${ }^{-}$Spin ( 7 ).

We note that in the Kaluza Klein theory of supersymmetry, it has been discussed by Kerner ${ }^{[88]-[90]}$ 
to directly construct 4-dimensional "super" manifolds by means of order Lie groups instead of the Majorana spinor coordinates of usual super space. Kerner's idea is to equate the $G$-adjoint representation of non Abelian K-K bundle $P\left(M_{4} \times \Lambda\{\theta\}, G\right)$ with $Z_{2}$-principle bundle on $M_{D}$. By the realization of embedding: $\mathscr{C}_{G} \rightarrow a d\left(. /{ }_{G}\right) \rightarrow . / S O(\mathrm{~N})$, all Dirac spinors can be decomposed into direct sums of irreducible multiplets ${ }^{[00]-[92]}$ on a certain submanifold. Therefore, for a certain subgroup at least, there is no basic (irreducible) spinometer showing $\psi^{A}$.

The group transformation properties of $G_{r} / K$ under $8 \rightarrow 7 \oplus 1^{-}$Spin ( 7 ) decomposition induced by parallelism are investigated. Obviously, we should take $S O$ (4) in particular, that is, $\operatorname{dim} \operatorname{dim} G_{2} / S O$ ( 4 ) 8, the basic representation dimension of $N=8$ internal symmetric group. Consider (transform) group space $X_{p}$, whose generalized coordinate is $\eta^{\alpha}$. The so-called ( \pm ) parallelism refers to such a set of "parallel vector displacements".

$$
\text { (+) }: \mathrm{TUSU}^{-1}=T_{1} U S_{1} U^{-1} ;(-): U T U^{-1} S=U T_{1} U^{-1} S_{1},
$$

Here, point pair T, S, $T_{1}, S_{1}$ are the element of group, and as the point in $X_{p}, T, S, T_{1}, S_{1}$ are respectively connected by their own geodesic lines, and the linear connections defined thereby are $\operatorname{recorded}$ as $\stackrel{+}{\Gamma_{\gamma \beta}^{\alpha}}$ and $\stackrel{-}{\Gamma_{\gamma \beta}^{\alpha}}$.

\section{Quantum Hedge-Expansive Universe Equation}

Under the big unified framework of quantum gravitational field and quantum repulsive field, this series of work comprehensively expands the general relativity and loop quantum ring gravitation theory, establishes the quantum repulsive field equation against the quantum gravitational field equation, and then establishes the quantum hedge-unified field equation describing the interaction of quantum gravitational field and quantum repulsive field; On this basis, the quantum repulsive universe equation against the quantum gravitational universe equation is established, thus a set of new equations describing the expansive universe model under the condition of fully considering the hedge effect of quantum gravitational field and quantum repulsion field, which can be called the expansive universe equation of quantum hedge-unified field theory, is established.

( 5.1 ) Friedmann equation related to loop quantum gravity

For the classical flat FRW universe (Friedmann-Robertson-Walker), the Hamiltonian constraint of its gravitational action can be simply expressed as

$$
H_{c l, g r a v}=-\frac{3}{8 \pi G \gamma^{2}} \sqrt{p_{\text {grav }}} c^{2}+H_{M}
$$

where the momentum of gravity $p_{\text {grav }}=1 / a^{2}, c=\gamma \dot{a}, \gamma \approx 0.2375, a$ is the scale factor, and $H_{M}$ is the Hamiltonian of the gravitational mass. Here, define the energy density: $\pi \equiv \frac{E}{V}=\frac{H_{M}}{a^{3}}$.

If $H_{M} \approx 0$, the Friedmann equation related to gravity can be obtained in the case of $k=0$ (in the FRW scale, $k=0$ corresponds to a flat universe): 


$$
\dot{H}_{\text {grav }}=-4 \pi G\left(p_{\text {grav }}+\rho\right)+\frac{k}{a^{2}}
$$

here, when $a \rightarrow 0$, the energy density is divergent, which is the singularity moment of the big bang. When the loop quantum gravity effect cannot be ignored, the Hamiltonian constraint of the original gravity is modified as follows:

$$
H_{Q, \text { grav }}=-\frac{3}{8 \pi G \gamma^{2} \bar{\mu}^{2}} \sqrt{p} \sin ^{2}(\bar{\mu} c)+H_{M}
$$

where $\bar{\mu}$ is the length of the loop that makes up the smallest facet. When $\bar{\mu} \rightarrow 0$, there is $\sin ^{2}(\bar{\mu} c) / \bar{\mu}^{2} \rightarrow 1$, then $H_{Q, \text { grav }} \rightarrow H_{c l, \text { grav }}$ will return to the classical limit. Through the Hamiltonian equation related to quantum gravity:

$$
\dot{p}_{\text {grav }}=\left\{p_{\text {grav }}, H_{Q, \text { grav }}\right\}=-\frac{8 \pi G \gamma}{3} \frac{\partial H_{Q, \text { grav }}}{\partial c}=\frac{2 a}{\gamma \bar{\mu}} \sin (\bar{\mu} c) \cos (\bar{\mu} c)
$$

By using $p_{\text {grav }}=1 / a^{2}$ as a substitution, the evolution of scale factor with time can be obtained:

$$
\dot{a}=\frac{1}{\gamma \bar{\mu}} \sin (\bar{\mu} c) \cos (\bar{\mu} c)
$$

On the other hand, by the Hamiltonian constraint $H_{Q, \text { grav }} \approx 0$, we can get:

$$
\sin ^{2}(\bar{\mu} c)=\frac{8 \pi G \gamma^{2} \bar{\mu}^{2}}{3 a} H_{M}
$$

By combining the above two formulas, the modified Friedmann equation related to gravity is obtained:

$$
H_{\text {grav }}^{2}=\frac{8 \pi G}{3} \rho\left(1-\frac{\rho}{\rho_{c}}\right)
$$

where the critical density is

$$
\rho_{c}=\frac{\sqrt{3}}{16 \pi^{2} \gamma^{3}} \rho_{p}, \quad \rho_{p}=G^{-2} \text { is the first density of Planck. }
$$

The appearance of the critical density $\rho_{c}$ is completely a quantum gravitational effect.

( 5.2 ) the non fiedmann equation related to the quantum repulsion of a loop

Corresponding to the regular form of the loop quantum gravitational universe, we also use Hamiltonian language to discuss the loop quantum repulsive universe. For the classical flat FRW universe (Friedmann-Robertson-Walker), the Hamiltonian constraint of repulsion can be simply expressed as

$$
H_{c l, r e p l}=-\frac{3 G}{8 \pi} \gamma^{2} \sqrt{p_{r e p l}} c^{2}+H_{E}
$$


where, the momentum of repulsion $p_{\text {repl }}=a^{2}, c=\gamma \dot{a}, \gamma \approx 0.2375, a$ is the scale factor, and $H_{E}$ is the Hamiltonian of the repulsive energy. Here, define the energy density: $\pi \equiv \frac{E}{V}=\frac{H_{E}}{a^{3}}$.

If $H_{E} \approx 0$, the non Friedmann equation related to repulsion can be obtained in the case of $k=0$ (in the FRW scale, $k=0$ corresponds to a flat universe):

$$
\dot{H}_{\text {repl }}=-\frac{4 \pi}{G}\left(p_{\text {repl }}+\sigma\right)+k a^{2}
$$

here, when $a \rightarrow 0$, the energy density is divergent, which is the singularity moment of the big bang. When the loop quantum repulsion effect cannot be ignored, the Hamiltonian constraint of the original repulsion is modified as follows:

$$
H_{Q, \text { repl }}=-\frac{3 G \bar{\mu}^{2}}{8 \pi} \gamma^{2} \sqrt{p_{\text {repl }}} \sin ^{2}\left(\frac{1}{\bar{\mu}} c\right)+H_{E}
$$

where $\bar{\mu}$ is the length of the loop that makes up the smallest facet. When $\bar{\mu} \rightarrow 0$, there is $\sin ^{2}\left(\frac{1}{\bar{\mu}} c\right) \bar{\mu}^{2} \rightarrow 1$, then $H_{Q, r e p l} \rightarrow H_{c l, r e p l}$ will return to the classical limit. Through the Hamiltonian equation related to quantum repulsion:

$$
\dot{p}_{\text {repl }}=\left\{p_{\text {repl }}, H_{Q, \text { repl }}\right\}=-\frac{8 \pi}{3 G \gamma} \frac{\partial H_{Q, \text { repl }}}{\partial c}=2 \operatorname{ar} \bar{\mu} \sin \left(\frac{1}{\bar{\mu}} c\right) \cos \left(\frac{1}{\bar{\mu}} c\right)
$$

By using $p_{\text {repl }}=a^{2}$ as a substitution, the evolution of scale factor with time can be obtained:

$$
\dot{a}=\gamma \bar{\mu} \sin \left(\frac{1}{\bar{\mu}} c\right) \cos \left(\frac{1}{\bar{\mu}} c\right)
$$

On the other hand, by the Hamiltonian constraint $H_{Q, \text { repl }} \approx 0$, we can get:

$$
\sin ^{2}\left(\frac{1}{\bar{\mu}} c\right)=\frac{8 \pi a}{3 G \gamma^{2} \bar{\mu}^{2}} H_{E}
$$

By combining the above two formulas, the modified non Friedmann equation related to repulsion is obtained:

$$
H_{r e p l}^{2}=\frac{8 \pi}{3 G} \sigma\left(1-\frac{\sigma}{\sigma_{c}}\right)
$$

where the critical density is

$$
\sigma_{c}=\frac{\sqrt{3} \gamma^{3}}{16 \pi^{2}} \sigma_{p}, \quad \sigma_{p}=G^{2} \text { is the first density of Planck. }
$$

The appearance of the critical density $\sigma_{c}$ is completely a quantum repulsive effect. It makes the repulsive energy density $\sigma$ of the universe no longer diverge in any case, so the curvature of space-time 
is no longer diverging. When the quantum repulsion effect can be ignored, that is $\hbar \rightarrow 0$, then $\sigma_{c} \rightarrow \infty$, this goes back to the classical case. In particular, in the very early universe, $\sigma=\sigma_{c}$ was a rebound point, which replaced the singularity of the big bang in the classical theory.

( 5.3 ) the hedge-unified expansive universe equation related to the grand unification of loop quantum gravity and loop quantum repulsion

On the basis of the above discussion, for the classical flat FRW universe (Friedmann-Robertson -Walker), the Hamiltonian constraint of the grand unification of gravity and repulsion can be simply expressed as

$$
H_{c l, r e p l}-H_{c l, g r a v}=-\frac{3 G}{8 \pi} \gamma^{2} \sqrt{p_{\text {repl }}} c^{2}+H_{E}+\frac{3}{8 \pi G \gamma^{2}} \sqrt{p_{\text {grav }}} c^{2}-H_{M}
$$

where, the momentum of repulsion $p_{\text {repl }}=a^{2}, c=\gamma \dot{a}, \gamma \approx 0.2375, a$ is the scale factor, and $H_{E}$ is the Hamiltonian of the repulsive energy, the momentum of gravity $p_{\text {grav }}=1 / a^{2}, c=\gamma \dot{a}, \quad \gamma \approx 0.2375$, $a$ is the scale factor, and $H_{M}$ is the Hamiltonian of the gravitational mass.

If $H_{E}-H_{M} \approx 0$, the hedge-unified expansive universe equation related to the interaction of gravity and repulsion can be obtained in the case of $k=0$ (in the FRW scale, $k=0$ corresponds to a flat universe):

$$
\dot{H}_{r e p l}-\dot{H}_{\text {grav }}=-\frac{4 \pi}{G}\left(p_{\text {repl }}+\sigma\right)+k a^{2}+4 \pi G(p+\rho)-\frac{k}{a^{2}}
$$

here, when $a \rightarrow 0$, the energy density is divergent, which is the singularity moment of the big bang. When the loop quantum repulsion effect and the loop quantum gravitational effect cannot be ignored, the Hamiltonian constraint of the original hedge between the repulsion and the gravitation is modified as follows:

$$
H_{Q, \text { repl }}-H_{Q, \text { grav }}=-\frac{3 G \bar{\mu}^{2}}{8 \pi} \gamma^{2} \sqrt{p_{\text {repl }}} \sin ^{2}\left(\frac{1}{\bar{\mu}} c\right)+H_{E}+\frac{3}{8 \pi G \gamma^{2} \bar{\mu}^{2}} \sqrt{p} \sin ^{2}(\bar{\mu} c)-H_{M}
$$

where $\bar{\mu}$ is the length of the loop that makes up the smallest facet.

$$
\text { When } \frac{1}{\bar{\mu}}-\bar{\mu} \rightarrow 0 \text {, there is } \bar{\mu}^{2} \sin ^{2}\left(\frac{1}{\bar{\mu}} c\right)-\frac{1}{\bar{\mu}^{2}} \sin ^{2}(\bar{\mu} c) \rightarrow 1 \text {, }
$$

then $H_{Q, \text { repl }}-H_{Q, \text { grav }} \rightarrow H_{c l, h e d g e}$ will return to the classical limit. Through the Hamiltonian equation related to the hedge between the quantum repulsion and the quantum gravitation:

$$
\begin{aligned}
\dot{p}_{\text {hedge }} & =\left\{p_{\text {hedge }}, H_{Q, \text { hedge }}\right\} \\
& =-\frac{8 \pi}{3 G \gamma} \frac{\partial H_{Q, \text { repl }}}{\partial c}+\frac{8 \pi G \gamma}{3} \frac{\partial H_{Q, \text { grav }}}{\partial c}
\end{aligned}
$$




$$
=2 a \gamma \bar{\mu} \sin \left(\frac{1}{\bar{\mu}} c\right) \cos \left(\frac{1}{\bar{\mu}} c\right)-\frac{2 a}{\gamma \bar{\mu}} \sin (\bar{\mu} c) \cos (\bar{\mu} c)
$$

By using $p_{\text {repl }}=a^{2}$ and $p_{\text {grav }}=1 / a^{2}$ as the substitution, the hedge evolution of scale factor with time can be obtained:

$$
\dot{a}_{\text {hedge }}=\gamma \bar{\mu} \sin \left(\frac{1}{\bar{\mu}} c\right) \cos \left(\frac{1}{\bar{\mu}} c\right)-\frac{1}{\gamma \bar{\mu}} \sin (\bar{\mu} c) \cos (\bar{\mu} c)
$$

On the other hand, by the Hamiltonian constraint $H_{Q, \text { grav }}-H_{Q, \text { repl }} \approx 0$ of the hedge action, we can get:

$$
\sin ^{2}\left(\frac{1}{\bar{\mu}} c\right)-\sin ^{2}(\bar{\mu} c)=\frac{8 \pi a}{3 G \gamma^{2} \bar{\mu}^{2}} H_{E}-\frac{8 \pi G \gamma^{2} \bar{\mu}^{2}}{3 a} H_{M}
$$

By combining the above two formulas, the modified quantum hedge-unified expansive universe equation related to the quantum repulsion and the quantum gravitation is obtained:

$$
H_{r e p l}^{2}-H_{\text {grav }}^{2}=\frac{8 \pi}{3 G} \sigma\left(1-\frac{\sigma}{\sigma_{c}}\right)-\frac{8 \pi G}{3} \rho\left(1-\frac{\rho}{\rho_{c}}\right)
$$

where the critical density is

$$
\begin{gathered}
\sigma_{c}-\rho_{c}=\frac{\sqrt{3} \gamma^{3}}{16 \pi^{2}} \sigma_{p}-\frac{\sqrt{3}}{16 \pi^{2} \gamma^{3}} \rho_{p}, \\
\sigma_{p}-\rho_{p}=G-\frac{1}{G^{2}} \text { is the first density of Planck. }
\end{gathered}
$$

The appearance of the critical density $\sigma_{c}$ of the hedge matter ( mass and energy ) is completely a quantum hedge effect.

Therefore, the semi-classical quantum hedg-unified cosmology shows us the evolution of the universe from contraction to rebound and then to expansion.

\section{Expansive Universe Equation beyond Friedman's Equation}

Under the big unified framework of gravitational field and repulsive field, this series of work comprehensively expands the special and general relativity, establishes the repulsive field equation against the gravitational field equation, and then establishes the hedge-unified field equation describing the interaction of gravitational field and repulsive field; On this basis, the non-Friedmann equation against the Friedmann equation is established, thus a set of new equations describing the expansive universe model under the condition of fully considering the hedge effect of gravitational field and repulsion field, which can be called the expansive universe equation of hedge-unified field theory, is established.

( 6.1 ) Friedmann equation related to gravitational field

As we know, Friedman's equation is a set of equations describing the homogeneous and isotropic expansive universe model in space under the framework of general relativity. In our opinion, this 
equation is an expansive universe model related to the gravitational field equation.

In this regard, consider the Robertson-Walker metric

$$
d s^{2}=d t^{2}-R^{2}(t)\left\{\frac{d r^{2}}{1-k r^{2}}+r^{2} d \theta^{2}+r^{2} \sin ^{2} \theta d \varphi^{2}\right\}
$$

where $r, \theta$ and $\varphi$ are polar coordinates of the sphere and $t$ is the cosmic time, three values of $+1,0$ and - 1 can be taken as the spatial curvature sign $k$, and the time function $R(t)$ is called the cosmic scale factor. For a homogeneous isotropic universe, the medium energy momentum tensor of a gravitational source is in the form of

$$
T_{\mu v}=(\rho+p) U_{\mu} U_{v}+p g_{\mu v}
$$

where $\rho$ is the mass density of gravitational source, $U_{\mu}$ and $U_{\nu}$ are the quantities related to the gravitational potential, $p$ is the pressure of gravitational source,

$$
T_{00}=\rho(t), T_{0 i}=T_{i 0}=0, T_{i j}=-g_{i j} p(t) \text {, indexes } i, j=1,2,3 .
$$

For Einstein's gravitational field equation:

$$
R_{\mu \nu}-\Lambda g_{\mu \nu}=-8 \pi G\left(T_{\mu \nu}-\frac{1}{2} g_{\mu v} T\right)
$$

By substituting (84) and (85) into (86), two component formulas of the gravitational field equation are obtained:

time-time component equation:

$$
\ddot{R}=-\frac{4 \pi G}{3}(\rho+3 p) R+\frac{\Lambda}{3} R
$$

space-space component equation:

$$
R \ddot{R}+2 \dot{R}^{2}+2 k=4 \pi G(\rho-p) R^{2}+\Lambda R^{2}
$$

From (87) and (88), we get:

$$
\dot{R}^{2}+k=\frac{8 \pi G}{3} \rho R^{2}+\frac{\Lambda}{3} R^{2}
$$

Change equation (89) to

$$
H^{2} \equiv\left(\frac{\dot{R}}{R}\right)^{2}=\frac{8 \pi G}{3} \rho+\frac{\Lambda}{3}-\frac{k}{R^{2}}
$$

This formula is known as Friedman's equation.

It shows that, in fact, the expansion of the universe is driven by three main terms: matter term, cosmological constant term and curvature term.

By satisfying the energy momentum tensor $T_{v}^{\mu \nu}=0$, we can get: 
where the non-zero connection is

$$
\frac{d}{d R}\left(\rho R^{3}\right)=-3 p R^{2}
$$

$$
\Gamma_{i j}^{t}=R \dot{R} \tilde{g}_{i j}, \quad \Gamma_{t j}^{i}=\frac{\dot{R}}{R} \delta_{j}^{i}, \quad \Gamma_{j k}^{i}=\frac{1}{2} g^{i l}\left(\tilde{g}_{l j, k}+\tilde{g}_{l k, j}-\tilde{g}_{j k, l}\right),
$$

Index in the formula: $i, j, k, l=1,2,3$, and there is $g_{i j}=-R^{2}(t) \tilde{g}_{i j}$.

Now, we establish the non Friedman equation corresponding to the Friedman equation.

( 6. 2 ) non Friedman equation

As an improvement and extension of Friedman's equation, the non Friedman's equation is a new set of equations describing the homogeneous and isotropic expansion universe model in space under the framework of repulsion field. In our view, the non Friedman equation is an expansion universe model related to the repulsion field equation.

Under the Robertson-Walker metric, for a homogeneous isotropic universe, the form of the medium energy momentum tensor of the repulsive source is

$$
K_{\mu \nu}=\left(\sigma+p_{\text {repl }}\right) V_{\mu} V_{v}+p_{\text {repl }} g_{\mu v}
$$

where $\sigma$ is the mass density of repulsive source, $V_{\mu}$ and $V_{\nu}$ are the quantities related to the repulsive potential, $p_{\text {repl }}$ is the pressure of repulsive source,

$$
K_{00}=\sigma(t), K_{0 i}=K_{i 0}=0, K_{i j}=g_{i j} p_{\text {repl }}(t), \text { indexes } i, j=1,2,3 .
$$

Under the new framework, the following form of repulsion field equation can be established:

$$
\frac{1}{R_{\mu \nu}}+\Lambda g_{\mu \nu}=-\frac{8 \pi}{G}\left(K_{\mu \nu}-\frac{1}{2} g_{\mu \nu} K\right)
$$

By substituting (84) and (93) into (94), two component formulas of the repulsive field equation are obtained:

time-time component equation:

$$
\frac{1}{\ddot{R}}=-\frac{4 \pi}{3 G R}\left(\sigma+3 p_{\text {repl }}\right)-\frac{\Lambda}{3 R}
$$

space-space component equation:

$$
\frac{1}{R \ddot{R}}+\frac{2}{\dot{R}^{2}}-2 k=\frac{1}{G R^{2}} 4 \pi\left(\sigma-p_{\text {repl }}\right)-\frac{\Lambda}{R^{2}}
$$

From (95) and (96), we get:

$$
\frac{1}{\dot{R}^{2}}-k=\frac{8 \pi}{3 G R^{2}} \sigma-\frac{1}{3 R^{2}} \Lambda
$$

Rewrite formula (97) to 


$$
H_{\text {repl }}^{2} \equiv\left(\frac{R}{\dot{R}}\right)^{2}=\frac{8 \pi}{3 G} \sigma-\frac{\Lambda}{3}+k R^{2}
$$

This equation can be called non Friedman's equation.

It shows that, in fact, the expansion of the universe is driven by three main terms: repulsive force term, energy term and curvature term.

By satisfying the energy momentum tensor $K_{v}^{\mu v}=0$ of repulsive field we can get:

$$
\frac{d}{d R}\left(\frac{1}{R^{3}} \sigma\right)=\frac{1}{R^{2}} 3 p_{\text {repl }}
$$

where the non-zero connection is

$$
\Xi_{i j}^{t}=\frac{1}{R \dot{R}} \bar{g}_{i j}, \quad \Xi_{t j}^{i}=\frac{R}{\dot{R}} \delta_{j}^{i}, \quad \Xi_{j k}^{i}=\frac{1}{2} g^{i l}\left(\bar{g}_{l j, k}+\bar{g}_{l k, j}-\bar{g}_{j k, l}\right),
$$

The indexes in the formula: $i, j, k, l=1,2,3$, and there is $g_{i j}=-\frac{1}{R^{2}(t)} \bar{g}_{i j}$.

On the basis of the above discussion, we can combine the Friedman equation with the non Friedman equation, to establish a new equation called the hedge-unified expansive universe equation.

( 6.3 ) hedge-unified expansive universe equation

As a systematic combination of Friedman's equation and non Friedman's equation, the expansive universe equation of hedge-unified field theory is a new way to describe the homogeneous and isotropic expansion universe model in space under the framework of the grand unification of gravitational field and repulsive field.

Under the Robertson-Walker metric, for a homogeneous isotropic universe, the form of the medium energy momentum tensor of the hedge-unified medium is

$$
K_{\mu \nu}-T_{\mu v}=\left(\sigma+p_{\text {repl }}\right) V_{\mu} V_{v}+p_{\text {repl }} g_{\mu \nu}-\left(\rho+p_{\text {grav }}\right) U_{\mu} U_{v}-p_{\text {grav }} g_{\mu v}
$$

where $\sigma$ is the mass density of repulsive source, $V_{\mu}$ and $V_{\nu}$ are the quantities related to the repulsive potential, $p_{\text {repl }}$ is the pressure of repulsive source, $\rho$ is the mass density of gravitational source, $U_{\mu}$ and $U_{v}$ are the quantities related to the gravitational potential, $p$ is the pressure of gravitational source,

$$
\begin{aligned}
& K_{00}-T_{00}=\sigma(t)-\rho(t), \\
& K_{0 i}-T_{0 i}=K_{i 0}-T_{i 0}=0, \\
& K_{i j}-T_{i j}=-g_{i j} p_{\text {repl }}(t)-g_{i j} p_{\text {grav }}(t)=-g_{i j}\left[p_{\text {repl }}(t)+p_{\text {grav }}(t)\right], \text { indesxes } i, j=1,2,3 .
\end{aligned}
$$

Now, we can establish the hedge-unified field equation of which combines the gravitational field equation with the repulsive field equation:

$$
\frac{1}{R_{\mu \nu}}-R_{\mu \nu}+2 \Lambda g_{\mu \nu}=-\frac{8 \pi}{G}\left(K_{\mu \nu}-\frac{1}{2} g_{\mu v} K\right)+8 \pi G\left(T_{\mu \nu}-\frac{1}{2} g_{\mu \nu} T\right)
$$


By substituting (84) and (101) into (102), two component formulas of the hedge-unified field equation are obtained:

time-time component equation:

$$
\frac{1}{\ddot{R}}-\ddot{R}=-\frac{4 \pi}{3 G R}\left(\sigma+3 p_{\text {repl }}\right) R-\frac{\Lambda}{3} R+\frac{4 \pi G}{3}\left(\rho+3 p_{\text {grav }}\right) R-\frac{\Lambda}{3} R
$$

space-space component equation:

$$
\frac{1}{R \ddot{R}}+\frac{2}{\dot{R}^{2}}-R \ddot{R}-2 \dot{R}^{2}-4 k=\frac{1}{G R^{2}} 4 \pi\left(\sigma-p_{\text {repl }}\right)-\frac{\Lambda}{R^{2}}-4 \pi G\left(\rho-p_{\text {grav }}\right) R^{2}-\Lambda R^{2}
$$

From (103) and (104), we get:

$$
\frac{1}{\dot{R}^{2}}+\dot{R}^{2}-2 k=\frac{8 \pi G}{3 G R^{2}} \sigma-\frac{1}{3 R^{2}} \Lambda-\frac{8 \pi G}{3} \rho R^{2}-\frac{\Lambda}{3} R^{2}
$$

Rewrite formula (105) to

$$
\begin{aligned}
H_{\text {repl }}^{2}-H_{\text {grav }}^{2} & \equiv\left(\frac{R}{\dot{R}}\right)^{2}-\left(\frac{\dot{R}}{R}\right)^{2} \\
& =\frac{8 \pi}{3 G} \sigma-\frac{\Lambda}{3}+k R^{2}-\frac{8 \pi G}{3} \rho-\frac{\Lambda}{3}+\frac{k}{R^{2}}
\end{aligned}
$$

This equation can be called the expansive universe equation of hedge-unified field theory.

It shows that, in fact, the expansion of the universe is driven by three main terms: repulsive force term, gravitation term and energy-momentum distribution term.

By satisfying the energy momentum tensor $K_{v}^{\mu v}-T_{v}^{\mu v}=0$ of hedge-unified field we can get:

$$
\frac{d}{d R}\left(\frac{1}{R^{3}} \sigma\right)-\frac{d}{d R}\left(\rho R^{3}\right)=-3\left(p_{\text {repl }} \frac{1}{R^{2}}-p_{\text {grav }} R^{2}\right)
$$

where the non-zero connection is

$$
\begin{aligned}
& \Xi_{i j}^{t}-\Gamma_{i j}^{t}=\frac{1}{R \dot{R}} \bar{g}_{i j}-R \dot{R} \tilde{g}_{i j}, \\
& \Xi_{t j}^{i}-\Gamma_{t j}^{i}=\delta_{j}^{i}\left(\frac{R}{\dot{R}}-\frac{\dot{R}}{R}\right), \\
& \Xi_{j k}^{i}-\Gamma_{j k}^{i}=\frac{1}{2} g^{i l}\left(\bar{g}_{l j, k}+\bar{g}_{l k, j}-\bar{g}_{j k, l}\right)-\frac{1}{2} g^{i l}\left(\tilde{g}_{l j, k}+\tilde{g}_{l k, j}-\tilde{g}_{j k, l}\right),
\end{aligned}
$$

The indexex $i, j, k, l=1,2,3$, and there is

$$
g_{i j}=-\frac{1}{R^{2}(t)} \bar{g}_{i j}+R^{2}(t) \tilde{g}_{i j}
$$




\section{Hyperholographic Quantum Correspondence}

Now, at the level of quantum gravity and quantum repulsion, we explore and propose a holographic quantum unifying interaction mechanism that can be promoted to the basic principle. This mechanism discussed in this paper is derived from the holographic principle and holographic dark energy model, and the holographic principle is closely related to the entropy of black hole.

J. D. Beckenstein introduced black hole entropy when he extended the second law of thermodynamics to the second law of generalized thermodynamics. The entropy expression of black hole calculated by quantum field theory is: $S_{B H}=\frac{k c^{3}}{4 \hbar G} A$ where $A$ is the area of event horizon of black hole, $\hbar$ is the reduced Planck constant, $\kappa$ is the Boltzmann constant, $G$ is the Newton gravitational constant, and $c$ is the speed of vacuum light. In the natural system of units, the reduced Planck constant $\hbar$ (i.e. $\left(\frac{h}{2 \pi}\right)$ ), light speed constant $c$, Boltzmann constant $\kappa$ are all equal to 1 . The expression of black hole entropy can be written as: $S_{B H}=\pi r_{H}^{2} M_{p}^{2}$, where $r_{H}$ is the radius of black hole, $M_{p}$ is Planck mass. G. 't Hooft proposed the holographic principle in 1993 ${ }^{[93]}$, and L. Suskind further elaborated the holographic principle in $1994^{[94]}$. The duality relationship between infrared truncation and ultraviolet truncation limits the research scope of holographic principle.

It is worth noting that no matter what we choose infrared truncation and ultraviolet truncation, these two scales always depend on the evolution of the universe ${ }^{[95]-[97]}$, and the mechanism of holographic unifying action can be used as the basis of the evolution of the universe. Further, we can give the model of quantum repulsion enhancement as infrared truncation, and take the holographic quantum unifying action mechanism as the basis of the coupling of dark matter and dark energy, as shown in Figure 2 (Li Zongcheng, 2019).

It has been known that loop quantum gravity (LQG) is a non perturbative regular theory of quantum gravity. In this theory, the description of space-time is background independent, and its geometry is composed of spin network. In 2005, M. Bojowald and others applied some important achievements of LQG to the research of cosmology, thus forming a set of semiclassical loop quantum cosmology theory ${ }^{[98]-[100]}$. It is believed that this theory can not only deduce a big rebound at the beginning of the expansion of the universe, so as to avoid the singularity of the big bang, but also help to explain the accelerating expansion of the universe and the final result of the universe.

Among many dark matter candidates, Elko field has a very good description of the strange properties of dark matter, and is regarded as one of the important candidates of dark matter ${ }^{[101][102]}$. On the other hand, the membrane world theory, which can solve the problems of hierarchy, cosmological constants and other physical problems, has attracted widespread attention ${ }^{[69][103][104]}$. It is very important to study the localization mechanism of matter field in the membrane world. Through localization, we can reconstruct the standard model of field theory on membrane and provide guidance for future experiments. At the same time, for the matter field such as Elko field, the dark matter model is facing 
the challenge of black hole "hairless" theorem, which rejects the stable existence of other matter fields outside the black hole except electromagnetic field. However, the theorem does not reject the fact that the matter field has evolved outside the black hole for a long time. Recently, a new configuration of matter field, quasi bound state, has been proposed, which can exist outside black hole for a long time. Its evolution time is likely to reach the age of the universe. As soon as this new field configuration appears, people are interested in it.

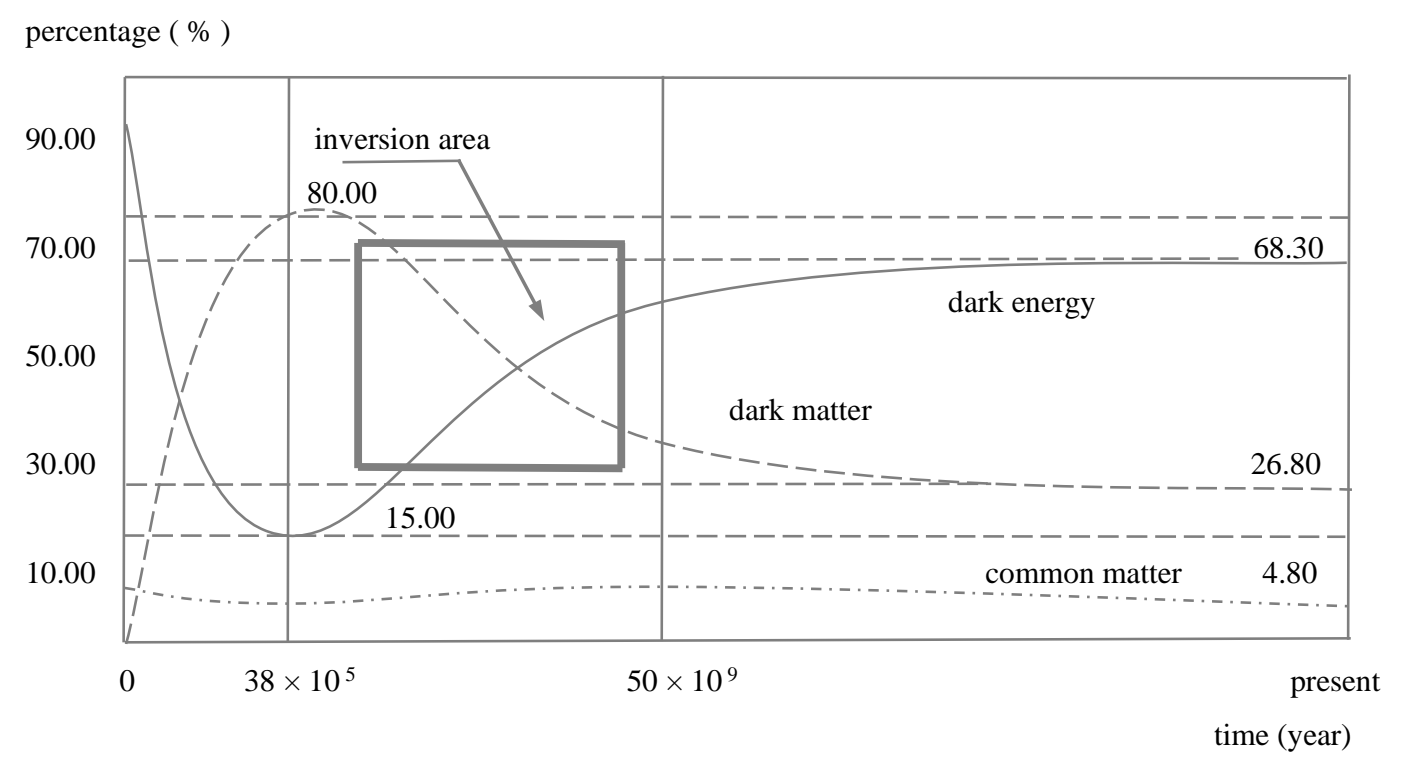

Fig. 2 Changes of Distribution Percentage of Dark Energy, Dark Matter and Conventional Matter during Evolution of Universe

It has been noted that ${ }^{[105]}$ for the 5-D free and massless Elko field, the zero mode and the KK mode with mass can not be localized in the flat membrane world, either in the thin membrane model or in the thick membrane model. For Elko field with 5-D coupling term, its zero mode can be localized on RSII model only when the 5-D mass is negative. If we introduce Yukawa-like coupling term, for some special thick membrane models, and the coupling constant takes a special value, its zero mode can be localized on these special thick membranes. However, for the KK mode with mass, it can not be localized on the thin membrane and thick membrane models. In addition, we also consider the localization properties of the zero mode of 5-D free zero mass Elko field on the De Sitter / anti de Sitter membranes. It is found that the zero mode can not be localized on the De Sitter / anti de Sitter membranes. It can be seen that the localization of Elko field is challenging, and we need to consider more models and coupling methods.

In addition, the high-dimensional cosmological model and dark energy problem are discussed in [106]. In this model, the universe is a hypersurface embedded in 5-dimensional Ricci flat space-time. In five dimensions, it's empty or vacuum. However, from the perspective of 4-dimensions, the universe is full of matter induced from extra dimensions, which is called induced matter. There is a family of exact five dimensional vacuum solutions which contain two functions $\mu(t)$ and $v(t)$ about time $t$. By choosing this function properly, the rebound singularity of the rebound cosmology model described by this solution is studied. The results show that at the rebound point, the topological invariants and energy 
density of the three 5-dimensions are limited. However, the pressure experienced a sharp transformation from negative infinity to positive infinity. Therefore, the singularity is a material singularity, not a spatiotemporal singularity. The results show that the scalar potential in quintessence corresponds to the special $f(z)$ form in our model. Given this correspondence, we can get $f(z)$, which makes selection easier and has a stronger physical basis. In addition, from the point of view of model independence, the state equation $w(z)$ and the relationship between the deceleration factor $Q(z)$ and $f(z)$ are established. Therefore, under the guidance of the observation data, we get the specific form of function $f(z)$. At this time, the 5-dimensional cosmological model is rebuilt.

Literature [107] mainly studies the 5-dimensional STM theory and the membrane world model in the high-dimensional cosmology model. It uses energy conditions to study the induced substance, which corresponds to the different changes of energy conditions and reflects the changes of material components. Next, the energy condition is used to distinguish different membrane models, and it is used to analyze the material composition of DGP membrane. Then the rebound solution of 5-D vacuum is equal to the solution of DGP membrane model given by Dick, and the specific geometric meaning of any constant $I$ in the DGP model is given. At the same time, the relation between any function $\mu$ and $v$ in the rebound solution is given. Later, considering the DGP model with Tachyon field on the membrane, it is found that the Friedmann equation with Tachyon field can be embedded on the membrane under certain conditions, and the scale form of 5-dimensional space-time is given, and the power-law solution of the universe is also obtained. Finally, the global membrane world model is studied, in which the Friedmann equation is modified by the term of any function $v$. This term is defined as the dark energy density, which will accelerate the expansion of the universe and affect the evolution of the universe.

Now, we give a new mechanism explanation by expanding the theory of loop quantum cosmology.

Considering the interaction between matter and dark energy, each of $\rho_{\text {grav }}$ and $\rho_{\text {repl }}$ no longer meet the energy conservation equation alone, but should meet the balance equation:

$$
\dot{\rho}_{\text {grav }}+3 H \rho_{\text {grav }}=-Q, \quad \dot{\rho}_{\text {repl }}+3 H\left(1+w_{\text {repl }}\right) \rho_{\text {repl }}=Q,
$$

where $Q$ is the quantum unifying action term of the interaction between quantum gravity and quantum repulsion. Here, it is assumed that the quantum unifying action is directly proportional to the total energy density of the universe, i.e., the form is taken as $Q=3 c H\left(\rho_{\text {grav }}+\rho_{\text {repl }}\right)$, in which $c$ is a dimensionless constant. With the expression of dimensionless density, we can obtain the constraint :

$$
\Omega_{\text {grav }}+\Omega_{\text {repl }}=1
$$

and the dimensionless density parameters: $\Omega_{\text {grav }}=\rho_{\text {grav }} / \rho_{\text {grav }, c}, \Omega_{\text {repl }}=\rho_{\text {repl }} / \rho_{\text {repl }, c}$.

In addition, substituting the dimensionless density expression into equation ( 109 ), we can get:

$2 \frac{\dot{H}}{H^{2}}\left(1-\Omega_{\text {repl }}\right)-\frac{\dot{\Omega}_{\text {repl }}}{H}+3\left(1-\Omega_{\text {repl }}\right)+3 c=2 \frac{\dot{H}}{H^{2}}\left(1-\Omega_{\Lambda}\right)-\frac{\dot{\Omega}_{\Lambda}}{H}+3\left(1-\Omega_{\Lambda}\right)+3 c=0$.

The Hubble scale $H$ is chosen as the infrared truncation scale, and the 5-dimensional quantum 
universe is chosen as the energy truncation scale. The density of holographic dark energy is $\rho_{\text {repl }}=\rho_{A}=$ $B H, \rho_{A}$ is the dark energy density related to the cosmological constant $\Lambda$, and $B$ is the constant.

By substituting this equation into equation ( 109 ) and deriving the two sides of the equation, we get:

$$
\frac{\dot{H}}{H^{2}}=-\frac{3 M_{p}^{2}}{B} \dot{\Omega}_{r e p l}=-\frac{3 M_{p}^{2}}{B} \dot{\Omega}_{\Lambda}, \quad \dot{\Omega}_{\text {repl }}=\Omega_{\text {repl }}^{\prime} H=\Omega_{\Lambda}^{\prime} H,
$$

where $\Omega_{r e p l}^{\prime} \equiv \frac{d \Omega_{r e p l}}{d \ln a}=\frac{d \Omega_{\Lambda}}{d \ln a}, \Omega_{\Lambda}=\rho_{\Lambda} / \rho_{\Lambda, c}$, therefore

$$
\frac{\dot{H}}{H^{2}}=-\frac{\Omega_{r e p l}^{\prime}}{\Omega_{r e p l}}=-\frac{\Omega_{\Lambda}^{\prime}}{\Omega_{\Lambda}}
$$

Substituting equation ( 112 ) into equation ( 111 ), we can get:

$$
\left(\frac{\Omega_{\text {repl }}-2}{\Omega_{\text {repl }}}\right) \Omega_{\text {repl }}^{\prime}+3\left(1-\Omega_{\text {repl }}\right)+3 c=\left(\frac{\Omega_{\Lambda}-2}{\Omega_{\Lambda}}\right) \Omega_{\Lambda}^{\prime}+3\left(1-\Omega_{\Lambda}\right)+3 c=0 .
$$

In addition, by equation ( 109 ), we get:

$$
\begin{gathered}
\dot{\rho}_{\text {repl }}+3 H\left(1+w^{e f f}\right)=\dot{\rho}_{\Lambda}+3 H\left(1+w^{e f f}\right)=0, \\
w^{e f f}=w_{\text {repl }}+c\left(1+\frac{\rho_{\text {grav }}}{\rho_{\text {repl }}}\right)=w_{\Lambda}+c\left(1+\frac{\rho_{\text {grav }}}{\rho_{\Lambda}}\right) .
\end{gathered}
$$

Therefore, $w^{\text {eff }}$ can be expressed as:

$$
w^{e f f}=-1-\frac{\dot{\rho}_{\text {repl }}}{3 H \rho_{\text {repl }}}=-1+\frac{1}{3} \frac{\Omega_{\text {repl }}^{\prime}}{\Omega_{\text {repl }}}=-1+\frac{1}{3} \frac{\Omega_{\Lambda}^{\prime}}{\Omega_{\Lambda}} .
$$

In order to investigate the early inflation and the present accelerating expansion of the universe, we also need to calculate the deceleration factor $q$. By equation ( 112 ), we can get:

$$
q=-\frac{\dot{H}}{H^{2}}-1=-1+\frac{\Omega_{r e p l}^{\prime}}{\Omega_{r e p l}}=-1+\frac{\Omega_{\Lambda}^{\prime}}{\Omega_{\Lambda}} .
$$

Because of

$$
\Omega_{\Lambda}^{\prime}=\frac{d \Omega_{\Lambda}}{d \ln a}=-(1+z) \frac{d \Omega_{\Lambda}}{d z},
$$

where $z=\frac{1}{a}-1$, formula ( 113 ), formula ( 114 ) and formula ( 115 ) can be rewritten as:

$$
(1+z) \frac{2-\Omega_{\Lambda}}{\Omega_{\Lambda}} \frac{d \Omega_{\Lambda}}{d z} \frac{1}{\Omega_{\Lambda}}+3\left(1-\Omega_{\Lambda}\right)+3 c=0
$$




$$
\begin{gathered}
w^{e f f}=-1-(1+z) \frac{1}{3} \frac{d \Omega_{\Lambda}}{d z} \frac{1}{\Omega_{\Lambda}} \\
q=-1-(1+z) \frac{d \Omega_{\Lambda}}{d z} \frac{1}{\Omega_{\Lambda}}
\end{gathered}
$$

It can be seen from equation ( 117 ) that in the future, the value of the effective state parameter will approach to -1 , but it will not exceed -1 . This shows that there will be no future of big tears in the universe. Even in the infinite future, $z \rightarrow-1$, the first term of formula ( 117 ) tends to zero.

As long as it is recognized that the dark energy component is still increasing at present $(z=0)$, the value of $\Omega_{A}$ will always be less than $1+c$. Therefore, from equation ( 117 ) and equation ( 118 ), we can see that the value of $w^{\text {eff }}$ is always greater than and tends to -1 .

Therefore, we propose a basic inference that can be promoted to the hyperholographic synergy principle of high dimensional universe.

The interaction between supergravity and superrepulsion in the complex evolution of the high-dimensional universe forms a dual relationship with the interaction between quantum gravity and quantum repulsion in the early inflation of the 4-dimensional universe.

The interaction between quantum gravity and quantum repulsion in the early inflation of the 4-dimensional universe form a dual relationship with the interaction between quantum-classical gravity and quantum-classical repulsion in the late accelerating expansion of the 4-dimensional universe.

Further, we propose a basic inference that can be promoted to the principle of hyperholography of high-dimensional universe (or multiuniverse): in a parallel universe formed by mutual coupling and holographic correspondence between the $P$ universe dominated by negative pressure (high-dimensional superrepulsion and quantum repulsion of accumulating positive energy, mainly composed of dark energy) and the $N$ universe dominated by positive pressure (high-dimensional supergravity and quantum gravity of accumulating negative energy, mainly composed of dark matter), there is a correspondence between the small-scale physics (ultraviolet cutoff) of the $P$ universe and the large-scale physics (infrared cutoff) of the $N$ universe, while there is a correspondence between the large-scale physics (ultraviolet cutoff) of the $P$ universe and the small-scale physics (infrared cutoff) of the $N$ universe.

The future event horizon as infrared truncation has been widely used in the literature ${ }^{[108][109]}$. Although choosing future event horizon as infrared truncation will lead to the problem of causality, it can alleviate the problem of coincidence. Now, we select future event horizon as infrared truncation, and 5-D black hole as energy truncation, then

$$
\rho_{\Lambda}=B\left(a \int_{a}^{\infty} \frac{d a}{H a^{2}}\right)^{-1}, \text { equivalently, } \frac{B}{\rho_{\Lambda} a}=\int_{a}^{\infty} \frac{d a}{H a^{2}} .
$$

To find the derivative of $a$ on both sides of the equal sign of the above formula at the same time, we can get

$$
\frac{B}{\rho_{\Lambda}^{2} a^{2}} \rho_{\Lambda}^{\prime}+\frac{B}{\rho_{\Lambda} a^{2}}=\frac{1}{H a^{2}}, \quad \rho_{\Lambda}^{\prime}=\frac{d \rho_{\Lambda}}{d \ln a}
$$


namely

$$
\frac{\Omega_{\Lambda}^{\prime}}{\Omega_{\Lambda}}+\frac{2 H^{\prime}}{H}+1-\frac{H}{H_{0}} b \Omega_{0}=0, \quad b \equiv \frac{3 M_{p}^{2} H_{0}}{B} .
$$

By introducing dimensionless variables $X \equiv \ln \frac{H}{H_{0}}$, substituting them into equation (111) and equation (122) and simplifying them, a set of dynamic equations for the evolution of the universe can be obtained as follows:

$$
\begin{gathered}
X^{\prime}=\frac{1}{2}\left[2 \Omega_{\Lambda}+b \Omega_{\Lambda}^{2} e^{X}-3(1+c)\right], \\
\Omega_{\Lambda}^{\prime}=b \Omega_{\Lambda}^{2} e^{X}-\Omega_{\Lambda}-\left[2 \Omega_{\Lambda}+b \Omega_{\Lambda}^{2} e^{X}-3(1+c)\right] \Omega_{\Lambda} .
\end{gathered}
$$

By a simple qualitative analysis of the above differential equations, we can see that this system of equations has a unique singularity:

$$
\left(\Omega_{\Lambda_{c}}, X_{c}\right)=\left(1+c, \ln \frac{1}{b(1+c)}\right) .
$$

To expand the system of equations to infinitesimal near the singular point, and consider only the first order infinitesimal term, we can get the following linear system:

$$
\begin{gathered}
\qquad X^{\prime}=\frac{1+c}{2} \delta X+2 \delta \Omega_{\Lambda}, \\
\qquad \Omega_{\Lambda}^{\prime}=(5+4 c) \delta X+(1+c)(2+c) \delta \Omega_{\Lambda} . \\
\text { The determinant of the coefficient matrix } A=\left(\begin{array}{cc}
\frac{1+c}{2} & 2 \\
(1+c)(2+c) & 5+4 c
\end{array}\right) \text { of the above plane linear }
\end{gathered}
$$

system is a

$$
\frac{1+c}{2}(5+4 c)-(1+c)(2+c) \delta \Omega_{\Lambda}=-\frac{3(1+c)}{2} .
$$

Based on the above, we now make the following inference:

(a1) the total amount of positive energy with strong negative pressure distribution in high-dimen space-time is constant, although this energy can flow between different dimensions of space-time; $(a 2)$ the total amount of negative energy with positive pressure distribution in high-dimen space-time is constant, although this energy can flow between different dimensions of space-time; (a3) the total amount of positive energy with strong negative pressure distribution in high-dimen space-time is equal to the total amount of negative energy distribution with positive pressure in higher dimen space-time.

Further inferences are made as follows: 
(b1) the total effective potential of repulsion supported by positive energy is constant in high-dim space-time, although the effective potential can be transformed in different dimensions of space-time; (b2) the total effective potential of gravity supported by negative energy is constant in high-dim space-time, although the effective potential can be transformed in different dimensions of space-time; (b3) the total effective potential of repulsion supported by positive energy in high-dim space-time is equal to the total effective potential of gravity supported by negative energy in high-dim space-time.

From the perspective of the whole universe, before the big bang, the high-dimen super-gravity field (collapse field) and the gravitational field (contraction field) dominate; after the big bang, the high-dimen superrepulsion field (inflation field) and the repulsion field (expansion field) dominate.

In terms of the basic components, before or after the big bang, the high-dimen hypergravitons and high-dimen hyperrepulsons are all coupled with each other, while the negative energy contraction dominated by the positive pressure and the positive energy expansion dominated by the negative pressure work alternately to form the arche-conjugate pulsator, and then form the quantum-conjugate pulsator.

It is necessary to establish the quantum-conjugate pulse dynamics and its super inflation universe model for the supersymmetry of 4-dimensional noncommutative space-time.

It is necessary to establish the arche-conjugate pulse dynamics and its high-dimensional quantum universe model for the supersymmetry of high-dimensional noncommutative space-time.

\section{Arche-Conjugation and Its Relation of Pulsation Mechanics}

For the initial universe at the level of quantum gravity and quantum repulsion, suppose the initial momentum $p=\Lambda$, where $\Lambda$ truncation is the energy scale of quantum unifying physics. We regard $H$ / $\Lambda$ as a free parameter, which is determined by experimental data ${ }^{[108][109]}$. The initial condition when the co moving momentum $k=a p=-\frac{\Lambda}{H k}$ can be written as follows:

$$
\eta_{0}=-\frac{\Lambda}{H k}
$$

The slow roll parameter $\eta=-\frac{1}{a H}$ is defined by scale $a$, and the FRW metric in the co moving coordinate is written as:

$$
d s^{2}=a \eta^{2}\left(-d \eta^{2}+d x^{2}\right)
$$

defining $\mu=a \varphi$, the oscillation formula of repulsive force can be written as follows:

$$
(\mu)^{\prime \prime}+\left(c_{3}^{2} k^{2}-\frac{a^{\prime \prime}}{a}\right) \mu=0 \text {. }
$$

It has been noted that in conformal time, whether power-law inflation $a(t) \propto t^{p}$ or index inflation $a(t) \propto e^{t}$, it can be written as power-law inflation $a(\eta) \propto \eta^{-c}$. When $c=1, w=-1$, the state is characterized by the cosmological constant. Therefore, the oscillation formula (127) of repulsive force 
can be written as follows:

$$
\mu^{\prime \prime}+\left[k^{2}-\frac{c(c+1)}{\eta^{2}}\right] \mu=0
$$

The solution of equation (128) should satisfy the asymptotic free condition:

$$
\lim _{k /(a H) \rightarrow \infty} \mu_{S, T}(\eta)=\frac{1}{\sqrt{2 k}} e^{-i k\left(\eta-\eta_{i}\right)} .
$$

To define conjugate momentum and its quantization:

$$
\begin{gathered}
\pi_{k}=\mu_{k}^{\prime}-\frac{a^{\prime}}{a} \mu_{k}, \\
\mu_{k}(\eta)=\frac{1}{\sqrt{2 k}}\left(a_{k}(\eta)+a_{-k}^{\dagger}(\eta)\right), \\
\pi_{k}(\eta)=-i \sqrt{\frac{k}{2}}\left(a_{k}(\eta)-a_{k}^{\dagger}(\eta)\right) .
\end{gathered}
$$

In the special conformal time $\eta_{0}$, by the Bogoliubov transformation, we have:

$$
\begin{aligned}
& a_{k}(\eta)=u_{k}(\eta) a_{k}\left(\eta_{0}\right)+v_{k}(\eta) a_{-k}^{\dagger}\left(\eta_{0}\right) \\
& a_{k}(\eta)=u_{k}(\eta) a_{-k}^{\dagger}\left(\eta_{0}\right)+v_{k}(\eta) a_{k}\left(\eta_{0}\right)
\end{aligned}
$$

So for $\mu_{k}$ and $\pi_{k}$, there should be

$$
\begin{gathered}
\mu_{k}(\eta)=f_{k}(\eta) a_{k}\left(\eta_{0}\right)+f_{k}^{*}(\eta) a^{\dagger}{ }_{-k}\left(\eta_{0}\right), \\
\pi_{k}(\eta)=-i\left(g_{k}(\eta) a_{k}\left(\eta_{0}\right)\right)+g_{k}^{*}(\eta) a_{-k}^{\dagger}\left(\eta_{0}\right) .
\end{gathered}
$$

where

$$
f_{k}(\eta)=\frac{1}{\sqrt{2 k}}\left(u_{k}(\eta)+v_{k}^{*}(\eta)\right), \quad g_{k}(\eta)=\sqrt{\frac{k}{2}}\left(u_{k}(\eta)-v_{k}^{*}(\eta)\right)
$$

For vacuum, select

$$
a_{k}\left(\eta_{0}\right) \mid 0, \eta_{0}>=0
$$

This means $v_{k}\left(\eta_{0}\right)=0, \pi_{k}\left(\eta_{0}\right)=i k \mu_{k}\left(\eta_{0}\right)$.

We combine the spherical harmonic function with its conjugate function together:

$$
f_{k}=\frac{A_{k}}{\sqrt{2 k}} e^{-i k \eta}\left(1-\frac{i}{k \eta}\right)+\frac{B_{k}}{\sqrt{2 k}} e^{i k \eta}\left(1+\frac{i}{k \eta}\right)
$$

as the solution of free function equation ( 129 ) at $c=1$.

Take $g_{k}=i \pi_{k}$ for $g_{k}$, and we can get the values of $u_{k}$ and $v_{k}$ by equation ( 134 ). According to 
Wronskian condition

$$
\mu_{k}^{*} \frac{d u_{k}}{d \tau}-u_{k} \frac{d u_{k}^{*}}{d \tau}=-i
$$

give $\left|A_{k}\right|^{2}-\left|B_{k}\right|^{2}=1$. The choice $v_{k}\left(\eta_{0}\right)=0$ of vacuum indicates that:

$$
B_{k}=\frac{1}{2 k \eta_{0}+i} i e^{-2 i k \eta_{0}} A_{k}
$$

Finally, the power spectrum of primary gravity can be written as follows:

$$
\begin{aligned}
P(k)=\frac{1}{2 \pi^{2} a^{2}} k^{3}\left|f_{k}\right|^{2} & \approx \frac{1}{4 \pi^{2} \eta^{2} a^{2}}\left(\left|A_{k}\right|^{2}+\left|B_{k}\right|^{2}-A_{k}^{*} B_{k}-A_{k} B_{k}^{*}\right) \\
& =\left(\frac{H}{2 \pi}\right)^{2}\left[1-\frac{H}{\Lambda} \sin \frac{2 \Lambda}{H}+\frac{H^{2}}{\Lambda^{2}} \sin \frac{\Lambda}{H}\right],
\end{aligned}
$$

This is the result of the gravitational tensor field, and a factor of $1 / \varepsilon$ should be added before the result of the repulsive scalar field. In general background time and space,

$$
u_{k}=\frac{1}{2} \sqrt{\frac{\pi}{k}} \sqrt{\frac{y}{1-\varepsilon}}\left[C_{+} H_{v}\left(\frac{y}{1-\varepsilon}\right)+C_{-} H_{v}^{*}\left(\frac{y}{1-\varepsilon}\right)\right],
$$

its result is

$$
P(k)=\left[1+\frac{H}{\Lambda} \sin \frac{2 \Lambda}{(1-\varepsilon) H}+\frac{H^{2}}{\Lambda^{2}} \sin \frac{\Lambda}{(1-\varepsilon) H}\right] P_{0}(k),
$$

here $P_{0}(k)$ is the power spectrum of BD vacuum.

There are many factors that affect the gravitational potential and repulsive potential. We mainly consider the field strength, mass density, energy density, repulsive potential (for gravity), gravitational potential (for repulsion), and so on.

The relationship between effective potential and mass density is shown in Figure 3 (Li Zongcheng, 2019); the relationship between effective potential and repulsive field strength is shown in Figure 4 (Li Zongcheng, 2019).

The relationship between effective potential and energy density is shown in Figure 5 ( $\mathrm{Li}$ Zongcheng, 2019); the relationship between effective potential and gravitational field strength is shown in Figure 6 (Li Zongcheng, 2019).

The quantum repulsion $S_{q-r e p l}=(E, p)$ with strong negative pressure and accumulating positive energy and the quantum gravity $S_{q-\text { grav }}=\left(-h v,-\frac{h}{\lambda} p_{0}\right)$ with positive pressure and accumulating negative energy constitute the arche-conjugate quantum unifying force: 


$$
S_{q-u n i t y}=\left((E,-h v),\left(p,-\frac{h}{\lambda} p_{0}\right)\right)
$$

where $E$ and $p$ represent the energy and momentum of the quantum system, and $v$ and $\lambda$ represent the frequency and wavelength of the quantum system,

$$
(E,-h v)=E+i(-h v),\left(p,-\frac{h}{\lambda} p_{0}\right)=p+i\left(-\frac{h}{\lambda} p_{0}\right)
$$

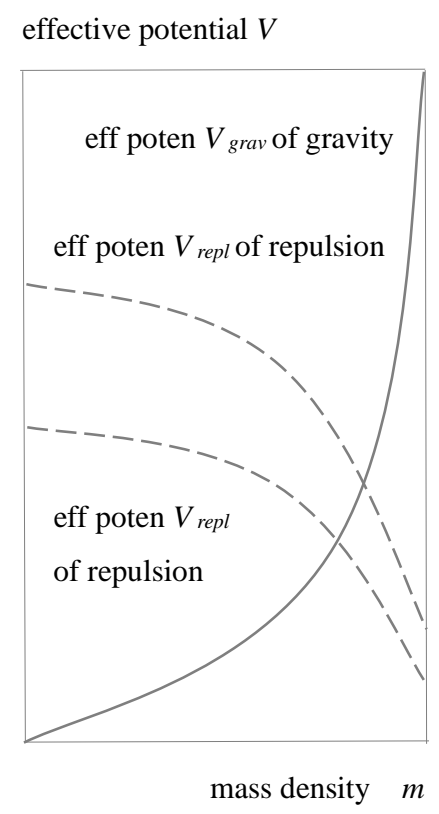

Fig 3 Eff Poten and Mass Density effective potential $V$

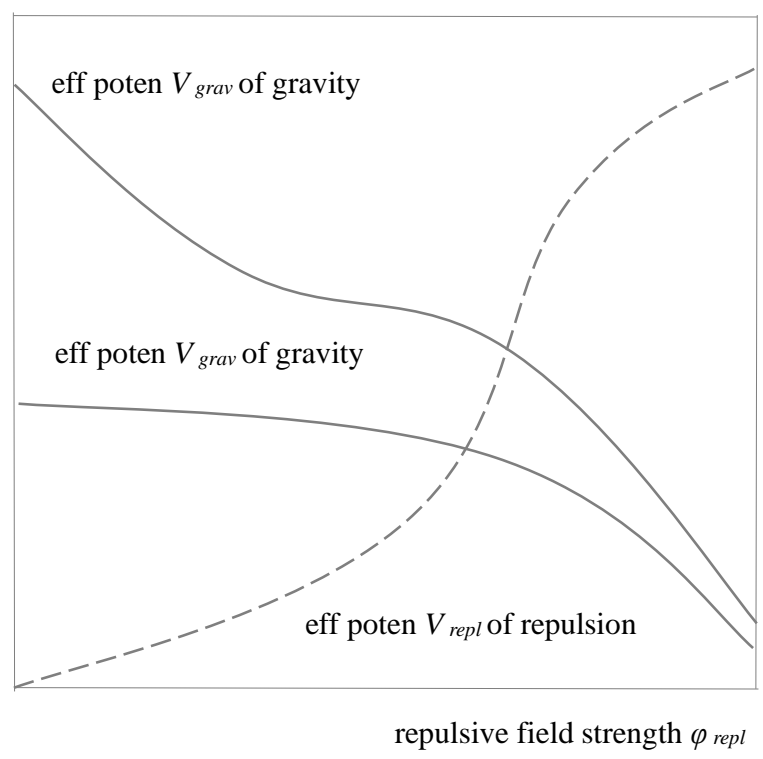

Fig 4 Eff Poten and Repulsive Field Strength

The quantum repulsion $S_{q-r e p l}=\left(h v, \frac{h}{\lambda} p_{0}\right)$ with strong negative pressure and accumulating positive energy and the quantum gravity $S_{q-\text { grav }}=(-E,-p)$ with positive pressure and accumulating negative energy constitute the arche-conjugate quantum unifying force:

$$
S_{q-u n i t y}=\left((h v,-E),\left(\frac{h}{\lambda} p_{0},-p\right)\right),
$$

where $E$ and $p$ represent the energy and momentum of the quantum system, and $v$ and $\lambda$ represent the frequency and wavelength of the quantum system,

$$
(h v,-E)=h v+i(-E),\left(\frac{h}{\lambda} p_{0},-p\right)=\left(-\frac{h}{\lambda} p_{0}\right)+i(-p)
$$

The arche-conjugate pulsation under the interaction of quantum repulsion and quantum gravity is as follows: 


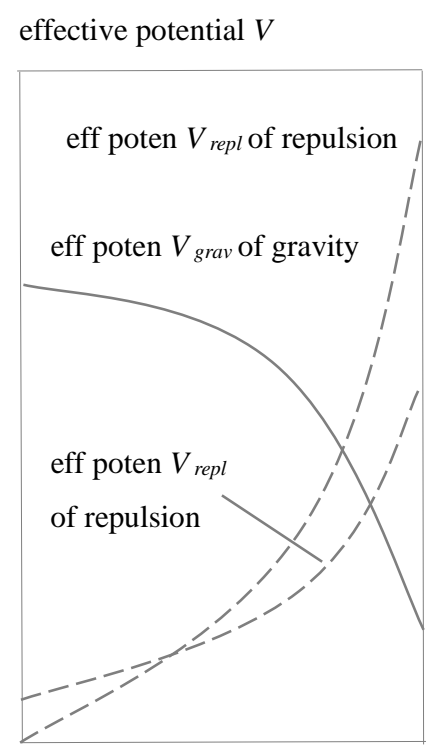

energy density $E$

Fig 5 Eff Poten and Engergy Density effective potential $V$

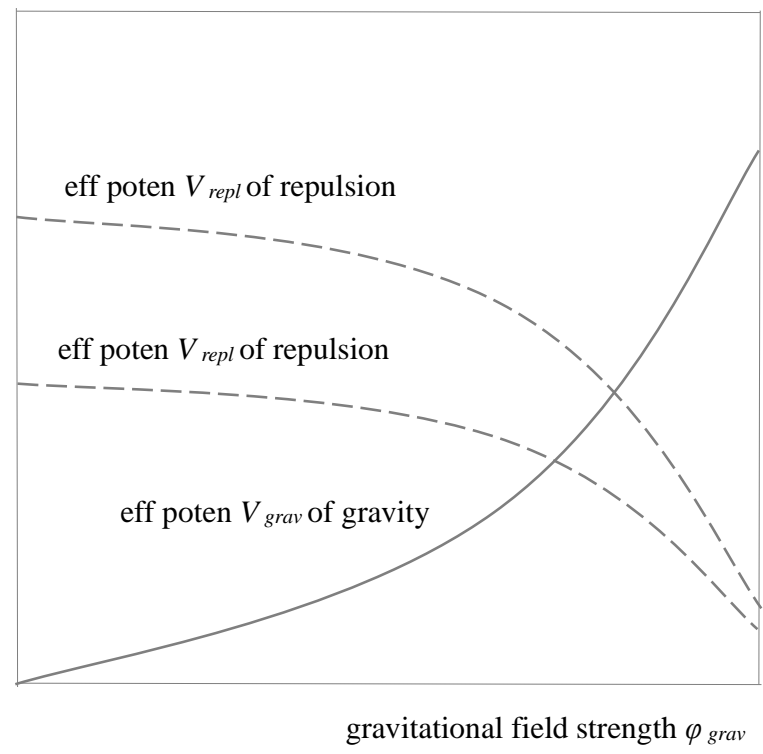

Fig 6 Eff Poten and Gravitational Field Strength

$$
E+i(-h v)=h v+i(-E), \quad p+i\left(-\frac{h}{\lambda} p_{0}\right)=\frac{h}{\lambda} p_{0}+i(-p)
$$

At every point in arche space-time, there is a high dimensional hypergraviton (extreme high negative energy with positive pressure) intertwined with a high dimensional hyperrepulson (extreme high positive energy with negative pressure), forming the arche-conjugate pulsaton.

At every point in the space-time of the first phase transition of the universe, there is a quantum graviton (very high negative energy with positive pressure) interwined with a quantum repulson (very high positive energy with negative pressure), forming a quantum conjugate pulsaton.

At every point of the space-time in the middle and early stage of the cosmic inflation, there is a hadron (negative energy with positive pressure) intertwined with a repulson of dark energy (positive energy with negative pressure), forming a strong action pulsaton.

\section{Super-Hedge Force of Arche-Conjugation}

Now, by literature [110] - [113], we can introduce double complex numbers and double complex functions, so as to expand the double complex symmetric gravity in theory and put forth the super unification (arche-conjugate) of quantum gravity and quantum repulsion.

\section{(9. 1 ) Double Complex Number}

In addition to the common complex or elliptic complex $a+i b$, there are also the double complex or hyperbolic complex $a+\varepsilon b$. here, $\varepsilon$ is the imaginary unit, $\varepsilon^{2}=+1$. Different from the common complex field $C$, all hyperbolic complex $a+\varepsilon b$ (which are real numbers) only form a commutative ring $H$, not a field. 
Definition 1 let $J$ denote pure double virtual units, which can be $J=i\left(i^{2}=-1\right)$ or can be $J=\varepsilon\left(\varepsilon^{2}=+1\right)$. If $a_{n}$ is a real number and the series $\sum_{n=0}^{\infty}\left|a_{n}\right|$ converges, then $a(J)=\sum_{n=0}^{\infty} a_{n} J^{2 n}$ is called a double real number. $a(J)$ corresponds to a pair of dual real numbers $\left(a_{C}, a_{H}\right)$, where

$$
a_{C}=a(J=i)=\sum_{n=0}^{\infty}(-1)^{n} a_{n}, \quad a_{H}=a(J=\varepsilon)=\sum_{n=0}^{\infty} a_{n}
$$

Definition 2 if both $a(J)$ and $b(J)$ are double real numbers, then $Z(J)=a(J)+J b(J)$ can be called double complex numbers. $Z(J)$ corresponds to a dual complex pair $\left(Z_{C}, Z_{H}\right)$, where

$$
Z_{C}=Z(J=i)=a_{C}+i b_{C}, \quad Z_{H}=Z(J=\varepsilon)=a_{H}+\varepsilon b_{H}
$$

Let $D C$ represent the set of all the double complex, that is,

When $D C=Z(J=i), D C$ represents the common complex field; when $D C=Z(J=\varepsilon), D C$ represents the hyperbolic complex ring.

For any two double complex $Z_{1}, Z_{2} \in D C$, the following algorithms are specified:

$$
\begin{aligned}
& Z_{1} \pm Z_{2}=\left(x_{1}+J y_{1}\right) \pm\left(x_{2}+J y_{2}\right)=\left(x_{1} \pm x_{2}\right)+J\left(y_{1} \pm y_{2}\right), \\
& Z_{1} \cdot Z_{2}=\left(x_{1}+J y_{1}\right) \cdot\left(x_{2}+J y_{2}\right)=\left(x_{1} x_{2}+J^{2} y_{1} y_{2}\right)+J\left(x_{1} y_{2}+y_{1} x_{2}\right),
\end{aligned}
$$

here, $\operatorname{Re} Z(J)=x(J)$ and $\operatorname{Im} Z(J)=y(J)$ can be called the real part and imaginary part of double complex number $Z(J)$ respectively.

Therefore, there are the following formulas:

$$
\begin{aligned}
& \operatorname{Re}\left(Z_{1} \pm Z_{2}\right)=\operatorname{Re} Z_{1} \pm \operatorname{Re} Z_{2} \\
& \operatorname{Im}\left(Z_{1} \pm Z_{2}\right)=\operatorname{Im} Z_{1} \pm \operatorname{Im} Z_{2} \\
& \operatorname{Re}\left(Z_{1} \cdot Z_{2}\right)=\operatorname{Re} Z_{1} \cdot \operatorname{Re} Z_{2}+J^{2} \operatorname{Im} Z_{1} \cdot \operatorname{Im} Z_{2} \\
& \operatorname{Im}\left(Z_{1} \cdot Z_{2}\right)=\operatorname{Re} Z_{1} \cdot \operatorname{Im} Z_{2}+J^{2} \operatorname{Im} Z_{1} \cdot \operatorname{Re} Z_{2}
\end{aligned}
$$

For the division operation of double complex $Z(J)$, it is closely related to the operative law of the conjugate number $\bar{Z}(J)$ of $Z(J)$.

Let a double complex number be $Z(J)=x(J)+J \cdot y(J)$, then

$$
\bar{Z}(J)=x(J)-J \cdot y(J)
$$

is called the conjugate number of $Z(J)$, while 


$$
|Z(J)|=\sqrt{|Z \bar{Z}|}=\sqrt{\left|x^{2}(J)-J^{2} y^{2}(J)\right|},
$$

is called the mold of $Z(J)$, and the double complex with zero mold is call the zero modulus double complex.

( 9.2 ) Double Complex Function

Without losing generality, the following only gives the functions of two real independent variables $(x, y)$, which can be extended to functions with $n$ real independent variables.

Definition 3 If the function

$$
E=E(J)=E(x, y ; J)=F(x, y ; J)+J \Omega(x, y ; J)
$$

on $N \subset R^{2}$ satisfies the following conditions, it is called a double complex function.

(a) $E_{C}=F_{C}+i \Omega_{C}$ and $E_{H}=F_{H}+\Omega_{H}$ are common complex functions and hyperbolic complex functions respectively, where

$$
\begin{array}{cc}
F_{C}=F_{C(x, y)}=F_{(J=i)}, & F_{H}=F_{H(x, y)}=F_{(J=\varepsilon)}, \\
\Omega_{C}=\Omega_{C(x, y)}=\Omega_{(J=i)}, & \Omega_{H}=\Omega_{H(x, y)}=\Omega_{(J=\varepsilon)},
\end{array}
$$

all are real functions that define on $N$.

(b) there is an analytic relation between $E_{C}$ and $E_{H}$, that is, if $x$ and $y$ are constants and $J$ are formal real independent variables, then $F(J)$ and $\Omega(J)$ are real analytic functions of $J$, which can be expressed as

$$
F(J)=\sum_{n=0}^{\infty} a_{n} J^{2 n}, \quad \Omega(J)=\sum_{n=0}^{\infty} b_{n} J^{2 n},
$$

where $a_{n(x, y)}$ and $b_{n(x, y)}$ are real functions, and the series $\Sigma|a|$ and $\Sigma|b|$ are convergent in the region.

\section{( 9.3 ) Super Unification of Arche-Conjugation}

As an extension of the double complex symmetric gravity, we now introduce the super unification of arche-conjugate in theory

Let $M(J)=\left(M_{C}, M_{H}\right)$ denote a double complex Riemann manifold, and the double complex symmetry gauge on it is expressed as

$$
g_{\mu \nu}(J)=A_{\mu \nu}(J)+J B_{\mu \nu}(J),
$$

where $A_{\mu \nu}(J)$ and $B_{\mu \nu}(J)$ are double real symmetric tensors. Thus, the real differential homeomorphism symmetry of the standard Riemann geometry is extended to the complex differential homeomorphism symmetry under the transformation of double complex coordinate $Z^{\mu}(J)=x^{\mu}(J)+J y^{\mu}(J)$.

If it is used to represent double complex frame:

$$
e_{\mu}^{a}(J)=\operatorname{Re}\left(e_{\mu}^{a}(J)\right)+J \operatorname{Im}\left(e_{\mu}^{a}(J)\right),
$$


then $g_{\mu v}(J)$ can be expressed as

$$
g_{\mu \nu}(J)=e_{\mu}^{a}(J) e_{v}^{b}(J) \eta_{a b},
$$

the corresponding double complex symmetric connection is

$$
\Gamma_{\mu \nu}^{\lambda}(J)=\Delta_{\mu \nu}^{\lambda}(J)+J \Omega_{\mu \nu}^{\lambda}(J),
$$

where $\Delta_{\mu \nu}^{\lambda}(J)$ and $\Omega_{\mu \nu}^{\lambda}(J)$ are the double real symmetric tensor. The double complex symmetric connection can be determined by the following equation:

$$
g_{\mu v ; \lambda}=\partial_{\lambda} g_{\mu \nu}-g_{\rho \nu} g_{\mu \lambda}^{\rho}-g_{\mu \rho} \Gamma_{\nu \lambda}^{\rho}=0,
$$

In this way, the double curvature tensor can be expressed as

$$
R_{\mu v \sigma}^{\lambda}(J)=-\partial_{\sigma} \Gamma_{\mu \nu}^{\lambda}(J)+\partial_{\nu} \Gamma_{\mu \sigma}^{\lambda}(J)+\Gamma_{\rho \nu}^{\lambda}(J) \Gamma_{\mu \sigma}^{\rho}(J)-\Gamma_{\rho \sigma}^{\lambda}(J) \Gamma_{\mu \nu}^{\rho}(J),
$$

Furthermore, the following double Ricci curvature tensors can be obtained by index contraction:

$$
R_{\mu \nu}(J):=R_{\mu v \sigma}^{\sigma}(J)=Q_{\mu \nu}(J)+J P_{\mu \nu}(J),
$$

and we can get four double complex Bianchi identities:

$$
\left(R^{\mu v}(J)-\frac{1}{2} g^{\mu v}(J) R\right)_{v}=0,
$$

Select the following double real actions:

$$
\begin{aligned}
S_{g}(J)= & \frac{1}{2} \int d^{4} x(J)\left[\Theta^{\mu v}(J) R_{\mu v}(J)+\left(\Theta^{\mu v}(J) R_{\mu v}(J)\right) \dagger\right] \\
& =\int d^{4} x(J)\left[\Xi^{\mu v}(J) Q_{\mu v}(J)+J^{2} \Pi^{\mu v}(J) P_{\mu v}(J)\right],
\end{aligned}
$$

where $\Theta^{\mu v}(J):=\sqrt{-g(J)} g^{\mu v}(J)=\Xi^{\mu v}(J)+J \Pi^{\mu v}(J)$, the symbol $\dagger$ denotes complex conjugation. Through the variation of $\Xi^{\mu v}(J)$ and $\Pi^{\mu v}(J)$ by $S_{g}(J)$, the vacuum field equation can be obtained:

$$
Q_{\mu \nu}(J)=0, \quad P_{\mu \nu}(J)=0,
$$

this is equivalent to the complex field equation: $R_{\mu v}(J)=0$.

\section{Repulsion and Gravitation behind Cosmic Inflation}

For the big bang, inflation and subsequent expansion of the universe, some studies have associated dark energy. For the researchers of dark energy, they often think of the cosmological constant in Einstein's field equation ${ }^{[14]-[116]}$. For cosmological constants, there has always been a so-called fine tuning problem.

It is generally accepted that the vacuum energy is equivalent to the cosmological constant in 
physical effect ${ }^{[114]-[116]}$. In astronomical observation, if vacuum energy exists, it should always be less than the critical density of the universe, that is:

$$
\rho_{\text {vac }}^{o b s} \leq \rho_{c} \approx 10^{-47} \mathrm{GeV}^{4}
$$

Through some estimates, the ground state energy (quantum zero point energy) density of a free scalar field with mass $m$ is preliminarily obtained:

$$
\rho_{v a c}^{t h}=\frac{1}{(2 \pi)^{3}} \int_{0}^{k_{c}} \frac{1}{2} \sqrt{k^{2}+m^{2}} 4 \pi k^{2} d k \approx \frac{1}{16 \pi^{2}} k_{c}^{4}
$$

here, $k_{c}$ represents the upper limit of the frequency. If the relevant theory is applicable to Planck energy scale $m_{p} \sim 10{ }^{19} \mathrm{GeV}$, then the density value of vacuum energy can be estimated as: $\rho_{\text {vac }}^{\text {th }} \sim 10^{74} \mathrm{GeV}^{4}$. It is not difficult to find that the observed value of vacuum energy is 121 orders of magnitude smaller than the theoretical value.

For cosmological constants, there are also so-called cosmic coincidence problems. In order to effectively alleviate the fine regulation brought by the coincidence problem in the universe, some researchers have considered the scalar field $\phi$ and the specific potential energy $V(\phi)$ in the energy -moment tensor. Here, the Lagrangian of a scalar field $\phi$ is

$$
S=\int d^{4} x \sqrt{-g}\left[\frac{\varepsilon}{2} \partial_{\mu} \phi \partial^{\mu} \phi-V(\phi)\right]
$$

where, $V(\phi)$ is the potential of scalar field, when $\varepsilon=1$, it means the quantum scalar field; when $\varepsilon=-1$, it means the phantom scalar field. For the variation of action ( 165 ), the energy-moment tensor of scalar field can be obtained:

$$
T^{\mu v}(\phi)=\varepsilon \partial^{\mu} \phi \partial^{v} \phi-g^{\mu \nu}\left[\frac{\varepsilon}{2} \partial_{\mu} \phi \partial^{\mu} \phi-V(\phi)\right]
$$

Both the huge magnitude gap problem in the fine regulation of vacuum energy and the potential energy problem in the fine regulation of cosmic coincidence, they all suggest to us on the other hand that at the beginning of the creation of the universe, the huge magnitude gap contains huge repulsive energy. The quantum repulsion must be closely related to the high-dimensional superrepulsion, and the quantum gravity must be closely related to the high-dimensional supergravity. The quantum conjugate relation is formed spontaneously between the quantum repulsion and the quantum gravity, and the arche-conjugate relation is formed spontaneously between the high-dimensional super repulsion and the high-dimensional super gravity. We can reduce the pseudo vacuum energy considered in inflationary universe model, Higgs field predicted by the standard physical model and dark energy inferred by the observation cosmology to the positive energy system with strong negative pressure, and they can also be reduced to the repulsive force (all factors resistant to the gravity) which forms the conjugate relationship with the gravity.

For the inflationary universe, the repulsion can be theoretically realized by the kinetic energy term of the inflationary field, which is the known k-inflation skyrocketing model ${ }^{[42]-[43]}$. In the period of the inflation, the energy of the universe is very high, and the effect of gravity is relatively large. By general 
relativity, gravity is described by the geometry of space-time. Now, we research the k-inflation model in noncommutative space-time based on the uncertainty principle of space-time. The results show that, in this model ${ }^{[48]-[49]}$, all disturbance modes are generated in the horizon, and the non commutative effect of space-time contributes to the power spectrum index of disturbance in the form of linear term. Compared with the experimental observation, it is found that the model can better meet the latest data.

Relevant research shows that ${ }^{[117][118]}$, the reason why large-scale structure was formed in the middle and late stage of the universe is that during the period of inflation, the quantum perturbations of the inflation field (also known as the inflation oscillator) and the spatiotemporal background field (known as the gauge field) were rapidly stretched out of the horizon and stopped evolving, and then evolved into classic perturbations and were preserved, and finally became the seed of the formation of large-scale structure. In fact, the inflation may never end, which is called the eternal inflation ${ }^{[18]-[120]}$. In many experiments, detecting the B-mode of photon polarization has been taken as the primary task. It is shown that only tensor perturbation can contribute to B-mode, while scalar perturbation only contributes to E-mode. Detection of B-mode is equivalent to detection of tensor disturbance, or detection of primary gravitational wave. The well-known BICEP2 project is based on their detection of disturbed B-mode. The data shows that ${ }^{[36][121]}$, the tensor-scale ratio is limited to $r=0.20_{-0.05}^{+0.07}$ and the possibility of $r=0$ is excluded at the confidence level of 7.0 $\sigma$. Based on these data, we can limit and exclude all kinds of the inflation models, including simple chaos inflation to complex multi-field models.

For the inflation of the commutative universe, some studies consider that a complex kinetic energy term is called irregular kinetic energy, and such a model is called K-inflation ${ }^{[117][118]}$. For example, Tachyon model ${ }^{[122]-[125]}$, Dirac-born-Infeld model ${ }^{[46][47]}$, all belong to this category.

At the time of the inflation, the energy scale of the universe is very high, which can be compared with the grand unification or even the Planck energy scale. Therefore, when it comes to the calculation of the inflation field, some appropriate corrections from quantum gravity are needed. As one of the most promising candidates of quantum gravity, superstring theory should give the necessary correction methods. In fact, nonperturbative superstring (or $\mathrm{m}$ ) theory points out that any physical process, when the interaction distance is very small, should meet the uncertainty relationship: $\Delta t_{p} \Delta x_{p} \geq l_{s}^{2}$.

Now, we consider introducing the interaction of repulsion and gravitation into the inflation model in noncommutative space-time.

First, consider the repulsion field behind the inflation. if the kinetic energy term of a single scalar field $\varphi$ is expressed as $X=-\frac{1}{2} g^{\mu \nu} \nabla_{\mu} \varphi \nabla_{\nu} \varphi$, then the most general repulsive action used to describe the field can be expressed as:

$$
S_{\varphi}=\int d^{4} X \sqrt{-g}\left[\frac{R}{2}+p_{\text {repl }}(\varphi, X)\right],
$$

here $p_{\text {repl }}(\varphi, X)$ is an arbitrary function of the scalar field $\varphi$ itself and its kinetic energy, and the definition of $X$ and the above repulsive action ensure that the motion equation of the field is second order. The energy momentum tensor of the inflation field can be obtained by the variation of the 
repulsion action.

$$
\begin{gathered}
K_{\mu \nu} \equiv-\frac{2}{\sqrt{-g}} \frac{\delta S_{\varphi}}{\delta g^{\mu \nu}}=p_{\text {repl }, X} \nabla_{\mu} \varphi \nabla_{\nu} \varphi+p_{\text {repl }} g_{\mu \nu}, \\
p_{\text {repl }, X} \equiv \frac{\partial p_{r e p l}(\varphi, X)}{\partial X},
\end{gathered}
$$

It is also equal to an ideal fluid, as long as its energy-momentum tensor is expressed as

$$
K_{\mu v}=\left(\sigma+p_{\text {repl }}\right) U_{\varphi, \mu} U_{\varphi, v}+p_{\text {repl }} g_{\mu v} .
$$

The $p_{\text {repl }}$ appearing here is the pressure $\left(p_{\text {repl }}<0\right)$ defined of repulsion in the co-moving coordinate system, while the energy density defined in the same coordinate system is:

$$
\sigma=2 X p_{r e p l, X}-p_{r e p l} .
$$

In addition, the definition of four velocity $U_{\varphi, \mu}$ is $U_{\varphi, \mu}=-\nabla_{\mu} \varphi / \sqrt{2 X}$.

Second, consider the gravitational field behind the inflation. if the kinetic energy term of a tensor field $\theta$ is expressed as $x=-\frac{1}{2} g^{\mu \nu} \nabla_{\mu} \theta \nabla_{\nu} \theta$, then the most general gravitation action used to describe the field can be expressed as:

$$
S_{\theta}=\int d^{4} x \frac{1}{\sqrt{-g}}\left[\frac{2}{R}+p_{\text {grav }}(\theta, x)\right],
$$

here $p_{\text {grav }}(\theta, x)$ is an arbitrary function of the tensor field $\varphi$ itself and its kinetic energy, and the definition of $x$ and the above gravitational action ensure that the motion equation of the field is second order. The energy momentum tensor of the inflation field can be obtained by the variation of the gravitational action.

$$
\begin{gathered}
T_{\mu \nu} \equiv-\frac{1}{2} \sqrt{-g} \frac{\delta S_{\theta}}{\delta g^{\mu \nu}}=p_{\text {grav }, X} \nabla_{\mu} \theta \nabla_{\nu} \theta+p_{\text {grav }} g_{\mu \nu}, \\
p_{\text {grav }, X} \equiv \frac{\partial p_{\text {grav }}(\theta, x)}{\partial x},
\end{gathered}
$$

It is also equal to an ideal fluid, as long as its energy-momentum tensor is expressed as

$$
T_{\mu \nu}=\left(\rho+p_{\text {grav }}\right) U_{\theta, \mu} U_{\theta, v}+p_{\text {grav }} g_{\mu \nu} .
$$

The $p_{\text {grav }}$ appearing here is the pressure ( $p_{\text {grav }}>0$ ) of gravity defined in the co-moving coordinate system, while the energy density defined in the same coordinate system is:

$$
\rho=2 x p_{\text {grav }, x}-p_{\text {grav }} \text {. }
$$


In addition, the definition of four velocity $U_{\theta, \mu}$ is $U_{\theta, \mu}=-\nabla_{\mu} \theta / \sqrt{2 x}$.

Now, consider the interaction between the repulsive field and the gravitational field behind the inflation. In this case, the most general hedging action between the repulsive field and the gravitational field behind the inflation field can be expressed as follows:

$$
S_{\varphi}-S_{\theta}=\int d^{4} X \sqrt{-g}\left[\frac{R}{2}+p_{\text {repl }}(\varphi, X)\right]-\int d^{4} x \frac{1}{\sqrt{-g}}\left[\frac{2}{R}+p_{\text {grav }}(\theta, x)\right]=\pi_{s},
$$

where, $\pi_{s} \gg 0$.

By the variation of the action, the energy momentum tensor of the hedging action behind the inflation field can be obtained

$$
\begin{aligned}
K_{\mu \nu}-T_{\mu \nu} \equiv & -\frac{2}{\sqrt{-g}} \frac{\delta S_{\varphi}}{\delta g^{\mu \nu}}+\frac{1}{2} \sqrt{-g} \frac{\delta S_{\theta}}{\delta g^{\mu \nu}} \\
= & p_{\text {repl }, X} \nabla_{\mu} \varphi \nabla_{\nu} \varphi+p_{\text {repl }} g_{\mu \nu}-p_{\text {grav }, X} \nabla_{\mu} \theta \nabla_{\nu} \theta-p_{\text {grav }} g_{\mu v} \\
& p_{\text {repl }, X}-p_{\text {grav }, x} \equiv \frac{\partial p_{\text {repl }}(\varphi, X)}{\partial X}-\frac{\partial p_{\text {grav }}(\theta, x)}{\partial x}
\end{aligned}
$$

It is equivalent to a fluid, as long as its hedging energy momentum tensor is expressed as

$$
K_{\mu \nu}-T_{\mu \nu}=\left(\sigma+p_{\text {repl }}\right) U_{\varphi, \mu} U_{\varphi, v}+p_{\text {repl }} g_{\mu \nu}-\left(\rho+p_{\text {grav }}\right) U_{\theta, \mu} U_{\theta, v}-p_{\text {grav }} g_{\mu \nu} .
$$

For an uniform and homogeneous flat universe ( space curvature $K=0$ ) can be described by Friedmann-Robertson-Walker (FRW) gauge in the form of $d s^{2}=-d t^{2}+a^{2}(t) d x^{2}$. In this way, the kinetic energy

$$
X-x=\frac{1}{2}\left(\dot{\varphi}^{2}-\dot{\theta}^{2}\right),
$$

of the inflation field and the noncommutative relation of time and space become: $\Delta t \Delta x \geq l_{s}^{2} / a(t)$.

In order to make the non commutative relation meaningful at any time, we must introduce a new time coordinate $\tau: d \tau=a(t) d t$, the gauge also changes. Now, there is a good definition of non commutative relation of time and space: $\Delta \tau \Delta x \geq l_{s}^{2}$.

By introducing FRW gauge into the repulsive field equation in the inflation, non Friedmann equation related to repulsive field can be obtained:

$$
H_{r e p l}^{2}=\frac{\sigma}{3}=\frac{1}{3}\left(\dot{\varphi}^{2} p_{r e p l, x}-p_{r e p l}\right)
$$

and by introducing FRW gauge into the gravitational field equation in the inflation, Friedmann equation 
related to gravitational field can be obtained:

$$
H_{\text {grav }}^{2}=\frac{\rho}{3}=\frac{1}{3}\left(\dot{\theta}^{2} p_{\text {grav }, X}-p_{\text {grav }}\right),
$$

Thus we can set up the hedge-expansive universe equation reflecting the interaction between repulsion and gravity:

$$
H_{\text {repl }}^{2}-H_{\text {grav }}^{2}=\frac{1}{3}(\sigma-\rho)=\frac{1}{3}\left(\dot{\varphi}^{2} p_{\text {repl }, x}-p_{\text {repl }}-\dot{\theta}^{2} p_{\text {grav }, X}+p_{\text {grav }}\right) .
$$

By the variation of the repulsion action ( 167 ), the equation of motion related to repulsion in the inflation field can be obtained

$$
\ddot{\varphi}\left(p_{r e p l, X}+\dot{\varphi}^{2} p_{r e p l, X X}\right)+3 H_{r e p l} \dot{\varphi} p_{r e p l, X}=p_{r e p l, \varphi}-\dot{\varphi}^{2} p_{r e p l, X \varphi} .
$$

In addition, there are the equation

$$
\dot{H}_{r e p l}=-X p_{r e p l, X}=-\frac{1}{2} \dot{\varphi}^{2} p_{r e p l, X}
$$

Because of the irregular kinetic energy term in function $p_{\text {repl }}$, the dispersion relation of the inflation field is modified, and the perturbation mode of quantum repulsion no longer moves at the speed of light. The propagation velocity of repulsive disturbance can be characterized by the velocity of sound defined by the following formula:

$$
c_{s, r e p l}^{2} \equiv \frac{p_{r e p l, X}}{\sigma_{X}}=\frac{p_{r e p l, X}}{p_{r e p l, X}+\dot{\varphi}^{2} p_{r e p l, X X}} \leq 1 .
$$

Back to the regular case: $p_{\text {repl }}(\varphi, X)=X-V(\varphi), V(\varphi)$ is the repulsive potential energy of the inflation field.

By the variation of the gravitational action ( 172 ), the equation of motion related to gravitation in the inflation field can be obtained

$$
\ddot{\theta}\left(p_{\text {grav }, x}+\dot{\theta}^{2} p_{\text {grav }, x x}\right)+3 H_{\text {grav }} \dot{\theta} p_{\text {grav }, x}=p_{\text {grav }, \varphi}-\dot{\theta}^{2} p_{\text {grav }, x \varphi} .
$$

In addition, there are the equation

$$
\dot{H}_{\text {grav }}=-x p_{\text {grav }, x}=-\frac{1}{2} \dot{\theta}^{2} p_{g r a v, x}
$$

Because of the irregular kinetic energy term in function $p_{\text {grav }}$, the dispersion relation of the inflation field is modified, and the perturbation mode of quantum gravitation no longer moves at the speed of light. The propagation velocity of gravitational disturbance can be characterized by the velocity of sound defined by the following formula:

$$
c_{s, \text { grav }}^{2} \equiv \frac{p_{\text {grav }, x}}{\rho_{x}}=\frac{p_{\text {grav }, x}}{p_{\text {grav }, x}+\dot{\theta}^{2} p_{\text {grav }, x x}} \leq 1 .
$$

Back to the regular case: $p_{\text {grav }}(\theta, x)=x-V(\theta), V(\theta)$ is the gravitational potential energy of the inflation field. 
By combining equation ( 185 ) with equation ( 187 ), the motion equation of the hedging between repulsion and gravitation in the inflation field can be obtained

$$
\begin{gathered}
\ddot{\varphi}\left(p_{\text {repl }, X}+\dot{\varphi}^{2} p_{\text {repl }, X X}\right)-\ddot{\theta}\left(p_{\text {grav }, x}+\dot{\theta}^{2} p_{g r a v, x x}\right)+3\left(H_{r e p l} \dot{\varphi} p_{\text {repl }, X}-H_{\text {grav }} \dot{\theta} p_{g r a v, x}\right) \\
=p_{\text {repl }, \varphi}-\dot{\varphi}^{2} p_{r e p l, X \varphi}-p_{\text {grav }, \varphi}+\dot{\theta}^{2} p_{\text {grav }, X \varphi} .
\end{gathered}
$$

In addition, there is

$$
\dot{H}_{r e p l}-\dot{H}_{g r a v}=-X p_{r e p l, X}-x p_{g r a v, x}=-\frac{1}{2} \dot{\varphi}^{2} p_{r e p l, X}+\frac{1}{2} \dot{\theta}^{2} p_{g r a v, x}
$$

The propagation velocity of hedging disturbance can be characterized by the velocity of sound defined by the following formula:

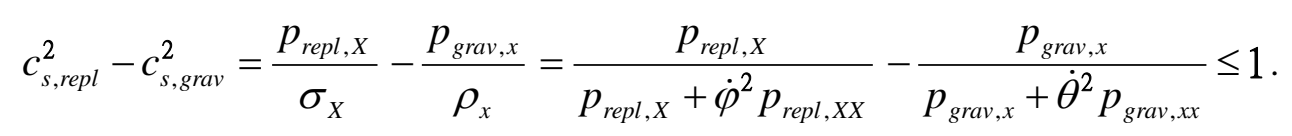

Back to the regular case:

$$
p_{\text {repl }}(\varphi, X)-p_{\text {grav }}(\theta, x)=X-x-V(\varphi)+V(\theta)
$$

$V(\varphi)-V(\theta)$ is the hedge-potential energy of the inflation field, the speed of sound is equal to $c_{s, \text { repl }}^{2}-c_{s, \text { grav }}^{2}=1$.

Therefore, we can define several slow-rolling parameters to describe the process of the inflation:

$$
\begin{gathered}
\varepsilon_{1}=-\frac{\dot{H}_{\text {repl }}}{H_{\text {repl }}^{2}}+\frac{\dot{H}_{\text {grav }}}{H_{\text {grav }}^{2}}=\frac{X p_{\text {repl }, X}}{H_{\text {repl }}^{2}}-\frac{x p_{\text {grav }, x}}{H_{\text {grav }}^{2}}, \\
\varepsilon_{2}=\frac{\dot{\varepsilon}_{1}}{\varepsilon_{1}\left(H_{\text {repl }}-H_{\text {grav }}\right)}, \\
s=\frac{\dot{c}_{s, r e p l}-\dot{c}_{s, \text { grav }}}{c_{s, \text { repl }} H_{\text {repl }}-c_{s, \text { grav }} H_{\text {grav }}},
\end{gathered}
$$

They are small in the period of the inflation, i.e. $\left|\varepsilon_{1}\right|,\left|\varepsilon_{2}\right|, s \leq 1$.

It can be inferred that in the early evolution of the universe, the interaction between quantum repulsion and quantum gravity led to a big rip and a big rebound. Then it is concluded that in the initial stage of the big rip, quantum repulsion and quantum gravity restrain each other, leading to the slowing down of the big bang of the universe; and at the end of the big rip, quantum repulsion and quantum gravity form a superposed big rebound, leading to the super inflation of the universe.

\section{Perturbation of Repulsive Scalar and Gravitational Tensor}

On the one hand, the scalar disturbance is considered as the showing of repulsive effect, on the other hand, the tensor disturbance is considered as the showing of gravitational effect. In the initial stage of the big rip, due to the mutual restraint of quantum repulsion and quantum gravity, the scalar 
and tensor perturbations are not obvious. However, at the end of the big rip, the scalar and tensor perturbations are very significant, and there is a large power spectral exponential run.

Let $V$ be the volume of space, and the repulsive action of scalar disturbance can be given by

$$
S_{\varphi}=\frac{V}{2} \int_{k<k_{0}} d \eta d^{3} k \approx z^{2}(\eta)\left(\zeta_{-k}^{\prime} \zeta_{k}^{\prime}-c_{s, r e p l}^{2} k^{2} \zeta_{-k} \zeta_{k}\right),
$$

where the apostrophe represents the differential of the new time coordinate $\eta$, which is defined as:

$$
\frac{d \eta}{d \tau} \equiv a_{e f f}^{-2}=\left(\frac{\beta_{k}^{-}}{k_{+}^{\beta}}\right)^{1 / 2}=\frac{1}{a^{2}}(\tau-\Delta \tau)
$$

here the function of $\beta_{k}^{ \pm}(\tau)$ is defined :

$$
\beta_{k}^{ \pm}(\tau)=a^{ \pm 2}(\tau-\Delta \tau), \quad \Delta \tau=l_{s}^{2} k
$$

where $\zeta_{k}$ is the curvature disturbance and $z \equiv a \sqrt{2 \varepsilon_{1}} / c_{s}$ is Mukhanov variable. The $\beta_{k}^{ \pm}(\tau)$ function used here is equivalent to the formula used in other literature in the sense of integral, and once the exact solution of the perturbed equation is obtained, the form of the $\beta_{k}^{ \pm}(\tau)$ function will not affect the final physical results. However, if the present form is chosen, the effect of non commutation of space-time can be highlighted to the greatest extent under the approximate conditions, and the perturbed equation will become easier to deal with. The equation of motion satisfied by the variable $\zeta_{k}$ is:

$$
u_{k}^{\prime \prime}+\left(c_{s}^{2} k^{2}-\frac{z^{\prime \prime}}{z}\right) u_{k}=0
$$

This equation can be obtained by the variation of action ( 195 ), where the modulus function is defined as $u_{k}=z \zeta_{k}$. By using the slow-rolling approximation condition and the slow-rolling parameters, the third coefficient of the disturbance equation ( 198 ) can be obtained:

$$
\frac{z^{\prime \prime}}{z} \approx \frac{1}{\eta^{2}}\left(1-\varepsilon_{1}\right)^{-2} \Sigma^{-2}(\tau, \Delta \tau)\left[2 \Sigma(\tau, \Delta \tau)\left(1+\frac{\varepsilon_{2}}{2}-s\right)-\varepsilon_{1}+\frac{\varepsilon_{2}}{2}-s\right] .
$$

The following relationships are used in the derivation:

$$
\eta \approx-\left[a(\tau-\Delta \tau) H(\tau-\Delta \tau)\left(1-\varepsilon_{1}\right)\right]^{-1},
$$

This relationship can be obtained by equation ( 196 ). In ( 199 ) function $\Sigma$ is defined as:

$$
\Sigma(\tau, \Delta \tau)=\frac{a(\tau)}{a(\tau-\Delta \tau)} \frac{H_{r e p l}(\tau-\Delta \tau)}{H_{r e p l}(\tau)} .
$$

It can be seen from the uncertainty principle of time and space that the time when all modes are generated is when the non-commutative relation of time and space takes the equal sign. At this moment, the co-moving wave number has the maximum value: 


$$
k_{0}(\tau)=\frac{a_{e f f}}{l_{s}}=\frac{a\left(\tau+\Delta_{\tau}\right)}{l_{s}} \approx \frac{H_{r e p l}(\tau)}{l_{s}}\left[a(\tau) H_{r e p l}^{-1}(\tau)-\Delta \tau\right]
$$

In other words, at time $\tau$, a mode of wave number to be $k_{0}$ is generated. Moreover, the value of $\lambda$ at this time can be estimated:

$$
\left.\lambda_{0} \equiv \frac{\Delta \tau}{a(\tau) H_{r e p l}^{-1}(\tau)-\Delta \tau}\right|_{k=k_{0}} \approx l_{s} H_{*}
$$

where $H_{*}^{-1}$ is called the Hubble horizon. In the period of the inflation, the scale factor $a(t)$ either evolves in the form of approximate exponential function, i.e. $a \sim e^{H t}$, or in the form of power-law function, i.e. $a \sim t^{n}$, where $n$ is a relatively large constant. At the same time, $H *$ hardly evolves. Thus, by equation ( 203 ), it can be seen that the parameter $\lambda$ decreases with time.

At time $\tau$, if a mode just crosses the horizon, it should have momentum

$$
k_{c}=a(\tau) \frac{H_{r e p l}(\tau)}{c_{s, r e p l}}
$$

then we can get:

$$
\frac{k_{c}}{k_{0}} \approx \frac{a(\tau)}{a(\tau)-H_{r e p l}(\tau) \Delta \tau} \frac{l_{s} H_{r e p l}(\tau)}{c_{s, r e p l}} \approx \frac{l_{s} H_{*}}{c_{s, r e p l}}
$$

In the following, the research will focus on the case of $l_{s} H * / c_{s, \text { repl }} \ll<1$, that is, all the modes are generated within the horizon $\left(k_{0}>>k_{c}\right)$. The reason for not considering another case $l_{s} H * / c_{s, \text { repl }} \geq 1$ is that it is difficult to explain the flatness of microwave background radiation. See literature [120] [118] for detailed discussion. Therefore, in the case of $l_{s} H_{*} / c_{s, \text { repl }} \ll<1$, it is very reasonable to treat $\lambda$ as a small quantity and retain its first-order term in the calculation. In this way, the power spectrum of the disturbance can be calculated at the time when the mode is out of the horizon ( $\left.k=a H_{\text {repl }} / c_{s, \text { repl }}\right)$.

In the case of slow-roll approximation, equation ( 198 ) is simplified to:

$$
u_{k}^{\prime \prime}+\left(c_{s, r e p l}^{2} k^{2}-\frac{v^{2}-1 / 4}{\eta^{2}}\right) u_{k}=0
$$

Take the initial condition as the Bunch-Davies vacuum defined as follows: $u_{k}=\frac{1}{\sqrt{2 c_{s, \text { repl }} k}} e^{-i c_{s, r e p l} k \eta}$.

The final solution of equation (206) is as follows:

$$
u_{k}(\eta)=\frac{\sqrt{\pi}}{2} e^{i(v+1 / 2) \pi / 2} \sqrt{-\eta} H_{v, \text { repl }}^{(1)}\left(-c_{s, r e p l} k \eta\right) .
$$

where, $H_{v, \text { repl }}^{(1)}$ represents the first kind of Hankel function related to repulsion. On the scale beyond the horizon, the solution is approximately: 


$$
u_{k}(\eta)=2^{v-3 / 2} e^{i(v-1 / 2) \pi / 2} \frac{\Gamma(v)}{\Gamma(3 / 2)} \frac{1}{\sqrt{2 c_{s, r e p l} k}}\left(-c_{s, r e p l} k \eta\right)^{1 / 2-v},
$$

Then, the original scalar perturbation spectrum is obtained:

$$
\begin{aligned}
P_{s} & =\frac{k^{3}}{2 \pi^{2}}\left|\zeta_{k}\right|^{2}=\frac{k^{3}}{2 \pi^{2}}\left|\frac{u_{k}}{z}\right|^{2} \\
& =\left.\frac{2^{2 v-4}}{\varepsilon_{1} c_{s, r e p l}}\left[\frac{\Gamma(v)}{\Gamma(3 / 2)}\right]^{2}\left(\frac{H}{2 \pi}\right)^{2}\left(\frac{c_{s, r e p l} k}{a H_{r e p l}}\right)^{3-2 v}\right|_{c_{s, r e p l} k=a H_{r e p l}} \approx \frac{1}{8 \pi^{2} \varepsilon_{1} c_{s, r e p l}} \frac{H_{r e p l}^{2}}{M_{p l}^{2}} c_{s, r e p l}{ }_{c=a H_{r e p l}}
\end{aligned}
$$

By the definition of the scalar disturbance spectrum index, we can get:

$$
n_{s}-1 \equiv \frac{d \ln P_{s}}{d \ln k}=3-2 v-s \approx-2 \varepsilon_{1}-\varepsilon_{2}+s+\frac{4}{3} \lambda,
$$

In addition, the spectral index runs as follows:

$$
\alpha_{s} \equiv \frac{d n_{s}}{d \ln k}=-2 \varepsilon_{2} \varepsilon_{1}-\varepsilon_{3} \varepsilon_{2}+s_{1} s-\frac{4}{3} \lambda(1+\lambda)\left(1+\varepsilon_{1}\right) \approx-\frac{4}{3} \lambda
$$

Some small quantities $\varepsilon_{3} \equiv \dot{\varepsilon}_{2} /\left(\varepsilon_{2} H_{\text {repl }}\right)$ and $s_{1} \equiv \dot{s} /\left(s H_{\text {repl }}\right)$ are defined here.

The motion equation of tensor perturbation related to repulsion is almost the same as that of scalar perturbation, but the definition of Mukhanov variable becomes $z=a$. The specific disturbance equation is as follows:

$$
v_{k}^{\prime \prime}+\left(k^{2}-\frac{a^{\prime \prime}}{a}\right) v_{k}=0
$$

where the modular function is defined as $v_{k} \equiv a h k / 2$. It should be noted that $h_{k}$ here represents two physical freedom degrees of tensor perturbation, namely $h_{+}$and $h_{x}$. Using the same approximation as scalar perturbation, we get:

$$
\frac{a^{\prime \prime}}{a} \approx \frac{1}{\eta^{2}}\left(1-\varepsilon_{1}\right)^{-2} \Sigma^{-2}(\tau, \Delta \tau)\left[2 \Sigma(\tau, \Delta \tau)-\varepsilon_{1}\right] \approx \frac{v^{2}-1 / 4}{\eta^{2}},
$$

The solution of equation ( 212 ) is obtained by keeping the value of $v$ to the linear term:

$$
v_{k}(\eta)=\frac{\sqrt{\pi}}{2} e^{i(v+1 / 2) \pi / 2} \sqrt{-\eta} H_{v, \text { repl }}^{(1)}(-k \eta) .
$$

The solution also satisfies the Bunch-Davies vacuum condition. On the scale beyond the horizon, the solution is approximately:

$$
u_{k}(\eta)=2^{v-3 / 2} e^{i(v-1 / 2) \pi / 2} \frac{\Gamma(v)}{\Gamma(3 / 2)} \frac{1}{\sqrt{2 k}}(-k \eta)^{1 / 2-v},
$$

then, the original tensor perturbation spectrum related to repulsion is obtained. 


$$
\begin{aligned}
P_{t} & =2 \times \frac{k^{3}}{2 \pi^{2}}\left|h_{k}\right|^{2}=\frac{k^{3}}{\pi^{2}}\left|\frac{2 v_{k}}{z}\right|^{2} \\
& =\left.\left.2^{2 v}\left[\frac{\Gamma(v)}{\Gamma(3 / 2)}\right]^{2}\left(\frac{H_{\text {grav }}}{2 \pi}\right)^{2}\left(\frac{k}{a H_{\text {grav }}}\right)^{3-2 v}\right|_{k=a H_{\text {grav }}} \approx \frac{2}{\pi^{2}} \frac{H_{\text {grav }}^{2}}{M_{p l}^{2}}\right|_{k=a H_{\text {grav }}} .
\end{aligned}
$$

By the definition of tensor disturbance spectrum index and tensor-scale ratio, we can get:

$$
n_{t} \equiv \frac{d \ln P_{t}}{d \ln k}=3-2 v \approx-2 \varepsilon_{1}+\frac{4}{3} \lambda, \quad r \equiv \frac{P_{t}}{P_{s}}=16 c_{s, r e p l} \varepsilon_{1}
$$

It is precisely because the $\lambda$ term appears in the above equation that a new kind of inflationary universe model with kinetic energy term based on the non commutative relation of space-time will be more consistent with the experimental observation than the related model in the commutative space-time. Next, by references [121] - [124], we can compare and analyze these models with the latest experimental observations.

In order to constrain the parameters (fast sub model and DBI model) in the K-inflation model in non commutative space-time, the latest observation data are used, including: microwave background radiation data in Planck experiment, supplemented by large-scale polarization data in WMAP ( hereinafter referred to as Planck + WP ); the large polar moment data of microwave background radiation ( hereinafter referred to as high L ); Planck gravitational lens power spectrum. Large scale structure ( hereinafter referred to as BAO ) data, etc. Specific data in reference [2]: scalar spectral index 0. $9583 \pm 0.0081($ Planck + WP ), $0.9633 \pm 0.0072$ ( Planck + WP + lensing ), $0.9570 \pm 0.0075$ $\left(\right.$ Planck + WP + high L ), $0.9607 \pm 0.0063($ Planck $+\mathrm{WP}+\mathrm{BAO})$, its running value $n{ }^{\prime}=-0.021 \pm$ $0.012($ Planck $+\mathrm{WP}), n{ }_{s}{ }_{s}=-0.017 \pm 0.012($ Planck $+\mathrm{WP}+$ lensing $), n_{s}^{\prime}=-0.022_{-0.010}^{+0.011} \quad($ Planck $+\mathrm{WP}+$ high $\mathrm{L}), n_{s}^{\prime}=-0.021_{-0.010}^{+0.012}($ Planck $+\mathrm{WP}+\mathrm{BAO}) ;$ and in reference [121]: tensor-scale ratio $r=0.20_{-0.05}^{+0.07}$. In addition, there are values given in reference [36]: $r=0.23_{-0.09}^{+0.05}$ and $n_{t}=0.03_{-0.11}^{+0.13}$.

For the inflation of tachyon, considering the case that $\mathrm{M}$ is large enough, and taking the e-folding number as a parameter, the following limitations are obtained by fitting:

$$
\lambda=0.0138_{-0.00409}^{+0.00404}, \quad N=34.64_{-4.149}^{+5.489} \quad(68 \% \mathrm{CL}) .
$$

For the DBI inflation model, the parameters are limited to:

$$
\lambda=0.0998_{-0.00368}^{+0.00363}, \quad N=59.61_{-7.287}^{+9.692} \quad(68 \% \mathrm{CL}) .
$$

Figure 7 provided in reference [121] shows the contour of parameters in the confidence interval of $1 \sigma$ and $2 \sigma$. It can be seen from Figure 7 that the e-folding number in the tachyon model is relatively small. If the value of e-folding number is required to be between 50 and 60 , the model can only be said to be in good accordance with the observation; however, in the DBI model, the value of e-folding number is enough to solve the flatness problem in the big bang cosmology.

It can be seen from the above discussion that repulsion and gravitation always interact in the big 
bang, inflation and subsequent expansion of the universe.
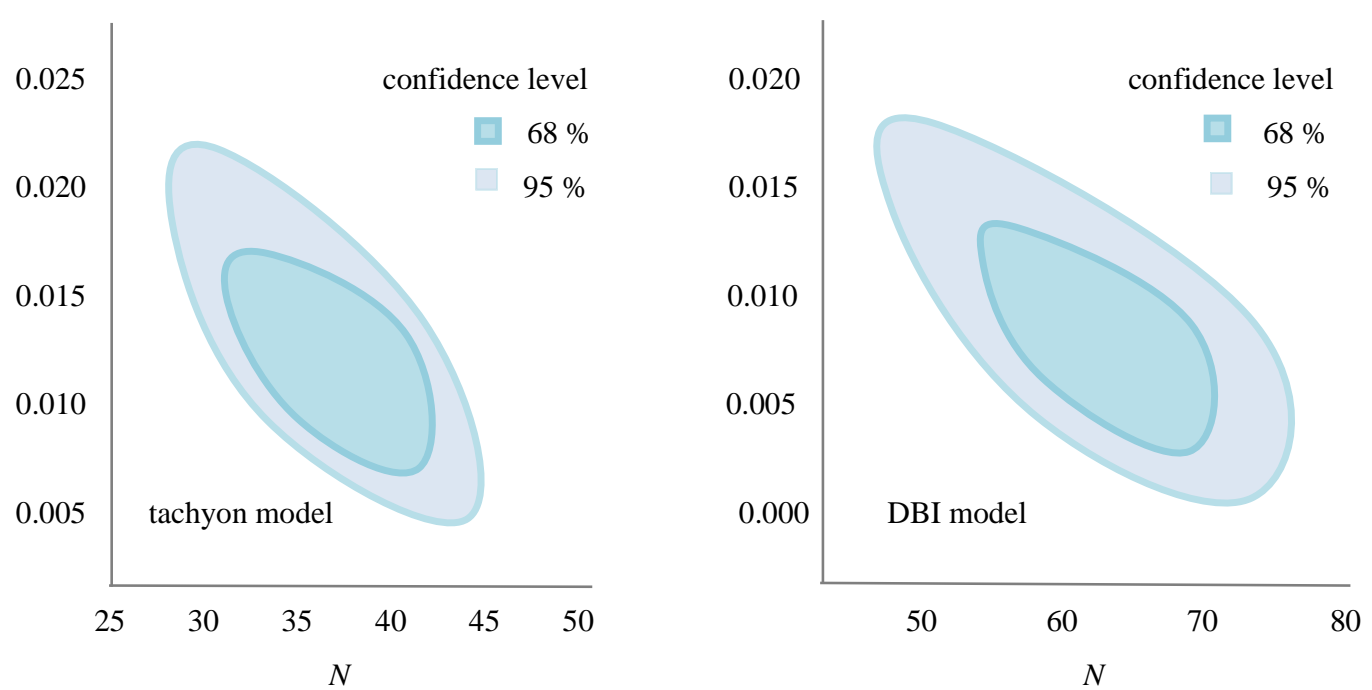

The center red dot represents the best fit value of the parameter.

Here, the left figure corresponds to the tachyon model; the right figure corresponds to the DBI model.

Fig. 7 contour of parameters $\lambda$ and $N$ in confidence intervals $1 \sigma$ and $2 \sigma$

\section{Path Integral of Hedge-Unified Quantum Field}

Now, we expand the research framework of quantum gravity theory and loop quantum gravity cosmology, and establish a path integral method of quantum hedge between quantum gravity and quantum repulsion. Therefore, we must fully consider the ultra-synergy between the whole big bang and the internal quantum gravity of the universe. It can not be neglected that this ultra-synergy is a powerful impetus for the creation of the quantum universe, while the subsequent ultra-synergy between the whole Big Bang and the internal gravity, the strong force, the electro-weak force of the universe, is a powerful impetus of the cosmic inflation.

In theory, we should explore the establishment of quantum cosmic-pulse dynamics, and then explore to establish the ultra-synergy physics of the quantum universe.

For the ultra-synergistic field of the quantum universe near Planck energy scale, it is necessary for us to consider improving and expanding the path integral of quantum gravity, establishing the path integral of ultra-synergy of quantum universe and its corresponding new historical summation scheme, so as to link the whole Big Bang with the internal quantum gravity of the universe.

We know that in quantum mechanics and quantum field theory, all physical laws can be expressed in the form of path integrals. For a single particle system, particles can arrive at events $\left(x_{2}, t_{2}\right)$ via any path from events $\left(x_{1}, t_{1}\right)$, where the weight of each path is $\exp (i I)$, I is the action of the system. So the probability of a particle arriving at a point $\left(x_{2}, t_{2}\right)$ from a point $\left(x_{1}, t_{1}\right)$ is

$$
<x_{2}, t_{2} \mid x_{1}, t_{1}>=\int \delta x \exp (i I)
$$

here the functional integral is performed on all paths connecting $\left(x_{1}, t_{1}\right)$ and $\left(x_{2}, t_{2}\right)$. 
This expression can also be used in quantum field theory. If we regard field $\phi(x)$ as the coordinate of field configuration space, then events can be given by point ( $\phi(x), t)$, which means that the at time $t$ the field has configuration $\phi(x)$, so that the probability amplitude of the field from $\left(\phi_{1}(x), t_{1}\right)$ to $\left(\phi_{2}(x), t_{2}\right)$ is

$$
<\phi_{2}(x), t_{2} \mid \phi_{1}(x), t_{1}>=\int \delta \phi(x, t) \exp (i I)
$$

The functional integral in the formula proceeds along all paths connecting $\left(\phi_{1}(x), t_{1}\right)$ and $\left(\phi_{2}(x)\right.$, $\left.t_{2}\right)$ in the configuration space. Thus, as long as the substitution $(x, t) \leftrightarrow(\phi(x), t)$ is made, the discussion of single particle system and the discussion of field are exactly the same in form.

At the starting point of quantum theory, the path integral formulation determines the state of the system by giving the wave function of the system in the appropriate configuration space. The construction of wave function should be based on its probability interpretation and can be written in

$$
\Psi(x, t)=N \int_{c} \delta x(t) \exp \{i I[x(t)]\}
$$

here $N$ is the normalization factor.

This formula can be directly extended to the case of quantum field. The ground state wave function of the system has a form:

$$
\Psi(\phi(x), \tau)=N \int \delta \phi(x) \exp \{-I[\phi(x)]\}
$$

In the original framework, the path integral representation has been used in quantum gravity by researchers. For generalized relativity, the gravitational field is the metric tensor field.

Under the framework of ultra-synergistic analysis of quantum universe proposed in this series, we can improve and expand the path integral expression of quantum gravity, and establish the path integral expression of quantum hedge-unified force for both quantum gravity and quantum repulsion.

Here, we introduce a spatiotemporal metric of compact four-dimensional manifold which reflects quantum gravity, and it can be written as

$$
d s_{\text {grav }}^{2}=-\left(N_{\text {grav }}^{2}-N_{\text {grav }, i} N_{\text {grav }}^{i}\right) d t^{2}+2 N_{\text {grav }, i} d x^{i} d t+h_{\text {grav }, i j} d x^{i} d x^{j}
$$

where the foot mark grav represents the quantity belonging to gravitation, $N_{\text {grav }}$ is a time-lapse function reflecting the quantum gravitation, $N_{\text {grav }, i}$ is a shift function reflecting quantum gravitation, $h_{\text {grav }, i j}$ is an intrinsic metric on three-dimensional space-like hypersurfaces under quantum gravitation, and $N_{\text {grav }}$, $N_{\text {grav }, i}, h_{\text {grav }, \text { ij }}$ are all functions of space-time coordinates.

For the universe, the coordinates of quantum gravitational conformation space should be $h_{\text {grav }, i j}$ and matter field $\theta$. The quantum universe is described by $\left(h_{\text {grav }, i j}, \theta\right)$. Thus, the transition probability amplitude of the universe from a three-dimensional space-like hypersurface $h_{g r a v i j}$ ( there is quantum gravitational field $\theta$ on it ) to a space-like hypersurface $h_{g r a v, i j}^{\prime}\left(\right.$ there is field $\theta^{\prime}$ on it ) can be expressed as

$$
<h_{g r a v, i j}^{\prime}, \theta^{\prime} \mid h_{g r a v, i j}, \theta>=\int \delta\left[g_{\mu \nu}, \theta\right] \exp \left(i I\left[g_{\mu \nu}, \theta\right]\right)
$$

here $I\left[g_{\mu \nu}, \theta\right]$ is the action of Euclidean space related to gravitation.

Similar to the treatment of general quantum systems, the wave function of quantum gravity can be 
expressed as

$$
\Psi\left[h_{\text {grav }, i j}, \theta\right]=N \int_{c} \delta g_{\mu \nu} \delta \theta \exp \left\{i I\left[g_{\mu \nu}, \theta\right]\right\}
$$

here, $N$ is the normalized constant, and the integral region $C$ is all the paths of the connective points $\left(h_{\text {grav }, i j}, \theta\right)$ and the initial points in the gravitational configuration space. The ground-state wave function of quantum gravity has the following form:

$$
\Psi\left[h_{\text {grav }, i j}, \theta\right]=N \int_{c} \delta g_{\mu \nu} \delta \theta \exp \left\{-I\left[g_{\mu \nu}, \theta\right]\right\}
$$

It can be expected that the wave function ( 226 ) under quantum gravity should satisfy a cosmic dynamical equation similar to Schrodinger equation. The following equation belongs to this kind of equation, which can be regarded as Wheeler-de Witt equation in the original framework of loop quantum gravity cosmology.

Under the one-cycle (also called semi-classical WKB) approximation, formula ( 226 ) becomes

$$
\Psi\left[h_{\text {grav }, i j}, \theta\right]=N \sum_{i} B_{i} \exp \left(-I_{\text {grav }, c l}^{i}\right)
$$

here, $I_{\text {grav }, c l}$ is the $i$-th classical Euclidean gravitational action which satisfies the principle of minimum action. $N$ is the normalized constant, and $B_{i}$ is the fluctuation of classical orbit.

By extending the original framework of quantum gravity, the path integral expression can be further applied to quantum repulsion. In the case beyond general relativity, the repulsive field is the field corresponding to the gravitational field.

Now, in the quantum noncommutative space-time of the universe, we can establish the path integral expression of quantum repulsion.

Here, we introduce a four-dimensional manifold space-time metric that reflects quantum repulsion

$$
d s_{r e p l}^{2}=-\left(N_{r e p l}^{2}-N_{r e p l, i} N_{r e p l}^{i}\right) d t^{2}+2 N_{r e p l, i} d x^{i} d t+h_{r e p l, i j} d x^{i} d x^{j}
$$

where the foot mark repl represents the quantity belonging to repulsion, $N_{\text {repl }}$ is a time-lapse function reflecting the quantum repulsion, $N_{\text {repl, } i}$ is a shift function reflecting quantum repulsion, $h_{\text {repl, } i j}$ is an intrinsic metric on three-dimensional space-like hypersurfaces under quantum repulsion, and $N_{\text {repl }}$, $N_{\text {repl }, i}, h_{\text {repl }, \text { ij }}$ are all functions of space-time coordinates.

For the universe, the coordinates of quantum repulsive conformation space should be $h_{\text {repl, }, i j}$ and matter field $\varphi$. The quantum universe is described by $\left(h_{r e p l i j}, \varphi\right)$. Thus, the transition probability amplitude of the universe from a three-dimensional space-like hypersurface $h_{\text {repl,ij }}$ ( there is quantum repulsive field $\varphi$ on it ) to a space-like hypersurface $h_{r e p l, i j}^{\prime}$ ( there is field $\varphi^{\prime}$ on it ) can be expressed as

$$
<h_{r e p l, i j}^{\prime}, \varphi^{\prime} \mid h_{r e p l, i j}, \varphi>=\int \delta\left[g_{\mu \nu}, \varphi\right] \exp \left(i I\left[g_{\mu \nu}, \varphi\right]\right)
$$

here $I\left[g_{\mu \nu}, \varphi\right]$ is the action of Euclidean space related to repulsion.

Similar to the treatment of general quantum systems, the wave function of quantum repulsion can be expressed as

$$
\Psi\left[h_{r e p l, i j}, \varphi\right]=N \int_{c} \delta g_{\mu \nu} \delta \varphi \exp \left\{i I\left[g_{\mu \nu}, \varphi\right]\right\}
$$

here, $N$ is the normalized constant, and the integral region $C$ is all the paths of the connective points 
$\left(h_{r e p l, i j}, \varphi\right)$ and the initial points in the repulsive configuration space. The ground-state wave function of quantum repulsion has the following form:

$$
\Psi\left[h_{r e p l, i j}, \varphi\right]=N \int_{c} \delta g_{\mu \nu} \delta \varphi \exp \left\{-I\left[g_{\mu \nu}, \varphi\right]\right\}
$$

It can be expected that the wave function ( 230 ) under quantum repulsion should satisfy a cosmic dynamical equation similar to Schrodinger equation. The following equation belongs to this kind of equation, which can be seen as Wheeler-de Witt equation in the current framework of loop quantum gravity cosmology.

Under the one-cycle (also called semi-classical WKB) approximation, formula ( 230 ) becomes

$$
\Psi\left[h_{r e p l, i j}, \varphi\right]=N \sum_{i} D_{i} \exp \left(-I_{\text {repl }, c l}^{i}\right)
$$

here, $I^{i}{ }^{i}$ epl, $c l$ is the $i$-th classical Euclidean repulsive action which satisfies the principle of minimum action. $N$ is the normalized constant, and $D_{i}$ is the fluctuation of classical orbit.

Next, the original framework of quantum gravity is further expanded. The path integral expression can be further applied to both quantum gravity and quantum repulsion, and then the quantum gravity and quantum repulsion are combined to give the path integral expression of quantum hedging. In the case beyond general relativity and loop quantum gravity theory, the interaction between quantum repulsive field and quantum gravitational field forms quantum hedge-unified field.

Now, in the quantum noncommutative space-time of the universe, we can establish the path integral expression of quantum hedge-unified field. For the inflation of the early universe, we should consider not only the local inwards-pull of quantum gravity, but also the whole outwards-push of quantum repulsion.

Here, we introduce a four-dimensional manifold space-time metric that reflects quantum hedging:

$$
\begin{aligned}
d s_{\text {repl }}^{2}-d s_{\text {grav }}^{2}= & -\left(N_{\text {repl }}^{2}-N_{\text {repl }, i} N_{\text {repl }}^{i}\right) d t^{2}+2 N_{\text {repl }, i} d x^{i} d t+h_{\text {repl }, i j} d x^{i} d x^{j} \\
& +\left(N_{\text {grav }}^{2}-N_{\text {grav }, i} N_{\text {grav }}^{i}\right) d t^{2}-2 N_{\text {grav }, i} d x^{i} d t-h_{\text {grav }, i j} d x^{i} d x^{j}
\end{aligned}
$$

where the foot mark repl represents the quantity belonging to repulsion, $N_{\text {repl }}$ is a time-lapse function reflecting the quantum repulsion, $N_{\text {repl, } i}$ is a shift function reflecting quantum repulsion, $h_{\text {repl, } i j}$ is an intrinsic metric on three-dimensional space-like hypersurfaces under quantum repulsion, and $N_{\text {repl }}$, $N_{r e p l, i}, h_{\text {repl, } i j}$ are all functions of space-time coordinates, the foot mark grav represents the quantity belonging to gravitation, $N_{\text {grav }}$ is a time-lapse function reflecting the quantum gravitation, $N_{\text {grav }, i}$ is a shift function reflecting quantum gravitation, $h_{\text {grav, ij }}$ is an intrinsic metric on three-dimensional space-like hypersurfaces under quantum gravitation, and $N_{\text {grav }}, N_{\text {grav }, i}, h_{\text {grav }, i j}$ are all functions of space-time coordinates.

For the universe, the coordinates of quantum hedging-conformation space should be $h_{\text {repl,ij }}$ and $h_{\text {grav }, i j}$, repulsive matter field $\varphi$ and gravitational matter field $\theta$. The quantum universe is described by $\left(h_{\text {repl, }, i j}-h_{\text {grav }, i j}, \varphi-\theta\right)$. Thus, the transition probability amplitude of the universe from a threedimensional space-like hypersurface $h_{\text {repl }, i j}-h_{\text {grav }, i j}$ ( there is quantum hedge-unified field $\Xi=\varphi-\theta$ on it ) to a space-like hypersurface $h^{\prime}{ }_{r e p l, i j}-h^{\prime}{ }_{\text {grav }, i j}$ ( there is field $\varphi^{\prime}-\theta^{\prime}$ on it ) can be expressed as

$$
<h_{i j}^{\prime}, \Xi\left|h_{i j}, \Xi>=<h_{r e p l, i j}^{\prime}-h_{g r a v, i j}^{\prime}, \varphi^{\prime}-\theta^{\prime}\right| h_{r e p l, i j}-h_{g r a v, i j}, \varphi-\theta>
$$




$$
=\int \delta\left[g_{\mu \nu}, \varphi\right] \exp \left(i I\left[g_{\mu \nu}, \varphi\right]\right)-\int \delta\left[g_{\mu \nu}, \theta\right] \exp \left(i I\left[g_{\mu \nu}, \theta\right]\right)
$$

here $I\left[g_{\mu \nu}, \Xi\right]$ is the action of Euclidean space related to quantum hedging.

Similar to the treatment of general quantum systems, the wave function of quantum hedging can be expressed as

$$
\begin{aligned}
\Psi\left[h_{i j}, \Xi\right] & =\Psi\left[h_{r e p l, i j}-h_{\text {grav }, i j}, \varphi-\theta\right] \\
& =N \int_{c} \delta g_{\mu \nu} \delta \varphi \exp \left\{i I\left[g_{\mu \nu}, \varphi\right]\right\}-N \int_{c} \delta g_{\mu \nu} \delta \theta \exp \left\{i I\left[g_{\mu \nu}, \theta\right]\right\}
\end{aligned}
$$

here, $N$ is the normalized constant, and the integral region $C$ is all the paths of the connective points $\left(h_{\text {replij }}-h_{\text {grav }, i j}, \varphi-\theta\right)$ and the initial points in the hedge-unified configuration space. The ground-state pulsating function of quantum hedging has the following form:

$$
\begin{aligned}
\Psi\left[h_{i j}, \Xi\right] & =\Psi\left[h_{r e p l, i j}-h_{\text {grav }, i j}, \varphi-\theta\right] \\
& =N \int_{c} \delta g_{\mu \nu} \delta \varphi \exp \left\{-I\left[g_{\mu \nu}, \varphi\right]\right\}-N \int_{c} \delta g_{\mu \nu} \delta \varphi \exp \left\{-I\left[g_{\mu \nu}, \varphi\right]\right\}
\end{aligned}
$$

It can be expected that the wave function ( 234 ) under quantum repulsion should satisfy a cosmic dynamical equation.

Under the one-cycle (also called semi-classical WKB) approximation, formula ( 234 ) becomes

$$
\begin{aligned}
\Psi\left[h_{i j}, \Xi\right]= & \Psi\left[h_{r e p l, i j}-h_{\text {grav }, i j}, \varphi-\theta\right] \\
& =N \sum_{i} D_{i} \exp \left(-I_{\text {repl }, c l}^{i}\right)-N \sum_{i} B_{i} \exp \left(-I_{\text {grav }, c l}^{i}\right)
\end{aligned}
$$

\section{Ultra-Synergy Dynamical Equation of Quantum Universe}

For the ultra-synergy between the whole Big Bang and the internal quantum gravity of the universe, and the subsequent ultra-synergy between the whole Big Bang and the internal gravity, strong force, electro-weak force of the universe, we first give the Hamiltonian form of general relativity, so as to establish the pulse dynamics equation of the quantum universe.

For this reason, we introduce scalar functional:

$$
I=I_{B}+I_{S}+I_{B S}=\int\left(L_{B}+L_{S}+L_{B S}\right) \sqrt{-g} d^{4} x
$$

where

$I_{B}$ and $L_{B}$ respectively represent the (pull outwards) action of the cosmic big bang and its Lagrange function.

$I_{S}$ and $L_{S}$ respectively represent the (draging inwards) action of gravity and its Lagrange function.

$I_{B S}$ and $L_{B S}$ respectively represent the interaction and its Lagrange functions between the cosmic big bang and gravity.

The expression of $I_{S}$ and $L_{S}$ is taken as 


$$
L_{S}=R, L_{B}=-2 k L_{B} .
$$

where $k$ is the Einstein gravitational constant, $k=8 \pi G / c^{4}$.

Thus the variational principles is given by $\delta I=\delta\left(I_{B}+I_{S}+I_{B S}\right)=0$, the variations of internal quantum gravitational action in the universe are obtained:

$$
\delta I_{S}=\delta \int \sqrt{-g} R d^{4} x=\int \sqrt{-g}\left(R_{\mu v}-\frac{1}{2} g_{\mu v} R\right) \delta g^{\mu v} d^{4} x
$$

The variation $\delta I_{B}$ of the global extrapolation action $I_{B}$ can be known from ( 235 ) and ( 236 ):

$$
\delta I_{B}=-2 \mathrm{k} \delta \int \sqrt{-g} L_{B} d^{4} x
$$

According to variational calculus, we can obtain

$$
\delta I_{B}=-2 k \int\left\{\frac{\partial}{\partial g^{\mu \nu}}\left(\sqrt{-g} L_{B}\right)-\left[\frac{\partial}{\partial g^{\mu \nu} \lambda}\left(\sqrt{-g} L_{B}\right)\right]_{\lambda}\right\} \delta g^{\mu v} d^{4} x
$$

Defining energy-momentum tensor $T_{B, \mu v}$ :

$$
T_{B, \mu \nu}=\frac{1}{\sqrt{-g}} 2\left\{\frac{\partial}{\partial g^{\mu \nu}}\left(\sqrt{-g} L_{B}\right)-\left[\frac{\partial}{\partial g_{\lambda}^{\mu \nu}}\left(\sqrt{-g} L_{B}\right)\right]_{\lambda}\right\}
$$

we can obtain

$$
\delta I_{B}=-k \int \sqrt{-g} T_{B, \mu v} \delta g^{\mu v} d^{4} x
$$

The variation $\delta I$ of the ultra-synergy $I$ can be known from ( 237 ) and ( 241 ):

$$
\delta I=\delta\left(I_{B}+I_{S}+I_{B S}\right)=\int \sqrt{-g}\left(R_{\mu \nu}-\frac{1}{2} g_{\mu \nu} R-k T_{B, \mu \nu}\right) \delta g^{\mu \nu} d^{4} x
$$

Let $\delta\left(I_{B}+I_{S}+I_{B S}\right)=0$, considering the arbitrariness of $\delta g^{\mu \nu}$, we obtain

$$
R_{\mu \nu}-\frac{1}{2} g_{\mu \nu} R=k T_{B, \mu \nu}
$$

If the interaction term is introduced into ultra-synergy quantity $I$

$$
I_{B S}=c \int \sqrt{-g} d^{4} x
$$

here $c$ is an undetermined constant, then $\delta I_{B S}=\frac{c}{2} \int g_{\mu \nu} \delta g^{\mu o} \sqrt{-g} d^{4} x$, by $\delta\left(I_{B}+I_{S}+I_{B S}\right)=0$, we obtain

$$
R_{\mu v}-\frac{1}{2} g_{\mu v} R+\lambda g_{\mu v}=k T_{B, \mu v}
$$

here $\lambda=\frac{c}{2}$, it can be regarded as a classical ultra-synergy field equation with cosmic terms.

Furthermore, in order to establish the quantum cosmic pulse-dynamical equation, the action quantity of gravitational field is taken as 


$$
I_{S}=\frac{1}{16 \pi}\left[\int_{M} d^{4} x \sqrt{-g}(R-2 \Lambda)+2 \int_{2 M} d^{3} x \sqrt{h} K\right]
$$

This ntegral is carried out on the surface $\partial M$ of spatiotemporal manifold $M$. Here, $h=\operatorname{det} h_{i j}, K$ $=h_{i j} K^{i j}, h_{i j}$ and $K_{i j}$ are intrinsic metric tensor and external curvature tensor on three-dimensional boundary respectively, $R$ is scalar curvature, $\Lambda$ is cosmic constant.

For the ultra-synergy, note the metric representation ( 235 ), which can be written as follows:

$$
I=I_{B}+I_{S}+I_{B S}=I_{B}+\frac{1}{16} \pi \int d^{4} x \sqrt{h} N\left(K_{i j} K^{i j}-K^{2}+{ }^{3} R-2 \Lambda\right)+I_{B S}
$$

here

$$
K_{i j}=\frac{1}{N}\left(-\frac{1}{2} \frac{\partial h_{i j}}{\partial t}+N_{(i \mid j)}\right)
$$

The subscript small vertical bar represents covariant derivatives for $h_{i j}$, and ${ }^{3} R$ is an internal curvature scalar given by $h_{i j}$.

The expression of Hamiltonian of ultra-synergistic system can be obtained from ( 247 ):

$$
H=\int d^{3} x\left(\pi \dot{N}+\pi^{i} \dot{N}_{i}+N H^{0}+N_{i} H^{i}\right)
$$

here $N$ and $N^{i}$ act as Lagrange multiplier:

$$
\begin{gathered}
H^{0}=\frac{1}{16 \pi} \sqrt{\pi}\left[K_{i j} K^{i j}-K^{2}-{ }^{3} R(h)+2 \Lambda\right], \quad H^{i}=-2 \pi^{i j}{ }_{\mid j} \\
\pi=\frac{\partial S_{g}}{\delta \dot{N}}=0, \quad \pi^{i}=\frac{\partial S_{g}}{\delta \dot{N}_{i}}=0, \quad \pi^{i j}=\frac{\partial S_{g}}{\delta \dot{h}_{i j}}=\frac{1}{16 \pi} \sqrt{h}\left(K h^{i j}-K^{i j}\right) .
\end{gathered}
$$

Because $\pi=0$ and $\pi^{i}=0$ are invariably valid, $\dot{\pi}=0, \dot{\pi}^{i}=0$. By the Hamiltonian equation, we obtain

$$
H^{0}=0, \quad H^{i}=0 .
$$

Formula ( 252 ) expresses Hamiltonian constraint and momentum constraint.

The following metric is introduced into the hyperspace consisting of the configuration $\left\{h_{i j}\right\}$ of ultra-synergy field :

$$
G_{i j k l}=\frac{1}{2} \sqrt{h}\left(h_{i k} h_{j l}+h_{i l} h_{j k}-h_{i j} h_{k l}\right)
$$

then ( 250 ) can be written as

$$
H^{0}=\frac{1}{16 \pi}\left[G_{i j k l} \pi^{i j} \pi^{k l}-\sqrt{h}\left({ }^{3} R-2 \Lambda\right)\right]
$$

An operator processing is given: $\pi^{i j} \rightarrow \frac{1}{i} \frac{\delta}{\delta h_{i j}}$. If there exists a Big Bang and ultra-synergy field, the related generalized momentum is replaced by an operator, then the Hamiltonian constraint is given: 


$$
\left\{-G_{i j k l} \frac{\delta^{2}}{\delta h_{i j} \delta h_{k l}}+\sqrt{h}\left[-{ }^{3} R+2 \Lambda+16 \pi T_{B S}\left(\frac{1}{i} \frac{\delta}{\delta \phi}, \phi\right)\right]\right\} \Psi\left[h_{i j}, \phi\right]=0
$$

This is the extended Wheele-De Witt equation, which is the quantum cosmic pulse-dynamical equation we want to establish. In this equation, $T_{B S}$ is the component of the energy momentum tensor of the interaction field in the normal direction of the three-dimensional space-like hypersurface. This pulsing function with ultra-synergy is not obviously time-dependent, so there is no time derivative term in the equation.

By the momentum constraints, we obtain

$$
\left(\frac{\delta}{\delta h_{i j}} \Psi\right)_{\mid j}=T^{B S, n i} \Psi\left(h_{i j}, \phi\right)
$$

This is the momentum constraint equation. It shows that for different metric $h_{i j}$ obtained by coordinate transformation under ultra-synergy, its pulsating function must be the same.

Equations ( 255 ) and ( 256 ) are variational equations in infinite dimensional space, here there is no general and strict method for solving them. Only by limiting the number of degrees of freedom in hyperspace, that is, by using a small hyperspace model (hyperspace model only with a finite degree of freedom), we can limit quantum fluctuations to preserve the degrees of freedom on some topologies and a few geometric features of space-time. The variational equation is simplified to a much simpler set of partial differential equations.

The quantum cosmic pulsating-function $\Psi\left(h_{i j}, \phi\right)$ should satisfy the equations ( 255$)$ and ( 256 ), $|\Psi|^{2}$ expresses the probability of quantum cosmos appearing at points $\left(h_{i j}, \phi\right)$ in hyperspace.

We can consider ( 255 ) as an extension of the Schrodinger equation of zero energy steady state.

For the ultra-synergistic field of vacuum or in cosmological principles, ( 256 ) formula becomes

$$
\left[\frac{\delta}{\delta h_{i j}} \Psi\left(h_{i j}, \phi\right)\right]_{\mid j}=0
$$

This formula shows that the small change of the coordinate system on the three-dimensional surface will cause the small change of the scale, which will lead to the change of the pulsating function with the ultra-synergy to zero, which means that the ultra-pulsating function is gauge-invariant.

Functional differential equation ( 255 ) can be regarded as a differential equation with $G_{i j k l}$ as hypermetric on manifold $\left\{h_{i j}\right\}$ of ultra-synergy metric field. All infinite three-dimensional geometries $\left\{h_{i j}\right\}$ and ultra-synergistic media together form an infinite dimensional configuration space, which can be called hyperspace.

By the research in [118], let $V$ represent the potential energy function of the inflation field, and assume that it has the following form:

$$
V(\phi)=\lambda \sum_{n=1}^{\infty} a_{n} \phi^{2 n}
$$

Here $\lambda$ is a proportional coefficient, which will be determined by the amplitude of the power spectrum. Define slow-rolling parameters: 


$$
\varepsilon=\frac{M_{p l}^{2}}{2}\left(\frac{V^{\prime}}{V}\right)^{2}, \quad \eta=M_{p l}^{2} \frac{V^{\prime \prime}}{V^{\prime}}, \quad \xi=M_{p l}^{4} \frac{V^{\prime} V^{\prime \prime}}{V^{2}}
$$

where the apostrophe is the differential of the field $\phi$, the power spectrum index $n_{s}$ and its running $n_{s}^{\prime}$ of the scalar disturbance can be expressed as:

$$
\begin{gathered}
n_{s}-1 \approx 2 \eta-6 \varepsilon, \\
n_{s}^{\prime} \approx 16 \varepsilon \eta-24 \varepsilon^{2}-2 \xi .
\end{gathered}
$$

It can be seen from the above analysis that $\xi$ should not be too small if there is a large spectral index running, at least in the order of $\varepsilon$ or $\eta$. Suppose the following relationship is true:

$$
\xi=\alpha \varepsilon+(\beta-\alpha) \eta
$$

where $\alpha$ and $\beta$ are two undetermined constants. Therefore, the coefficients in the potential energy ( 257 ) must meet certain conditions to meet the formula ( 261 ).

The power spectrum of scalar disturbance is given by the following formula:

$$
P_{s}=A_{s}\left(\frac{k}{k_{*}}\right)^{n_{s}-1+n_{s} \ln \left(k / k_{*}\right) / 2},
$$

where $A_{s}$ is its amplitude, the spectral index $n_{s}$ is given by the formula ( 259 ), and the perturbation $n_{s}^{\prime}$ $=d n_{s} / d \ln k$ of the spectral index is given by the formula ( 260 ). Using equation ( 261 ), equation ( 260 ) can also be written as:

$$
n_{s}^{\prime}=16 \varepsilon \eta-24 \varepsilon^{2}-2 \alpha \varepsilon-2(\beta-\alpha) \eta,
$$

The tensor-scale ratio $r$ is given by the relation $r \approx 16 \varepsilon$. It can be seen from formula ( 258 ) that the parameter $\alpha$ is very important for the running of spectral index, as shown in Figure 8, $n_{s}^{\prime} \sim O(\varepsilon) \sim O($ $10^{-2}$ ), and $r \geq 0.20$. That is to say, a large tensor-scale ratio and a large spectral index run are realized at the same time.

At present, the latest observation data include: in reference [121], scaling ratio $r=0.20_{-0.05}^{+0.07}$; microwave background radiation data in Planck experiment, supplemented by large-scale polarization data in WMAP (hereinafter referred to as Planck+WP); large polar moment data of microwave background radiation (hereinafter referred to as high L); power spectrum of Planck gravitational lens, data of large-scale structure, etc. Specific data in reference [2], scalar spectral index $0.9583 \pm 0.0081$ (Planck +WP), 0. $9633 \pm 0.0072$ (Planck +WP + lensing), 0.9570 \pm 0.0075 (Planck + WP + highL), 0. $9607 \pm 0.0063$ (Planck $+\mathrm{WP}+\mathrm{BAO})$, its running value $n_{s}^{\prime}=-0.021 \pm 0.012($ Planck $+\mathrm{WP}), n_{s}^{\prime}=-0$. $017 \pm 0.012$ (Planck + WP + lensing), $n_{s}^{\prime}=-0.022_{-0.010}^{+0.011}($ Planck $+\mathrm{WP}+$ highL $), n_{s}^{\prime}=-0.021_{-0.010}^{+0.012}$ (Planck $+\mathrm{WP}+\mathrm{BAO})$.

In addition, there are other research teams that give their results. For example, document [126] combines background imaging of chemical extragalactic polarisation (B2), Planck and WP data set to give $r=0.23_{-0.09}^{+0.05}$; document [127] combines data from Superova legacy Survey (SNLS) to give $r=0.20_{-0.05}^{+0.04}$; document [128] combines data from Planck, supernova union 2.1, Bao and bicep2 to 
give $r=0.199_{-0.044}^{+0.037}$, and document [129] combines data from other Bao to give $r=0.20_{-0.06}^{+0.04}$. It is also shown that the detected photon polarization B mode can not be completely explained by topological defects or large external dimension model [129] [130]. The most likely explanation for the large scale ratio is the tensor disturbance model produced in the period of inflation.

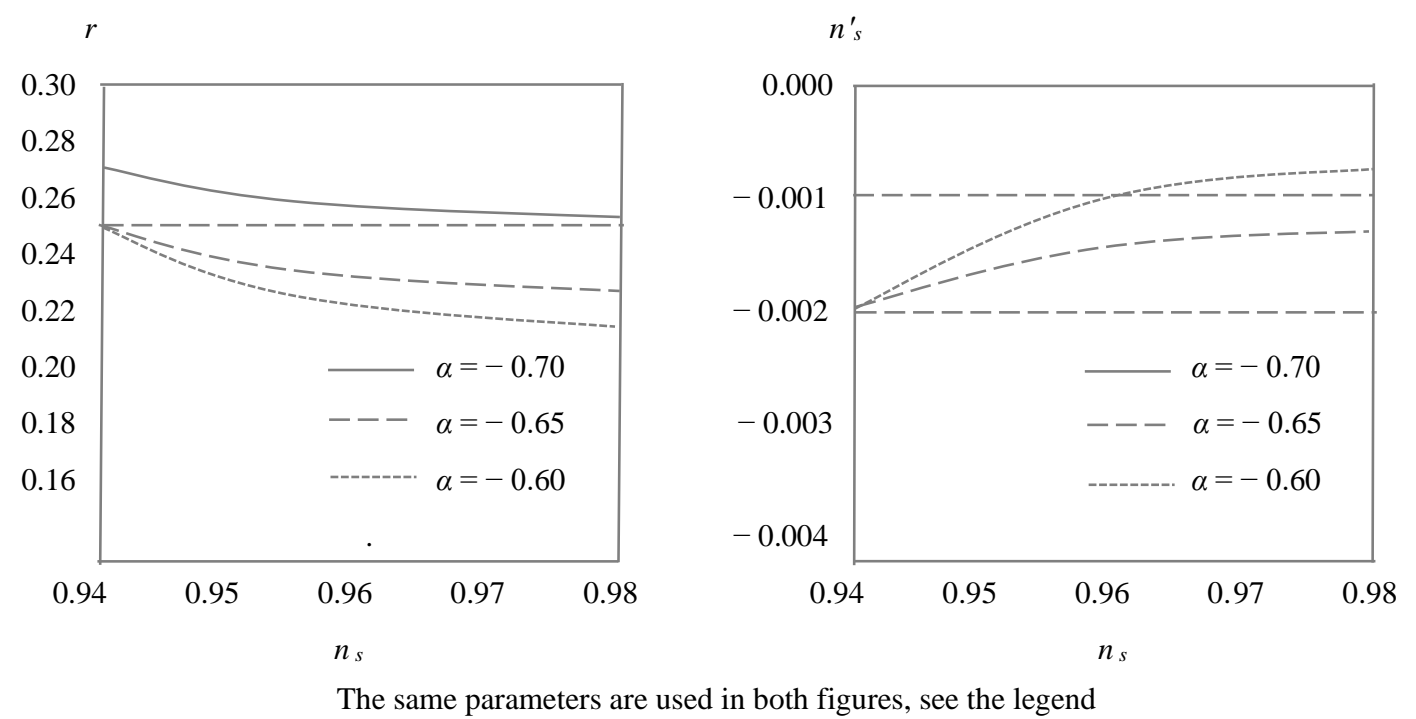

Fig. 8 the relationship between the scale ratio $r$ and the spectral index $n_{s}$ on the left; the relationship between the spectral index run $n_{s}$ and the spectral index $n_{s}$ on the right

Using these data and results, the parameters $\alpha$ and $\beta$ in the model are limited. The characteristic scale of inflation field in the period of inflation is $\phi / M_{p l} \approx O$ ( 10 ), so it should belong to the category of inflation. The power spectrum amplitude is given by Planck,

$$
A_{s}\left(k=0.002 \mathrm{Mpc}^{-1}\right)=2.215 \times 10^{-19}{ }^{[131]},
$$

the value of parameter $\lambda$ can be estimated:

$$
\lambda \approx \frac{3 \pi^{2}}{2}\left(\sum_{n=1}^{\infty} a_{n} \phi^{2 n}\right)^{-1} r A_{s} \approx 6.02 \times 10^{-11},
$$

where the best fitting values of parameters $\alpha$ and $\beta$ are used.

\section{Basic Equation of Cosmic Paradigm Dynamics}

The perturbation method and space-time background dependence of string theory involve a more profound problem: in the conception of string theory today, because string theory relies on background, it describes perturbation expansion of fixed space-time background, so it may not be the real basis $^{[51]-[53]}$. Some physicists regard background independence as a basic requirement for a theory of quantum gravity. It is especially true since general relativity has been background independent ${ }^{[132]-[133]}$.

In the ultra-synergistic paradigm established in this series, both quantum space-time and classical space-time are ultra-synergy dynamical systems. We will now discuss the establishment of a dynamic basis for quantum space-time evolution. 
In view of the cosmic ecosphere of the Multi-verse World (MUW), we make the following new considerations:

The compactness of space-time dimension is first the arche-event of the initial universe (the problem of cosmic physics), then the derivative event of the basic unit of matter (the problem of particle physics).

To break through the compactness of space-time dimension must have a very powerful force. The higher the space-time dimension is, the greater the force needed to break through the compactness is. The force created by the Big Bang of the universe is just enough to break through the compactness of four-dimensional space-time.

In view of the cosmic ecosphere of the Multi-verse World (MUW), we further make the following new considerations:

In the initial stage of the creation of the universe, the gravitation in four-dimensional space-time was once very strong, and its intensity was even close to quantum gravity. However, at present, the gravitation in four-dimensional space-time is far weaker than the other three interactions in fourdimensional space-time. This reason is the Big Bang of the universe and the subsequent inflation of the universe. This Big Bang and inflation constantly break through the compactness of four-dimensional space-time, and then weaken the role of gravity, making gravity weaker and weaker.

The description, analysis and explanation in this section are mainly applicable to the period from Planck time $\left(10^{-44} \mathrm{sec}\right)$ to the beginning of inflation $\left(10^{-36} \mathrm{sec}\right.$ or $\left.10^{16} \mathrm{GeV}\right)$ :

In the Big Bang $\left(10^{-43} \mathrm{sec}\right.$ or $\left.10^{32} \mathrm{GeV}\right)$, the quantum universe as a whole is chaotic, and the local quantum-chaotic graviton is active at the initial time of the creation of the universe $\left(10^{-44} \mathrm{sec}-10^{-35}\right.$ sec). When the temperature drops $\left(<10^{27} \mathrm{~K}\right.$ ), the symmetry of the grand unity is broken.

The pseudo-vacuum in the Big Bang of the universe is the vacuum of high density energy. The temperature of $10^{16} \mathrm{GeV}$ corresponds to the universe just created within $10^{-35} \mathrm{sec}$ in the Big Bang, when it was the size of grapefruit. It is further estimated that gravitation withdraws from the primitive unity when the universe is at $10^{-43}$ seconds and the temperature is $10^{32} \mathrm{~K}$. In the super high temperature, there are many boiling bubbles.

The universe boils at super-high temperature, and many vacuum bubbles appear, so the pseudo-vacuum decays into a real vacuum (the state of the lowest energy). With the decrease of temperature (freezing), the grand unified symmetry breaks ${ }^{[134]-[136]}$. Gravity, strong force and electroweak force successively separate from quantum gravity, super-symmetric unifying force and gauge field. A big rip-rebound cluster of quantum chaos-reticulate distribution emerges between the whole outwards-push of the universe and the local inwards-pull of gravity, strong force and electro-weak forces. At this moment, the universe forms a relatively complete ultra-synergy mechanism (system).

In this way, we can reveal the Higgs mechanism, the absence of magnetic monopoles and the inflation of the universe in a more ontological supernatural way.

For the cosmic ecosphere of the multi-world, the basic part of the unified research framework of nature evolution is summarized as the research on the research on the ultra-synergy physics of quantum Physic-Sphere. This research includes three levels: the research of spin-network ultra-synergy for the ecosphere of multi-world (research of quantum spatiotemporal evolution dynamics), the research of natural paradigm ultra-synergy for high-dimensional supergravity, and the research of quantum gravisphere ultra-synergy for the cosmic creation process. 
In the analysis of this series of papers, as a variable of spin network mode, spatiotemporal mode variable is a vector composed of a series of variables, which include: dimension, curvature, range, expansion rate, dynamics, quantization, distribution (inhomogeneity, concentration, fluctuation), synergy, etc.

Furthermore, we can regard space-time as a spin network system closely related to energy and mass, and the state variables of spin network system should include at least spin angular momentum, scale (range), dynamics, quantization (spin quantum number), magnetic moment, number of network nodes, distribution of network nodes (inhomogeneity, concentration, fluctuation), synergy (cooperation and competition), etc.

Now, by the ultra-synergy paradigm composed of spin network space, cosmic paradigm vector and cluster-inflating configuration, we explore to establish the basis of cosmic paradigm dynamics between our observable universe and the multi-verse world system (the unobservable world).

In theory, our observable universe is placed in the world system of multiverse. Although we don't know how big the world system of multiverse is, we can ideologically view the world system as the ultimate result without great any more. Compared with the world system of multi-verse, our observable universe is a "small open system". Especially the initial quantum universe can be seen as the movement of a micro system coupled with the world system without great any more (equivalent to a huge and incomparable "heat reservoir"). Since the world system as a heat reservoir is large enough and has many degrees of freedom, its internal relaxation process is very fast, and each degree of freedom is little affected by our quantum universe, it can be considered that the world system of multi-verse is basically in equilibrium. Because our observable quantum universe is very small (micro-system) relative to the Multi-verse world system, it is generally necessary to describe its motion behavior with quantum theory, which increases the complexity of the theory.

We discuss the paradigm dynamical behavior of our early quantum universe $U$ in the Multi-verse world system (large heat reservoir) $W$, which corresponds to the Heisenberg image. Hamiltonian of total system $W \oplus U$

$$
H_{T O L}=H_{W}+H_{U}+H_{U W}=H_{0}+H_{U W}
$$

here $H_{W}$ is the Hamiltonian of $W$ itself, $H_{U}$ is the Hamiltonian of $U$ itself.

The relevant Liouville operator can also be written as

$$
L_{T O L}=L_{W}+L_{U}+L_{U W}=L_{0}+L_{U W}
$$

All the mechanical quantities of the total system $W \oplus U$ constitute the $C^{*}$ algebra $u^{T O L}$.

We can take the cosmic paradigm vector as an extension of the cosmic paradigm and describe it from $S$ sides. Consider here: $u_{1}$. energy density, $u_{2}$. accumulation intensity, $u_{3}$. spatiotemporal dimension (high dimension, no upper limit, including fractal dimension), $u_{4}$. curvature, $u_{5}$. expansion rate (or contraction rate), $u_{6}$. inhomogeneity, $u_{7}$. concentration, $u_{8}$. fluctuation range, etc.

If the temperature of the multi-verse world system as a heat reservoir is $\beta^{-1}$, the inner product can be introduced into $u^{T O L}$ :

$$
\left(K_{1}, K_{2}\right)=\operatorname{Tr} K_{1}^{+} K_{2} \rho_{T O L, 0}, \quad K_{1}, K_{2} \in u^{T O L}
$$


here $\rho_{T O L, 0}=e^{-\beta H_{T O L}} / \operatorname{Tr} e^{-\beta H_{T O L}}$. By formula ( 267), we introduce the distance

$$
\left\|K_{1}-K_{2}\right\|=\sqrt{\left(K_{1}-K_{2}, K_{1}-K_{2}\right)}
$$

We can make $u^{T O L}$ complete and get the Hilbert space $\mathscr{H}_{\beta}{ }^{T O L}$. The total Liouville operator $L$ is Hermitian in $\mathscr{H}_{\beta}{ }^{T O L}$. What we really care about is the paradigm vectors of the early quantum universe $U$, which constitute a subspace $\mathscr{H}_{\beta}{ }^{U}$ of $\mathscr{H}_{\beta}{ }^{T O L}$. If the vector $\underline{e}=\left\{e_{i}\right\}_{i}$ is a set of bases in $\mathscr{H}_{\beta}{ }^{T O L}$, then the motion of the $U$ system is completely given by the temporal behavior of $\underline{e}(t)$.

Now, we introduce the projection operator:

$$
P A=(A, \underline{e}) \cdot(\underline{e} \cdot \underline{e})^{-1} \cdot \underline{e}, \quad A \in \mathscr{H}_{\beta}^{T O L}
$$

here $(\underline{e} \cdot \underline{e})^{-1}$ is the inverse matrix of dyadic $(\underline{e} \cdot \underline{e})$. After separating $P$ and $Q=1-P$ and solving $Q$ motion formally, it is not difficult to obtain the generalized Langevin equation satisfied by $\underline{e}(t)$ :

$$
\frac{d}{d t} \underline{e}(t)=i Q \cdot \underline{e}(t)-\int_{0}^{t} d \tau K(\tau) \cdot \underline{e}(t-\tau)+\underline{F}(t)
$$

here

$$
i Q=(\underline{\dot{e}} \cdot \underline{e}) \cdot(\underline{e} \cdot \underline{e})^{-1}, \quad \underline{F}(t)=e^{i Q L Q t} Q \underline{\dot{e}}(0), \quad K(\tau)=-i(\underline{F}(\tau) \underline{F}(0)) \cdot(\underline{e} \cdot \underline{e})^{-1}
$$

and $(\underline{F}(t) \underline{e}(0))=0, t>0,(268)$ is equivalent to the Liouville equation of the total system $W \oplus U$.

However, because the multi-verse world system as a heat reservoir is very large relative to our early quantum universe and has strong internal relaxation, its correlation function $K(\tau)$ decays rapidly with $\tau$ (because $Q \dot{e}$ is only a mechanical quantity of heat reservoir), or in other words, the transformation of the $\underline{e}(t)$ of the $U$ system within the decay time $\tau_{W}\left(t>\tau_{W}\right)$ of $K(\tau)$ is very small and Markov approximation can be used:

$$
\int_{0}^{t} K(t-\tau) \cdot \underline{e}(\tau) d \tau \sim \theta \cdot \underline{e}(t)
$$

here $\theta=\int_{0}^{\infty} K(\tau) d \tau$, thus Langevin equation is obtained:

$$
\frac{d}{d t} \underline{e}(t)=(i Q-\theta) \cdot \underline{e}(t)+\underline{F}(t)
$$

$\underline{F}(t)$ is the fluctuating force and $\theta$ is the generalized friction coefficient (dissipation coefficient). Taking the limit $\tau_{W} \rightarrow 0$, the correlation function of $\underline{F}(t)$ has white spectrum, and $K(\tau)$ can be rewritten as follows

$$
K(\tau)=-i(\underline{F}(t) \underline{F}(0)) \cdot(\underline{e} \cdot \underline{e})^{-1}=\theta \delta(t)
$$

This formula directly relates the friction coefficient $\theta$ in Markov process ( 270 ) to the fluctuation force $\underline{F}$. In other words, it links the dissipation coefficient of our early quantum universe $U$ with the motion fluctuation of the Multi-verse world system as a heat reservoir, and introduces statistical factors (Markov approximation) in the derivation process.

In fact, describing the open quantum universe (system) with the generalized Langevin equation 
( 270 ) is to divide the forces acting on the quantum universe by the Multi-verse world system as a heat reservoir into two parts: one is the average force, i.e. the generalized friction force $-\theta \cdot \underline{e}(t)$, and the other is the random force $\underline{F}$ (the average is zero). When the distinction (actually the projection operator $P$ ) between the quantum universe as an open system and the multi-verse world system as a heat reservoir has been established, this division of the forces of the multi-world system on our quantum universe is unique. However, for a specific problem, how to distinguish the quantum universe at a later time ( $t>0$ ) has changed. Generally speaking, the choice of projection operator $P$ is different, and the results are different. In the above derivation, the choice of different $P$ is expressed as the definition of different inner product. We take as ( 267 )

$$
\left(K_{1}, K_{2}\right)=\operatorname{Tr} K_{1}^{\dagger} K_{2} \rho_{T O L, 0}
$$

Even though $K_{1}$ and $K_{2}$ are only mechanical quantities of the quantum universe $U,\left(K_{1}, K_{2}\right)$ is related to the multi-verse world system $W$ by $H_{U W}$ in $H_{T O L}$. Specific calculations ( 267 ) will be difficult. If

$$
\left(K_{1}, K_{2}\right)=\operatorname{Tr} K_{1}^{\dagger} K_{2} \rho_{0}
$$

here $\rho_{, 0}=e^{-\beta H_{0}} / T r e^{-\beta H_{0}}$, then, the concrete calculation will be much simpler; however, the Liouville operator $L$ is no longer Hermitian operator under the definition of this inner product, so it should be taken in the derivation ( 268 )

$$
(L Q \underline{F}(\tau), \underline{e})=\left(\underline{F}(\tau), Q L^{\dagger} \underline{e}\right)=i\left(\underline{F}(\tau), \underline{F}^{\dagger}\right)
$$

here $\underline{F}^{\dagger}=-i Q L^{\dagger} \underline{e}$. When the Markov limit is taken, the obtained correlation function is

$$
K(\tau)=-i\left(\underline{F}(\tau) \underline{F}(0)^{\dagger}\right) \cdot(\underline{e} \cdot \underline{e})^{-1}=\theta \delta(\tau)
$$

For the case of real fluctuation force (in many classical cases, $\underline{F}$ is a real function), ( 273 ) is the same as ( 271 ), and the fluctuation dissipation relationship remains unchanged. However, if $\underline{F}(t)$ is complex, then ( 273 ) is different from ( 271 ). As long as $\underline{e}$ is Hermitian, we can think that ( 273 ) is to satisfy the fluctuation dissipation relationship. So, in most cases, the inner product is defined by ( 272 ). If $\underline{e}$ is taken as a set of orthogonal normalized complete bases, Langevin equation can be obtained.

\section{Ultra-Synergy Mechanism between Particle and Universe}

We propose to incorporate Higgs mechanism, Fermi field and Yukawa coupling into the ultrasynergy system between particles and the universe, and to use Higgs mechanism as the basic link of the ultra-synergistic mechanism system, so as to enhance the explanatory power of Higgs mechanism.

By the existing observation, exploration and research, the physical process of the very early universe took place in the very short instant. Therefore, people can not go back to that period to directly "observe" the evolution of the universe, but can only reconstruct the correlation function of quantum perturbation in that period by indirect method ${ }^{[137]-[138]}$. Regrettably, it is impossible to distinguish whether the universe was expanding or contracting at that time in principle only by correlation functions. So there is no definite way to distinguish early cosmic inflation from other alternative 
models by observing it. In any case, in terms of naturalness and simplicity, inflation cosmology is the simplest and most early natural cosmological model compared with those alternative models.

For the cosmic ecosphere of the multi-verse world, this series sets up a larger theoretical framework at a deeper level, which combines the creation of the universe with the unification of basic interactions. On the one hand, we re-study the primitive unification of fundamental interactions under the extreme conditions of the creation of the universe; on the other hand, we re-study the creation of the universe on the basis of the primitive unification of fundamental interactions.

In order to reduce the excessive dependence of various inflationary universe models on the initial conditions, we will fully consider and study a great impetus, which is a super-synergistic dynamic system between particles and the universe. Through this great impetus, we can reduce the excessive dependence of the inflationary universe model on the initial conditions to a large extent, and increase the reasonable dependence of the inflationary universe model on the evolution process to a large extent.

Combining physical geometry with geometric physics in the supernatural way of ontology, and then combining physical cosmology with cosmic physics, we study the ultra-synergy (emergence of big rip-rebound clusters with quantum chaotic-reticulate distribution) between the universe and ever-increasing particles (clusters), especially the ultra-synergy (emergence of big rip-rebound clusters with quantum chaotic-reticulate distribution) between the quantum universe in its Big Bang and ever-increasing quantum gravi-sources (clusters). Then we study the ultra-synergy (emergence of big rip-rebound clusters with quantum chaotic-reticulate distribution) between the universe in its inflation and ever-increasing initial gravi-resours (clusters) and initial strong force sources (clusters), especially the ultra-synergy (emergence of big rip-rebound clusters with quantum chaotic-reticulate distribution) between the universe in its expansion and ever-increasing gravi-sources (clusters), strong force sources (clusters) and electro-weak force sources (clusters).

As shown in Fig. 1 (Li Zongcheng, 2019), each initial strong force source of high-intensity forms a rip-rebound center for the inflation or expansion of the universe at the microscopic level. Here the dark part in the figure represents a larger density, while the light part represents a smaller density.

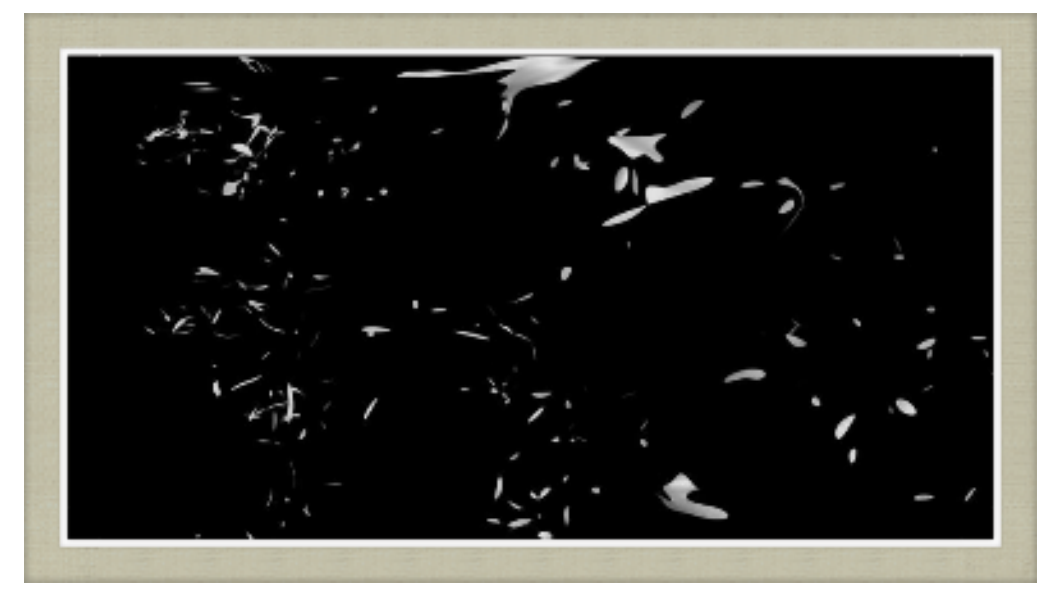

Fig. 1 Each initial strong force source of high-intensity forms a rip-rebound center for the inflation or expansion of the universe at the microscopic level 
As shown in Fig. 2 (Li Zongcheng, 2019), each initial electro-weak force source of high-intensity forms a rip-rebound center for the inflation or expansion of the universe at the microscopic level. Here the dark part in the figure represents a larger density, while the light part represents a smaller density.

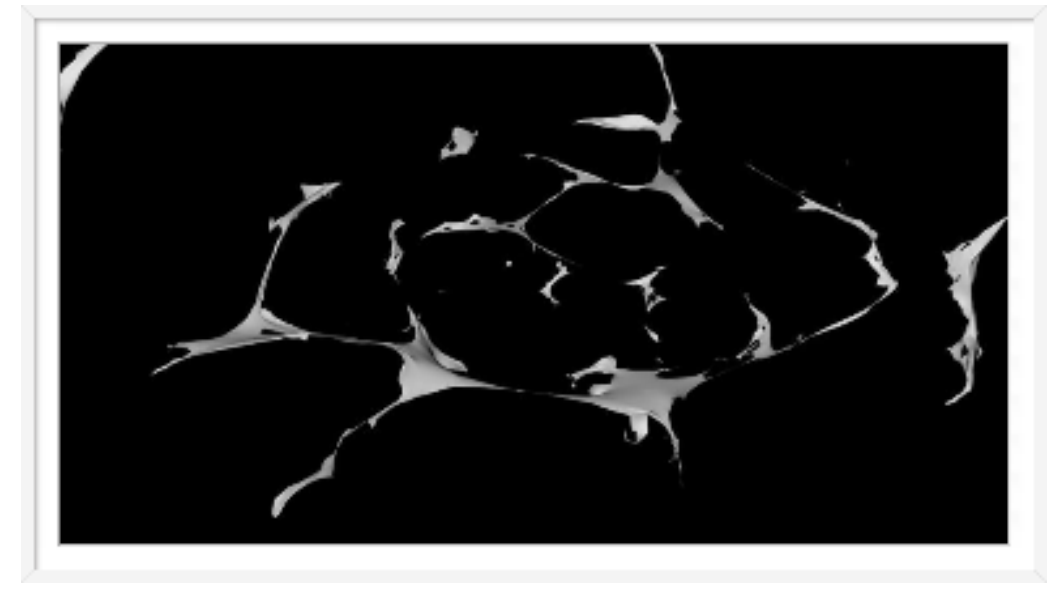

Fig. 2 Each initial electro-weak force source of high-intensity forms a rip-rebound center for the inflation or expansion of the universe at the microscopic level

We further find that at the initial moment for fundamental forces to separate, both the quantum gravi-source and the gravi-source, as well as the strong force source and the electro-weak force source, are very powerful, and between ever-increasing interaction sources there should form superimposed amplification effect. These ultra-synergy in several phase transitions during the creation of the universe are the big impetus for the rip-rebound- inflation and further expansion of the universe.

According to the calculation, there are about $10^{80}$ particles in the universe, the effective range of electromagnetic interaction $>10^{-16} \mathrm{~cm}$, the radius of nucleon is $0.8 \times 10^{-13} \mathrm{~cm}$, the radius of electron is $r_{e}=e^{2} / m_{e} c^{2}=(2.8179380 \pm 0.000007) \times 10^{-17} \mathrm{~cm}$, and the mass of electron is $m_{e}=(9$. $109534 \pm 0.000047) \times 10^{-34} \mathrm{~g}$, which is about $1 / 1836$ of proton mass.

In quantum theory, any substance is regarded as a combination of oscillations in a quantum field. These oscillations are transmitted in the form of "packets" in the field, so-called "quantum", which can be regarded as a kind of particle. Here there are two different kinds of fields: the material field containing matter particles and the force field acting as the medium of action. The Higgs particle is also an oscillation of the field, which is usually referred to by physicists as the "Higgs field" [139]-[140].

For the ultra-synergy of the early universe, the Higgs field is a necessary basic joint. We know that the standard model of particle physics can not be separated from the Higgs field, because the standard model can only be established under the assumption that particles have no mass. For example, in electromagnetic force, the medium of transmission is photons without mass, which is no problem. However, the medium of transmission of weak nuclear force is three kinds of particles with mass: two charged $W$ particles and one $Z$ particle, which are essentially different from photons with zero mass, thus threatening the standard model. Later, Franois Englert, Robert Brout and Peter Higgs, et al., supplemented in time and proposed an idea to eliminate this threat, giving the way for particles to obtain mass ${ }^{[25][141][142]}$. 
With regard to the super-inflating universe in the big impetus, we can put forward the following basic ideas here: In the very early universe, there was not only an accelerated expansion stage $(\ddot{R}>0)$, with an exponential growth $R \propto e^{H t}$ of the scale factor of the universe, in which $H$ was the Hubble constant; but also a big impetus stage from super-synergy (represented by the emergence of big rip-rebound clusters of quantum chaos-reticulate distribution), which firstly forcibly restrained the cosmic boom, then sped up beyond the limit, and then quickly turned to flash deceleration. For such a strong rip-rebound-inflation under a big impetus, we need to introduce a ultra- synergy scalar field $\phi_{B S}$, which satisfies the minimum coupling conditions with repulsion and gravity.

Figure 11 (Li Zongcheng, 2019) shows the high-dimensional supergravity, quantum gravity and four fundamental interactions in the creation of the universe.

The energy density and pressure of the ultra-synergistic scalar field can be expressed as follows:

$$
\rho_{\text {ultra-syn }}=\frac{1}{2} \dot{\phi}_{B S}^{2}+(1-A) V\left(\phi_{B S}\right), \quad p_{u l t r a-s y n}=\frac{1}{2} \dot{\phi}_{B S}^{2}-(1-A) V\left(\phi_{B S}\right),
$$

here $V\left(\phi_{B S}\right)$ is the potential energy of ultra-synergy field, $A=-\ddot{R} / R H^{2}, A$ is a binary quantity:

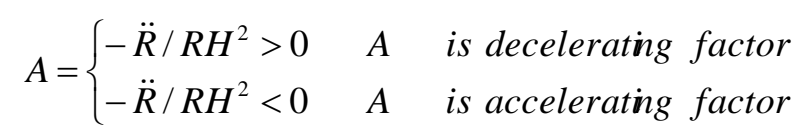

Expression (1) can be written in the following form:

$$
\begin{aligned}
\rho_{\text {ultra-syn }}=N^{b}\left\{\left(1+\lambda_{d-e n}\right)\left[\frac{1}{2} \dot{\phi}_{\text {repl }}^{2}+\left(1-A_{\text {acce }}\right) V\left(\phi_{\text {repl }}\right)\right]\right. \\
\left.-\left(1+\lambda_{d-\text { matt }}\right)\left[\frac{1}{2} \dot{\phi}_{\text {grav }}^{2}+\left(1-A_{\text {dece }}\right) V\left(\phi_{\text {grav }}\right)\right]\right\}
\end{aligned}
$$

a)

$$
\begin{aligned}
p_{\text {ultra-syn }}=N^{b}\left\{\left(1+\lambda_{d-e n}\right)\left[\frac{1}{2} \dot{\phi}_{\text {repl }}^{2}-\left(1-A_{\text {acce }}\right) V\left(\phi_{\text {repl }}\right)\right]\right. \\
\left.\quad-\left(1+\lambda_{d-\text { matt }}\right)\left[\frac{1}{2} \dot{\phi}_{\text {grav }}^{2}-\left(1-A_{\text {dece }}\right) V\left(\phi_{\text {grav }}\right)\right]\right\}
\end{aligned}
$$

b)

here $N$ is the total number of gravi-sources, $b$ is the super-inflation index, $\phi_{\text {repl }}$ is the scalar field under the repulsion of the universe, $\phi_{\text {grav }}$ is the scalar field under the quantum gravity, $A_{\text {acce }}$ is accelerating factor, $A_{\text {dece }}$ is decelerating factor, $\lambda_{\text {d-en }}$ is the enhancement coefficient of dark energy, $\lambda_{d \text {-matt }}$ is the enhancement coefficient of dark matter, and the first term of the full expansion ( $276 a$ ) represents the energy density under the repulsion of the whole universe to $N^{b}$ gravi-sources, and the second term represents the energy density under the gravitation of the arche-quantum to $N^{b}$ gravi-sources, the first term of the full expansion ( $276 b$ ) represents the pressure under the repulsion of the whole universe to $N^{b}$ gravi-sources, and the second term represents the pressure under the repulsion of the whole universe to $N^{b}$ gravi-sources, and the second term represents under the gravitation of the arche-quantum to $N^{b}$ 
gravi-sources.

The extended form of Klein-Gorden equation for ultra-synergy scalar field should be

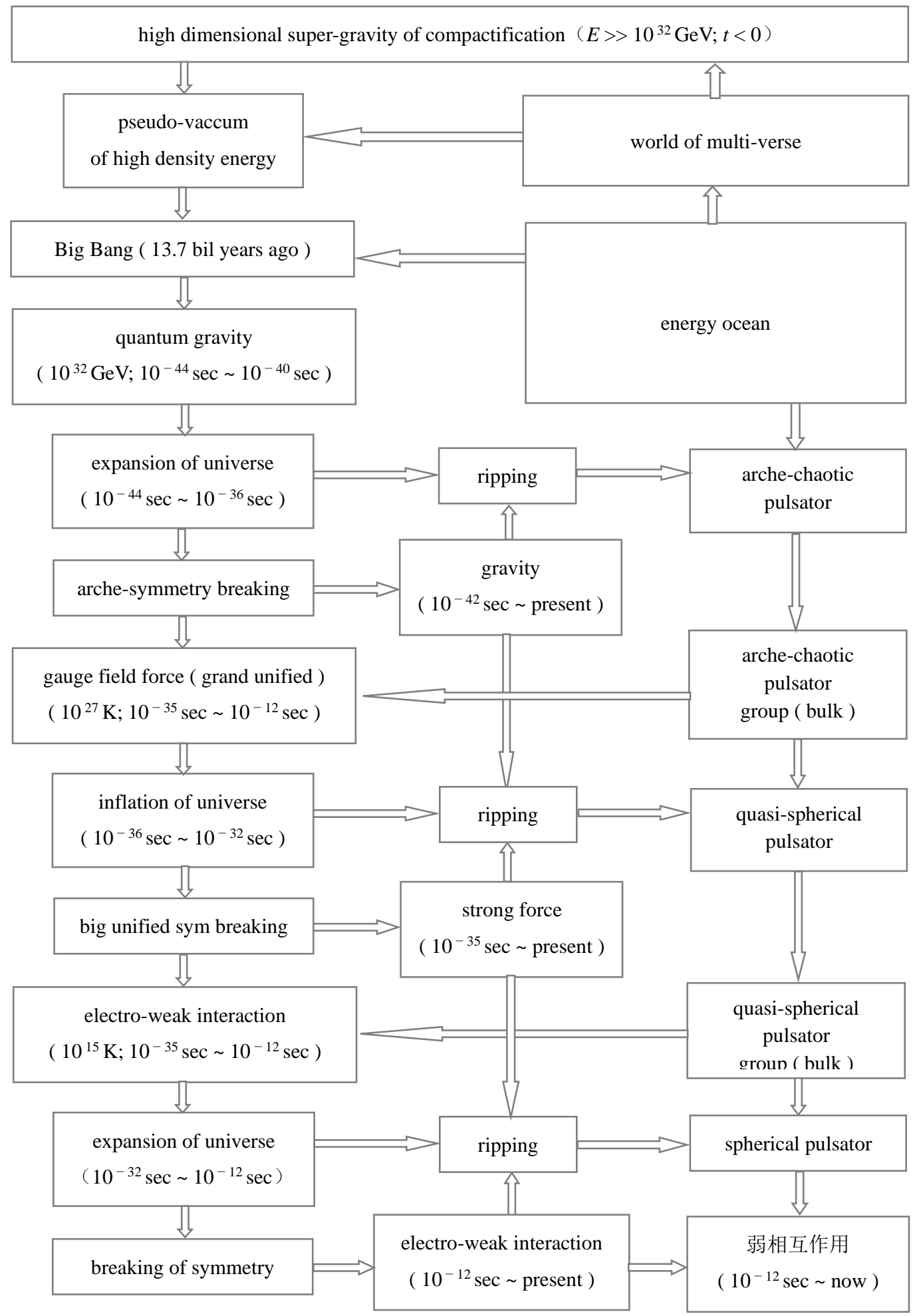

Figure 11 High-dimensional supergravity, quantum gravity and four fundamental interactions 


$$
\ddot{\phi}_{B S}+3 H \dot{\phi}_{B S}+(1-A) \frac{d V}{d \phi_{B S}}=0
$$

Assuming that the ultra-synergy scalar field is dominant in all components during the rip-reboundinflation stage of the big impetus, the extended form of Freeman equation should be

$$
H^{2}=\frac{8 \pi G}{3}\left[\frac{1}{2} \dot{\phi}_{B S}^{2}+(1-A) V\left(\phi_{B S}\right)\right]
$$

Expression ( 278 ) can be further written in the following form:

$$
\begin{array}{r}
H_{u-s y n}^{2}=N^{b}\left\{\left(1+\lambda_{d-e n}\right) \frac{8 \pi G}{3}\left[\frac{1}{2} \dot{\phi}_{\text {repl }}^{2}+\left(1-A_{\text {acce }}\right) V\left(\phi_{\text {repl }}\right)\right]\right. \\
\left.-\left(1+\lambda_{d-\text { matt }}\right) \frac{8 \pi G}{3}\left[\frac{1}{2} \dot{\phi}_{\text {grav }}^{2}+\left(1-A_{\text {dece }}\right) V\left(\phi_{\text {grav }}\right)\right]\right\}
\end{array}
$$

Obviously, when $\dot{\phi}_{B S}^{2}<<V\left(\phi_{B S}\right)$ (slow roll approximation), it can be seen from the formulas ( 277 ) and ( 278 ), which can provide super accelerated expansion. Because $\dot{\phi}_{B S}^{2}$ is very small, the extended forms of Friedmann equation and Klein-Gorden equation are approximated to

$$
H^{2}=(1-A) \frac{8 \pi G}{3} V(\phi), \quad \dot{\phi} \approx-(1-A) \frac{1}{3 H} \frac{d V}{d \phi} .
$$

Because $d V / d \phi_{B S}$ is very small and $H$ is near constant, $a(t)$ increases exponentially.

Based on the super-synergy mechanism with the great impetus, we can infer that in the extreme background and environmental conditions of the universe, through the big rip-rebound cluster of quantum chaos-reticulate distribution emerged between the huge outwards-push (the Big Bang force, the inflationary force and the expansive force) and the huge inwards-pull (gravitation, strong force, electromagnetic force and weak force), the basic unit of matter must have undergone three basic forms successively: complete chaotic pulsator, quasi-spherical pulsator and spherical pulsator. Various pulse modes of the quantum gravi-pulsator give rise to a variety of elementary particles. As the smallest condensation of energy, the quantum gravi-pulsator is mainly manifested in the $10^{-44} \mathrm{sec}-10^{-35} \mathrm{sec}$ of the creation of the universe. It is neither a punctiform particle nor a superstring, supermembrane or superembryo.

According to the quantum arche-pulsator theory established in this series, the elementary particles in the standard model are actually some quantum pulsators (including chaotic pulsator, quasi-spherical pulsator and spherical pulsator). The different pulsations and motions of the quantum arche-particle can produce different kinds of basic particles. All the most basic particles, including positive and negative quarks, electrons, neutrinos and so on, as well as the four fundamental forces "particles" (strong and weak force particles, electromagnetic force particles, and gravitational particles), are derived from the pulsating quantum arche-particle, and the difference between the various particles lies only in the way and shape of the quantum arche-pulsation. Quantum arche-pulsators also contain fermions, one of the 
elementary particles of matter. All particles can be obtained by the different pulsations and motions of the quantum arche-particles. Essentially, all particles are quantum pulsators.

In the super-inflatiing universe model established in this series, Higgs field becomes the origin of vacuum energy in quantum mechanics and then the origin of stationary mass under the big impetus of super-synergy. After the end of strong ripping, strong rebound and strong inflation, the super-synergy scalar field recedes and produces matter and radiation. The radiation from recession begins to re-heat the universe, followed by the standard Big Bang universe.

There are still many problems with the standard model. According to the standard model, neutrinos should have no mass, but later studies have found that such particles do seem to have mass. In addition, the standard model can only describe visible matter, which accounts for only about a fifth of the total amount of matter in the universe. We still know little about the nature of mysterious dark matter.

We can't directly observe dark matter, but we can perceive its existence through its gravitational action $^{[137][143]}$. It is the gravitational pull of dark matter that prevents galaxies from disintegrating in the universe. Dark matter hardly reacts with visible matter at all. However, we now know that Higgs particles are unique. Perhaps through the coupling of Higgs mechanism, Fermion field and Tangkawa, we can find super-synergy between particles and the universe, which will help us to establish some kind of connection between these two completely different substances.

According to the explanation of the Higgs mechanism, it is assumed that there exists a scalar field called the Higgs field throughout the universe. By coupling with the Higgs field, some particles without mass can obtain energy. In the mass-energy relationship, this is equal to obtaining mass. The stronger the coupling between the particle and the Higgs field is, the larger the mass of the particle is. If the Higgs field does not exist, the mass of quarks, $W$ bosons and $Z$ bosons will all become zero. Because only about $1 \%$ of the mass of composite particles such as protons and neutrons is attributed to the quarks they contain, their properties will only change slightly. The mass of tau $(\tau)$ and muon $(\mu)$ will also become zero, but they have nothing to do with real life. Only when the mass of electrons becomes zero will it have a great impact on the world. The smaller the mass of the electron is, the larger the size of the atom is. When the mass of electrons becomes zero, super-large atoms will be dismantled by collision. All nuclei and electrons will mix together. Atoms cannot exist alone, nor will there be water, air and the world in which human beings live.

Higgs field can break symmetry ${ }^{[137][138][143]}$. Without the Higgs field, all charged leptons, i.e., electrons, tau $(\tau)$ and muon $(\mu)$, would become the same, because their differentiated masses would all be zero. Similarly, quarks with charges of $+2 / 3$, i.e., upper quarks, odd quarks and top quarks, will become the same, while quarks with charges of $-1 / 3$, i.e., lower quarks, charm quarks and bottom quarks, will become the same.

Some cosmologists believe that the Higgs field is the origin of vacuum energy ${ }^{[25][140][141]}$. At the very beginning of the universe, when the temperature is very high, the symmetry of the Higgs field has no characteristic, and the energy of the universe is no different. As the temperature of the universe decreases, the symmetry is broken by several successive phase transitions, which give rise to the ever-changing universe. The symmetry breakage caused by the last phase transition breaks the electroweak force and separates the weak force from the electromagnetic force. At present, physicists have been able to make experiments to achieve the required conditions for the phase transition, but the 
conditions for separating the phase transition of electro-weak and strong forces are still far from achievable. In any case, the Higgs field, recognized as the origin of static mass, is also the key to the study of strong forces.

The super-synergy (the emergence of big rip-rebound clusters with quantum chaotic-reticulate distribution) emerged between the Big Bang of the quantum universe and the ever-increasing quantum gravi-sources (clusters) during the creation of the universe can be used to explain the magnetic monopole problem. The magnetic monopole problem involves the Big Bang theory and the grand unified theory, sometimes referred to as the "exotic-relics problem". The grand unified theory proposes that if the temperature of the early universe exceeds the grand unified temperature (about $10^{32} \mathrm{~K}>10^{27}$ $\mathrm{K}$ ), the electromagnetic force, the strong nuclear force and the weak nuclear force will be unified into a "grand unified force". Because of the expansion of the universe, the temperature will continue to decrease. When the temperature is lower than the grand unified temperature, the spontaneous symmetry breakage will occur, and the physical properties of the weak force and the strong nuclear force will begin to become different, so the phase transition will occur.

Phase change due to the breaking of symmetry usually results in "topological defects" [137][138][143]. The grand unified theory predicts that the grand unified phase transition will produce a zerodimensional punctiform topological defect whose physical properties are like magnetic monopoles. The grand unified theory predicts that the grand unified phase transition will not only produce this kind of magnetic monopole, but also remain to this day, even possibly become the main component of the universe due to its extremely stable state. However, today's universe is not full of magnetic monopoles. Scientists have never even found any magnetic monopoles, which sets a very low upper limit for the density of magnetic monopoles in the universe ${ }^{[137][138][143]}$. The theory of the great impetus with big rip-rebound- inflaiton mechanism, not only shows that the rapid expansion of the universe will cause the density of magnetic monopoles to drop sharply in space to an order of magnitude that can not be detected by current instruments, but also implies that a large number of magnetic monopoles should be destroyed in the big rip-rebound cluster with quantum chaotic-reticulate distribution emerged under the ultra-synergy.

\section{Equation of Quantum-Classic Mechanics for Complexity}

On the basis of the above basic hypotheses and its inferences, we investigate to create the basic equations of quantum-classical mechanics for space-time complexity, by the Wigner distribution as nonlinear time-frequency transform. From the perspective of information, the basic equations of quantum-classical mechanics (the following formulas ( 290 )) are a scheme of the full- view treatment for the classical dynamic information and quantum dynamic information.

For any quantum system, an operable model of the "environment" is the set of a large number of oscillators, or equivalently represented by scalar fields. The displacement coordinate (position) of particles is denoted as $x$, it interacts with the scalar field $\varphi(x, t)$ spreading in direction $y$ : $H_{\text {int }}=\varepsilon x \frac{d \varphi}{d t}$; the order change (such as density difference, entropy change, information entropy change) coordinate of particles is $\sigma$, it interacts with the scalar field $\varphi(x, t)$ spreading in direction $y$ : 
$H_{\text {int }}=\pi \sigma \frac{d \varphi}{d t}$. For the thermal excitation of scalar fields under high temperature, we can set the master equation of particles satisfied by the particle density matrix $\rho\left(x, x^{\prime}\right)$ in the expression of $x$ :

$$
\frac{d \rho_{x}}{d t}=-\frac{i}{\hbar}\left[H, \rho_{x}\right]-\ell\left(x-x^{\prime}\right)\left(\frac{d \rho_{x}}{d x}-\frac{d \rho_{x}}{d x^{\prime}}\right)-\frac{2 m \ell k T}{\hbar^{2}}\left(x-x^{\prime}\right)^{2} \rho_{x},
$$

and put forth the master equation of particles satisfied by the particle density matrix $\rho\left(\sigma, \sigma^{\prime}\right)$ in the expression of $\sigma$ :

$$
\frac{d \rho_{\sigma}}{d t}=-\frac{i}{\hbar}\left[H, \rho_{\sigma}\right]-\mu\left(\sigma-\sigma^{\prime}\right)\left(\frac{d \rho_{\sigma}}{d \sigma}-\frac{d \rho_{\sigma}}{d \sigma^{\prime}}\right)-\frac{2 m \mu k T}{\hbar^{2}}\left(\sigma-\sigma^{\prime}\right)^{2} \rho_{\sigma},
$$

where, $H$ is the Hamilton quantity of particles, $l$ and $\mu$ are relaxation rates, and $T$ is the temperature of the field $\varphi$. On the one hand, $T$ relates to viscosity coefficient $\eta_{x}$, while $\eta_{x}$ depends on the coupling constant $\varepsilon$ of particles and fields in $H_{\text {int }}$, i.e. $l=\eta_{x} / 2 m, \eta_{x}=\varepsilon^{2} / 2$; on the other hand, relates to viscosity coefficient $\eta_{\sigma}$, while $\eta_{\sigma}$ depends on the coupling constant $\pi$ of particles and fields in $H_{\text {int }}$, i.e. $\mu=\eta_{\sigma} / 2 m, \eta_{\sigma}=\pi^{2} / 2$. In equation ( 281 ), the first item is the kinetic joint-varying of motion-like, and it is the inference of Schrodinger equation. In equation ( 282 ), the first item is the kinetic joint-varying of evolution-like, and it is the extension of Schrodinger equation. The second item is the dissipation effect of the environment, and the third item is the fluctuation causing the Brown motion, which is the root cause of the decoherence.

By $\dot{\rho}_{\gamma}=\frac{\partial \rho_{\gamma}}{\partial \rho_{x}} \dot{\rho}_{x}+\frac{\partial \rho_{\gamma}}{\partial \rho_{\sigma}} \dot{\rho}_{\sigma}$, with ( 281 ) and ( 282 ), we can create the master equation of particles satisfied by the particle density matrix $\rho\left(\gamma, \gamma^{\prime}\right)$ in the expression of $\gamma$ :

$$
\begin{aligned}
\frac{d \rho_{\gamma}}{d t} & =\frac{\partial \rho_{\gamma}}{\partial \rho_{x}} \frac{d}{d t}\left\{-\frac{i}{\hbar}\left[H, \rho_{x}\right]-\ell\left(x-x^{\prime}\right)\left(\frac{d \rho_{x}}{d x}-\frac{d \rho_{x}}{d x^{\prime}}\right)-\frac{2 m \ell k T}{\hbar^{2}}\left(x-x^{\prime}\right)^{2} \rho_{x}\right\} \\
& +\frac{\partial \rho_{\gamma}}{\partial \rho_{\sigma}} \frac{d}{d t}\left\{-\frac{i}{\hbar}\left[H, \rho_{\sigma}\right]-\mu\left(\sigma-\sigma^{\prime}\right)\left(\frac{d \rho_{\sigma}}{d \sigma}-\frac{d \rho_{\sigma}}{d \sigma^{\prime}}\right)-\frac{2 m \mu k T}{\hbar^{2}}\left(\sigma-\sigma^{\prime}\right)^{2} \rho_{\sigma}\right\}
\end{aligned}
$$

The coherent super-positions of two Gauss wave packets are considered:

$$
\chi(x)=\chi^{+}(x)+\chi^{-}(x), \quad \theta(\sigma)=\theta^{+}(\sigma)+\theta^{-}(\sigma),
$$

where the widths of wave packet are $\delta_{x}$ and $\delta_{\sigma}$, the distance that they are away from the center are respectively $\Delta x$ and $\Delta \sigma, \Delta x<<\delta_{x}, \Delta \sigma<<\delta_{\sigma}$. There are 4 peaks on plane $\left(x, x^{\prime}\right)$ in the density matrix $\rho_{x}\left(x, x^{\prime}\right)=\chi(x) \chi^{*}\left(x^{\prime}\right)$, where two come from the diagonal element (the position $x=x^{\prime}$ of the peak), the other two are from the non diagonal element $\left(x=-x^{\prime}\right)$. The last item of master equation ( 281$)$ is proportional to $\left(x-x^{\prime}\right)^{2}$. It has little effect on the diagonal element, but on the non diagonal element becomes the factor causing the attenuation. The decay rate can be written directly from the equation: $\tau_{D, x}^{-1} \approx 2 \ell \frac{m k T(\Delta x)^{2}}{\hbar^{2}}, \tau_{D, \sigma}^{-1} \approx 2 \mu \frac{m k T(\Delta \sigma)^{2}}{\hbar^{2}}$. Decoherence time $\tau_{D, x}$ and $\tau_{D, \sigma}$ are respectively 


$$
\begin{aligned}
\tau_{D, x}=\tau_{R, x}\left(\frac{\lambda_{T}}{\Delta x}\right)^{2} \text {, and } \tau_{D, \sigma} & =\tau_{R, \sigma}\left(\frac{\lambda_{T}}{\Delta \sigma}\right)^{2} \\
\tau_{D, x} \approx \tau_{R, x} \frac{\hbar^{2}}{2 \ell m k T(\Delta x)^{2}} & =\tau_{R, x}\left(\frac{\lambda_{T}}{\Delta x}\right)^{2}, \quad \tau_{D, \sigma} \approx \tau_{R, \sigma} \frac{\hbar^{2}}{2 \mu m k T(\Delta \sigma)^{2}}=\tau_{R, \sigma}\left(\frac{\lambda_{T}}{\Delta \sigma}\right)^{2} .
\end{aligned}
$$

It is known that quantum mechanics is expressed in Hilbert spaces, and classical mechanics is expressed in phase space. We can use the Wigner transform of wave function, set the following relation between the quantum dynamics and its classical limit :

$$
\begin{aligned}
& W_{x}(x, p)=\frac{1}{2 \pi \hbar} \int_{-\infty}^{\infty} e^{i p y / \hbar} \psi_{x}^{*}\left(x+\frac{y}{2}\right) \psi_{x}\left(x-\frac{y}{2}\right) d y, \\
& W_{\sigma}(\sigma, p)=\frac{1}{2 \pi \hbar} \int_{-\infty}^{\infty} e^{i p y / \hbar} \psi_{\sigma}^{*}\left(\sigma+\frac{y}{2}\right) \psi_{\sigma}\left(\sigma-\frac{y}{2}\right) d y .
\end{aligned}
$$

Wigner distributions $W_{x}(x, p)$ and $W_{\sigma}(\sigma, p)$ are real function, but may be negative.

Give the Fourier transform of $\psi_{x}: \varphi_{x}(p)=\frac{1}{\sqrt{2 \pi \hbar}} \int_{-\infty}^{\infty} e^{-i p \xi_{x} / \hbar} \psi_{x}\left(\xi_{x}\right) d \xi_{x}$, the distribution of $p$ is

$$
\varphi_{x}^{*}(p) \varphi_{x}(p)=\frac{1}{2 \pi \hbar} \int_{-\infty}^{\infty} e^{i p\left(\lambda_{x}-\xi_{x}\right) / \hbar} \psi_{x}^{*}\left(\lambda_{x}\right) \psi_{x}\left(\xi_{x}\right) d \xi_{x} d \lambda_{x} .
$$

Let $\lambda_{x}=x+\frac{y}{2}, \xi_{x}=x-\frac{y}{2}$, there is

$$
\varphi_{x}^{*}(p) \varphi_{x}(p)=\frac{1}{2 \pi \hbar} \int_{-\infty}^{\infty} e^{i p y / \hbar} \psi_{x}^{*}\left(x+\frac{y}{2}\right) \psi_{x}\left(x-\frac{y}{2}\right) d x d y=\int_{-\infty}^{\infty} W_{x}(x, p) d x,
$$

i.e. the integral of $W_{x}(x, p)$ to $x$ gives the distribution of $p$.

Give the Fourier transform of $\psi_{\sigma}: \varphi_{\sigma}(p)=\frac{1}{\sqrt{2 \pi \hbar}} \int_{-\infty}^{\infty} e^{-i p \xi_{\sigma} / \hbar} \psi_{\sigma}\left(\xi_{\sigma}\right) d \xi_{\sigma}$, the distribution of $p$ is

$$
\varphi_{\sigma}^{*}(p) \varphi_{\sigma}(p)=\frac{1}{2 \pi \hbar} \int_{-\infty}^{\infty} e^{i p\left(\lambda_{\sigma}-\xi_{\sigma}\right) / \hbar} \psi_{\sigma}^{*}\left(\lambda_{\sigma}\right) \psi_{\sigma}\left(\xi_{\sigma}\right) d \xi_{\sigma} d \lambda_{\sigma} \text { 。 }
$$

Let $\lambda_{\sigma}=x+\frac{y}{2}, \quad \xi_{\sigma}=x-\frac{y}{2}$, there is

$$
\varphi_{\sigma}^{*}(p) \varphi_{\sigma}(p)=\frac{1}{2 \pi \hbar} \int_{-\infty}^{\infty} e^{i p y / \hbar} \psi_{\sigma}^{*}\left(\sigma+\frac{y}{2}\right) \psi_{\sigma}\left(\sigma-\frac{y}{2}\right) d \sigma d y=\int_{-\infty}^{\infty} W_{\sigma}(\sigma, p) d \sigma,
$$

i.e. the integral of $W_{\sigma}(\sigma, p)$ to $\sigma$ gives the distribution of $p$.

For the least uncertain covariant wave packet, the Wigner distribution function is

$$
W_{\gamma}(\gamma, p)=\frac{1}{\pi \hbar} \exp \left[-\frac{\left(\gamma-\gamma_{0}\right)^{2}}{\delta^{2}}-\frac{\left(p-p_{0}\right)^{2} \delta^{2}}{\hbar^{2}}\right],
$$


here, $\gamma$ and $p$ obey Gauss distribution and satisfy the minimum uncertainty relation, which shows that $\psi_{\gamma}$ is the most approximate simulation of classical covariant particles (a point in the phase space) which can be given by the wave function of quantum mechanics (Hilbert space vector).

Wigner joint-distribution can be extended to the density matrix

$$
W_{\gamma}(\gamma, p)=\frac{1}{2 \pi \hbar} \int_{-\infty}^{\infty} e^{i p y / \hbar} \rho_{\gamma}\left(\gamma-\frac{y}{2}, \gamma+\frac{y}{2}\right) d y .
$$

For the coherent superposition $\chi(x)=\chi^{+(x)}+\chi^{-(x)}$ and $\theta(\sigma)=\theta^{+(\sigma)}+\theta^{-(\sigma)}$ of Gauss wave packet given by formula ( 284 ), their Wigner joint-distribution is

$$
W_{\gamma} \approx \frac{W_{\gamma}^{+}+W_{\gamma}^{-}}{2}+\frac{1}{\pi \hbar} \exp \left[-\frac{1}{\hbar^{2}} p^{2} \delta^{2}-\frac{\gamma^{2}}{\delta^{2}}\right] \cos \left(p \frac{\Delta \gamma}{\hbar}\right)
$$

here $W_{\gamma}^{+}$and $W_{\gamma}^{-}$are the Wigner joint-distribution of wave packets. The joint-varying equation (as the basic equation of quantum complexity mechanics for space-time complexity) of $W_{\gamma}$ can be given by the master equation ( 283$)$ for the density matrix $\rho_{\gamma}\left(\gamma, \gamma^{\prime}\right)$ of particles to meet :

$$
\begin{aligned}
\frac{d W_{\gamma}}{d t} & =\frac{\partial W_{\gamma}}{\partial W_{x}} \frac{d}{d t} \int d t\left\{\left(-\frac{p}{m} \frac{\partial W_{x}}{\partial x}+\frac{\partial V}{\partial x} \frac{\partial W_{x}}{\partial p}\right)+2 \ell \frac{\partial\left(p W_{x}\right)}{\partial p}+D_{x} \frac{\partial^{2} W_{x}}{\partial p^{2}}\right\} \\
& +\frac{\partial W_{\gamma}}{\partial W_{\sigma}} \frac{d}{d t} \int d t\left\{\left(-\frac{p}{m} \frac{\partial W_{\sigma}}{\partial \sigma}+\frac{\partial V}{\partial \sigma} \frac{\partial W_{\sigma}}{\partial p}\right)+2 \mu \frac{\partial\left(p W_{\sigma}\right)}{\partial p}+D_{\sigma} \frac{\partial^{2} W_{\sigma}}{\partial p^{2}}\right\}
\end{aligned}
$$

where $V$ is potential energy. The first item in the bracket of equation ( 290 ) is the classic Poisson bracket under the full-view gauge :

$$
\left[H, W_{\gamma}\right]_{\text {Poisson }}=\frac{\partial H}{\partial \gamma} \frac{\partial W_{\gamma}}{p}-\frac{\partial H}{\partial p} \frac{\partial W_{\gamma}}{\partial \gamma},
$$

it is because of $\frac{\partial H}{\partial \gamma}=\frac{\partial V}{\partial \gamma}$ and $\frac{\partial H}{\partial p}=\dot{\gamma}=\frac{p}{m}$. For the general joint-varying system, in the right of equation ( 289 ) there is also the quantum correction with the order of $O(\hbar)$, the 2nd item is damping, the 3 rd item is the diffusion of $W_{\gamma}$ in momentum space, with the diffusion coefficient as $D_{\gamma}$.

\section{Quantum Arche-pulsaton and Its Operator Distrib-Function}

Compared with the energy spectrum, the wave function of the eigenstate contains abundant dynamic information. It can be expected that the gravitation of black holes near the Planck energy scales will reveal a more detailed representation of complexity in quantum mechanics. In order to find out the different characteristics of steady-state wave functions of integrable and non-integrable systems under the semi-classical limit, the coarse-grained average of Wigner distribution can be introduced:

$$
\bar{W}(q, p)=\frac{1}{\Delta^{N}} \int_{q_{1}-\Delta / 2}^{q_{1}+\Delta / 2} d q_{1}^{\prime} \cdots \int_{q_{N}-\Delta / 2}^{q_{N}+\Delta / 2} d q_{N}^{\prime} W\left(q^{\prime}, p^{\prime}\right) .
$$


Under the condition of keeping $\hbar / \Delta \rightarrow 0$, the oscillation of the studied function in the $\hbar$ scale can be averaged out by making $\Delta \rightarrow 0$. M. Berry proved that for integrable system ${ }^{[144]}$, the coarse-grained Wigner distribution in the steady state with action $I_{\psi}$ ( given by WBK quantization condition ${ }^{[145]}$ ) under semi-classical limit is

$$
\overline{W(q, p)}=\delta\left(I(q, p)-I_{\psi}\right) /(2 \pi)^{N}
$$

i.e., $\overline{W(q, p)}$ is a uniform distribution concentrated on the $N$-dimensional torus $I(q, p)=I_{\psi}$.

For the characteristics of irregular state wave functions, it is usually assumed that under semi-classical approximation the coarse-grained Wigner distribution of ergodic nonequilibrium systems can be written as ${ }^{[146]}$

$$
\overline{W(q, p)}=\delta(H(q, p)-E) / \iint d q d p \delta(H(q, p)-E) .
$$

Another quantity showing the morphological characteristics of the irregular state wave function $C$ $(x, q)$ is an autocorrelation function.

After integrating the radial coordinates $|p|$ we get

$$
C(x, q)=\frac{1}{\int d \Omega} \int d \Omega\left\{i \Omega \cdot x[2 m(E-V(q))]^{1 / 2} / \hbar\right\}, \Omega=p /|p| .
$$

Considering Berry's hypothesis of micro-canonical ensemble, we can regard the irregular state wave function $\psi(q)$ under the semi-classical limit as a Gauss-type random function in $q$ space. According to the calculation of M. Shapiro et al. ${ }^{[146]}$, we can get the average autocorrelation function of the billiard model of the stadium: $F(\delta)=\int d q \psi(q+\delta) \psi^{*}(q)$.

For billiards systems, we have $N=2$ and $V(q)=0$, and give $F(\delta)=J_{0}(|\delta| \sqrt{2 m E / \hbar})$. This formula is in good agreement with Shapiro's numerical results for high energy states. The only difference is that when the value of $|\delta|$ is larger than de Broglie wavelength $\hbar / \sqrt{2 m E}$, the numerical results show that $F(\delta)$ has a slightly stronger oscillation than Bessel function of order 0 . However, the nodal pattern, boundary behavior and correlation functions of the wave functions discussed here in configuration space are all characteristics of a single quantum state.

The numerical results of wave functions of some quantum complex behaviors by computer show that ${ }^{[145][146]}$ the actual image of irregular state wave functions is much more complex than that provided by Berry. According to E. J. Heller's calculation of some high-energy wave functions of billiards in sports field ${ }^{[146]}$, about half of the wave functions have obvious structures in configuration space. Surprisingly, the patterns formed by the bulges of these wave functions correspond to the classical trajectories of some unstable periodic orbits of the system. The ratio of the height of the bulge (scar) to the average height depends mainly on the ratio $\omega / \lambda$, which is independent of the size of $\hbar$ ( here $\omega$ is the angular frequency of the periodic orbit and $\lambda$ is the maximum Lyapunov index of the orbit). If $\omega / \lambda$ is larger, the scars caused by periodic orbits become more prominent. Since the width of the scar is 
approximately equal to a de Broglie wavelength, with the increase (or $\hbar$ decrease) of the eigenstate energy, the scar will gradually become thinner and eventually disappear in a uniform background with random fluctuations.

Now, we give the Hamiltonian of arche-pulsaton subsystem $H_{S}=\omega_{c} a^{\dagger} a$, where $a^{\dagger}$ and $a$ are the generators and extinction operators of arche-pulsaton respectively, satisfying the commutative relationship: $\left[a, a^{\dagger}\right]=1$.

$$
\begin{gathered}
\text { Let } Q_{1}=a^{\dagger}, Q_{2}=a, \quad B_{1}=\sum_{i} K_{i} b_{i}, \quad B_{2}=\sum_{i} K_{i}^{*} b_{i}^{\dagger} \text {, then } \\
\omega_{1}=\omega_{c}, \quad \omega_{2}=-\omega_{c}, \\
w_{12}^{+}=\int_{0}^{\infty} d \tau e^{i \omega_{c} \tau} \sum_{i}\left|K_{i}\right|^{2} e^{-i \omega_{i} \tau} \operatorname{Tr}_{B} b_{i} b_{i}^{\dagger} \rho_{B 0}=\left(\bar{n}_{c}+1\right)(K+i \Delta),
\end{gathered}
$$

here $\rho_{B 0}$ is the equilibrium state density matrix of heat reservoir,

$$
\bar{n}_{c}=\operatorname{Tr}_{B} b_{c^{\dagger}} b_{c} \rho_{B 0}=\left(e^{\beta \omega_{c}}-1\right)^{-1}, \quad K+i \Delta=\sum_{i}\left|k_{i}\right|^{2} \int_{0}^{\infty} d \tau e^{i\left(\omega_{c}-\omega_{i}\right) \tau}
$$

In addition, there are $w_{21}^{+}=\bar{n}_{c}(K-i \Delta), w_{12}^{-}=\left(\bar{n}_{c}+1\right)(K-i \Delta), w_{21}^{-}=\bar{n}_{c}(K+i \Delta)$, the equation of motion of damped pulsaton is obtained:

$$
\begin{aligned}
\frac{\partial}{\partial t} \rho_{S}(t) & =-i\left(\omega_{c}+\Delta\right)\left[a^{\dagger} a, \rho_{S}\right] \\
& +K\left\{\left[a, \rho_{S} a^{\dagger}\right]+\left[a \rho_{S}, a^{\dagger}\right]\right\}+2 \bar{n}_{c} K\left[a,\left[\rho_{S}, a^{\dagger}\right]\right]
\end{aligned}
$$

In ( 294 ), we preserve the modification ( $\Delta$ ) of the arche-pulsaton frequency by the quantum cosmic heat reservoir. The steady-state solution of the equation is an equilibrium distribution:

$$
\rho_{S 0}=e^{-\beta a^{\downarrow} a \omega_{c}} / \operatorname{Tr} e^{-\beta \omega_{c} a^{\downarrow} a}{ }^{\dagger} a^{\dagger} .
$$

The physical meaning of the constants in ( 294 ) can be seen directly from the motion equation of the average value of $a^{\dagger}$ and $a^{\dagger} a$ :

$$
\begin{aligned}
& \frac{d}{d t}\left\langle a^{\dagger}\right\rangle=\frac{d}{d t} \operatorname{Tr} \rho_{S}(t) a^{\dagger}=i\left(\omega_{c}+\Delta\right)\left\langle a^{\dagger}\right\rangle-K\left\langle a^{\dagger}\right\rangle \\
& \left.\frac{d}{d t}\left\langle a^{\dagger} a\right\rangle=-2 K\left\langle a^{\dagger} a\right\rangle-\bar{n}_{c}\right)
\end{aligned}
$$

Therefore, $K$ is the average-amplitude attenuation rate of the arche-pulsaton and $\bar{n}_{c} \omega_{c}$ is the energy of the equilibrium average pulsaton.

If the decay time of $\operatorname{Tr}_{B} \rho_{B 0} B(t) B^{\dagger}$ is $\tau_{B}$, then the condition of Markov approximation is $\tau_{B}<<$ $K^{-1}$. Common ordering is adopted and operator distribution function is introduced:

$$
P\left(\alpha, \alpha^{*}, t\right)=\operatorname{Tr} \delta\left(a^{+}-\alpha^{*}\right) \delta(a-\alpha) \rho_{S}(t)
$$

then ( 294 ) is written as

$$
\frac{\partial}{\partial t} P\left(\alpha, \alpha^{*}, t\right)=\operatorname{Tr} \rho_{S}(t)\left\{i\left[H_{S}^{\prime}, e_{N}\right]+K a^{\dagger}\left[e_{N}, a\right]\right.
$$




$$
\left.-K\left[e_{N}, u^{\dagger}\right]+2 K \bar{n}\left[a^{\dagger},\left[e_{N}, a\right]\right]\right\} \delta\left(\alpha^{*}\right) \delta(\alpha)
$$

here $H_{S}^{\prime}=(\omega+\Delta) a^{\dagger} a, e_{N}=e^{-a^{\downarrow} \frac{\partial}{\partial \alpha^{*}}} e^{-\alpha \frac{\partial}{\partial \alpha}}$. Using

$$
\left[a, e^{-a^{\downarrow} \frac{\partial}{\partial \alpha^{*}}}\right]=\frac{\partial}{\partial a^{\downarrow}} e^{-a^{\downarrow} \frac{\partial}{\partial \alpha^{*}}}=-\frac{\partial}{\partial \alpha^{*}} e^{-a^{\downarrow} \frac{\partial}{\partial \alpha^{*}}}, \quad\left[a^{\dagger}, e^{-a \frac{\partial}{\partial \alpha}}\right]=\frac{\partial}{\partial \alpha} e^{-a \frac{\partial}{\partial \alpha}},
$$

we obtain

$$
\begin{gathered}
i\left[H_{S}^{\prime}, e_{N}\right]=i(\omega+\Delta)\left\{a^{\dagger}\left[a, e^{-a^{\downarrow} \frac{\partial}{\partial \alpha^{*}}}\right] e^{-a \frac{\partial}{\partial \alpha}}+e^{-a^{\downarrow} \frac{\partial}{\partial \alpha^{*}}}\left[a^{\dagger}, e^{-a \frac{\partial}{\partial \alpha}}\right] a\right\} \\
=i(\omega+\Delta)\left(e_{N} a \frac{\partial}{\partial \alpha}-a^{\dagger} \frac{\partial}{\partial \alpha^{*}} e_{N}\right) \\
\left.K a^{\dagger}\left[e_{N} a\right]-K\left[e_{N}, a^{\dagger}\right] a=K\left(a^{\dagger} \frac{\partial}{\partial \alpha^{*}} e_{N}+e_{N} a \frac{\partial}{\partial \alpha}\right) a^{\dagger} \frac{\partial}{\partial \alpha^{*}} e_{N}\right) \\
2 K \bar{n}\left[a^{\dagger},\left[e_{N}, a\right]\right]=2 K \bar{n} \frac{\partial^{2}}{\partial \alpha \partial \alpha^{*}} e_{N}
\end{gathered}
$$

After putting them into ( 296 ), we will get

$$
\frac{\partial}{\partial t} P\left(\alpha, \alpha^{*}, t\right)=i(\omega+\Delta)\left(\frac{\partial}{\partial \alpha} \alpha-\frac{\partial}{\partial \alpha^{*}} \alpha^{*}\right) P+K\left(\frac{\partial}{\partial \alpha} \alpha+\frac{\partial}{\partial \alpha^{*}} \alpha^{*}\right) P+2 K \bar{n} \frac{\partial^{2}}{\partial \alpha \partial \alpha^{*}} P
$$

This is a typical Fokker-Planck equation with complex variables. The last one describes the diffusion of $P$ on the $\alpha$ complex plane.

Before solving ( 297 ), the high frequency motion part is eliminated, which is similar to the interaction representation. Setting $\alpha=\beta e^{-i(\omega+\Delta) t}, \alpha^{*}=\beta^{*} e^{i(\omega+\Delta) t}$, then there are

$$
\frac{\partial}{\partial \alpha} \alpha=\frac{\partial}{\partial \beta} \beta, \quad \frac{\partial}{\partial \alpha^{*}} \alpha^{*}=\frac{\partial}{\partial \beta^{*}} \beta^{*}, \quad \frac{\partial^{2}}{\partial \alpha \partial \alpha^{*}}=\frac{\partial^{2}}{\partial \beta \partial \beta^{*}} .
$$

Let $P\left(\alpha, \alpha^{*}, t\right)=q\left(\beta, \beta^{*}, t\right)$, then the damped pulsaton equation can be obtained:

$$
\frac{\partial}{\partial t} q\left(\beta, \beta^{*}, t\right)=K\left(\frac{\partial}{\partial \beta} \beta-\frac{\partial}{\partial \beta^{*}} \beta^{*}\right) q+2 K \bar{n} \frac{\partial^{2}}{\partial \beta \partial \beta^{*}} q=F q
$$

The time behavior of ( 298 ) can be obtained by solving the eigenvalue equation:

$$
F q_{\lambda}=-\lambda q_{\lambda}
$$

Setting the real variables $u$ and $v: \beta=\sqrt{\bar{n}}(u+i v)$, then (299) can be written as

$$
\left\{K\left(u \frac{\partial}{\partial u}+v \frac{\partial}{\partial v}+2\right)+\frac{K}{2}\left(\frac{\partial^{2}}{\partial u^{2}}+\frac{\partial^{2}}{\partial v^{2}}\right)\right\} q(u, v)=\lambda q(u, v)
$$


Taking $q(u, v)=e^{-\left(\frac{u^{2}}{2}+\frac{v^{2}}{2}\right)} Q(u, v)$, we obtain

$$
\frac{\partial^{2}}{\partial u^{2}} Q+\frac{\partial^{2}}{\partial v^{2}} Q+\left(\varepsilon-u^{2}-v^{2}\right) Q=0
$$

here $\varepsilon=\frac{2 \lambda}{K}+2$. the side condition is $u \rightarrow \pm \infty$ or $v \rightarrow \pm \infty, Q \rightarrow 0$. ( 301 ) is the basic motion equation of two-dimensional isotropic harmonic pulsaton, and its eigenvalues

$$
\varepsilon_{n_{1}, n_{2}}=\left(2 n_{1}+1\right)+\left(2 n_{2}+1\right), n_{1}, n_{2}=0,1,2, \cdots,
$$

so that

$$
\lambda_{n_{1}, n_{2}}=K\left(n_{1}+n_{2}\right)
$$

Give the eigenfunction

$$
Q_{n_{1}, n_{2}}=N_{n_{1}, n_{2}} e^{-\frac{1}{2}\left(u^{2}+v^{2}\right)} H_{n_{1}}(u) H_{n_{2}}(v)
$$

here $N_{n_{1}, n_{2}}=\left(\frac{1}{\sqrt{\pi} 2^{n_{1}} n_{1} !} \frac{1}{\sqrt{\pi} 2^{n_{2}} n_{2} !}\right)^{1 / 2}, H_{n}(x)$ is a Hermitian polynomial.

The initial operator distribution function is expanded in terms of ( 303 ). According to ( 302 ), only the part of $n_{1}=n_{2}=0$, i.e. the stationary solution $P_{00}=\frac{1}{\pi \bar{n}} e^{-\frac{1}{\bar{n}}|\beta|^{2}}$, is retained when the time is sufficiently long. This is the Gauss distribution of the thermal fluctuation. If $t=0$ is a coherent state, the transition from coherent state to nonequilibrium state is obtained by retaining the lower order expansion term at time $t \sim K^{-1}$.

\section{Dynamical Base of Quantum Ultra-Synergy Mode}

Now, according to the ultra-synergistic paradigm formed by spin network space, cluster-inflating configuration ( distribution configuration of quantum Physic-Sphere) and interaction situation variables, we discuss the foundation of quantum ultra-synergy mode dynamics between particles and the universe.

In the early universe, quantum gravity is bound to be greatly affected and undergo drastic evolution, in the big rip-rebound clusters of quantum chaotic-reticulate distribution emerged between the outwards-push of the universe as a whole (related to pseudo-vacuum energy, Higgs field, dark energy, etc.) and the inwards-pull of the universe in local scale (related to dark matter, quantum gravity and initial gravity separated from high-dimensional supergravity, and the initial strong force separated from the grand unified force subsequently). Here, each quantum gravity can be regarded as a minimal "open system" that evolves from an initial chaotic state to a nonequilibrium state and undergoes instantaneous relaxation process, i.e. a micro evolution system coupled with the early universe (which can be called a "heat reservoir"). Since the early quantum universe as a heat reservoir is sufficiently 
large relative to quantum gravity (with sufficient degrees of freedom), and its internal relaxation process is also very fast, it can be considered that the early quantum universe is basically in equilibrium. Because the quantum gravity we are concerned about is extremely small, it is necessary to describe its motion behavior with quantum theory.

We discuss the motion behavior of the mechanical quantity of the quantum gravi-system $S$ in the early quantum universe ( large heat reservoir ) $B$, which corresponds to the Heisenberg image. The Hamiltonian of total system $B \oplus S$

$$
H_{T O L}=H_{B}+H_{S}+H_{S B}=H_{0}+H_{S B}
$$

here $H_{B}$ is the Hamiltionian of $B$ itself, $H_{S}$ is the Hamiltionian of $S$ itself, Accordingly, the Liouville operator can be written as

$$
L_{T O L}=L_{B}+L_{S}+L_{S B}=L_{0}+L_{S B}
$$

All the mechanical quantities of the total system $B \oplus S$ constitute the $C^{*}$ algebra $u^{T O L}$.

If the temperature of the quantum universe as a heat reservoir is $\beta^{-1}$, the inner product can be introduced into $u^{T O L}$ :

$$
\left(K_{1}, K_{2}\right)=\operatorname{Tr} K_{1}^{+} K_{2} \rho_{T O L, 0}, \quad K_{1}, K_{2} \in u^{T O L}
$$

here $\rho_{T O L, 0}=e^{-\beta H_{T O L}} / \operatorname{Tr} e^{-\beta H_{T O L}}$. By formula ( 304 ), we introduce the distance

$$
\left\|K_{1}-K_{2}\right\|=\sqrt{\left(K_{1}-K_{2}, K_{1}-K_{2}\right)}
$$

We can make $u^{T O L}$ complete and get the Hilbert space $\mathscr{H}_{\beta}{ }^{T O L}$. The total Liouville operator $L$ is Hermitian in $\mathscr{H}_{\beta}{ }^{T O L}$. What we really care about is the paradigm vectors of the quantum gravi-system $S$, which constitute a subspace $\mathscr{H}_{\beta}{ }^{S}$ of $\mathscr{H}_{\beta}{ }^{T O L}$. If the vector $\underline{e}=\left\{e_{i}\right\}_{i}$ is a set of bases in $\mathscr{H}_{\beta}{ }^{T O L}$, then the motion of the $S$ system is completely given by the temporal behavior of $\underline{e}(t)$.

Now, we introduce the projection operator:

$$
P A=(A, \underline{e}) \cdot(\underline{e} \cdot \underline{e})^{-1} \cdot \underline{e}, \quad A \in \mathscr{H}_{\beta}^{T O L}
$$

here $(\underline{e} \cdot \underline{e})^{-1}$ is the inverse matrix of dyadic ( $\left.\underline{e} \cdot \underline{e}\right)$. After separating $P$ and $Q=1-P$ and solving $Q$ motion formally, it is not difficult to obtain the generalized Langevin equation satisfied by $\underline{e}(t)$ :

$$
\frac{d}{d t} \underline{e}(t)=i Q \cdot \underline{e}(t)-\int_{0}^{t} d \tau K(\tau) \cdot \underline{e}(t-\tau)+\underline{F}(t)
$$

here

$$
i Q=(\underline{\dot{e}} \cdot \underline{e}) \cdot(\underline{e} \cdot \underline{e})^{-1}, \quad \underline{F}(t)=e^{i Q L Q t} Q \underline{\dot{e}}(0), \quad K(\tau)=-i(\underline{F}(\tau) \underline{F}(0)) \cdot(\underline{e} \cdot \underline{e})^{-1}
$$

and $(\underline{F}(t) \underline{e}(0))=0, t>0,(305)$ is equivalent to the Liouville equation of the total system $B \oplus S$. However, because the quantum universe as a heat reservoir is very large relative to the quantum gravisystem and has strong internal relaxation, its correlation function $K(\tau)$ decays rapidly with $\tau$ (because $Q \dot{e}$ is only a mechanical quantity of heat reservoir), or in other words, the transformation of the $\underline{e}(t)$ 
of the $S$ system within the decay time $\tau_{W}\left(t>>\tau_{W}\right)$ of $K(\tau)$ is very small and Markov approximation can be used:

$$
\int_{0}^{t} K(t-\tau) \cdot \underline{e}(\tau) d \tau \sim \theta \cdot \underline{e}(t)
$$

here $\theta=\int_{0}^{\infty} K(\tau) d \tau$, thus Langevin equation is obtained:

$$
\frac{d}{d t} \underline{e}(t)=(i Q-\theta) \cdot \underline{e}(t)+\underline{F}(t)
$$

$\underline{F}(t)$ is the fluctuating force and $\theta$ is the generalized friction coefficient (dissipation coefficient). Taking the limit $\tau_{W} \rightarrow 0$, the correlation function of $\underline{F}(t)$ has white spectrum, and $K(\tau)$ can be rewritten as follows

$$
K(\tau)=-i(\underline{F}(t) \underline{F}(0)) \cdot(\underline{e} \cdot \underline{e})^{-1}=\theta \delta(t)
$$

This formula directly relates the friction coefficient $\theta$ in Markov process ( 308 ) to the fluctuation force $\underline{F}$. In other words, it links the dissipation coefficient of our early quantum universe $S$ with the motion fluctuation of the Multi-verse world system as a heat reservoir, and introduces statistical factors (Markov approximation) in the derivation process.

In the quantum Physic-Spheres studied in this series, boiling at ultra-high temperature is everywhere, and many vacuum bubbles are scattered. At this moment, the pseudo-vacuum decays into the real vacuum, the grand unified symmetry is broken, and there is a synergistic derivation mechanism of the initial cosmic interaction system. So it is possible to reveal the Higgs effect in a more natural way.

In the expansion of the universe with the big impetus, the whole energy density decreases as the volume increases, while the density of ordinary "cold" materials is inversely proportional to the volume, the energy density is inversely proportional to the cubic of volume, and the radiation energy is inversely proportional to the cubic of volume.

In the rip-rebound-inflation process of the universe with the big impetus, the energy density of the inflation field will fluctuate with the emergence of big rip-rebound clusters with quantum chaoticreticulate distribution in the interaction of the quantum universe, while the inhomogeneity, anisotropy, spatial curvature and the number density of various singular particles will be reduced, and the number density of photons will also be reduced. After the rip-rebound-inflation with the big impetus, it will be reduced to a negligible degree. Thus, a nearly vacuum, flat, symmetrical universe will be created, and then it will go into reheating.

In fact, describing the open quantum gravi-system with the generalized Langevin equation ( 308 ) is to divide the forces acting on the quantum gravity by the quantum universe as a heat reservoir into two parts: one is the average force, i.e. the generalized friction force $-\theta \underline{e}(t)$, and the other is the random force $\underline{F}$ (the average is zero). When the distinction (actually the projection operator $P$ ) between the quantum gravity as an open system and the quantum universe as a heat reservoir has been established, this division of the forces of the quantum universe on the quantum gravity is unique. However, for a specific problem, how to distinguish the quantum gravity at a later time $(t>0)$ has changed. Generally speaking, the choice of projection operator $P$ is different, and the results are different. In the above derivation, the choice of different $P$ is expressed as the definition of different 
inner product. We take as ( 304 )

$$
\left(K_{1}, K_{2}\right)=\operatorname{Tr} K_{1}^{\dagger} K_{2} \rho_{T O L, 0}
$$

Even though $K_{1}$ and $K_{2}$ are only mechanical quantities of the quantum universe $S,\left(K_{1}, K_{2}\right)$ is related to the multi-verse world system $W$ by $H_{U W}$ in $H_{T O L}$. Specific calculations ( 304 ) will be difficult. If defined

$$
\left(K_{1}, K_{2}\right)=\operatorname{Tr} K_{1}^{\dagger} K_{2} \rho_{0}
$$

here $\rho_{, 0}=e^{-\beta H_{0}} /$ Tre $e^{-\beta H_{0}}$, then, the concrete calculation will be much simpler; however, the Liouville operator $L$ is no longer Hermitian operator under the definition of this inner product, so it should be taken in the derivation ( 305 )

$$
(L Q \underline{F}(\tau), \underline{e})=\left(\underline{F}(\tau), Q L^{\dagger} \underline{e}\right)=i\left(\underline{F}(\tau), \underline{F}^{\dagger}\right)
$$

here $\underline{F}^{\dagger}=-i Q L^{\dagger} \underline{e}$. When the Markov limit is taken, the obtained correlation function is

$$
K(\tau)=-i\left(\underline{F}(\tau) \underline{F}(0)^{\dagger}\right) \cdot(\underline{e} \cdot \underline{e})^{-1}=\theta \delta(\tau)
$$

For the case of real fluctuation force (in many classical cases, $\underline{F}$ is a real function), ( 311 ) is the same as ( 309 ), and the fluctuation dissipation relationship remains unchanged. However, if $\underline{F}(t)$ is complex, then ( 311 ) is different from ( 309 ). As long as $\underline{e}$ is Hermitian, we can think that ( 311 ) is to satisfy the fluctuation dissipation relationship. So, in most cases, the inner product is defined by ( 310 ). If $\underline{e}$ is taken as a set of orthogonal normalized complete bases, Langevin equation can be obtained.

After the Big Bang, there will be some remnants, such as microwave background radiation, reflecting small fluctuations of temperature long after the Big Bang (380000 years); for example, there will be adiabaticity, Gaussian density distribution, scaling invariance of density fluctuations, where there is polarization distribution (E mode and B mode), which reveals the statistical information of the universe.

The inflation cosmic model tells us that only through exponential inflation can the universe appear flat, homogeneous and isotropic on the largest scale ${ }^{[28]-[30]}$. It is generally believed that only when the universe expands at a rate greater than $e^{60}\left(\approx 10^{26}\right)$ in the stage of inflation can it meet this requirement. The observed data from the cosmic microwave background have verified that the universe is flat and the error value is less than $10 \%$, which makes the problem of cosmic flatness more significant.

The rip-rebound-inflation with the big impetus is a supercooled expansion stage, during which the temperature of the universe decreases by 100,000 times (it is generally believed that in the earliest model, the temperature dropped from $10{ }^{27} \mathrm{~K}$ to $10^{22} \mathrm{~K}$ ), and then the temperature remained at a relatively low temperature. When inflation is over, the temperature returns to its pre-inflation level ( from $10^{22} \mathrm{~K}$ to $10^{27} \mathrm{~K}$ ), this process is known as "reheating" or "heating". This is because the huge potential energy in the rip-rebound-inflation field decays into various particles, filling the universe with standard model particles, including electromagnetic radiation, thus launching the radiation dominant period. 
The rip-rebound-inflation can also reduce the numerical density of large-mass singular particles, such as magnetic monopoles predicted by many derivative theories of the standard model of particle physics. If the universe had enough temperature to form these particles only before the rip-reboundinflation period, the rip-rebound-inflation would reduce their density to a very low level, so that they do not exist in today's visible universe. Taken together, these effects can be called the "hairless theorem" of inflation, similar to the black hole hairless theorem.

\section{Master Equation of Ultra-Synergy Mode Dynamics}

The generalized Langevin equation can be regarded as the mode dynamical equation of the open quantum gravi-system $S$ in Heisenberg's image, and the density matrix motion equation of the quantum gravi-system $S$ can also be discussed from Schrodinger's image (Liouville equation).

The density matrix $\rho$ TоL of ultra-synergistic system $B \oplus S$ satisfies the liouville Equation:

$$
\frac{d}{d t} \rho_{T O L}=-i\left(L_{0}+L_{B S}\right) \rho_{T O L}
$$

here $L_{0}=L_{B}+L_{S}$. Of course, we only focus on the mean value of the mode vector of the quantum gravi-system $S$ :

$$
<e>=\operatorname{Tr} e \rho_{T O L}=\operatorname{Tr}_{S} e\left(\operatorname{Tr}_{B} \rho_{T O L}\right)=\operatorname{Tr}_{S} e \rho_{S}
$$

Therefore, we hope to get the equation of motion satisfied by $\rho_{s}$. Setting projection operator $P$ :

$$
P \rho_{T O L}=\rho_{B_{0}} \operatorname{Tr}_{B} \rho_{T O L}
$$

$\rho_{B 0}$ is a traceable operator of $B$ system: $\operatorname{Tr}_{B} \rho_{B_{0}}=1$, its selection depends on the specific problem.

Let $Q=1-P$, so we get

$$
\frac{d}{d t} \rho_{S}(t)=-i L_{u, S} \cdot \rho_{S}(t)-\int_{0}^{t} d \tau K(t-\tau) \cdot \rho_{S}(\tau)+F(t)
$$

here it uses $P L_{B}=0$ and $P L_{S}=L_{S} P$, and there is

$$
\begin{gathered}
L_{u, S}=\operatorname{Tr}_{B} L \rho_{B_{0}}=L_{S}+\operatorname{Tr}_{B} L_{B S} \rho_{B_{0}} \\
K(\tau)=\rho_{B 0}^{-1} P L Q e^{-i Q L Q \tau} Q L P=\operatorname{Tr}_{B} L_{B S} e^{-i Q L Q \tau} Q\left(L_{B}+L_{B S}\right) \rho_{B 0}
\end{gathered}
$$

If $\rho_{B 0}$ satisfies $L_{B} \rho_{B_{0}}=0$, then

$$
K(\tau)=\operatorname{Tr}_{B} L_{B S} e^{-i Q L Q \tau} L_{B S} \rho_{B 0}
$$

Equation ( 315 ) holds for any chosen $\rho_{B 0}$ ( arbitrary $P$ ), and generally does not bring more convenience for solution.

Considering that the quantum cosmic system as a heat reservoir is relatively large, the internal relaxation is very strong, and the effect of $S$ motion on $B$ is very small, we can take

$$
\rho_{B, 0}=e^{-\beta H_{B}} / \operatorname{Tr} e^{-\beta H_{B}}
$$


then take the regular distribution of $B$. In this way, $Q L Q$ only contains the non-equilibrium motion of $B$ system, and $K(\tau)$ should decay rapidly. However, $\rho_{S}(t)$ does not necessarily slow down. The fast change of $\rho_{S}(t)$ is mainly caused by $L_{S}$, so it can be considered that

$$
\rho_{S}^{\prime}(t)=e^{i L_{S} t} \rho_{S}(t)
$$

is slow change. Therefore, if only the lowest order of $L_{B S}$ is considered and $F(t)$ is omitted, we can rewrite ( 315 ) to

$$
\frac{d}{d t} \rho_{S}(t)=-i L_{e, S} \cdot \rho_{S}(t)-\int_{0}^{t} d \tau \operatorname{Tr}\left(L_{B S} e^{-i L_{0} \tau} L_{B S} \rho_{B 0}\right) e^{-i L_{S}(t-\tau)} \cdot \rho_{S}^{\prime}(t-\tau)
$$

If the change of $K(\tau)$ is much faster than that of $\rho^{\prime}{ }_{U}(t)$, the Markov approximation can be introduced :

$$
\int_{0}^{t} d \tau K(\tau) e^{-i L_{S} t} \cdot \rho_{S}^{\prime}(t-\tau) \approx \int_{0}^{\infty} d \tau K(\tau) \rho_{S}(t)=\theta \rho_{S}(t)
$$

We get Master equation :

$$
\frac{d}{d t} \rho_{S}(t)=-i L_{e, S} \cdot \rho_{S}(t)-\theta \cdot \rho_{S}(t)
$$

The coupling of S and B systems can be written in general :

$$
H_{B S}=\sum_{j} Q_{j} B_{j}
$$

here $Q_{j}$ is the operator of the quantum gravi-system $S$ and $B_{j}$ is the operator of the quantum cosmic heat reservoir. Taking $Q_{j}$ as the eigenvector of $L_{S}$, we have

$$
e^{-i L_{s} t} Q_{j}=e^{-i \omega_{j} t} Q_{j}
$$

So only the lowest order of $L_{B S}$ is obtained, then

$$
\begin{aligned}
K(\tau) \rho_{S}(t) & =\operatorname{Tr}_{B}\left(L_{B S} e^{-i L_{0} \tau} L_{B S} \rho_{B 0}\right) \rho_{S}(t) \\
& =\sum_{k, j} e^{-i \omega_{j} \tau} \operatorname{Tr}_{B}\left\{Q_{k} B_{k},\left[e^{-i L_{B} \tau} Q_{j} B_{j}, \rho_{B 0} \rho_{S}(t)\right]\right\} \\
& =\sum_{k, j} e^{-i \omega_{j} \tau}\left\{\left[Q_{k}, Q_{j} \rho_{S}(t)\right] \operatorname{Tr}_{B} B_{k}(\tau) B_{j} \rho_{B 0}-\left[Q_{k}, \rho_{S}(t) Q_{j}\right] \operatorname{Tr}_{B} B_{j} B_{k}(\tau) \rho_{B 0}\right\}
\end{aligned}
$$

taking

$$
w_{k j}^{+}=\int_{0}^{\infty} d \tau e^{-i \omega_{j} \tau} \operatorname{Tr}_{B} B_{k}(\tau) B_{j} \rho_{B 0}, \quad w_{k j}^{-}=\int_{0}^{\infty} d \tau e^{-i \omega_{j} \tau} \operatorname{Tr}_{B} B_{j} B_{k}(\tau) \rho_{B 0}
$$

then ( 318 ) Can be further written

$$
\frac{d}{d t} \rho_{S}(t)=-i L_{e, S} \cdot \rho_{S}(t)-\sum_{k, j}\left\{\left[Q_{k}, Q_{j} \rho_{S}(t)\right] w_{k j}^{+}-\left[Q_{k}, \rho_{S}(t) Q_{j}\right] w_{j k}^{-}\right\}
$$

This is the generalized Master equation of the most general open system. Obviously, ( 322 ) will keep the trace of $\rho_{S}(t)$ unchanged: $\frac{d}{d t} \operatorname{Tr}_{S} \rho_{S}(t)=0$.

Usually, the imaginary part of $w^{ \pm}$can be neglected, and the main contribution to sum comes from the term $w_{k}+w_{j}=0$.

We can also directly transit from ( 322 ) to Heisenberg image and obtain the mode dynamical 
equation of quantum gravi-system. From

$$
\begin{aligned}
\frac{d}{d t} \operatorname{Tr}_{S} e_{i} \rho_{S}(t) & =\operatorname{Tr}_{S} e_{i} \frac{d}{d t} \rho_{S}(t) \\
& =\operatorname{Tr}_{S} e_{i}\left\{-i L_{e, S} \rho_{S}(t)-\theta \rho_{S}(t)\right\}=\operatorname{Tr}_{S}\left\{-i L_{e r} e_{i}-\theta^{\dagger} e_{i}\right\} \rho_{S}(t)
\end{aligned}
$$

we obtain

$$
\frac{d}{d t} e_{i}(t)=i L_{e, S} e_{i}(t)-\theta^{\dagger} e_{i}(t)
$$

here

$$
\theta^{\dagger} e_{i}=\sum_{j, k}\left\{w_{k j}^{+}\left[e_{i}, Q_{k}\right] Q_{j}-w_{j k}^{-} Q_{j}\left[e_{i}, Q_{k}\right]\right\}
$$

At the beginning of the creation of the universe $\left(10^{-42} \mathrm{sec} \sim 10^{-36} \mathrm{sec}\right)$, the quantum gravi-system $S$ can be seen as a coupling $S=S_{1} \oplus S_{2}$ of the gravitational system $S_{1}$ and the gauge field force $S_{2}$.

If the heat source ( $Q$ part) relaxes rapidly and is weakly coupled with $S$ system, the following equations are given:

$$
\frac{d}{d t} \rho_{12}(t)=-i \mathscr{L} \rho_{12}(t)=-i \tilde{L}_{12} \rho_{12}(t)-\tilde{\theta}_{12} \rho_{12}(t)
$$

here

$$
\begin{aligned}
& \tilde{L}_{12}=L_{1}+L_{2}+L_{12} \\
& \tilde{\theta}_{12}=\theta_{1}+\theta_{2}+\theta_{12} \\
& \theta_{j}=\int_{0}^{\infty} \operatorname{Tr}_{B} L_{j, B} e^{-i L_{0} \tau} L_{j, B} \rho_{B 0} d \tau \quad j=1,2 \\
& \theta_{12}=\int_{0}^{\infty} \operatorname{Tr}_{B}\left(L_{1, B} e^{-i L_{0} \tau} L_{2, B}+L_{2, B} e^{-i L_{0} \tau} L_{1, B}\right) \rho_{B 0} d \tau
\end{aligned}
$$

Taking projection operator $P_{1}: P_{1} \rho_{12}=\rho_{20} \operatorname{Tr}_{2} \rho_{12}=\rho_{20} \rho_{1}, Q_{1}=1-P_{1}$, and because of $L_{2} \rho_{20}$ $=0$, there is

$$
\frac{\partial}{\partial t} \rho_{1}(t)=-i L_{1} \rho_{1}(t)-\int_{-\infty}^{t} K_{12}(t, \tau) \rho_{1}(\tau) d \tau
$$

here

$$
K_{12}(t, \tau)=\rho_{20}^{-1} P_{1} L_{12} e^{-i \int_{\tau}^{t} d \tau^{\prime} Q \tilde{L}_{12} Q} L_{12} \rho_{20}
$$

$K_{12}$ has time translation invariance:

$$
K_{12}(t-\tau)=\operatorname{Tr}_{2} L_{12} e^{-Q\left[i\left(L_{1}+L_{2}+L_{12}\right)+\theta_{2}\right] Q(t-\tau)} L_{12} \rho_{20}
$$

so there is

$$
\frac{\partial}{\partial t} \rho_{1}(t)=-i L_{1} \rho_{1}(t)-\int_{-\infty}^{t} d \tau K_{12}(\tau) \rho_{1}(t-\tau)
$$

If the relaxation of system $U_{2}$ is much faster than that of system $U_{1}$, then we get 


$$
\frac{\partial}{\partial t} \rho_{1}(t)+i L_{1} \rho_{1}(t)=-\theta \rho_{1}(t)
$$

here

$$
\theta=\int_{0}^{\infty} d \tau K_{12}(\tau)=\operatorname{Tr}_{2} L_{12} \frac{1}{i\left(L_{1}+L_{2}+L_{12}\right)+\theta_{2}} L_{12} \rho_{20}
$$

\section{Calculation of Black Hole HLX-1 as An Example}

Now, taking HLX-1, a medium-sized black hole, as an example, the relevant numerical calculation is given. The black hole HLX-1 has a radius of $295,300 \mathrm{~km}$, the size of four Jupiters side by side, but it has a mass equivalent to 100,000 suns.

Here, let the spin network space of a quantum Physic-Sphere have $A$ sides, namely: spin quantum number $\mu_{1}$, spin angular momentum $\mu_{2}$, space dimension (including integer dimension and fractal dimension, as well as high dimension and low dimension) $\mu_{3}$, acting radius $\mu_{4}$, node number $\mu_{5}$, concentration degree of network node distribution $\mu_{6}$, and so on, Table 1 (Li Zongcheng, 2019) gives the following specific results:

network space 001: $S N[001]=(\{0,2\}, \geq 10$ (extended 4 dimensions), $0.0000000001 \times 295$, $\left.300 \mathrm{~km}, 25.54677 \times 10^{99}, 1.00000000\right)$;

network space 002: $S N[002]=(\{0,2\}, \geq 10$ (extended 4 dimensions), $0.0000000010 \times 295$, $\left.300 \mathrm{~km}, 10^{3} \times 25.54677 \times 10^{99}, 1.5924 \times 10^{-4}\right)$;

network space 003: $S N[003]=\left(\{0,2\},\left(\frac{\hbar}{2} \hat{\sigma}\right)^{2}, \geq 10\right.$ (extended 4 dimensions),

$\left.0.0000000101 \times 295,300 \mathrm{~km}, 10^{6} \times 25.54677 \times 10^{99}, 0.0000000149 \times 10^{-4}\right)$;

network space 004: $S N[004]=\left(\{0,2\},\left(\frac{\hbar}{2} \hat{\sigma}\right)^{2}, \geq 10\right.$ (extended 4 dimensions),

$\left.0.0000001001 \times 295,300 \mathrm{~km}, 10^{9} \times 25.54677 \times 10^{99}, 1.44209 \times 10^{-6}\right)$;

network space 005: $S N[005]=\left(\{0,2\},\left(\frac{\hbar}{2} \hat{\sigma}\right)^{2}, \geq 10\right.$ (extended 4 dimensions),

$\left.0.0000010001 \times 295,300 \mathrm{~km}, 10^{12} \times 25.54677 \times 10^{99}, 0.88846 \times 10^{-7}\right)$;

network space 006: $S N[006]=\left(\{0,2\},\left(\frac{\hbar}{2} \hat{\sigma}\right)^{2}, \geq 10\right.$ (extended 4 dimensions),

$\left.0.0000100001 \times 295,300 \mathrm{~km}, 10^{15} \times 25.54677 \times 10^{99}, 0.67682 \times 10^{-8}\right)$;

network space 007: $S N[007]=\left(\{0,2\},\left(\frac{\hbar}{2 \pi},\left(\frac{\hbar}{2} \hat{\sigma}\right)^{2}\right), \geq 10\right.$ (extended 4 dimensions),

$\left.0.0001000001 \times 295,10^{18} \times 300 \mathrm{~km}, 25.54677 \times 10^{99}, 0.20825 \times 10^{-10}\right)$; 
Table 1 Spin Network Space Centered on HLX-1 Black Hole

\begin{tabular}{|c|c|c|c|c|c|c|}
\hline & $\begin{array}{l}\text { spin quan } \\
\text { number } \\
S 01\end{array}$ & $\begin{array}{l}\text { spin angular } \\
\text { momentum } \\
\quad S 02\end{array}$ & $\begin{array}{c}\text { spatial } \\
\text { dimension } \\
S 03\end{array}$ & $\begin{array}{l}\text { action } \\
\text { radius } \\
S 04\end{array}$ & $\begin{array}{c}\text { network node } \\
\text { number } \\
S 05\end{array}$ & $\begin{array}{c}\text { concentration } \\
\text { ratio } \\
S 06\end{array}$ \\
\hline $\begin{array}{l}\text { spin net space } \\
\text { SN } 01\end{array}$ & $\begin{array}{l}0 \quad \text { Higgs } \\
2 \text { graviton }\end{array}$ & & $\begin{array}{l}\leq 10 \text { exten } \\
4 \text { dimsion }\end{array}$ & $\begin{array}{c}0.0000000001 \times \\
295,300 \mathrm{~km}\end{array}$ & $\begin{array}{c}25.54677 \\
\times 10^{99}\end{array}$ & 1 \\
\hline $\begin{array}{l}\text { spin net space } \\
\text { SN } 02\end{array}$ & $\begin{array}{l}0 \quad \text { Higgs } \\
2 \text { graviton }\end{array}$ & & $\begin{array}{l}\leq 10 \text { exten } \\
4 \text { dimsion }\end{array}$ & $\begin{array}{c}0.0000000010 \times \\
295,300 \mathrm{~km}\end{array}$ & $\begin{array}{c}10^{3} \times 25.54677 \\
\times 10^{99}\end{array}$ & $1.5924 \times 10^{-4}$ \\
\hline $\begin{array}{l}\text { spin net space } \\
\qquad N 03\end{array}$ & $\begin{array}{l}0 \text { Higgs } \\
2 \text { graviton }\end{array}$ & $\left(\frac{\hbar}{2} \hat{\sigma}\right)^{2}$ & $\begin{array}{l}\leq 10 \text { exten } \\
4 \text { dimsion }\end{array}$ & $\begin{array}{c}0.0000000101 \times \\
295,300 \mathrm{~km}\end{array}$ & $\begin{array}{c}10^{6} \times 25.54677 \\
\times 10^{99}\end{array}$ & $\begin{array}{c}0.0000000149 \\
\times 10^{-4}\end{array}$ \\
\hline $\begin{array}{c}\text { spin net space } \\
\text { SN } 04\end{array}$ & $\begin{array}{l}0 \quad \text { Higgs } \\
2 \text { graviton }\end{array}$ & $\left(\frac{\hbar}{2} \hat{\sigma}\right)^{2}$ & $\begin{array}{l}\leq 10 \text { exten } \\
4 \text { dimsion }\end{array}$ & $\begin{array}{c}0.0000001001 \times \\
295,300 \mathrm{~km}\end{array}$ & $\begin{array}{c}10^{9} \times 25.54677 \\
\times 10^{99}\end{array}$ & $\begin{array}{r}1.44209 \\
\times 10^{-6}\end{array}$ \\
\hline $\begin{array}{l}\text { spin net space } \\
\text { SN } 05\end{array}$ & $\begin{array}{l}0 \text { Higgs } \\
2 \text { graviton }\end{array}$ & $\left(\frac{\hbar}{2} \hat{\sigma}\right)^{2}$ & $\begin{array}{l}\leq 10 \text { exten } \\
4 \text { dimsion }\end{array}$ & $\begin{array}{c}0.0000010001 \times \\
295,300 \mathrm{~km}\end{array}$ & $\begin{array}{c}10^{12} \times 25.54677 \\
\times 10^{99}\end{array}$ & $\begin{array}{r}0.88846 \\
\times 10^{-7}\end{array}$ \\
\hline $\begin{array}{l}\text { spin net space } \\
\qquad N 06\end{array}$ & $\begin{array}{l}0 \quad \text { Higgs } \\
2 \text { graviton }\end{array}$ & $\left(\frac{\hbar}{2} \hat{\sigma}\right)^{2}$ & $\begin{array}{l}\leq 10 \text { exten } \\
4 \text { dimsion }\end{array}$ & $\begin{array}{c}0.0000100001 \times \\
295,300 \mathrm{~km}\end{array}$ & $\begin{array}{c}10^{15} \times 25.54677 \\
\times 10^{99}\end{array}$ & $\begin{array}{r}0.67682 \\
\times 10^{-8} \\
\end{array}$ \\
\hline $\begin{array}{l}\text { spin net space } \\
\text { SN } 07\end{array}$ & $\begin{array}{l}0 \quad \text { Higgs } \\
2 \text { graviton }\end{array}$ & $\frac{\hbar}{2 \pi},\left(\frac{\hbar}{2} \hat{\sigma}\right)^{2}$ & $\begin{array}{l}\leq 10 \text { exten } \\
4 \text { dimsion }\end{array}$ & $\begin{array}{c}0.0001000001 \times \\
295,300 \mathrm{~km}\end{array}$ & $\begin{array}{c}10^{18} \times 25.54677 \\
\times 10^{99}\end{array}$ & $\begin{array}{l}0.20825 \\
\times 10^{-10}\end{array}$ \\
\hline $\begin{array}{l}\text { spin net space } \\
\qquad N 08\end{array}$ & $\begin{array}{l}0 \quad \text { Higgs } \\
2 \text { graviton }\end{array}$ & $\frac{\hbar}{2 \pi},\left(\frac{\hbar}{2} \hat{\sigma}\right)^{2}$ & $\begin{array}{l}\leq 10 \text { exten } \\
4 \text { dimsion }\end{array}$ & $\begin{array}{c}0.0010000001 \times \\
295,300 \mathrm{~km}\end{array}$ & $\begin{array}{c}10^{21} \times 25.54677 \\
\times 10^{99}\end{array}$ & $\begin{array}{r}0.12566 \\
\times 10^{-16}\end{array}$ \\
\hline $\begin{array}{l}\text { spin net space } \\
\text { SN } 09\end{array}$ & $\begin{array}{l}0 \quad \text { Higgs } \\
2 \text { graviton }\end{array}$ & $\frac{\hbar}{2 \pi},\left(\frac{\hbar}{2} \hat{\sigma}\right)^{2}$ & $\begin{array}{l}\leq 10 \text { exten } \\
4 \text { dimsion }\end{array}$ & $\begin{array}{c}0.0100000001 \times \\
295,300 \mathrm{~km}\end{array}$ & $\begin{array}{c}10^{24} \times 25.54677 \\
\times 10^{99}\end{array}$ & $\begin{array}{r}1.159707 \\
\times 10^{-37} \\
\end{array}$ \\
\hline $\begin{array}{l}\text { spin net space } \\
\text { SN } 10\end{array}$ & $\begin{array}{l}0 \text { Higgs } \\
2 \text { graviton }\end{array}$ & $\frac{\hbar}{2 \pi},\left(\frac{\hbar}{2} \hat{\sigma}\right)^{2}$ & $\begin{array}{l}\leq 10 \text { exten } \\
4 \text { dimsion }\end{array}$ & $\begin{array}{c}0.1000000001 \times \\
295,300 \mathrm{~km}\end{array}$ & $\begin{array}{c}10^{27} \times 25.54677 \\
\times 10^{99}\end{array}$ & $\begin{array}{r}0.67546 \\
\times 10^{-46}\end{array}$ \\
\hline $\begin{array}{l}\text { spin net space } \\
\qquad N 11\end{array}$ & $\begin{array}{l}0 \quad \text { Higgs } \\
2 \text { graviton }\end{array}$ & $\frac{\hbar}{2 \pi},\left(\frac{\hbar}{2} \hat{\sigma}\right)^{2}$ & $\begin{array}{l}\leq 10 \text { exten } \\
4 \text { dimsion }\end{array}$ & $\begin{array}{c}1.0000000001 \times \\
295,300 \mathrm{~km}\end{array}$ & $\begin{array}{c}10^{30} \times 25.54677 \\
\times 10^{99}\end{array}$ & $\begin{array}{r}1.46779 \\
\times 10^{-61}\end{array}$ \\
\hline
\end{tabular}

network space 008: $S N[008]=\left(\{0,2\},\left(\frac{\hbar}{2 \pi},\left(\frac{\hbar}{2} \hat{\sigma}\right)^{2}\right), \geq 10\right.$ (extended 4 dimensions), $\left.0.0010000001 \times 295,10^{21} \times 300 \mathrm{~km}, 25.54677 \times 10^{99}, 0.12566 \times 10^{-16}\right)$;

network space 009: $S N[009]=\left(\{0,2\},\left(\frac{\hbar}{2 \pi},\left(\frac{\hbar}{2} \hat{\sigma}\right)^{2}\right), \geq 10\right.$ (extended 4 dimensions),

$\left.0.0100000001 \times 295,10^{24} \times 300 \mathrm{~km}, 25.54677 \times 10^{99}, 1.159707 \times 10^{-37}\right)$;

network space 010: $S N[010]=\left(\{0,2\},\left(\frac{\hbar}{2 \pi},\left(\frac{\hbar}{2} \hat{\sigma}\right)^{2}\right), \geq 10\right.$ (extended 4 dimensions), $\left.0.1000000001 \times 295,10^{27} \times 300 \mathrm{~km}, 25.54677 \times 10^{99}, 0.67546 \times 10^{-46}\right)$; 
network space 011: $S N[011]=\left(\{0,2\},\left(\frac{\hbar}{2 \pi},\left(\frac{\hbar}{2} \hat{\sigma}\right)^{2}\right), \geq 10\right.$ (extended 4 dimensions),

$\left.1.000000001 \times 295,10^{30} \times 300 \mathrm{~km}, 25.54677 \times 10^{99}, 1.46779 \times 10^{-61}\right)$.

For the ultra-synergy model, we consider the following components: $w_{1}$. strength, $w_{2}$. force range, $w_{3}$. mass density, $w_{4}$. the inverse ratio to the square of the gravitational distance $\left(1 / S^{2}\right), w_{5}$. spin quantum number, $w_{6}$. concentration, etc. Table 2 (Li Zongcheng, 2019) gives the following specific results:

Table 2 ultra-synergy mode Vectors Centered on HLX-1 Black Hole

\begin{tabular}{|c|c|c|c|c|c|c|}
\hline & $\begin{array}{c}\text { gravitational } \\
\text { strength } \\
\quad w 01\end{array}$ & $\begin{array}{l}\text { interaction } \\
\quad \text { rang } \\
\quad w 02\end{array}$ & $\begin{array}{l}\text { mass } \\
\text { density } \\
w 03\end{array}$ & $\begin{array}{l}\text { inverse } \\
\text { ratio } 1 / S^{2} \\
\quad w 04\end{array}$ & $\begin{array}{c}\text { spin quan } \\
\text { number } \\
\quad \begin{array}{l}\text { w } 05\end{array}\end{array}$ & $\begin{array}{c}\text { concentration } \\
\text { ratio } \\
w 06\end{array}$ \\
\hline $\begin{array}{l}\text { ultra-syn mode } \\
\text { vector } G M 01\end{array}$ & $8.50 \sim 11.10$ & $\begin{array}{c}10^{-10} \times \\
295,300 \mathrm{~km}\end{array}$ & $\begin{array}{c}1.845 \times 10^{33} \\
\mathrm{~kg} \cdot \mathrm{cm}^{-3}\end{array}$ & $\begin{array}{c}1 / 10^{3} \sim \\
1 / 10^{2}\end{array}$ & $\begin{array}{l}0 \quad \text { Higgs } \\
2 \text { graviton }\end{array}$ & 1 \\
\hline $\begin{array}{l}\text { ultra-syn mode } \\
\text { vector } G M 02\end{array}$ & $0.85 \sim 1.11$ & $\begin{array}{c}10^{-9} \times \\
295,300 \mathrm{~km}\end{array}$ & $\begin{array}{c}1.845 \times 10^{30} \\
\mathrm{~kg} \cdot \mathrm{cm}^{-3}\end{array}$ & $\begin{array}{c}1 / 10^{4} \sim \\
1 / 10^{3}\end{array}$ & $\begin{array}{l}0 \quad \text { Higgs } \\
2 \text { graviton }\end{array}$ & $1.5924 \times 10^{-4}$ \\
\hline $\begin{array}{l}\text { ultra-syn mode } \\
\text { vector } G M 03\end{array}$ & $0.085 \sim 0.11$ & $\begin{array}{c}10^{-8} \times \\
295,300 \mathrm{~km}\end{array}$ & $\begin{array}{c}1.845 \times 10^{27} \\
\mathrm{~kg} \cdot \mathrm{cm}^{-3}\end{array}$ & $\begin{array}{c}1 / 10^{5} \sim \\
1 / 10^{4}\end{array}$ & $\begin{array}{l}0 \quad \text { Higgs } \\
2 \text { graviton }\end{array}$ & $\begin{array}{c}0.0000000149 \\
\times 10^{-4}\end{array}$ \\
\hline $\begin{array}{l}\text { ultra-syn mode } \\
\text { vector } G M 04\end{array}$ & $0.0085 \sim 0.011$ & $\begin{array}{c}10^{-7} \times \\
295,300 \mathrm{~km}\end{array}$ & $\begin{array}{c}1.845 \times 10^{24} \\
\mathrm{~kg} \cdot \mathrm{cm}^{-3}\end{array}$ & $\begin{array}{c}1 / 10^{6} \sim \\
1 / 10^{5}\end{array}$ & $\begin{array}{l}0 \quad \text { Higgs } \\
2 \text { graviton }\end{array}$ & $\begin{array}{r}1.44209 \\
\times 10^{-6}\end{array}$ \\
\hline $\begin{array}{l}\text { ultra-syn mode } \\
\text { vector } G M 05\end{array}$ & $\begin{array}{c}0.00085 \sim \\
0.0011\end{array}$ & $\begin{array}{c}10^{-6} \times \\
295,300 \mathrm{~km}\end{array}$ & $\begin{array}{c}1.845 \times 10^{21} \\
\mathrm{~kg} \cdot \mathrm{cm}^{-3}\end{array}$ & $\begin{array}{c}1 / 10^{7} \sim \\
1 / 10^{6}\end{array}$ & $\begin{array}{l}0 \quad \text { Higgs } \\
2 \text { graviton }\end{array}$ & $\begin{array}{c}0.88846 \\
\times 10^{-7}\end{array}$ \\
\hline $\begin{array}{l}\text { ultra-syn mode } \\
\text { vector } G M 06\end{array}$ & $\begin{array}{c}0.000085 \sim \\
0.00011\end{array}$ & $\begin{array}{c}10^{-5} \times \\
295,300 \mathrm{~km}\end{array}$ & $\begin{array}{c}1.845 \times 10^{18} \\
\mathrm{~kg} \cdot \mathrm{cm}^{-3}\end{array}$ & $\begin{array}{c}1 / 10^{8} \sim \\
1 / 10^{7}\end{array}$ & $\begin{array}{l}0 \quad \text { Higgs } \\
2 \text { graviton }\end{array}$ & $\begin{array}{c}0.67682 \\
\times 10^{-8}\end{array}$ \\
\hline $\begin{array}{l}\text { ultra-syn mode } \\
\text { vector } G M 07\end{array}$ & $\begin{array}{c}0.0000085 \sim \\
0.000011\end{array}$ & $\begin{array}{c}10^{-4} \times \\
295,300 \mathrm{~km}\end{array}$ & $\begin{array}{c}1.845 \times 10^{15} \\
\mathrm{~kg} \cdot \mathrm{cm}^{-3}\end{array}$ & $\begin{array}{c}1 / 10^{9} \sim \\
1 / 10^{8}\end{array}$ & $\begin{array}{l}0 \text { Higgs } \\
2 \text { graviton }\end{array}$ & $\begin{array}{r}0.20825 \\
\times 10^{-10}\end{array}$ \\
\hline $\begin{array}{l}\text { ultra-syn mode } \\
\text { vector } G M 08\end{array}$ & $\begin{array}{c}0.00000085 \sim \\
0.0000011\end{array}$ & $\begin{array}{c}10^{-3} \times \\
295,300 \mathrm{~km}\end{array}$ & $\begin{array}{c}1.845 \times 10^{12} \\
\mathrm{~kg} \cdot \mathrm{cm}^{-3}\end{array}$ & $\begin{array}{c}1 / 10^{10} \sim \\
1 / 10^{9}\end{array}$ & $\begin{array}{l}0 \text { Higgs } \\
2 \text { graviton }\end{array}$ & $\begin{array}{r}0.12566 \\
\times 10^{-16}\end{array}$ \\
\hline $\begin{array}{l}\text { ultra-syn mode } \\
\text { vector } G M 09\end{array}$ & $\begin{array}{c}0.000000085 \sim \\
0.00000011\end{array}$ & $\begin{array}{c}10^{-2} \times \\
295,300 \mathrm{~km}\end{array}$ & $\begin{array}{c}1.845 \times 10^{9} \\
\mathrm{~kg} \cdot \mathrm{cm}^{-3}\end{array}$ & $\begin{array}{c}1 / 10^{11} \sim \\
1 / 10^{10}\end{array}$ & $\begin{array}{l}0 \quad \text { Higgs } \\
2 \text { graviton }\end{array}$ & $\begin{array}{c}1.159707 \\
\times 10^{-37}\end{array}$ \\
\hline $\begin{array}{l}\text { ultra-syn mode } \\
\text { vector } G M 10\end{array}$ & $\begin{array}{c}0.0000000085 \sim \\
0.000000011\end{array}$ & $\begin{array}{c}10^{-1} \times \\
295,300 \mathrm{~km}\end{array}$ & $\begin{array}{l}1.845 \times 10^{6} \\
\mathrm{~kg} \cdot \mathrm{cm}^{-3}\end{array}$ & $\begin{array}{c}1 / 10^{12} \sim \\
1 / 10^{11}\end{array}$ & $\begin{array}{l}0 \quad \text { Higgs } \\
2 \text { graviton }\end{array}$ & $\begin{array}{r}0.67546 \\
\times 10^{-46}\end{array}$ \\
\hline $\begin{array}{l}\text { ultra-syn mode } \\
\text { vector } G M 11\end{array}$ & $\begin{array}{l}0.00000000085 \\
\sim 0.0000000011\end{array}$ & $295,300 \mathrm{~km}$ & $\begin{array}{l}1.845 \times 10^{3} \\
\mathrm{~kg} \cdot \mathrm{cm}^{-3}\end{array}$ & $\begin{array}{c}1 / 10^{13} \sim \\
1 / 10^{12}\end{array}$ & $\begin{array}{l}0 \quad \text { Higgs } \\
2 \text { graviton }\end{array}$ & $\begin{array}{l}1.46779 \\
\times 10^{-61}\end{array}$ \\
\hline
\end{tabular}

mode type 001: $\quad w[001]=\left(8.50 \sim 11.10,10^{-10} \times 295,300 \mathrm{~km}, 1.845 \times 10^{33} \mathrm{~kg} \cdot \mathrm{cm}^{-3}, 1 / 10^{3}\right.$ $\left.\sim 1 / 10^{2},\{0,2\}, 1.00000000000\right) \in \Omega$;

mode type 002: $\quad w[001]=\left(0.85 \sim 1.11,10^{-9} \times 295,300 \mathrm{~km}, 1.845 \times 10^{30} \mathrm{~kg} \cdot \mathrm{cm}^{-3}, 1 / 10^{4} \sim\right.$ $\left.1 / 10^{3},\{0,2\}, 1.5924 \times 10^{-4}\right) \in \Omega$;

mode type 003: $\quad w[001]=\left(0.085 \sim 0.11,10^{-8} \times 295,300 \mathrm{~km}, 1.845 \times 10^{27} \mathrm{~kg} \cdot \mathrm{cm}^{-3}, 1 / 10^{5}\right.$ 
$\left.\sim 1 / 10^{4}, 0.70-1.00,0.0000000149 \times 10^{-4}\right) \in \Omega$;

mode type 004: $w[001]=\left(0.0085 \sim 0.011,10^{-7} \times 295,300 \mathrm{~km}, 1.845 \times 10^{24} \mathrm{~kg} \cdot \mathrm{cm}^{-3}, 1 /\right.$ $\left.10^{6} \sim 1 / 10^{5},\{0,2\}, 1.44209 \times 10^{-6}\right) \in \Omega$;

mode type 005: $\quad w[001]=\left(0.00085 \sim 0.0011,10^{-6} \times 295,300 \mathrm{~km}, 1.845 \times 10^{21} \mathrm{~kg} \cdot \mathrm{cm}^{-3}, 1 /\right.$ $\left.10^{7} \sim 1 / 10^{6},\{0,2\}, 0.88846 \times 10^{-7}\right) \in \Omega$;

mode type 006: $\quad w[001]=\left(0.000085 \sim 0.00011,10^{-5} \times 295,300 \mathrm{~km}, 1.845 \times 10^{18} \mathrm{~kg} \cdot \mathrm{cm}^{-3}\right.$, $\left.1 / 10^{8} \sim 1 / 10^{7},\{0,2\}, 0.67682 \times 10^{-8}\right) \in \Omega$;

mode type 007: $\quad w[001]=\left(0.0000085 \sim 0.000011,10^{-4} \times 295,300 \mathrm{~km}, 1.845 \times 10^{15} \mathrm{~kg} \cdot \mathrm{cm}^{-}\right.$ $\left.{ }^{3}, 1 / 10^{9} \sim 1 / 10^{8},\{0,2\}, 0.20825 \times 10^{-10}\right) \in \Omega$;

mode type 008: $w[001]=\left(0.00000085 \sim 0.0000011,10^{-3} \times 295,300 \mathrm{~km}, 1.845 \times 10^{12} \mathrm{~kg}\right.$. $\left.\mathrm{cm}^{-3}, 1 / 10^{10} \sim 1 / 10^{9},\{0,2\}, 0.12566 \times 10^{-16}\right) \in \Omega$;

mode type 009: $\quad w[001]=\left(0.000000085 \sim 0.00000011,10^{-2} \times 295,300 \mathrm{~km}, 1.845 \times 10^{9} \mathrm{~kg}\right.$. $\left.\mathrm{cm}^{-3}, 1 / 10^{11} \sim 1 / 10^{10},\{0,2\}, 1.159707 \times 10^{-37}\right) \in \Omega$;

Table 3 Distrib-Configuration Analysis of Quantum Physic-Sphere Centered on HLX-1 Black Hole

\begin{tabular}{|c|c|c|c|c|c|c|}
\hline & $\begin{array}{l}\text { u-syn mode } \\
01 \text { vect } w\end{array}$ & $\begin{array}{l}\text { u-syn mode } \\
02 \text { vect } w\end{array}$ & $\begin{array}{c}\text { u-syn mode } \\
03 \text { vect } w\end{array}$ & $\begin{array}{l}\text { u-syn mode } \\
04 \text { vect } w\end{array}$ & $\begin{array}{l}\text { u-syn mode } \\
05 \text { vect } w\end{array}$ & $\begin{array}{c}\text { u-syn mode } \\
06 \text { vect } w\end{array}$ \\
\hline $\begin{array}{l}\text { spin net space } \\
\text { SN } 01\end{array}$ & $\begin{array}{c}0.27878203 \\
\times 10^{11}\end{array}$ & & & & & \\
\hline $\begin{array}{l}\text { spin net space } \\
\qquad N 02\end{array}$ & $\begin{array}{c}1.48298008 \\
\times 10^{12}\end{array}$ & $\begin{array}{c}3.49843201 \\
\times 10^{14}\end{array}$ & & & & \\
\hline $\begin{array}{l}\text { spin net space } \\
\qquad N 03\end{array}$ & & $\begin{array}{c}4.23378311 \\
\times 10^{15}\end{array}$ & $\begin{array}{c}4.55278832 \\
\times 10^{17}\end{array}$ & & & \\
\hline $\begin{array}{c}\text { spin net space } \\
\text { SN } 04\end{array}$ & & & $\begin{array}{c}3.28980209 \\
\times 10^{18}\end{array}$ & $\begin{array}{c}0.33247982 \\
\quad \times 10^{20}\end{array}$ & & \\
\hline $\begin{array}{l}\text { spin net space } \\
\qquad N 05\end{array}$ & & & & $\begin{array}{c}1.92811205 \\
\times 10^{21}\end{array}$ & $\begin{array}{c}0.63572768 \\
\quad \times 10^{23}\end{array}$ & \\
\hline $\begin{array}{c}\text { spin net space } \\
\text { SN } 06\end{array}$ & & & & & $\begin{array}{c}0.48927820 \\
\times 10^{24}\end{array}$ & $\begin{array}{c}1.39928310 \\
\times 10^{26}\end{array}$ \\
\hline $\begin{array}{l}\text { spin net space } \\
\text { SN } 07\end{array}$ & & & & & & $\begin{array}{c}2.33878207 \\
\times 10^{27}\end{array}$ \\
\hline $\begin{array}{l}\text { spin net space } \\
\text { SN } 08\end{array}$ & & & & & & \\
\hline $\begin{array}{c}\text { spin net space } \\
\text { SN } 09\end{array}$ & & & & & & \\
\hline $\begin{array}{l}\text { spin net space } \\
\text { SN } 10\end{array}$ & & & & & & \\
\hline $\begin{array}{l}\text { spin net space } \\
\qquad N 11\end{array}$ & & & & & & \\
\hline
\end{tabular}

mode type 0010: $\quad w[001]=\left(0.0000000085 \sim 0.000000011,10^{-1} \times 295,300 \mathrm{~km}, 1.845 \times 10^{6}\right.$ 
$\left.\mathrm{kg} \cdot \mathrm{cm}^{-3}, 1 / 10^{12} \sim 1 / 10^{11},\{0,2\}, 0.67546 \times 10^{-46}\right) \in \Omega$;

mode type 0011: $\quad w[001]=\left(0.00000000085 \sim 0.0000000011,295,300 \mathrm{~km}, 1.845 \times 10^{3} \mathrm{~kg}\right.$. $\left.\mathrm{cm}^{-3}, 1 / 10^{13} \sim 1 / 10^{12},\{0,2\}, 1.46779 \times 10^{-61}\right) \in \Omega$.

Tables 3 (Li Zongcheng, 2019) and 4 (Li Zongcheng, 2019) give the results of the distribution configuration analysis of the quantum Physic-Sphere centered on HLX-1 black hole.

Table 4 Distrib-Configuration Analysis of Quantum Physic-Sphere Centered on HLX-1 Black Hole

\begin{tabular}{|c|c|c|c|c|c|}
\hline & $\begin{array}{l}\text { u-syn mode } \\
07 \text { vect } w\end{array}$ & $\begin{array}{l}\text { u-syn mode } \\
08 \text { vect } w\end{array}$ & $\begin{array}{c}\text { u-syn mode } \\
09 \text { vect } w\end{array}$ & $\begin{array}{l}\text { u-syn mode } \\
10 \text { vect } w\end{array}$ & $\begin{array}{l}\text { u-syn mode } \\
11 \text { vect } w\end{array}$ \\
\hline $\begin{array}{l}\text { spin net space } \\
\qquad S N 01\end{array}$ & & & & & \\
\hline $\begin{array}{l}\text { spin net space } \\
\text { SN } 02\end{array}$ & & & & & \\
\hline $\begin{array}{l}\text { spin net space } \\
\qquad N 03\end{array}$ & & & & & \\
\hline $\begin{array}{l}\text { spin net space } \\
\text { SN } 04\end{array}$ & $\begin{array}{c}5.52918413 \\
\times 10^{25}\end{array}$ & & & & \\
\hline $\begin{array}{l}\text { spin net space } \\
\text { SN } 05\end{array}$ & $\begin{array}{c}1.39928310 \\
\times 10^{26}\end{array}$ & $\begin{array}{c}3.31738172 \\
\times 10^{28}\end{array}$ & & & \\
\hline $\begin{array}{l}\text { spin net space } \\
\text { SN } 06\end{array}$ & $\begin{array}{c}3.39814184 \\
\times 10^{27}\end{array}$ & $\begin{array}{c}0.82842191 \\
\times 10^{29}\end{array}$ & $\begin{array}{c}7.81984101 \\
\times 10^{31}\end{array}$ & & \\
\hline $\begin{array}{l}\text { spin net space } \\
\text { SN } 07\end{array}$ & $\begin{array}{c}0.72984113 \\
\times 10^{28}\end{array}$ & $\begin{array}{c}2.28481981 \\
\times 10^{30}\end{array}$ & $\begin{array}{c}0.72984129 \\
\times 10^{32}\end{array}$ & $\begin{array}{c}0.98118033 \\
\times 10^{34}\end{array}$ & \\
\hline $\begin{array}{l}\text { spin net space } \\
\qquad N 08\end{array}$ & & $\begin{array}{c}7.72948103 \\
\times 10^{31}\end{array}$ & $\begin{array}{c}0.34891285 \\
\times 10^{33}\end{array}$ & $\begin{array}{c}3.48281102 \\
\times 10^{35}\end{array}$ & $\begin{array}{c}4.14891084 \\
\times 10^{37}\end{array}$ \\
\hline $\begin{array}{l}\text { spin net space } \\
\text { SN } 09\end{array}$ & & & $\begin{array}{c}3.55981424 \\
\times 10^{34}\end{array}$ & $\begin{array}{c}4.11817325 \\
\times 10^{36}\end{array}$ & $\begin{array}{c}4.28150125 \\
\times 10^{38}\end{array}$ \\
\hline $\begin{array}{l}\text { spin net space } \\
\qquad N 10\end{array}$ & & & & $\begin{array}{c}0.92841245 \\
\times 10^{37}\end{array}$ & $\begin{array}{c}1.77294810 \\
\times 10^{39}\end{array}$ \\
\hline $\begin{array}{c}\text { spin net space } \\
\text { SN } 11\end{array}$ & & & & & $\begin{array}{c}3.93049134 \\
\times 10^{40}\end{array}$ \\
\hline
\end{tabular}

\section{Ultra-Synergic Dynamical Model for Quantum Grav-Sphere}

Now, based on the previous discussion and study in this series, we will discuss the establishment of the model of the migration and birth-death process of ultra-synergistic dynamics for quantum gravisphere.

In the model established below, $M$ quantum gravi-sources are considered, and all kinds of things attracted to the quantum Physic-Sphere are divided into physical things (simplicity) and mixed things (complexity). A basic aspect of the evolution mode of quantum Physic-Spheres is "everything 
accumulated in or around a certain quantum gravi-source" and its possible trends. Therefore, the transition probability involves the occurrence and disappearance as well as the migration between quantum gravi-regions.

Here, the stochastic model and the average model are proposed and analyzed. For the model of the accumulation of everything, it is necessary to consider the important new characteristics: the migration of physical things between regions of quantum gravi-accumulation can often be regarded as a linear diffusion process with constant mobility between adjacent regions. This model is only an approximate model of "random migration" of physical thing clusters, but it is inappropriate to use this model to discuss the model of mixed things: the migration of mixed thing clusters to another region (e.g., the range controlled by neutron stars between binary stars) can be regarded as different distributions of complex things, and there exists different complex distribution configurations between them. It can be predicted that the migration of mixed things between different quantum gravi-accumulation regions is the non-linear process as non-diffusion (the transfer rate depends on the current complex distribconfiguration), that is, the transfer to non-neighboring regions occurs.

Now, we discuss the establishment of the master equation and mean equation of the migration and birth-death process of the cluster of interacting things in the quantum Physic-Sphere.

It is assumed that there are $P$ clusters of mixed things $\varphi_{\alpha}(\alpha=1,2, \cdots, P)$ and one-dimensional trend spac $\mathscr{M}$ and only focus on the "accumulation mode". Suppose that there are $L$ accumulation regions or ranges $(i=1,2, \cdots, L)$, for example, in an accumulation region, this corresponds to the tendency $i$ of "accumulation in region $i$ ". The distrib-configuration is expressed as a point in C-dimensional distrib-configuration space, where $C=P L: n \equiv\left\{n_{\alpha}\right\} \in \Xi, \alpha=1,2, \cdots, P, i=1,2, \cdots, L$, $n_{\alpha i}$ is the number of basic components of cluster $\varphi_{\alpha}$ accumulated in the quantum gravi-control region $i$.

If the number of simultaneous migrations between clusters is 1 , we introduce the distribution of transition probability $w[k, n]$ from one distrib-configuration $n \in \Xi$ to another $n \equiv\{n+k\} \in \Xi$. Then, use the following symbols:

( $a$ ) The probability $w^{\alpha}{ }_{j i}[k, n]$ of the transition from the quantum gravi-region $i$ to the quantum gravi-region $j(i \neq j)$ in the cluster $\varphi_{\alpha}$ is as follows:

$$
w_{j i}^{\alpha}[k, n]= \begin{cases}n_{\alpha i} \mu_{j i}^{\alpha}(n, k) & \text { when } \quad k=\left\{\cdots 0 \cdots 1_{\alpha j} \cdots 0 \cdots(-1)_{\alpha i} \cdots 0 \cdots\right\} \\ 0 & \text { for all the other } k\end{cases}
$$

the $\mu_{j i}{ }^{\alpha}[n, k]$ here often depends on the corresponding transition probabilities of $n$ and some trend parameters $k$.

( $b$ ) In the region $i$ of quantum gravity, the transition probabilities $w^{\alpha}{ }_{j+}[k, n]$ and $w^{\alpha}{ }_{j-}[k, n]$ of the generation and extinction of the basic constituent units of cluster $\varphi_{a}$ are respectively:

$$
\begin{aligned}
& w_{i+}^{\alpha}[k, n]= \begin{cases}n_{\alpha i} \beta_{i}^{\alpha} & \text { when } \quad k=\left\{\cdots 0 \cdots 1_{\alpha i} \cdots 0 \cdots\right\} \\
0 & \text { for all the other } k\end{cases} \\
& w_{i-}^{\alpha}[k, n]=\left\{\begin{array}{cc}
n_{\alpha i} \delta_{i}^{\alpha}+n_{\alpha i}^{2} \gamma_{i}^{\alpha} & \text { when } \quad k=\left\{\cdots 0 \cdots(-1)_{\alpha i} \cdots 0 \cdots\right\} \\
0 & \text { for all the other } k
\end{array}\right.
\end{aligned}
$$


here, $\quad \beta^{\alpha}{ }_{i}, \delta^{\alpha}{ }_{i}$ and $\gamma^{\alpha}{ }_{i}$ correspond to birth, death and saturation rates, respectively. If it is necessary, the definition formulas ( $329 a, b$ ) can be also consider the generation and extinction rates depending on more complex distrib-configurations.

( $c$ ) The Volterra-Lotka transition probabilities of interaction between two physical things as the gravitational devourer $(\beta)$ and victim $(\delta)$ in the accumulation range are as follows:

$$
w_{i}^{\delta \beta}[k, n]= \begin{cases}n_{\delta i} n_{\beta i} \gamma_{\delta \beta} & \text { when } \quad k=\left\{\cdots 0 \cdots(-1)_{\delta i} \cdots 0 \cdots 1_{\beta i} \cdots 0 \cdots\right\} \\ 0 & \text { for all the other } k\end{cases}
$$

All transition probabilities of $(a) \sim(c)$ introduced by definition are semi-positive definite. In general, the total transition probability is taken as the sum of all distributions ( $328-330)$ :

$$
\begin{aligned}
w[k, n] & =\sum_{i, j=1}^{L} \sum_{\alpha=1}^{P} w_{j i}^{\alpha}[k, n] \\
& +\sum_{i=1}^{L} \sum_{\alpha=1}^{P}\left(w_{i+}^{\alpha}[k, n]+w_{i-}^{\alpha}[k, n]\right)+\sum_{i=1}^{L} \sum_{\delta \beta} w_{i}^{\delta \beta}[k, n]
\end{aligned}
$$

In the last item on the right of formula ( 331$)$, the sum on $(\delta, \beta)$ refers to the corresponding combination of all gravitational devourers and victims. The general form of the master equation of the model can be obtained from ( 331 ):

$$
\frac{d p(n ; t)}{d t}=\left(\frac{\partial p}{\partial t}\right)_{M}(n ; t)+\left(\frac{\partial p}{\partial t}\right)_{B D}(n ; t)+\left(\frac{\partial p}{\partial t}\right)_{V L}(n ; t)
$$

Here, $p(n ; t)$ is the probability of the occurrence of the distrib-configuration $n$ at moment $t$. The three terms on the right side of ( 332 ) refer to the migration, birth-death, and the Volterra-Lotka process, respectively, which are derived from the three distributions of ( 328 - 330 ) and $w[k, n]$ of ( 331 ).

In order to derive the concrete formulas of these three terms, the "transfer operator" $E_{\alpha i}$, which acts on the distrib-configuration function, is introduced :

$$
E_{\alpha i}^{ \pm 1} f\left(n_{11} \cdots n_{\alpha i} \cdots n_{P L}\right)=f\left(n_{11} \cdots\left(n_{\alpha i} \pm 1\right) \cdots n_{P L}\right)
$$

by ( 331 ) and ( $328 \sim 330$ ), and with definitive formula ( 333 ), we can get the concrete formulas of the three terms on the right side of the master equation ( 332 ) as follows:

$$
\begin{aligned}
& \left(\frac{\partial p}{\partial t}\right)_{M}(n ; t)=\sum_{i, j=1}^{L} \sum_{\alpha=1}^{P}\left(E_{\alpha i} E_{\alpha j}^{-1}-1\right)\left[n_{\alpha i} \mu_{j i}^{\alpha}(n, k) p(n ; t)\right] \\
& \left(\frac{\partial p}{\partial t}\right)_{B D}(n ; t)=\sum_{i=1}^{L} \sum_{\alpha=1}^{P}\left\{\left(E_{\alpha j}^{-1}-1\right)\left[n_{\alpha i} \beta_{i}^{\alpha} p(n ; t)\right]+\left(E_{\alpha i}-1\right)\left[\left(n_{\alpha i} \delta_{\alpha}^{i}+n_{\alpha i}^{2} \gamma_{i}^{\alpha}\right) p(n ; t)\right]\right. \\
& \left(\frac{\partial p}{\partial t}\right)_{V L}(n ; t)=\sum_{i=1}^{L} \sum_{\delta, \beta}\left(E_{\delta i} E_{\alpha j}^{-1}-1\right)\left[n_{\delta i} n_{\beta i} \gamma_{\delta \beta} p(n ; t)\right]
\end{aligned}
$$

From the master equation ( 332 ), the following mean equation can be derived directly: 


$$
<n_{\gamma k}>_{t}=\sum_{n} n_{\gamma k} p(n ; t)
$$

For this purpose, $n_{\gamma k}$ is multiplied by ( 332 ) and the sum is taken on all distrib-configurations $n$. Using the transition formula:

$$
n_{\gamma k} E_{\alpha i} E_{\alpha j}^{-1} f(n) \equiv E_{\alpha i} E_{\alpha j}^{-1}\left(n_{\gamma k}-\delta_{\gamma \alpha} \delta_{k i}+\delta_{\gamma \alpha} \delta_{k j}\right) f(n)
$$

so specific results are obtained from ( 333 ):

$$
\begin{aligned}
\frac{d<n_{\gamma k}>_{t}}{d t} & =\left[\sum_{i=1}^{L}<n_{\gamma} \mu_{k i}^{\gamma}(n, k)>_{t}-\sum_{j=1}^{L}<n_{\gamma k} \mu_{j k}^{\gamma}(n, k)>_{t}\right] \\
& +\left[\left(\beta_{k}^{\gamma}-\delta_{k}^{\gamma}\right)<n_{\gamma k}>_{t}-\gamma_{k}^{\gamma}<n_{\gamma k}^{2}>_{t}\right] \\
& +\left[\sum_{\delta} v_{\delta \gamma}<n_{\delta k} n_{\gamma k}>_{t}-\sum_{\beta} v_{\gamma \beta}<n_{\gamma k} n_{\beta k}>_{t}\right]
\end{aligned}
$$

The three items on the right of formula ( 339 ) refer to the process of migration, birth-death, and gravitational devourer - victim. In the last item, the first sum extends to the species of the victim $\delta$ corresponding to the gravitational devourer $\gamma$, while the second sum extends to the gravitational deviourer $\beta$ which coexists with the victim.

For $p(n, t)$ with only one spike distribution, the exact equation ( 339 ) can be approximated to a closed system of equations with an average value $\left\langle n_{\gamma k}>_{t} \equiv \tilde{n}_{\gamma k}\right.$ :

$$
\begin{aligned}
\frac{d \tilde{n}_{\gamma k}}{d t} & =\left[\sum_{i=1}^{L} \tilde{n}_{\gamma i} \mu_{k i}^{\gamma}(n, k)-\sum_{j=1}^{L} \tilde{n}_{\gamma k} \mu_{j k}^{\gamma}(n, k)\right] \\
& +\left[\left(\beta_{k}^{\gamma}-\delta_{k}^{\gamma}\right) \tilde{n}_{\gamma k}-\gamma_{k}^{\gamma} \tilde{n}_{\gamma k}^{2}\right]+\left[\sum_{\delta} v_{\delta \gamma} \tilde{n}_{\delta k} \tilde{n}_{\gamma k}-\sum_{\beta} v_{\gamma \beta} \tilde{n}_{\gamma k} \tilde{n}_{\beta k}\right]
\end{aligned}
$$

We can also get the equation of variance in the same form, which will be discussed below.

\section{Joint-Varying Equivalence from Scalable Parity of Mass}

In order to improve and expand general relativity and quantum physics, we should now

( $A$ ) consider the extreme spatiotemporal environment in the universe;

( $B$ ) consider the evolution of a gravitational field between a homogeneous or disordered state and an inhomogeneous or ordered state.

For gravitational mass, we consider the following factors:

( $a$ ) during the observation period, there is a very high acceleration of gravity, which is restricted by the photon acceleration in the extreme environment of cosmic space-time (rather than the invariance of the speed of light in vacuum);

( $b$ ) during the observation period, there is a very high acceleration evolution, which is restricted by the evolution acceleration in the extreme environment of cosmic space-time (it may offset the 
sensitivity feeling of the observer from the heterogeneity on gravitational fields);

( $c$ ) during the observation period, there is no other mechanism that leads to changes in masses $M$ and $m$, except for the various effects of spatiotemporal complexities caused by the extremely high gravitational acceleration and the extremely high evolutionary acceleration.

In the full-view space-time $R_{\text {com }}(u, \Sigma, t)$, the gravitational action of the analytical node on coordinate $u_{i}$ with non-equilibrium statistical mass $m_{\eta, i}$, for the analytical node on coordinate $u$ with non-equilibrium statistical mass $m_{\eta}$, should be

$$
F_{\text {com }}=-G_{\text {com }} \frac{m_{\eta} m_{\eta, i}}{u_{i}^{3}} u_{i}=-G_{c o m} \frac{m_{\eta} m_{\eta, i}}{\left|\gamma-\gamma_{i}\right|^{3}}\left(\gamma-\gamma_{i}\right),
$$

where $G_{c o m}$ can be called the joint-varying gravitational constant, where $m_{\eta}$ can be called the jointvarying gravitational mass, $u_{i}=\left|\gamma-\gamma_{i}\right|$. In the continuous distribution of matter, there should be

$$
F_{c o m}(\gamma, t)=-G_{c o m} m_{\eta} \iint \frac{1}{\left|\gamma-\gamma^{\prime}\right|} \rho_{\eta}\left(\gamma^{\prime}\right)\left(\gamma-\gamma^{\prime}\right) d^{3} \gamma^{\prime} d t
$$

Using the operation of vector $\nabla \frac{1}{u}=-\frac{u}{u^{3}}$, where $u=\gamma-\gamma^{\prime}, u=|u|, \nabla$ is the nabla (or del) operator for the joint-varying node $\gamma: \nabla=i \frac{\partial}{\partial \gamma_{x}}+j \frac{\partial}{\partial \gamma_{y}}+k \frac{\partial}{\partial \gamma_{z}}$. Formula ( 341 ) is written into $F_{\text {com }}=-m_{\eta} \nabla \varphi_{c o m}$, where

$$
\varphi_{c o m} \equiv-\iint \frac{1}{\left|\gamma-\gamma^{\prime}\right|} G_{c o m} \rho_{\eta}\left(\gamma^{\prime}\right) d^{3} \gamma^{\prime} d t
$$

is the joint-varying gravitational potential from the continuous field source, $\rho_{\eta}\left(\gamma^{\prime}\right)$ is the density of non-equilibrium matter on the coordinate $\gamma^{\prime}$ in a full-view space-time, $\int d^{3} \gamma^{\prime}$ is the joint- varying integral for the 3-dimensional system of the source.

By the motion-like gauge in the full-view measuring, it is from the gravitational mass to derive the motion-like gravitational mass with evolution course :

$$
\begin{aligned}
& F=G \frac{m M}{R^{2}} \rightarrow F_{m o v}=G \frac{m_{m o v} M_{m o v}}{R_{m o v}^{2}} \\
& \rightarrow F_{m o v}=G m \frac{1}{\sqrt{1-\alpha_{\eta, m}^{2}}}\left(1-\left(\frac{d v_{m}}{d t}\right)^{2} /\left(\frac{d c_{\lambda}}{d t}\right)^{2}\right)^{-1 / 2} M \frac{1}{\sqrt{1-\alpha_{\eta, M}^{2}}} / R_{m o v}^{2}
\end{aligned}
$$

where

$$
\alpha_{m}=\frac{V_{\sigma, m}}{V_{\gamma}} \rightarrow \alpha_{\eta, m}=\frac{d V_{\sigma, m}}{d t} / \frac{d V_{\gamma}}{d t}, \quad \alpha_{M}=\frac{V_{\sigma, M}}{V_{\gamma}} \rightarrow \alpha_{\eta, M}=\frac{d V_{\sigma, M}}{d t} / \frac{d V_{\gamma}}{d t}
$$


$R_{\text {mov }}^{2}=\left\{R\left[1-\left(\frac{d v_{M}}{d t}\right)^{2} /\left(\frac{d c_{\lambda}}{d t}\right)^{2}\right]^{1 / 2}\right\}^{2}=R^{2}\left[1-\left(\frac{d v_{M}}{d t}\right)^{2} /\left(\frac{d c_{\lambda}}{d t}\right)^{2}\right]$

$\frac{d V_{\sigma, m}}{d t}$ is the evolution accelerated rate of mass $m, \frac{d V_{\sigma, M}}{d t}$ is the evolution accelerated rate of mass $M, \frac{d V_{\gamma}}{d t}$ is the maximum effective rate of evolving acceleration under the restriction of the horizon (such as the evolution accelerated rate of a group of photons from $\gamma$-ray bursts), $\frac{d v_{m}}{d t}$ is the gravitational acceleration of mass $m, \frac{d v_{M}}{d t}$ is the gravitational acceleration of mass $M$,

$c_{\lambda}$ is the cascade evolving rate of bifurcate-chaotic photons derived by the sufficient proof in the foregoing paper of this series. By the Feigenbaum's geometric proportion of bifurcate spacing $\Delta_{n} / \Delta_{n+1}=\delta=4.669201609 \ldots \ldots$, the cascade evolving rate was given in the foregoing paper of this series for the $N$ stage bifurcate-chaotic photon in general env., extreme evn. and vacuum env.

$$
c_{\lambda}=\eta_{0} c+\eta_{1} 2 c+\frac{1}{\delta} \eta_{1} 3 c+\frac{1}{\delta^{2}} \eta_{1} 4 c+\cdots+\frac{1}{\delta^{N-1}} \eta_{1}(1+N) c=c\left[\eta_{0}+\sum_{n=1}^{N} \frac{1}{\delta^{n-1}} \eta_{1}(1+n)\right]
$$

where $\delta$ is Feigenbaum constant; $\eta_{\mathrm{o}}$ is the proportion of un-bifurcated period $\left[t_{\mathrm{o}}, t_{1}\right]$ in a specific period $\left[t_{0}, t\right]$ of evolution, and $0 \leq \eta_{0}<1 ; \eta_{1}$ is the proportion of the 1st stage bifurcated period $\left[t_{1}, t\right.$ 2] in $\left[t_{\mathrm{o}}, t\right] . c_{\lambda}$ has itself limit value at different values. For example, when $\delta=4.6692$, the limit of $c_{\lambda}$ is about $c_{N}=2.99 c$. Generally, $c_{\lambda}$ varies between $c$ and $2.99 c$ with the evolution of the nonlinear non-equilibrium photon. That is, when $\delta=4.6692, c_{\lambda}$ is within $c=2.99792 \times 10^{10} \mathrm{~cm} / \mathrm{s} \sim 2.99 c=$ $2.99 \times 2.99792 \times 10^{10} \mathrm{~cm} / \mathrm{s}$.

So as to obtain

$$
F_{m o v}=G m \frac{1}{\sqrt{1-\alpha_{\eta, m}^{2}}}\left(1-\left(\frac{d v_{m}}{d t}\right)^{2} /\left(\frac{d c_{\lambda}}{d t}\right)^{2}\right)^{-1 / 2} M \frac{1}{\sqrt{1-\alpha_{\eta, m}^{2}}} / R^{2}\left[1-\left(\frac{d v_{M}}{d t}\right)^{2} /\left(\frac{d c_{\lambda}}{d t}\right)^{2}\right]
$$

By the evolution-like gauge in the full-view measuring, it is from the gravitational mass to derive the evolution-like gravitational mass with evolution course :

$$
\begin{aligned}
& F=G \frac{m M}{R^{2}} \rightarrow F_{d e v}=G \frac{m_{d e v} M_{d e v}}{R_{d e v}^{2}} \\
& \rightarrow F_{d e v}=G m \frac{1}{\sqrt{1-\beta_{\eta, m}^{2}}}\left(1-\left(\frac{d V_{\sigma, m}}{d t}\right)^{2} /\left(\frac{d V_{\gamma}}{d t}\right)^{2}\right)^{-1 / 2} M\left(1-\left(\frac{d V_{\sigma, M}}{d t}\right)^{2} /\left(\frac{d V_{\gamma}}{d t}\right)^{2}\right)^{-1 / 2} / R_{d e v}^{2}
\end{aligned}
$$

where

$$
\beta=\frac{v}{c} \rightarrow \beta_{\eta, m}=\frac{d v_{m}}{d t} / \frac{d c_{\lambda}}{d t},
$$


$\frac{d V_{\sigma, m}}{d t}$ is the evolution accelerated rate of mass $m, \frac{d V_{\sigma, M}}{d t}$ is the evolution accelerated rate of mass $M, \frac{d V_{\gamma}}{d t}$ is the maximum effective rate of evolving acceleration under the restriction of the horizon (such as the evolution accelerated rate of a group of photons from $\gamma$-ray bursts),

$\frac{d v_{m}}{d t}$ is the gravitational acceleration of mass $m, \frac{d v_{M}}{d t}$ is the gravitational acceleration of mass $M$, $\frac{d c_{\lambda}}{d t}$ is the acceleration of the photon in the extreme spatiotemporal env (which is free from the invariance of the speed of light in a vacuum),

$$
R_{d e v}^{2}=\left\{R\left[1-\left(\frac{d V_{\sigma, M}}{d t}\right)^{2} /\left(\frac{d V_{\gamma}}{d t}\right)^{2}\right]^{-1 / 2}\right\}^{2}=R^{2}\left[1-\left(\frac{d V_{\sigma, M}}{d t}\right)^{2} /\left(\frac{d V_{\gamma}}{d t}\right)^{2}\right]^{-1}
$$

So as to obtain

$$
F_{d e v}=G m \frac{1}{\sqrt{1-\beta_{\eta}^{2}}}\left(1-\left(\frac{d V_{\sigma, m}}{d t}\right)^{2} /\left(\frac{d V_{\gamma}}{d t}\right)^{2}\right)^{-1 / 2} M \sqrt{1-\left(\frac{d V_{\sigma, M}}{d t}\right)^{2} /\left(\frac{d V_{\gamma}}{d t}\right)^{2}} / R_{d e v}^{2}
$$

By the motion-like gauge in the full-view measuring, it is from the inertial mass to derive the motion-like inertial mass with evolution course :

$$
\begin{aligned}
F=m \frac{d^{2} r}{d t^{2}} & \rightarrow F_{m o v}=m_{m o v} \frac{d^{2} r_{m o v}}{d t^{2}} \\
& \rightarrow F_{m o v}=m \frac{1}{\sqrt{1-\alpha_{\eta, m}^{2}}}\left(1-\left(\frac{d v_{m}}{d t}\right)^{2} /\left(\frac{d c_{\lambda}}{d t}\right)^{2}\right)^{-1 / 2} \frac{d^{2} r_{m o v}}{d t^{2}}
\end{aligned}
$$

where

$$
\alpha_{m}=\frac{V_{\sigma, m}}{V_{\gamma}} \rightarrow \alpha_{\eta, m}=\frac{d V_{\sigma, m}}{d t} / \frac{d V_{\gamma}}{d t}, \quad r_{m o v}=r\left[1-\left(\frac{d v_{m}}{d t}\right)^{2} /\left(\frac{d c_{\lambda}}{d t}\right)^{2}\right]^{-1 / 2}
$$

$\frac{d v_{m}}{d t}$ is the gravitational acceleration of mass $m$,

$\frac{d V_{\sigma, m}}{d t}$ is the evolution accelerated rate of mass $m$,

$\frac{d V_{\gamma}}{d t}$ is the maximum effective rate of evolving acceleration under the restriction of the horizon (such as the evolution accelerated rate of a group of photons from $\gamma$-ray bursts),

$\frac{d c_{\lambda}}{d t}$ is the acceleration of the photon in the extreme spatiotemporal env (which is free from the invariance of the speed of light in a vacuum), 
So as to obtain

$$
F_{\text {mov }}=m \frac{1}{\sqrt{1-\alpha_{\eta, m}^{2}}}\left(1-\left(\frac{d v_{m}}{d t}\right)^{2} /\left(\frac{d c_{\lambda}}{d t}\right)^{2}\right)^{-1 / 2} \frac{d^{2}}{d t^{2}}\left\{\left(r-v_{M} t\right)\left[1-\left(\frac{d v_{M}}{d t}\right)^{2} /\left(\frac{d c_{\lambda}}{d t}\right)^{2}\right]^{-1 / 2}\right\}
$$

By the evolution-like gauge in the full-view measuring, it is from the inertial mass to derive the evolution-like inertial mass with motion process :

$$
\begin{aligned}
F=m \frac{d^{2} \zeta}{d t^{2}} & \rightarrow F_{d e v}=m_{d e v} \frac{d^{2} \zeta_{d e v}}{d t^{2}} \\
\rightarrow F_{d e v} & =m \frac{1}{\sqrt{1-\beta_{\eta, m}^{2}}}\left(1-\left(\frac{d V_{\sigma, m}}{d t}\right)^{2} /\left(\frac{d V_{\gamma}}{d t}\right)^{2}\right)^{-1 / 2} \frac{d^{2} \zeta_{d e v}}{d t^{2}}
\end{aligned}
$$

where

$$
\begin{aligned}
& \beta=\frac{v}{c} \rightarrow \beta_{\eta, m}=\frac{d v_{m}}{d t} / \frac{d c_{\lambda}}{d t}, \quad \zeta_{d e v}=\zeta\left[1-\left(\frac{d V_{\sigma, M}}{d t}\right)^{2} /\left(\frac{d V_{\gamma}}{d t}\right)^{2}\right]^{-1 / 2} \\
& \frac{d V_{\sigma, m}}{d t} \text { is the evolution accelerated rate of mass } m, \frac{d V_{\gamma}}{d t} \text { is the maximum effective rate of }
\end{aligned}
$$

evolving acceleration under the restriction of the horizon (such as the evolution accelerated rate of a group of photons from $\gamma$-ray bursts),

$\frac{d v_{m}}{d t}$ is the gravitational acceleration of mass $m, \frac{d c_{\lambda}}{d t}$ is the acceleration of the photon in the extreme spatiotemporal env (which is free from the invariance of the speed of light in a vacuum),

So as to obtain

$$
F_{d e v}=m \frac{1}{\sqrt{1-\beta_{\eta}^{2}}}\left(1-\left(\frac{d V_{\sigma, m}}{d t}\right)^{2} /\left(\frac{d V_{\gamma}}{d t}\right)^{2}\right)^{-1 / 2} \frac{d^{2}}{d t^{2}}\left\{\left(\zeta-V_{\sigma, m} t\right)\left[1-\left(\frac{d V_{\sigma, m}}{d t}\right)^{2} /\left(\frac{d V_{\gamma}}{d t}\right)^{2}\right]^{-1 / 2}\right\}
$$

Now, for the evolution (and its measurement and gauge) in the process of evolution and the evolution (and its measurement and gauge), the first basic hypothesis on the spatiotemporal relation of generalized joint-varying relativity can be established, its contents is summarized as follows:

In the case for the accelerate-rate of change to approximate the accelerate-speed of the photon (and a group of photons) in the extreme spatiotemporal environment (the invariance of light speed is limited only in vacuum), a non-inertial joint-varying system with the accelerate-rate of change not to be zero, and a non-inertial joint-varying system with any gravitational field (the very high accelerate-rate of change between the homogeneity and heterogeneity should eliminate any difference by the way more than the feeling sensitivity limit of observers ), are indistinguishable for observers.

This property can be called the equivalence of non-inertial joint-varying system. In the future, this basic hypothesis can be elevated to a basic principle. 


\section{With Binary Star of Neutron Star and Black Hold as Example}

On the theoretical basis, classical and modern physics is basically set up under the restriction of the scope the earth's space-time (or the solar space-time), failed to give full consideration on those phenomena and problems which is the "normal" in the large scale scope of the cosmic space-time but the seemingly incredible "extreme" in the range of the earth's space-time. On the abnormal phenomena such as the initial inflation, the accelerating expansion of the universe, the gigantic jet of extragalactic compact radio sources, the explosion of supernovas, the giant red-shift of quasars, $\gamma$ ray bursts, the amazing phagocytosis of massive black hole and so on, we see the more and more diverse, increasingly complex world.

In order to overcome the limitations of the "physics" from the time and space of the earth-sun system, let us take the binary star in the extragalactic system as example, for example, taking a binary star consisting of a neutron with its mass in $M_{n-\text { star }}=3.282 \times 10^{30} \mathrm{~kg}$ and a black hold with its mass in $M_{B H}=19.890 \times 10^{30} \mathrm{~kg}$ as example, the joint-varying equivalence of mass under the full-view gauge can be explained.

Under the semi-classical approximation (non-quantization), it is aimed at the black hold to add the integral matter field in the action quantity of a classical gravity, so to obtain the part of quantum :

$$
W=W_{c l}+\Gamma, \quad W_{c l} \text { is the classical gravitational field }
$$

In the degeneration of spherical symmetry, $W_{c l}$ is taken as the gravity of dilaton

$$
W_{c l}=-\frac{1}{4 G} \int_{M^{2}}\left[r^{2} R+2(\nabla r)^{2}+2 U(r)\right] \sqrt{\xi} d^{2} z-\frac{1}{2 G} \int_{\partial M^{2}} r^{2} k
$$

where $r^{2}(z)$ play a action of dilaton field, $\xi$ is the gauge of 2-dimensional effective space $M^{2}$, dilaton potential is

$$
U(r)=1-\frac{Q^{2}}{r^{2}}, \quad Q \text { is electric charge }
$$

In a self-consistent processing, the effective quantum acting-quantity $\Gamma$ for a singe loop to contribute should also be obtained by the 4-dimensional matter field via the degradation of spherical symmetry.

Taking into account the quantum effects of the single loop of black holes, it is below that the classic geometry of the black hole is modified by quantum corrections (formula ( 348 )) of action quantity, and the formula ( 349 ) is used to obtain the variation of the metric

$$
G_{\alpha \beta}=-T_{\alpha \beta}, \quad T_{\alpha \beta}=\frac{G}{24 \pi}\left\{2 \nabla_{\alpha} \nabla_{\beta} \psi-\partial_{\alpha} \psi \partial_{\beta} \psi-\eta_{\alpha \beta}\left[2 R-\frac{1}{2}(\nabla \psi)^{2}\right]\right\}
$$

where $G_{\alpha \beta}$ is obtained by the variation of the metric $\eta_{\alpha \beta}$

$$
G_{\alpha \beta} \equiv-2 r \nabla_{\alpha} \nabla_{\beta} r+\eta_{\alpha \beta}\left[\square r^{2}-(\nabla r)^{2}-U\right]=0
$$

When the gravitational accelerate-speed of a neutron star is $\frac{d v_{m}}{d t}=\frac{d}{d t}\left(v_{m}>0.650 c\right)$, the 
accelerate-speed (which is free from the invariance of the speed of light in a vacuum) of photon in the extreme spatiotemporal environment is $\frac{d c_{\lambda}}{d t}=\frac{d}{d t}\left(c_{\lambda}>0.999 c\right)$, on the joint-varying gravitational mass with the evolution course, there should be the following result :

$$
\begin{aligned}
& m \frac{1}{\sqrt{1-\alpha_{\eta, m}^{2}}}\left(1-\left(\frac{d v_{m}}{d t}\right)^{2} /\left(\frac{d c_{\lambda}}{d t}\right)^{2}\right)^{-1 / 2} \\
& =3.282 \times 10^{30} \mathrm{~kg} \times \frac{1}{\sqrt{1-\alpha_{\eta, m}^{2}}}\left(1-\left(\frac{d}{d t}\left(v_{m}>0.650 c\right)\right)^{2} /\left(\frac{d}{d t}\left(c_{\lambda}>0.999 c\right)\right)^{2}\right)^{-1 / 2} \\
& M \frac{1}{\sqrt{1-\alpha_{\eta, m}^{2}}}=19.890 \times 10^{30} \mathrm{~kg} \times \frac{1}{\sqrt{1-\alpha_{\eta, m}^{2}}} \\
& R^{2}\left[1-\left(\frac{d v_{M}}{d t}\right)^{2} /\left(\frac{d c_{\lambda}}{d t}\right)^{2}\right]=(0.550 \mathrm{~km})^{2}\left[1-\left(\frac{d}{d t}\left(v_{m}>0.650 c\right)\right)^{2} /\left(\frac{d}{d t}\left(c_{\lambda}>0.999 c\right)\right)^{2}\right]
\end{aligned}
$$

When the evolution accelerate rate of a neutron star is $\frac{d V_{\sigma, m}}{d t}=\frac{d}{d t}\left(V_{\sigma, m}>\frac{d}{d t} 1598.928 \times 10^{24}\right)$, the evolution accelerate rate of a black hold is $\frac{d V_{\sigma, M}}{d t}=\frac{d}{d t}\left(V_{\sigma, M}>\frac{d}{d t} 2982.373 \times 10^{24}\right)$, the maximum effective rate of evolving acceleration under the restriction of the horizon (such as the evolution accelerated rate of a group of photons from $\gamma$-ray bursts $)$ is $\frac{d V_{\gamma}}{d t}=\frac{d}{d t}\left(V_{\gamma}>\frac{d}{d t} 3683.84995 \times 10^{24}\right)$, on the joint-varying gravitational mass with motion processes, there is the following result :

$$
\begin{aligned}
& M \sqrt{1-\left(\frac{d V_{\sigma, M}}{d t}\right)^{2} /\left(\frac{d V_{\gamma}}{d t}\right)^{2}} \\
& =19.890 \times 10^{30} \mathrm{~kg} \frac{1}{\sqrt{1-\beta_{\eta}^{2}}}\left(1-\left(\frac{d}{d t}\left(V_{\sigma, M}>\frac{d}{d t} 2982.373 \times 10^{24}\right)\right)^{2} /\left(\frac{d}{d t}\left(V_{\gamma}>\frac{d}{d t} 3683.84995 \times 10^{24}\right)\right)^{2}\right)^{1 / 2}
\end{aligned}
$$

Table 5, Table 6, and Table 7 show the ratio of gravity to evolution accelerate rate (the factor of multiple-gauge transformation). By comparison, we can further see the physical effect of spatiotemporal complexity.

Table 8 and Table 9 show the ratio of mass, the ratio of motion-accelerating speed and the ratio of evolution-accelerating rate. By comparison, we can further see the physical effect of spatiotemporal complexity. 
Table 5 Relation (A) : Ratio of Gravity to Evolution Accelerate rate

\begin{tabular}{|c|c|c|c|c|c|c|}
\hline$\frac{d V_{\sigma, M}}{d t} / \frac{d V_{\gamma}}{d t}$ & 0.50 & 0.50 & 0.50 & 0.50 & 0.50 & 0.50 \\
\hline$\frac{d V_{\sigma, m}}{d t} / \frac{d V_{\gamma}}{d t}$ & 0.00 & 0.01 & 0.10 & 0.50 & 0.75 & 0.80 \\
\hline$F_{m c}$ & $1.1150 F$ & $1.1182 F$ & $1.1206 F$ & $1.2432 F$ & $1.7149 F$ & $1.8587 F$ \\
\hline$\frac{d V_{\sigma, M}}{d t} / \frac{d V_{\gamma}}{d t}$ & 0.50 & 0.50 & 0.50 & 0.50 & 0.50 & 0.50 \\
\hline$\frac{d V_{\sigma, m}}{d t} / \frac{d V_{\gamma}}{d t}$ & 0.85 & 0.90 & 0.95 & 0.98 & 0.99 & 0.998 \\
\hline$F_{m c}$ & $2.1162 F$ & $2.5578 F$ & $3.5713 F$ & $5.6029 F$ & $7.9042 F$ & $17.6393 F$ \\
\hline
\end{tabular}

Table 6 Relation (B) : Ratio of Gravity to Evolution Accelerate rate

\begin{tabular}{|c|c|c|c|c|c|c|}
\hline$\frac{d V_{\sigma, M}}{d t} / \frac{d V_{\gamma}}{d t}$ & 0.80 & 0.80 & 0.80 & 0.80 & 0.80 & 0.80 \\
\hline$\frac{d V_{\sigma, m}}{d t} / \frac{d V_{\gamma}}{d t}$ & 0.00 & 0.01 & 0.10 & 0.50 & 0.75 & 0.80 \\
\hline$F_{m c}$ & $1.6670 F$ & $1.6696 F$ & $1.6753 F$ & $1.8587 F$ & $2.5638 F$ & $2.7789 F$ \\
\hline$\frac{d V_{\sigma, M}}{d t} / \frac{d V_{\gamma}}{d t}$ & 0.80 & 0.80 & 0.80 & 0.80 & 0.80 & 0.80 \\
\hline$\frac{d V_{\sigma, m}}{d t} / \frac{d V_{\gamma}}{d t}$ & 0.85 & 0.90 & 0.95 & 0.98 & 0.99 & 0.998 \\
\hline$F_{m c}$ & $3.1638 F$ & $3.8241 F$ & $5.3394 F$ & $8.3767 F$ & $11.8174 F$ & $26.3719 F$ \\
\hline
\end{tabular}

Table 7 Relation (D) : Ratio of Gravity to Evolution Accelerate rate

\begin{tabular}{|c|c|c|c|c|c|c|}
\hline$\frac{d V_{\sigma, M}}{d t} / \frac{d V_{\gamma}}{d t}$ & 0.99 & 0.99 & 0.99 & 0.99 & 0.99 & 0.99 \\
\hline$\frac{d V_{\sigma, m}}{d t} / \frac{d V_{\gamma}}{d t}$ & 0.00 & 0.01 & 0.10 & 0.50 & 0.75 & 0.80 \\
\hline$F_{m c}$ & $7.0890 F$ & $7.0914 F$ & $7.1244 F$ & $7.9042 F$ & $10.9029 F$ & $11.8174 F$ \\
\hline$\frac{d V_{\sigma, M}}{d t} / \frac{d V_{\gamma}}{d t}$ & 0.99 & 0.99 & 0.99 & 0.99 & 0.99 & 0.99 \\
\hline$\frac{d V_{\sigma, m}}{d t} / \frac{d V_{\gamma}}{d t}$ & 0.85 & 0.90 & 0.95 & 0.98 & 0.99 & 0.998 \\
\hline$F_{m c}$ & $13.4542 F$ & $16.2622 F$ & $22.7061 F$ & $35.6222 F$ & $50.2539 F$ & $112.1480 F$ \\
\hline
\end{tabular}


Table 8 Ratio of Mass, Ratio of Motion-Acc Speed and Ratio of Evolution-Acc rate

\begin{tabular}{|c|c|c|c|c|c|c|}
\hline$\frac{d v}{d t} / \frac{d c^{*}}{d t}$ & 0.50 & 0.50 & 0.50 & 0.50 & 0.50 & 0.50 \\
\hline$\frac{d V_{\sigma}}{d t} / \frac{d V_{\gamma}}{d t}$ & 0.00 & 0.01 & 0.10 & 0.50 & 0.75 & 0.80 \\
\hline$M_{m d} / M$ & 1.1150 & 1.1182 & 1.1206 & 1.2432 & 1.7149 & 1.8587 \\
\hline$\frac{d v}{d t} / \frac{d c^{*}}{d t}$ & 0.50 & 0.50 & 0.50 & 0.50 & 0.50 & 0.50 \\
\hline$\frac{d V_{\sigma}}{d t} / \frac{d V_{\gamma}}{d t}$ & 0.85 & 0.90 & 0.95 & 0.98 & 0.99 & 0.998 \\
\hline$M_{m d} / M$ & 2.1162 & 2.5578 & 3.5713 & 5.6029 & 7.9042 & 17.6393 \\
\hline
\end{tabular}

Table 9 Ratio of Mass, Ratio of Motion-Acc Speed and Ratio of Evolution-Acc rate

\begin{tabular}{|c|c|c|c|c|c|c|}
\hline$\frac{d v}{d t} / \frac{d c^{*}}{d t}$ & 0.99 & 0.99 & 0.99 & 0.99 & 0.99 & 0.99 \\
\hline$\frac{d V_{\sigma}}{d t} / \frac{d V_{\gamma}}{d t}$ & 0.00 & 0.01 & 0.10 & 0.50 & 0.75 & 0.80 \\
\hline$M_{m d} / M$ & 7.0890 & 7.0912 & 7.1244 & 7.9042 & 10.9029 & 11.8174 \\
\hline$\frac{d v}{d t} / \frac{d c^{*}}{d t}$ & 0.99 & 0.99 & 0.99 & 0.99 & 0.99 & 0.99 \\
\hline$\frac{d V_{\sigma}}{d t} / \frac{d V_{\gamma}}{d t}$ & 0.85 & 0.90 & 0.95 & 0.98 & 0.99 & 0.998 \\
\hline$M_{m d} / M$ & 13.4542 & 16.2622 & 22.7061 & 35.6222 & 50.2539 & 112.1480 \\
\hline
\end{tabular}

\section{Acknowledgements}

I would like to express my sincere gratitude to the domestic and foreign researchers, my colleagues and relatives. Without their enlightening instruction, impressive kindness and help, I could not have completed my work.

\section{References}

[1] A. U., Abeysekara et al, Searching for dark matter sub-structure with HAWC, Journal of Cosmology and Astroparticle Physics, 07(2019)022

[2] Ade P A R, Aghanim N, A R Mitage-Caplan C, et al. (Planck Collaboration). Planck 2013 results. XXII. Constraints on inflation[J /OL]. arXiv: 1303. 5082[astro - ph. CO].

[3] N. Agafonova et al, Measurement of the cosmic ray muon flux seasonal variation with the OPERA detector, Journal of Cosmology and Astroparticle Physics, 10(2019)003

[4] A. Challinor et al, Exploring cosmic origins with CORE: Gravitational lensing of the CMB, Journal of 
Cosmology and Astroparticle Physics, 04(2018)018

[5] V. Anastassopoulos et al, Improved search for solar chameleons with a GridPix detector at CAST, Journal of Cosmology and Astroparticle Physics, 01(2019)032

[6] M. Tegmark et al. (SDSS collaboration), Cosmological Parameters from SDSS and WMAP, Phys. Rev. D69 103501 (2004).

[7] D. N. Spergel et al. (WMAP collaboration), First year Wilkinson Microwave Anisotropy Probe (WMAP) observations: determination of cosmological parameters, Astrophys. J. Suppl. 148175 (2003).

[8] R. Rebolo et al. (VSA collaboration), Cosmological parameter estimation using Very Small Array data out to 1 $=1500$, Monthly Notices of the Royal Astronomical Society, Volume 353, Issue 3, pp. 747-759

[9] T. Padmanabhan, Cosmological constant - the weight of the vacuum, Phys. Rept. 380, 2003, 235.

[10] P. J. Peebles, B. Ratra, The cosmological constant and dark energy, Rev. Mod. Phys, 75, 2003, 559, 22, 2003

[11] International Journal of Modern Physics DVol. 17, No. 11, pp. 2007-2015 (2008) Regular PapersNo Access

[12] Sanil Unnikrishnan and T. R. Seshadri, Cosmic Acceleration in A Model of Scalar-Tensor Gravitation, https://doi.org/10.1142/S0218271808013674Cited by:2

[13] Feng C J, Li X Z, Sa Ridakis E N. Preventing eternality in phantom inflation [J]. Phys Rev D, 2010, 82(2) : 023526.

[14] Cai Y F, Wang Y. Noncommutative eternal inflation possible [J]. Journal of Cosmology and Astroparticle Physics, 2007, 06: 022, 1 - 10.

[15] Donatello Dolce. Compact Time and Determinism for Bosons: Foundations. Foundations of Physics. 2010, 41 (2): 178-203.

[16] S. L. Dubovsky, S. M. Sibiryakov, Spontaneous breaking of Lorentz invariance, black holes and perpetuum mobile of the 2nd kind, Phys.Lett. B638, 509-514, 2006.

[17] Alday, Luis; Gaiotto, Davide; Tachikawa, Yuji, Liouville correlation functions from four-dimensional gauge theories. Letters in Mathematical Physics 91 (2), 2010: 167-197.

[18] Gaiotto, Davide; Moore, Gregory; Neitzke, Andrew. Wall-crossing, Hitchin systems, and the WKB approximation. Advances in Mathematics 2341, $2013: 239-403$.

[19] Dolce, D. Gauge Interaction as Periodicity Modulation, Annals of Physics, Vol. 327, Issue 6, June 2012, pp. $1562-1592$

[20] Donatello Dolce. On the intrinsically cyclic nature of space-time in elementary particles. J. Phys. Conf. Ser. 2012, 343: 012031 .

[21] Dolce, D. Classical geometry to quantum behavior correspondence in a Virtual Extra Dimension, Annals of Physics, Vol. 327, Issue 9, September 2012, pp 2354-2387

[22] Ruth Britto, Freddy Cachazo, Edward Witten, Direct Proof Of Tree-Level Recursion Relation In Yang-Mills Theory, Phys. Rev. Lett. 2005, 94, 181602.

[23] Ernst Binz, Maurice A. de Gosson, Basil J. Hiley: Clifford Algebras in Symplectic Geometry and Quantum Mechanics, Foundations of Physics, February 2012

[24] C. Schmidhuber, Brane Supersymmetry Breaking and the Cosmological Constant: Open Problems, Nucl. Phys. B619, 603, 2001.

[25] Guth A H. The inflationary universe: A possible solution to the horizon and flatness problems [J]. Phys Rev D, 1981, 23( 347) : 347 - 356.

[26] Linde A D. A new inflationary universe scenario: A possible solution of the horizon, flatness, homogeneity, isotropy and primordial monopole problems [J]. Phys Lett B, 1982, 108( 6) : 389 - 393. 
[27] Kazunori Kohri, Takahiro Terada, Primordial black hole dark matter and LIGO/Virgo merger rate from inflation with running spectral indices: formation in the matter- and/or radiation-dominated universe, Classical and Quantum Gravity, 2018, 11.15

[28] Cláudio Nassif Cruz; Fernando Antônio da Silva, Variation of the speed of light and a minimum speed in the scenario of an inflationary universe with accelerated expansion, Physics of the Dark Universe, 2018, 10. 16

[29] Planck Collaboration. Planck 2015 results [J]. XIII. Cosmological parameters, Astronomy and Astrophysics, 2016, 13:594.

[30] Yue-Yao Xu, Xin Zhang. Comparison of dark energy models after Planck 2015 [J]. European Physical Journal, 2016, 76:588.

[31] G. Alagar Ramanujam; Keith Fitzcharles; S. Muralidharan, Physics behind the Dark Matter, Dark Energy and the inflationary expansion of the universe, Indian Journal of Physics, 2019, 6. 24

[32] Leonid Marochnik, Dark energy and inflation in a gravitational wave dominated universe, Gravitation and Cosmology, 2016, 1. 15

[33] Huang Q G, Li M. CMB power spectrum from noncommutative space-time [J]. Journal of High Energy Physics, 2003 ( 6) , 014, 1 - 6.

[34] Tsujikawa S, Maa R Tens R, B R Andenbe R Ge R R. Noncommutative inflation and the CMB [J]. Phys Lett B, 2003, 574( 3 - 4) : 141 - 148 .

[35] Silver R Stein E, Tong D. Scalar speed limits and cosmology: Acceleration from D-cceleration [J]. Phys Rev D, 2004, 70 ( 10) : 103505.

[36] Cheng C, Huang Q G. Constraints on the cosmological parameters from BICEP2, Planck and WMAP[J /OL]. arXiv: 1403.7173 [astro - ph. CO].

[37] Linde A D. Chaotic inflation [J]. Phys Lett B, 1983,129, 3 -4: 177 - 181.

[38] Armendariz-Picon, C.; Mukhanov, V.; Steinhardt, Paul J., Dynamical Solution to the Problem of a Small Cosmological Constant and Late-Time Cosmic Acceleration, Physical Review Letters, Volume 85, Issue 21, November 20, 2000, pp.4438-4441 (PhRvL Homepage)

[39] J. G. Hao, X. Z. Li, Phantom-like GCG and the Constraints of Its Parameters via Cosmological Dynamics, Phys. Rev. D68, 2003, 043501

[40] M. C. Bento, O. Bertolami, A. A. Sen, Generalized Chaplygin gas and cosmic microwave background radiation constraints, Phys. Rev. D67, 2003, 063003

[41] R. R. Caldwell, M. Kaminonkowshi, N. N. Winberg, Phys. Rev. Lett, 91, 2003, 071301

[42] A R Menda, R Iz-Picon C, Damou R T, Mukhanov F. K-inflation [J]. Phys Lett B, 1999, 458( 2-3): 209-218.

[43] Gar R Iga J, Mukhanov V F. Perturbations in K-inflation [J]. Phys Lett B 1999, 458( 2 - 3) : 219 - 225.

[44] Sen A. Rolling tachyon [J]. Journal of High Energy Physics, 2002( 04) , 048, 1 - 17.

[45] Sen A. Tachyon matter [J]. Journal of High Energy Physics, 2002( 07) , 065, 1 - 17.

[46] Alishahiha M, Silve R Stein E, Tong D. DBI in the sky [J]. Phys Rev D, 2004, 70( 12) : 123505.

[47] Li M, Yoneya T. D, particle dynamics and the space-time uncertainty relation [J]. Phys Rev Lett, 1997, 78 (7) : 1219 - 1222.

[48] Yoneya T. String theory and space-time uncertainty principle [J]. Prog Theor Phys, 2000, 103( 6): $1081-1125$

[49] B R Andenbe R Ge R R , Ho P M. Noncommutative space-time, stringy space-time uncertainty principle, and density fluctuations [J]. Phys Rev D, 2002, 66( 2) : 023517.

[50] A. N. Gorban, I. Karlin, Hilbert's 6th Problem: exact and approximate hydrodynamic manifolds for kinetic 
equations, Bull. Amer. Math. Soc., 51, 2014, no. 2, 186-246

[51] Olga Razina, Pyotr Tsyba, Bekdaulet Meirbekov and Ratbay Myrzakulov, Cosmological Einstein-Maxwell model with $g$-essence, International Journal of Modern Physics D, Volume 28, Issue 10, July 2019.

[52] G. Oliveira-Neto, L. G. Martins, G. A. Monerat and E. V. Corrêa Silva, Quantum cosmology of a HořavaLifshitz model coupled to radiation, International Journal of Modern Physics D, Volume 28, Issue 10, July 2019.

[53] Sujoy K. Modak, Cosmological particle creation beyond de Sitter, International Journal of Modern Physics D, Volume 28, Issue 9, July 2019.

[54] Beasley, Chris; Heckman, Jonathan J; Vafa, Cumrun. GUTs and exceptional branes in F-theory, I., Journal of High Energy Physics. 2009, 2009: 058.

[55] Duff, M. J. String and M-Theory: Answering the Critics. Foundations of Physics. 2011, 43: 182.

[56] F. Finster, A formulation of quantum field theory realizing a sea of interacting Dirac particles, Lett. Math. Phys. 97 (2011), no. 2, 165-183.

[57] F. Finster, Perturbative quantum field theory in the framework of the fermionic projector, J. Math. Phys. 55 (2014), no. 4, 042301.

[58] Carlo Rovelli and Francesca Vidotto, Covariant Loop Quantum Gravity, Cambridge, 2014

[59] Ashtekar, A. Loop Quantum Gravity: Four Recent Advances and a Dozen Frequently Asked Questions. 11th Marcel Grossmann Meeting on Recent Developments in Theoretical and Experimental General Relativity. 2007: 126.

[60] Ashtekar, Abhay; Lewandowski, Jerzy, Background Independent Quantum Gravity: A Status Report. Classical and Quantum Gravity 21 (15), 2004: R53-R152.

[61] Thiemann, Thomas, Lectures on Loop Quantum Gravity. Lectures Notes in Physics. Lecture Notes in Physics 631, 2003: 41-135.

[62] Sabharwal, S., Khanna, G.: Numerical solutions to lattice-refined models in loop quantum cosmology. Class. Quantum Grav. 25, 085009

[63] Bojowald, M.: The semiclassical limit of loop quantum cosmology. Class. Quantum Grav. 18, L109-L116

[64] Bojowald, M.: Homogeneous loop quantum cosmology. Class. Quantum Grav. 20, 2595-2615

[65] Kleban, Matthew; Levi, Thomas S.; Sigurdson, Kris, Observing the multiverse with cosmic wakes. Physical Review D 87 (4), 2013.

[66] Aharony, Ofer; Bergman, Oren; Jafferis, Daniel Louis; Maldacena, Juan, N = 6 superconformal ChernSimons-matter theories, M2-branes and their gravity duals. Journal of High Energy Physics 2008 (10): 091.

[67] Merali, Zeeya. Collaborative physics: string theory finds a bench mate. Nature, 2011, 478 (7369): 302-304.

[68] Rovelli, Carlo. "A Dialog on Quantum Gravity". International Journal of Modern Physics D (Gravitation; Astrophysics and Cosmology), 2003, 12 (9): 1509.

[69] L. Randall and R. Sundrum, A large mass hierarchy from a small extra dimension, Phys. Rev. Lett. 83 (1999) 3370 .

[70] L. Randall and R. Sundrum, An alternative to compactification, Phys. Rev. Lett. 83 (1999) 4690.

[71] Lepe, Samuel; Peña, Francisco; Saavedra, Joel, Randall Sundrum model with $\lambda<0$ and bulk brane viscosity, Physics Letters B, Volume 662, Issue 2, p. 217-219. 04, 2008

[72] K. Agashe, H. Davoudiasl, G. Perez, and A. Soni, Warped gravitons at the LHC and beyond, Phys. Rev. D 76 (2007) 036006.

[73] Shtanov, V. Sahni, Bouncing Braneworlds, Phys. Lett. B, 557, 1 (2003)

[74] Li X Z, Zhai X H. The tachyon inflationary models with exact mode functions [J]. Phys Rev D, 2003, 67( 6) : 067501. 
[75] Marco Valerio Battisti, Cosmological bounce from a deformed Heisenberg algebra, Phys. Rev. D, 79, 2009

[76] Ioannis Papadimitriou, Supersymmetry anomalies in new minimal supergravity, Journal of High Energy Physics, 2019, 9. 16

[77] Hiroyuki Abe, Shuntaro Aoki, Sosuke Imai, Interpolation of partial and full supersymmetry breakings in $\mathrm{N}=$ 2 supergravity, Nuclear Physics, Section B, 2019, 9. 3

[78] J. A. de Azcárraga, D. Gútiez, J. M. Izquierdo, Extended D = 3 Bargmann supergravity from a Lie algebra expansion, Nuclear Physics, Section B, 2019, 9. 3

[79] Xiande Fang, Da Tang, Ling Zheng, Experimental investigation of gravity and channel size effects on flow boiling heat transfer under hypergravity, Aerospace Science and Technology, 2019, 9. 24

[80] Paolo Di Vecchia, Andrés Luna, Stephen G. Naculich, A tale of two exponentiations in N = 8 supergravity, Physics Letters B, 2019, 9. 24

[81] Tony Pinhero, Supratik Pal, Realising mutated hilltop inflation in supergravity, Physics Letters B, 2019, 9. 5

[82] Kazunori Nakayama, A note on gravitational particle production in supergravity, Phy Letters B, 2019, 8.

[83] Yermek Aldabergenov, Auttakit Chatrabhuti, Sergei V. Ketov, Generalized dilatonaxion models of inflation, de Sitter vacua and spontaneous SUSY breaking in supergravity, The European Physical Journal C, 2019, 8. 26

[84] Yoshidome H, Miyazaki M, Shimizu Hm, Obstructive jaundice impairs hepatic sinusoidal endothelial cell function and renders liver susceptible to hepatic ischemia/reperfusion. Journal of hepatology, 2000, 7. 21

[85] Iulia M. Comsa, Moritz Firsching, Thomas Fischbacherm, SO (8) supergravity and the magic of machine learning, Journal of High Energy Physics, 2019, 8. 19

[86] Xinyi Li, Ziliang Zhu, Zirui Xu, A three-dimensional pore-scale lattice Boltzmann model for investigating the supergravity effects on charging process, Applied Energy, 2019, 8. 13

[87] Xiande Fang, Ling Zheng, Yan He, Experimental Study of Pool Boiling Critical Heat Flux on Thin Wires under Various Gravities, Microgravity Science and Technology, 2019, 8. 13

[88] Leonardo Castellani, Higher form gauge fields and their nonassociative symmetry algebras, Journal of High Energy Physics, Sept 2014

[89] L. Castellani, R. D, Auria and P. Fré, Supergravity and superstrings: a geometric perspective, World Scientific, Singapore (1991).

[90] L. Castellani, Group geometric methods in supergravity and superstring theories, Int. J. Mod. Phys. A 7 (1992) 1583

[91] L. Castellani and A. Perotto, Free differential algebras: their use in field theory and dual formulation, Lett. Math. Phys. 38 (1996) 321.

[92] L. Castellani, Lie derivatives along antisymmetric tensors and the M-theory superalgebra, J. Math. Phys. 3 (2011) 110504.

[93] G.'t Hooft, Dimensional Reduction in Quantum Gravity, THU-93/26, gr-qc/ 9310026

[94] L. Susskind, The World as A Hologram, Math. Phys., 1995, 36:6377-6396

[95] Yuting Wang, Lixin Xu, Current Observational Constraints to Holographic Dark Energy Model with New Infrared cut-off via Markov Chain Monte Carlo Method, Phys. Rev. D 81: 083523, 2010

[96] N. Granda, A. Oliveros, New infrared cut-off for the holographic scalar fields models of dark energy, Physics Letter B, Vol. 671, 2, 19, 2009

[97] Jia Tian, Jue Hou, Bin Chen, Holographic correlators on integrable superstrata, Nuclear Physics, Section B, 2019, 9.24

[98] Bojowald, M., Skirzewski, A.: Effective equations of motion for quantum systems. Rev. Math. Phys. 2006, 
$18,713-745$

[99] Bojowald, M., Skirzewski, A.: Quantum gravity and higher curvature actions. Int. J. Geom. Meth. Mod. Phys. $2006,4,25-52$.

[100] Singh, P.: Loop cosmological dynamics and dualities with Randall-Sundrum braneworlds. Phys. Rev. D, 2006, 73, 063508

[101] S. H. Pereira; T. M. Guimarães, From inflation to recent cosmic acceleration: the fermionic Elko field driving the evolution of the universe, Journal of Cosmology and Astroparticle Physics, 2017, 9. 1

[102] Feng C J, Li X Z, Is non-minimal inflation eternal [J]. Nucl Phys B, 2010, 841(1-2) : 178-187.

[103] A. L. Fitzpatrick, J. Kaplan, L. Randall, and L. T. Wang, Searching for the Kaluza-Klein graviton in bulk RS models, JHEP 09 (2007) 013.

[104] W. D. Goldberger and M. B. Wise, Modulus stabilization with bulk fields, Phys. Rev. Lett. 83 (1999) 4922

[105] Zhou Xiangnan, Study of Localization-on-Membrane of Elko Field and Its Quasi Bound State outside Black Hole, Doctoral Dissertation of Lanzhou University, 2015

[106] Xu Lixin, high dimensional cosmological model and dark energy, Doctoral Dissertation of Dalian University of technology, 2006

[107] Ping Yongli, Research Based on Space-Time-Mass Theory and Membrane Model, Doctoral Dissertation of Dalian University of Technology, 2008

[108] Razieh Pourhasan et al, Out of the white hole: a holographic origin for the Big Bang, Journal of Cosmology and Astroparticle Physics, 04(2014)005

[109] Mazumder, Nairwita; Chakraborty, Subenoy, Validity of the generalized second law of thermodynamics of the universe bounded by the event horizon in holographic dark energy model, General Relativity and Gravitation, Volume 42, Issue 4, pp.813-820 (GReGr Homepage), 04, 2010

[110] Zhong Z. Z, Generation of New Solutions of the Stationary Axisymmetric Einstein Equations by A Double Complex Function Method. J. Math. Phys, 1985, 26(10): 2589-2595

[111] Yaglom I. M., A Simple Non-Euclidean Geometry and Its Physical Basis, Springer-V, New York Inc., 1979

[112] Hucks J., Hyperbolic Complex Structures in Physics, J. Math. Phys, 1993, 34(12): 5988-6008

[113] Horatiu Nastase, Introduction to supergravity, Instituto de F'1sica Te'orica, UNESP, Sao Paulo 01140-070, SP, Brazil, 2011. 12

[114] Wang, Bin; Gong, Yungui; Abdalla, Elcio, Thermodynamics of an accelerated expanding universe, Physical Review D, vol. 74, Issue 8, 10, 2006, id. 083520 (PhRvD Homepage)

[115] Izquierdo, Germán; Pavón, Diego, Dark energy and the generalized second law, Physics Letters B, Volume 633, Issue 4-5, p. 420-426. 02, 2006

[116] Edmund J. Copeland, M. Sami, Shinji Tsujikawa, Dynamics of dark energy, Int. J. Mod. Phys. D15: 1753-1936, 2006

[117] G. Hao, X. Z. Li, Phantom-like GCG and the constraints of its parameters via cosmological dynamics, Phys. Lett. B606, 2005, 7

[118] Liu D J, Li X Z. Non-commutative power-law inflation: Mode equation and spectra index [J]. Phys Lett B, 2004, 600( $1-2): 1-6$.

[119] Liu D J, Li X Z. Cosmological perturbations and noncommutative tachyon inflation [J]. Phys Rev D, 2004, 70 (12): 123504.

[120] Feng C J, Li X Z, Liu D J. Note on Power-Law Inflation in Noncommutative Space-Time [J/OL]. arXiv: 1404. 0168 [astro-ph. CO]. 
[121] Ade P A R, Aikin R W, Ba R Kats D, et al. (BICEP2 Collaboration). BICEP2 I: Detection of B-mode polarization at degree angular scales[J /OL]. arXiv: 1403. 3985[astro - ph. CO].

[122] Gibbons G W. Cosmological evolution of the rolling tachyon [J]. Phys Lett B, 2002, 537( 1 - 2) : 1 - 4.

[123] Kofman L, Linde A D. Problems with tachyon inflation [J]. J of High Energy Physics, 2002( 7) , 004, 1 - 11.

[124] Li X Z, Hao J G, Liu D J. Can quintessence be the rolling tachyon [J]. Chin Phys Lett, 2002, 19(11) : 1584 1586.

[125] Li X Z, Liu D J, Hao J G. On the tachyon inflation [J]. J. Shanghai Normal Univ. ( Natural Sciences) , 2004, 33( 4): 29- 34.

[126] Cheng C, Huang Q G. Constraint on inflation model from BICEP2 and WMAP 9-year data J/OlJ. arXiv: 1404. 1230 [astro - ph. CC].

[127] Li H, Xia J Q, Zhang X. Global fitting analysis on cosmological models after BICEP2 0/OlJ . arXiv: 1404. 0238 [astro -ph. CQ].

[128] Wu F, Li Y, Lu Y, et al. Cosmological parameter fittings with the BICEP2 data Q/Ol] . arXiv:1403. 6462 [astro - ph. CO].

[129] Lizrraga J, Urrestilla J, Da Verio D, et al. Can topological defects mimic the BICEP2 B-mode signal J/O1J . arXiv: 1403.4924 [astro - ph. CQ].

[130] M Ho C M, Hsu S D. Does the BICEP2 observation of cosmological tensor modes imply an era of nearly planckian energy densities [j/OlJ . arXiv: 1404. 0745 Jiep - ph].

[131] Ade Par, Aghanim N, ARMITAGE-CAPLAN C, et al. Planck CoUaboratioril. Planck 2013 results. XVI. Cosmological parameters 0/OlJ . arXiv:1303. 5076 [astro - ph. CCO].

[132] Esraa Elkhateeb, Dissipative unified dark fluid model, International Journal of Modern Physics D, Volume 28, Issue 9, July 2019.

[133] Renata Kallosh, Andrei Linde, Evan McDonough, Marco Scalisi. dS Vacua and the Swampland, JHEP, Jan 7, 2019. 10 pp. 134

[134] Renata Kallosh, Andrei Linde, Evan McDonough, Marco Scalisi. de Sitter Vacua with a Nilpotent Superfield, Aug 28, 2018. Fortsch. Phys. 6 pp.

[135] Andrei Linde, Dong-Gang Wang, Yvette Welling, Yusuke Yamada, Ana Achúcarro. Hypernatural inflation, Mar 27, 2018. JCAP, 25 pp.

[136] Yashar Akrami, Renata Kallosh, Andrei Linde, Valeri Vardanyan. Dark energy, $\alpha$-attractors, and large-scale structure surveys, Dec 27, 2017. JCAP, 56 pp.

[137] Ana Achúcarro, Renata Kallosh, Andrei Linde, Dong-Gang Wang, Yvette Welling. Universality of multifield $\alpha$-attractors, Nov 26, 2017. JCAP, $26 \mathrm{pp}$.

[138] Andrei Linde. On the problem of initial conditions for inflation, Oct 11, 2017. Found. Phys. 15 pp.

[139] Bob Osano and Timothy Oreta, Multi-fluid theory and cosmology: A convective variational approach to interacting dark-sector, International Journal of Modern Physics D, Vol 28, 6, July 2019.

[140] Abhineet Agarwal, R. Myrzakulov, S. K. J. Pacif and M. Shahalam, Cosmic acceleration from coupling of baryonic and dark matter components: Analysis and diagnostics, International Journal of Modern Physics D, Vol 28, 6, July 2019.

[141] Albrecht, Andreas; Steinhardt, Paul, Cosmology for Grand Unified Theories with Radiatively Induced Symmetry Breaking, Physical Review Letters, 1982, 48 (17): 1220-1223

[142] Melia, Fulvio. The Cosmic Horizon: Monthly Notices of the Royal Astronomical Society, 2007, 382 (4): $1917-1921$ 
[143] Emilio Elizalde, Martiros Khurshudyan and Shin'ichi Nojiri, Cosmological singularities in interacting dark energy models with an $\omega(q)$ parametrization, International Journal of Modern Phy. D, Vol 28, 1, July 2019.

[144] Berry M. V, Balazs N. L, Evolution of semiclassical quantum states in phase space, J. Phys. A12, 625, 1989.

[145] Maurice de Gosson, Basil Hiley: Short Time Quantum Propagator and Bohmian Trajectories, Physics Letters A, Volume 377, Issue 42, 6 December 2013, Pages 3005-3008

[146] Bernstein, Jeremy, Max Born and the Quantum Theory. Am. J. Phys. 73 (11), November 2005: 999-1008. 